\title{
Development of aptamers and aptamer-based materials for agricultural applications
}

\author{
by
}

Emily Mastronardi

A thesis submitted to the Faculty of Graduate and Postdoctoral Affairs in partial fulfillment of the requirements for the degree of

Doctor of Philosophy

in

Chemistry

Carleton University

Ottawa, Ontario

(C) 2017, Emily Mastronardi 


\section{Abstract}

With an increasing global population, there is a need for more efficient fertilizers to alleviate the negative environmental impacts of fertilizer runoff. Developments in nanotechnology could lead to fertilizers that interact with plants more efficiently. Aptamers are oligonucleotide receptors that fold into unique shapes and bind target molecules with high affinity and selectivity. Aptamers are generated through the in vitro evolution process called SELEX. Aptamers have been incorporated into sensors, diagnostics, therapeutics, targeteddelivery vehicles, and responsive materials. Biomolecules called exudates that are associated with improved nutrient uptake by crops have been identified and could serve as targets for specific fertilizer delivery. This work describes the selection of DNA aptamers binding to crop exudate, L-serine, for use in a smart fertilizer system. SELEX for small molecules has some inherent challenges, and three SELEX strategies paired with high-throughput sequencing analysis were implemented to improve the selection process. Aptamers developed from an original DNA library, as well as from DNA libraries modified from a previous small molecule selection are described. Aptamers binding to L-serine in solution were identified, and were modified to lower their production cost, and to increase their stability for use in fertilizer applications. Finally, an aptamer-polymersome nanostructure was investigated for potential use as a targeted-delivery vehicle. 


\section{Acknowledgements}

This work could not have been completed without the endless support from my supervisor Dr. Maria DeRosa. Her optimism and positivity were a constant source of inspiration, and if I can take even a small amount of her enthusiasm with me, I will be a better researcher and a better person. I would like to thank all the members of the DeRosa lab (past and present) for their shared excitement when things were working, and for their commiseration when they were not. I also thank Dr. Carlos Monreal at Agriculture and Agri-Food Canada for his support on this project.

I would like to thank my husband Alex who has encouraged me through both degrees and believed in me even when I did not. I am thankful to my family, who always put up with my books at every event, and to my friends for supporting me even when I showed up late with goggle-marks. I would like to thank Erin in particular for selflessly helping me edit and format my thesis, and for bringing me chocolate at the perfect time. Finally, I thank all the members of the Chemistry Department for their support throughout my graduate degree. 


\section{List of publications resulting from this thesis}

Mastronardi, E., C. Monreal, M.C. DeRosa. "Personalized Medicine for Crops? Opportunities for the application of molecular recognition in agriculture." Journal of Agricultural and Food Chemistry, submitted July 2017.

Mastronardi, E., M.C. DeRosa. "Outlook of Aptamer-Based Smart Materials for Industrial Applications." In Industrial Applications for Intelligent Polymers and Coatings, M. Hosseini, A.S. Hamdy Makhlouf (eds), Springer, 2016, pp. 185-203.

Mastronardi, E., P.K. Tsae, X. Zhang, A. Pach, Y. Sultan, M.C. DeRosa. "Preparation and characterization of aptamer-polyelectrolyte films and microcapsules for biosensing and delivery applications." Methods, 2016, 97: 75-87.

Mastronardi, E., P. Tsae, X. Zhang, C. Monreal, M.C. DeRosa. "Strategic Role of Nanotechnology in Fertilizers: Potential and Limitations". In Nanotechnologies in Food and Agriculture, M. Rai, C. Ribeiro, L. Mattoso (eds), Springer, 2015, pp 25-67.

McKeague, M., E.M. McConnell, J. Cruz-Toledo, E.D. Bernard, A. Pach, E. Mastronardi, X. Zhang, M. Beking, T. Francis, A. Giamberardino, A. Cabecinha, A. Ruscito, R. ArandaRodriguez, M. Dumontier, M.C. DeRosa. "Analysis of In Vitro Aptamer Selection Parameters." Journal of Molecular Evolution, 2015, 81(5): 150-161.

Mastronardi, E., A. Foster, X. Zhang, M. C. DeRosa "Smart materials based on DNA aptamers: Taking aptasensing to the next level." Sensors, 2014, 14, 3156-3171. 


\section{Table of Contents}

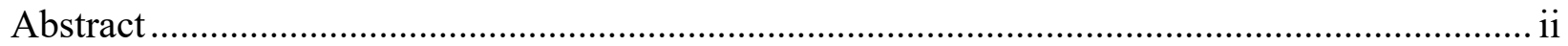

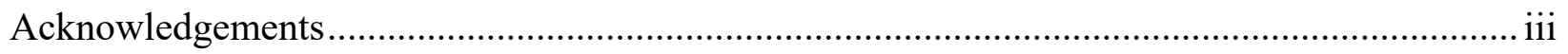

List of publications resulting from this thesis..................................................................... iv

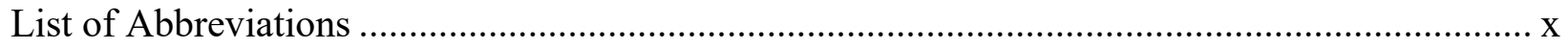

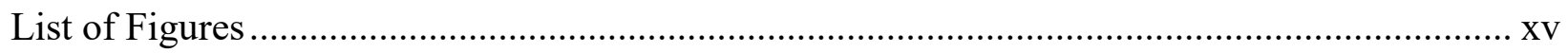

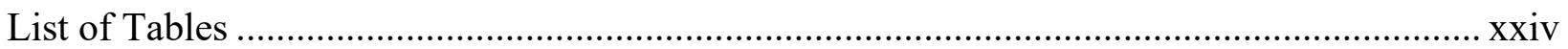

1 An introduction to aptamer smart materials and their potential application in agriculture..... 1

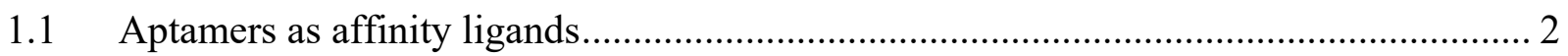

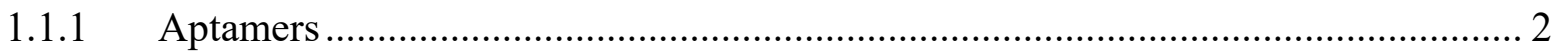

1.1.2 The systematic evolution of ligands by exponential enrichment (SELEX).............. 2

1.1.3 A comparison of aptamers to antibodies and MIPs ............................................... 5

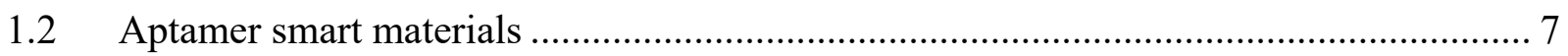

1.2.1 Incorporation of aptamers into smart materials ................................................ 7

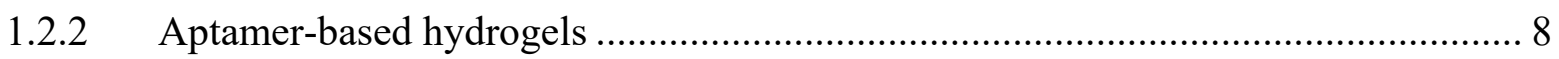

1.2.3 Aptamer-gated surfaces and nanoparticles ......................................................... 12

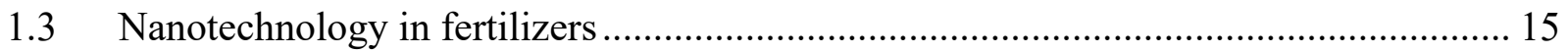

1.3.1 Challenges with current fertilizers .................................................................. 15

1.3.2 Application of nanotechnology to fertilizers ...................................................... 16

1.3.3 Nanoscale coatings and host materials ............................................................ 19

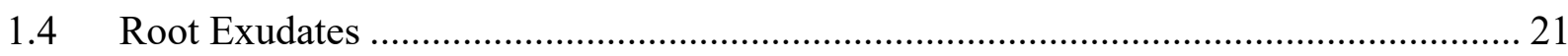

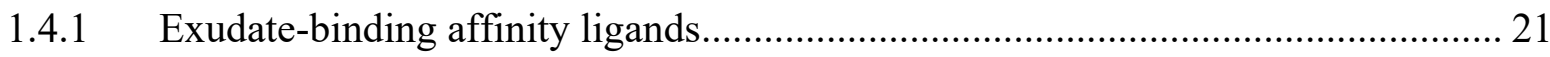

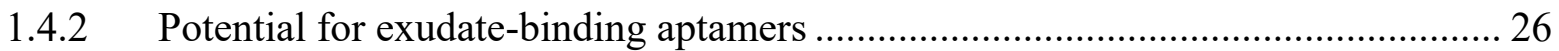

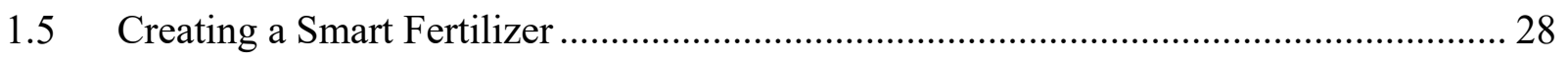

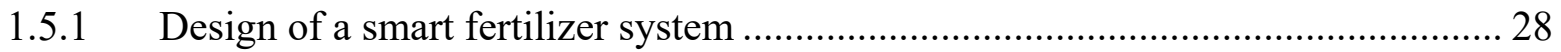

1.5.2 Progress towards smart fertilizer development................................................. 30

1.5.2.1 Identifying root exudates synchronized with nitrogen uptake ........................... 30

1.5.2.2 Confirming aptamer-target binding in thin films ............................................. 31

1.5.2.3 Creating aptamer-responsive capsules ........................................................ 36

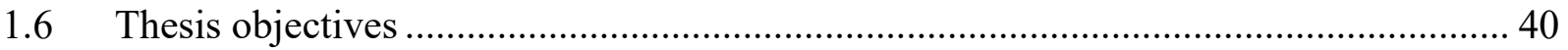


2 Selection of L-serine-binding aptamers using multiple selection strategies ..................... 42

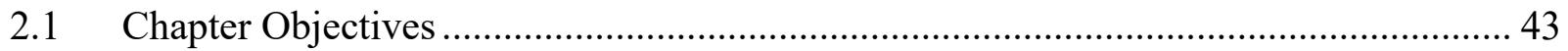

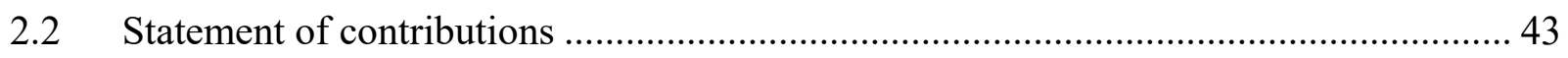

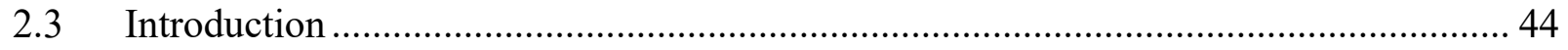

2.3.1 SELEX for small molecules.............................................................................. 44

2.3.2 Challenges in aptamer selection for small molecules ........................................ 45

2.3.3 High-throughput Sequencing (HTS) ........................................................... 47

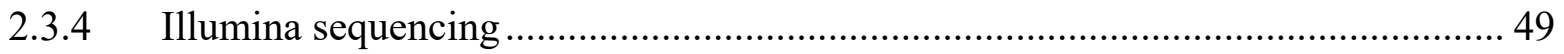

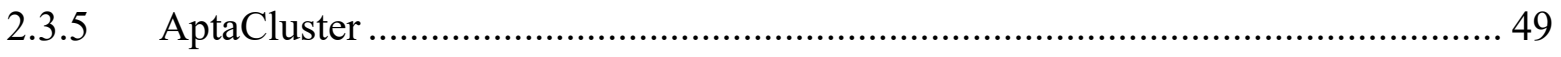

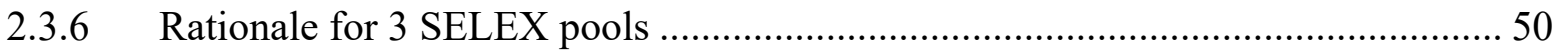

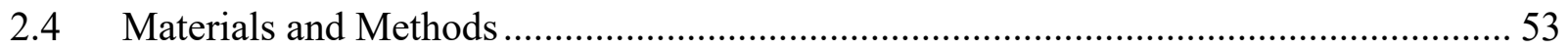

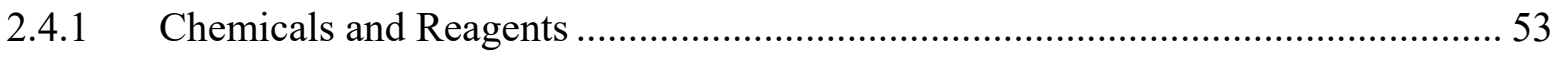

2.4.2 L-serine conjugation to sepharose beads ................................................... 54

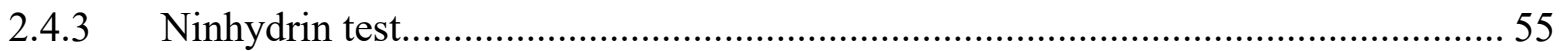

2.4.4 Testing Hcy10 affinity for L-serine ........................................................ 55

2.4.4.1 Examining Hcy10 binding to L-serine-conjugated sepharose beads ................. 55

2.4.4.2 Examining Hcy10 binding to L-serine using microscale thermophoresis ......... 56

2.4.5 DNA Synthesis of each SELEX library ............................................................ 56

2.4.6 Purification of DNA by denaturing PAGE ....................................................... 57

2.4.7 SELEX methods and Conditions for each pool .............................................. 59

2.4.8 Polymerase Chain Reaction (PCR) to amplify SELEX rounds ........................... 65

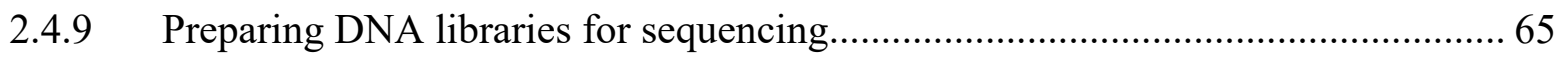

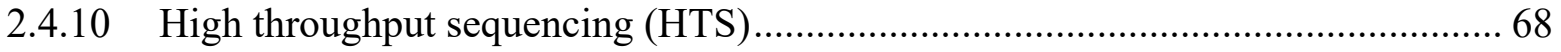

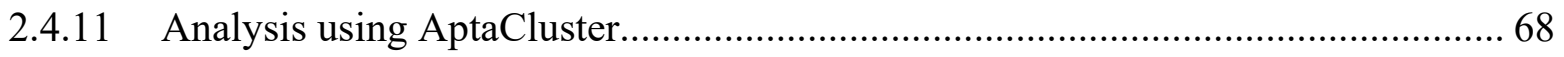

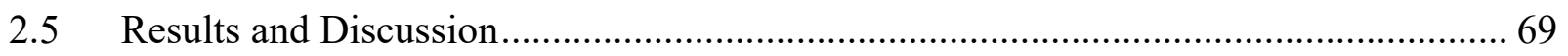

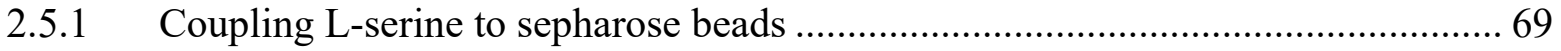

2.5.2 Evaluation of Hcy10 affinity for L-serine using multiple methods ..................... 71

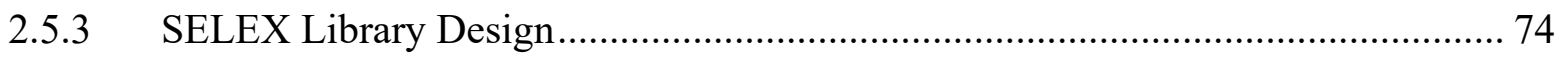

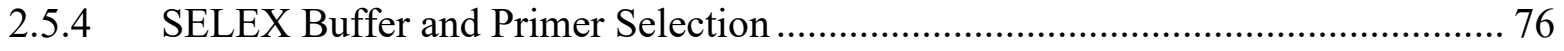

2.5.5 Monitoring SELEX Progression .................................................................... 76

2.5.6 High-throughput Sequencing (HTS).................................................... 81 


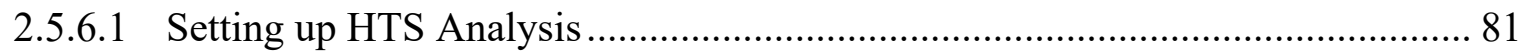

2.5.6.2 Monitoring Enrichment using AptaCluster .................................................... 83

2.5.6.3 Identifying aptamer candidates using AptaCluster ............................................. 88

2.5.6.4 Identification of Hcy10 within the Randomized and Redirected SELEX

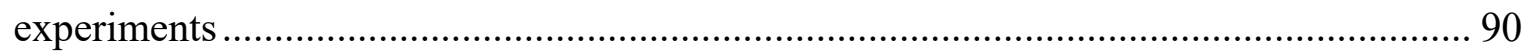

2.5.7 Analysis of Sequence Homology ……………...................................................... 93

2.5.7.1 Identification of long conserved sequence motifs ................................................ 93

2.5.7.2 Identification of short conserved sequence motifs .......................................... 99

2.5.8 Comparison of SELEX experiments.................................................................. 103

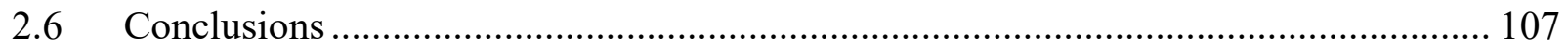

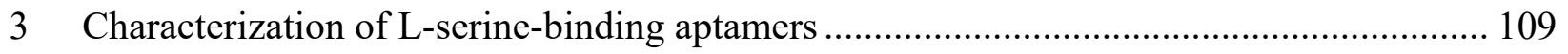

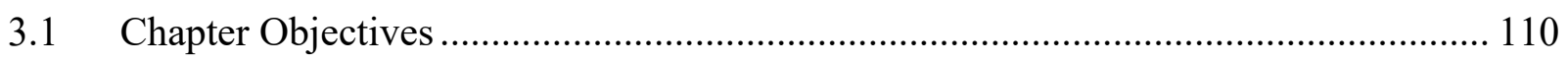

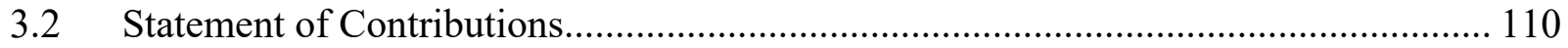

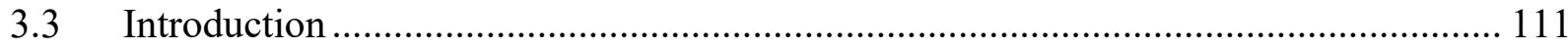

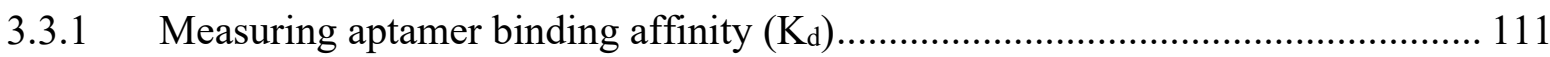

3.3.2 Challenges in measuring aptamer binding affinity $\left(\mathrm{K}_{\mathrm{d}}\right)$ for small molecule targets 112

3.3.3 Challenges with moving aptamers into application ............................................. 114

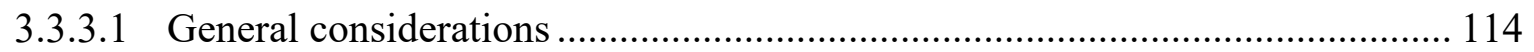

3.3.3.2 The development of minimers to increase utility of aptamers .......................... 114

3.3.3.3 Increasing the nuclease resistance of aptamers .............................................. 115

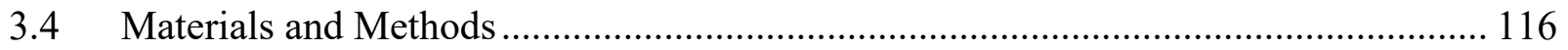

3.4.1 Chemicals and Reagents ................................................................................ 116

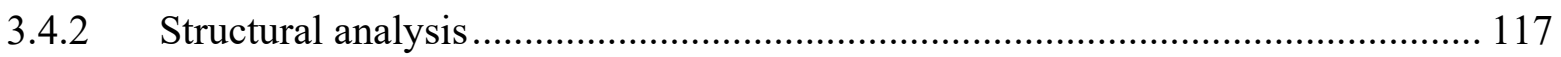

3.4.2.1 Computational Structural Analysis ............................................................ 117

3.4.2.2 Melting Temperature Analysis ....................................................................... 117

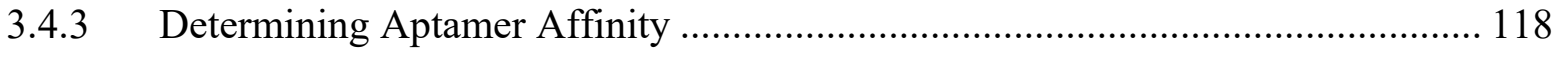

3.4.3.1 Measuring aptamer binding immobilized L-serine using L-serine-conjugated

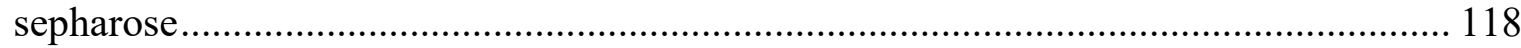

3.4.3.2 Measuring aptamer binding L-serine in solution using microscale thermophoresis 119

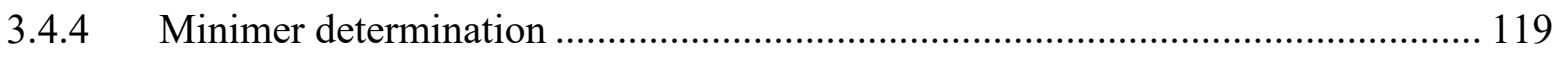


3.4.5 Examining aptamer and minimer-target binding using a colorimetric gold

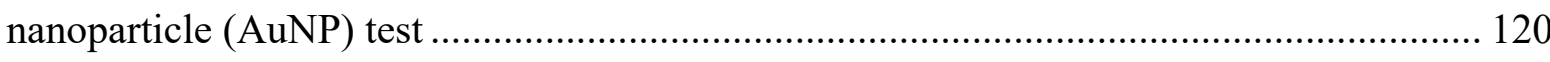

3.4.5.1 Gold nanoparticle (AuNP) synthesis......................................................... 120

3.4.5.2 Analyzing aptamer and minimer-target binding using AuNPs ........................ 120

3.4.6 Generating an L-serine-binding Spiegelmer ...................................................... 121

3.4.6.1 Spiegelmer synthesis and L-serine-binding tests ........................................... 121

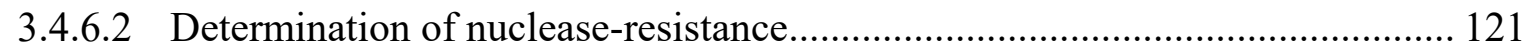

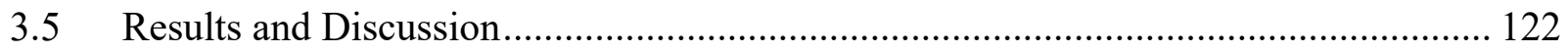

3.5.1 Binding affinity determination using L-serine-conjugated sepharose beads........ 122

3.5.2 Binding affinity determination using microscale thermophoresis (MST) ............ 127

3.5.3 Comparison of aptamer candidates generated from each SELEX strategy .......... 131

3.5.4 Structural Analysis for minimer design ........................................................... 131

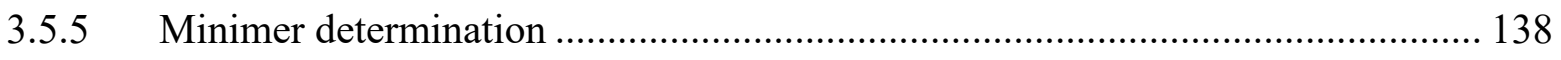

3.5.6 Colorimetric AuNP test for aptamer and minimer L-serine-binding .................... 147

3.5.7 Preparation and assessment of an L-serine-binding Spiegelmer ........................ 152

3.5.8 Examining the nuclease resistance of an L-serine-binding Spiegelmer ............... 155

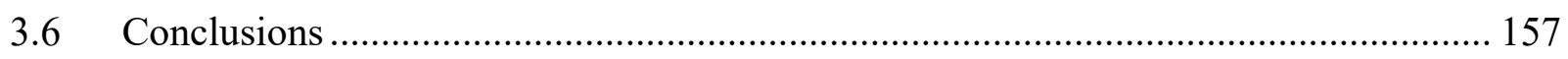

4 Synthesis and characterization of aptamer-polymersomes............................................... 158

4.1 Chapter objectives .......................................................................................... 159

4.2 Statement of contributions ………………………............................................ 159

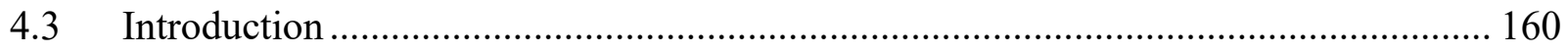

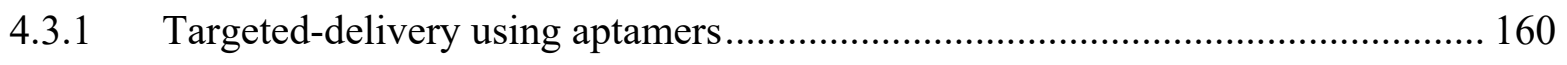

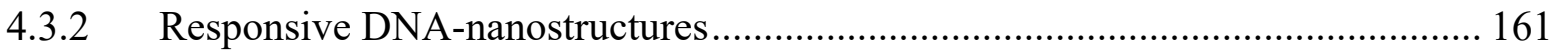

4.3.3 Incorporation of aptamers into nanostructures .................................................... 163

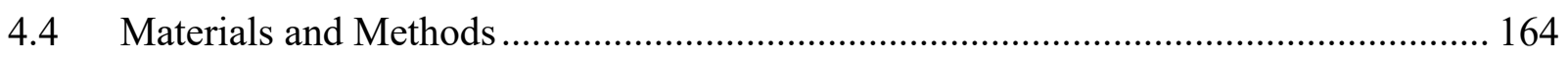

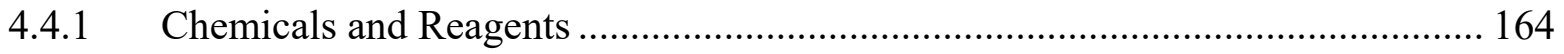

4.4.2 Synthesis of aptamer-lipid conjugates .............................................................. 165

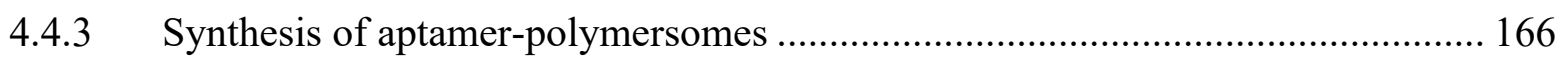

4.4.4 Imaging aptamer-polymersomes using TEM................................................... 166

4.4.5 Studying effects of carbon chain length on polymersome stability ..................... 167

4.4.6 Examining target-binding using fluorescence enhancement ................................ 167 
4.4.7 Examining target-binding using equilibrium dialysis................................... 168

4.4.8 Examining target-binding using fluorescence anisotropy................................ 168

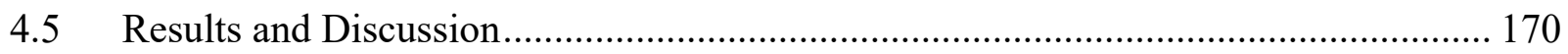

4.5.1 Design and synthesis of aptamer-lipid conjugates ......................................... 170

4.5.2 Effect of extrusion on aptamer-polymersome size ......................................... 172

4.5.3 Effect of carbon chain-length on aptamer-polymersome size ........................... 177

4.5.4 Effect of carbon-chain length on aptamer-polymersome stability ..................... 179

4.5.5 Target-binding interactions of aptamer-conjugates and aptamer-polymersomes. 184

4.5.5.1 Synthesis of random-oligonucleotide-conjugates and polymersomes ............. 184

4.5.5.2 Examining target-binding using fluorescence enhancement........................... 186

4.5.5.3 Examining target-binding using equilibrium dialysis ................................. 188

4.5.5.4 Examining target-binding using fluorescence anisotropy ............................. 189

4.5.5.5 Effect of target-binding on aptamer-polymersome morphology.................... 194

4.5.6 Conclusions and Future Directions ............................................................... 196

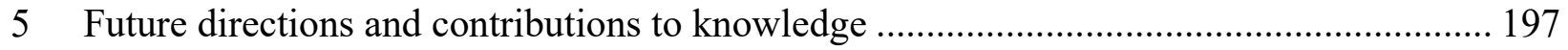

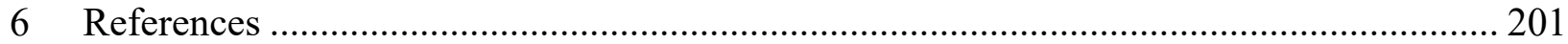

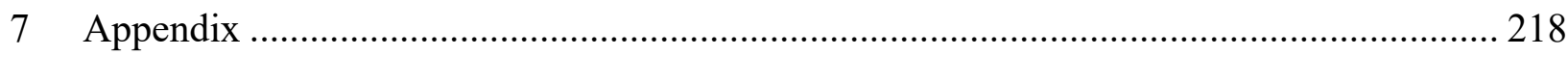




\section{List of Abbreviations}

A: adenine

AIV H5N1: avian influenza virus H5N1

ANOVA: analysis of variance

ATP: adenosine triphosphate

AuNPs: gold nanoparticles

BPA: bisphenol A

C: cytosine

CE: capillary electrophoresis

CLSM: confocal laser scanning microscopy

CPG: controlled pore glass

CT: calf thymus

CTP: cytosine triphosphate

DEA: diethylamine

$\mathrm{diH}_{2} \mathrm{O}$ : deionized water

DMF: dimethylformamide

DNA: deoxyribonucleic acid 
DNA-PE: deoxyribonucleic acid-phenyleneethynylene amphiphilic conjugate

dNTPs: deoxynucleotide triphosphates

D-VP: D-arginine vasopressin

EDTA: ethylenediaminetetraacetic acid

ESI/LC/MS: electrospray ionization liquid chromatography mass spectrometry

FACS: fluorescence activated cell-sorting

FNAs: functional nucleic acids

FRAP: fluorescence recovery after photobleaching

G: guanine

GABA: $\gamma$-aminobutyric acid

GTP: guanosine triphosphate

Hcy10: L-homocysteine aptamer 10

HPLC: high-performance liquid chromatography

HTS: high throughput sequencing

HT-SELEX: High throughput systematic evolution of ligands by exponential enrichment

$K_{d}$ : dissociation constant

LbL: Layer-by-layer 
LOD: limit of detection

LONs: lipid oligonucleotide conjugates

LSPR: localized surface plasmon resonance

MBOA: 6-methoxy-benzoxazolin-2-one

MIPs: molecularly imprinted polymers

MST: microscale thermophoresis

NHS-sepharose: N-hydroxysuccinimidyl-sepharose

OTA: ochratoxin A

PAGE: polyacrylamide gel electrophoresis

PAH: polyallylamine hydrochloride

PBS: phosphate buffered saline

PCR: polymerase chain reaction

PDDA: polydiallyldimethylammonium chloride

PDGF-BB: platelet-derived growth factor BB

PEG: polyethylene glycol

PEM: polyelectrolyte multilayer

PSS: polystyrene sulfonate 
PSMA: prostate-specific membrane antigen

QCM: quartz crystal microbalance

Ran.1: L-serine-binding aptamer candidate obtained from the Randomized SELEX in soil solution

Ran.2: L-serine-binding aptamer candidate obtained from the Randomized SELEX in buffer

Red.1: L-serine-binding aptamer candidate obtained from the Redirected SELEX in soil solution

Red.2: L-serine-binding aptamer candidate obtained from the Redirected SELEX in buffer

RNA: ribonucleic acid

RO: random oligonucleotide

SA: sulforhodamine B aptamer

SAMF: switchable aptamer micelle flare

SB: sulforhodamine B

SBS: sequencing by synthesis

SELEX: Systematic Evolution of Ligands by EXponential enrichment

Ser.1: L-serine-binding aptamer candidate obtained from the Original SELEX in soil solution

Ser.2: L-serine-binding aptamer candidate obtained from the original SELEX in buffer

SPR: surface plasmon resonance

ssDNA: single-stranded DNA 
$\mathrm{T}$ : thymine

TBE: Tris borate EDTA buffer

TEM: transmission electron microscopy

TEMED: tetramethylethylenediamine

$\mathrm{T}_{\mathrm{m}}$ : melting temperature

TMR: tetramethylrosamine

Tris: tris(hydroxymethyl) aminomethane

TTP: thymidine triphosphate

UV-Vis: ultraviolet-visible spectroscopy

VEGF: vascular endothelial growth factor 


\section{List of Figures}

Figure 1.1: Systematic Evolution of Ligands by Exponential enrichment (SELEX). A randomized DNA pool is incubated with a target of interest. Unbound sequences are partitioned from binding sequences, and binding sequences are amplified by PCR. Each cycle produces a DNA library more enriched for target-binding.

Figure 1.2: Aptamer-based hydrogel systems for controlled release. (A) A DNA linker strand (blue-red-green) causes assembly of the hydrogel through hybridization with complementary DNA strands tethered for the polymer subunits (blue and red). The linker strand contains an aptamer domain (green) which preferentially binds to the target molecule (yellow sphere), which leads to hydrogel disassembly. (B) This system can be used for the target-responsive release of a molecular payload, such as AuNPs (red spheres). (C) An aptamer-containing linker strand is used to attach a molecular payload to the hydrogel. Target-binding causes payload release, without hydrogel disassembly. Figure reproduced from Mastronardi et al. 2014 in accordance with the Sensors Creative Commons Attribution License (CC BY 3.0). ${ }^{46}$......

Figure 1.3: Aptamer and graphene oxide-gated nanochannels. Aptamers (red) were functionalized to a porous aluminum oxide membrane (dark grey). The aptamers interacted with a graphene sheet (light grey) which blocked flow (green) through the channels. In the presence of target (red sphere), aptamers let go of the graphene sheet, binding the target instead and restoring flow through the channels. Modified with permission from Zhu et al. 2015, Copyright 2014 Royal Society of Chemistry ${ }^{63}$; Reprinted from Mastronardi et al. 2016 with permission from Springer. ${ }^{9}$.....

Figure 1.4: Aptamer-gated DNA nanorobot. Hexagonal DNA "robots" were created using DNA origami with two domains held together by DNA aptamer locks. When a target (navy) was added, aptamer-target binding caused the domains to open, releasing a second target (fushia). The second target acted as the key for another robot, causing successive opening. Figure based on Amir et al. 2014 Copyright 2014 MacMillan Publishers Ltd. ${ }^{69}$ Reprinted from Mastronardi et al. 2016 with permission from Springer. ${ }^{9}$

Figure 1.5: Nanotechnology applied to fertilizer has been divided into three categories: nanoscale fertilizer inputs, nanoscale additives, and nanoscale coatings or host materials. These categories show some overlap, as some fertilizer products fall into more than one category.

Reprinted from Mastronardi et al. 2015 with permission from Springer. ${ }^{80}$. 18

Figure 1.6: Proposed designs for an aptamer smart fertilizer. A) An aptamer (green) is embedded in the walls of a polyelectrolyte (blue lines) capsule, containing a nutrient payload (blue oval). Upon recognition of an exudate (yellow), the aptamer binds the exudate, causing the 
permeability of the capsule to increase, causing a slow release of the nutrient payload. B) An aptamer (green) is embedded inside a polyelectrolyte capsule (blue and red lines), acting as a scaffold. Upon recognition of the exudate (yellow), the aptamer binds, causing the capsule to burst/collapse, and the release of the nutrient payload. Figure reproduced from Mastronardi et al. 2014 in accordance with the Sensors Creative Commons Attribution License (CC BY 3.0). ${ }^{46}$... 29

Figure 1.7: Soil components (m/z 1-3) with increased presence in the soil during periods of increased nitrogen uptake by wheat and canola. Soil components were identified using mass spectrometry, and were determined to have been exuded from the crops by using isotopically labeled $\mathrm{CO}_{2}$, and $\mathrm{N}_{2}$ in the greenhouse. Experiments were performed by the Monreal group at Agriculture and Agri-Food Canada, and the figure was provided by Dr. Carlos Monreal.......... 31

Figure 1.8: Target-binding of the sulforhodamine B aptamer embedded in PEM films. The ratio of the UV-vis absorbance of SB dye $(570 \mathrm{~nm})$ to the absorbance of DNA $(260 \mathrm{~nm})$ is shown for aptamer (SA), random oligonucleotide (RO), and calf thymus (CT) embedded PDDA/PSS PEM films. The films were exposed to $2 \mathrm{mM} \mathrm{SB}$ dye in either the absence or presence of $\mathrm{K}^{+}$, which is required for target binding. In the absence of $\mathrm{K}^{+}$, the $\mathrm{SA}$-embedded film shows no increase in target binding over the controls. In the presence of $\mathrm{K}^{+}, 2 \mathrm{x}$ better target binding is observed in the SA-embedded films. Each ratio represents the average of at least 3 measurements, and the standard deviation is shown as error bars. Figure produced with data from Sultan et al. 2009. ${ }^{244}$ Reprinted from Mastronardi et al. 2016 with permission from Elsevier. ${ }^{246}$ 33

Figure 1.9: Examination of PEM film permeability to a redox active probe. Three types of PEM films were examined in triplicate: a film containing only PAH/PSS (blue), RO-embedded PAH/PSS (red), and SA-embedded PAH/PSS (green). The diffusion coefficient for the redox active probe $\left[\mathrm{Ru}\left(\mathrm{NH}_{3}\right)_{6}\right]^{3+/ 2+}$ was measured under three conditions for each film: no target present (no dye), in the presence of a structural analog of the target (TMR), and in the presence of the aptamer's target (SB). All diffusion coefficients were normalized to the values measured under "no dye" conditions. A $2 x$ increase in diffusion coefficient was only observed when the aptamer film was exposed to its target, SB. Figure produced with data from Sultan et al. 2009. ${ }^{244}$ Reprinted from Mastronardi et al. 2016 with permission from Elsevier. ${ }^{246}$ 35

Figure 1.10: Incorporation of aptamers into PEM microcapsules. Charged polyelectrolytes (green and orange ribbons) are alternatively deposited onto a sacrificial spherical template $\left(\mathrm{CaCO}_{3}\right)$ that gets dissolved, leaving hollow microcapsules. Aptamers can be incorporated in two ways. A) After a desired number of base layers, an aptamer (yellow ribbons) can be substituted for the negatively charged polyelectrolyte for a desired number of aptamer-containing bilayers. An additional polyelectrolyte bilayer can then be added as a capping layer. In the absence of the aptamer's target, the microcapsules have limited permeability. Aptamer-target binding leads to increased permeability. B) The sacrificial spherical template $\left(\mathrm{CaCO}_{3}\right)$ has been doped with 
aptamer (yellow ribbon). After dissolution of the core, the aptamers act as a scaffold within the microcapsule. In the absence of the target, the microcapsule is stable. Aptamer-target binding causes the collapse of the microcapsule. Reprinted from Mastronardi et al. 2016 with permission from Elsevier. ${ }^{246}$

Figure 1.11: Aptamer-responsive polyelectrolyte microcapsules. A) Confocal laser scanning microscopy (CLSM) image of sulforhodamine B aptamer (SA): polystyrene sulfonate (PSS)$\mathrm{CaCO}_{3}$ microcapsules prepared with fluorescein-labeled aptamers. B) CLSM image of SA:PSS(Polydiallyldimethylammonium chloride (PDDA)/PSS)s microcapsules prepared from fluorescein-labeled aptamers. C) Colocalization experiment showing aptamer-target binding caused microcapsule rupture after 6 days. Sulforhodamine B target at $1 \mathrm{mM}$ and $0.1 \mathrm{M} \mathrm{KCl}$ was used. Inset image shows ruptured non-fluorescent aptamer microcapsule. Modified with permission from ACS Publications. ${ }^{252}$

Figure 2.1: Target types of aptamers selected between (A) 1990 and 2011, and (B) 1990 and 2013. Target-types were refined to include additional categories in (B) Figure created using data from McKeague et al. 2012, and McKeague et al. 2015. ${ }^{18,24}$

Figure 2.2: Three strategies used to generate L-serine-binding aptamers. From a previous SELEX for L-homocysteine, the Redirected SELEX, and Randomized SELEX starting libraries were generated. An Original SELEX library was also created.

Figure 2.3: Overview of SELEX experimental procedure. A DNA library was added to Trisconjugated sepharose for a "negative" selection (-). Sequences which did not bind sepharose were added to the L-serine-conjugated sepharose for a "positive" selection (+). L-serine-binding sequences were eluted with concentrated target, followed by urea.

Figure 2.4: Coupling reaction of L-serine to NHS-sepharose 4 fast flow resin 69

Figure 2.5: Colorimetric ninhydrin test showing successful coupling of L-serine to the sepharose matrix. 70

Figure 2.6: Binding isotherm showing Hcy 10 binding L-serine-conjugated sepharose beads. A range of fluorescently modified Hcy 10 concentrations $(20 \mathrm{nM}$ to $1 \mu \mathrm{M})$ was incubated with Lserine-coupled beads, and eluted with excess L-serine and $7 \mathrm{M}$ urea. Fluorescence spectroscopy was used to measure the Hcy 10 eluted. This binding isotherm was generated using simple ligand-binding in SigmaPlot, and the error is on the fit

Figure 2.7: Binding of Hcyl0 to L-serine in solution was examined using microscale thermophoresis. Fluorescently labeled-Hcy $10(20 \mathrm{nM})$ was monitored after its incubation with L- 
serine (7.6 $\mathrm{nM}-250 \mu \mathrm{M}$ in buffer). No trend was observed, indicated no binding of Hcy10 to Lserine in PBS

Figure 2.8: From the Original SELEX, the percent recovery of the ssDNA bound to the Lserine-immobilized sepharose (green) and the control Tris-blocked sepharose (red) after each selection round. In Round 10, the selective pressure was increased by reducing the amount of target, and counter selections were done against L-glycine and L-threonine prior to the positive selection. The final round was completed in soil extract.

Figure 2.9: From the Randomized SELEX, the percent recovery of the ssDNA bound to the Lserine-immobilized sepharose (green) and the control Tris-blocked sepharose (red) after each selection round. In Round 4 (data not shown), the selective pressure was increased by reducing the amount of target. Counter selections were done against L-glycine and L-threonine prior to the positive selection in Round 7. The final round was completed in soil extract.

Figure 2.10: From the Redirected SELEX, the percent recovery of the ssDNA bound to the Lserine-immobilized sepharose (green) and the control Tris-blocked sepharose (red) after each selection round. In Round 4, the selective pressure was increased by reducing the amount of target, and counter selections were done against L-glycine and L-threonine prior to the positive selection. The final round was completed in soil extract.

Figure 2.11: The pool size represented in the sequencing reaction from each SELEX round from (A) the Original SELEX; (B) the Randomized SELEX; and (C) the Redirected SELEX. 83

Figure 2.12: Sequence convergence during the Original SELEX after Illumina high throughput sequencing and analysis using AptaCluster and AptaGUI software.

Figure 2.13: Sequence convergence during the Randomized SELEX after Illumina high throughput sequencing and analysis using AptaCluster and AptaGUI software.

Figure 2.14: Sequence convergence during the Redirected SELEX after Illumina high throughput sequencing and analysis using AptaCluster and AptaGUI software.

Figure 3.1: A binding isotherm was generated from Red.1 binding L-serine-conjugated sepharose beads. A range of fluorescently modified Red.1 concentrations ( $50 \mathrm{nM}$ to $2 \mu \mathrm{M}$ ) was incubated with L-serine-coupled beads, and eluted with excess L-serine and $7 \mathrm{M}$ urea. Fluorescence spectroscopy was used to measure the Red.1 eluted. This binding isotherm was generated using simple ligand-binding in SigmaPlot, and the error is on the fit. 
Figure 3.2: Red.1 binding to L-serine-conjugated sepharose compared to a random aptamer control (81 bases). A range of fluorescently modified Red.1 and random aptamer (50 nM to 1.5 $\mu \mathrm{M}$ ) was incubated with L-serine-coupled beads, and eluted with excess L-serine and $7 \mathrm{M}$ urea. Fluorescence spectroscopy was used to measure the DNA eluted.

Figure 3.3: Aptamer candidates Ser.1, Ran.2 and Red.1 binding a range of L-serine concentrations in solution, measured by microscale thermophoresis performed by $2 \mathrm{bind}$. Fluorescently modified DNA $(20 \mathrm{nM})$ was examined after incubation with a range of L-serine concentrations $(7.6 \mathrm{nM}-250 \mu \mathrm{M})$ 128

Figure 3.4: Aptamer candidates Ser.2, Ran.1 and Red.2 showed no binding to a range of Lserine concentrations in solution, measured by microscale thermophoresis performed by $2 \mathrm{bind}$. Fluorescently modified DNA $(20 \mathrm{nM})$ was examined after incubation with a range of L-serine concentrations $(7.6 \mathrm{nM}-250 \mu \mathrm{M})$.

Figure 3.5: The secondary structure prediction for Ser.1 generated by RNAstructure software. The colour represents the probability of each base pair as a percent.

Figure 3.6: The secondary structure prediction for Ser.2 generated by RNAstructure software. The colour represents the probability of each base pair as a percent. 133

Figure 3.7: The secondary structure prediction for Ran.1 generated by RNAstructure software. The colour represents the probability of each base pair as a percent. 134

Figure 3.8: The secondary structure prediction for Ran.2 generated by RNAstructure software. The colour represents the probability of each base pair as a percent. 134

Figure 3.9: The secondary structure prediction for Red.1 generated by RNAstructure software. The colour represents the probability of each base pair as a percent. 135

Figure 3.10: The secondary structure prediction for Red.2 generated by RNAstructure software. The colour represents the probability of each base pair as a percent. 135

Figure 3.11: Thermal denaturation of Ser. 1 from $20-80^{\circ} \mathrm{C}$ examined by UV-Vis spectroscopy. SigmaPlot software was used to fit the curves using the standard curves analysis tool, and the error shown was from the fit.

Figure 3.12: Minimers were designed from full length Red.1 aptamer. (A) Red.1 structure, where the colours of each segment describe the design of minimer sequences. The green and red portions of this sequence represent the 5' and 3' primer-binding regions, respectively. (B) Each 
minimer sequence designed from the Red.1 aptamer sequence, where the colours of the sequences reflect their position in the Red.1 aptamer sequence.

Figure 3.13: Microscale thermophoresis examination of Red. 1 minimers binding L-serine in solution. All minimers $(5 \mathrm{nM})$ were $5^{\prime} \mathrm{Cy} 5$ labeled and incubated with varying concentrations of L-serine in PBS $(15 \mathrm{nM}-500 \mu \mathrm{M})$ to generate these curves, provided by 2bind, Germany. ... 142

Figure 3.14: A prediction of common secondary structures shared by the full length Red.1 aptamer and its random region minimer, generated by RNAstructure software. 143

Figure 3.15: Conserved sequence regions present in L-serine-binding aptamers (A) Ser.1, (B) Ran.2, and (C) Red.1. Conserved sequence motifs are indicated by a coloured circle (D)........ 145

Figure 3.16: Conserved sequence motifs found in the shared predicted secondary structures of Red.1 and its random region minimer. Structure prediction was done using RNAstructure software, and conserved sequence regions are indicated by coloured circles. 146

Figure 3.17: Design of an aptamer-based AuNP sensor. A) When an aptamer (black) does not bind a target (green), the aptamer is available to adsorb onto the AuNP surface (red), protecting them from salt-induced aggregation. B) When an aptamer binds a target, it no longer adsorbs to the AuNP surface, leaving the AuNPs susceptible to salt-induced aggregation (blue). 148

Figure 3.18: L-serine binding test using AuNPs with Red.1 aptamer. A) Red.1 protects AuNPs from aggregation in the presence of $0.75 \mathrm{M} \mathrm{NaCl}$. B) In the presence of $53 \mathrm{mM} \mathrm{L}$-serine, Red.1 no longer protects AuNPs, resulting in a blue colour change.

Figure 3.19: L-serine binding test using AuNPs with Random Region Minimer. A) Red.1 protects AuNPs from aggregation in the presence of $0.5 \mathrm{M} \mathrm{NaCl}$. B) In the presence of $546 \mu \mathrm{M}$ L-serine, Red.1 no longer protects AuNPs, resulting in AuNP aggregation after one week incubation.

Figure 3.20: Microscale thermophoresis analysis of Random Region Minimer and its corresponding Spiegelmer binding L and D-serine in solution. The minimer and Spiegelmer (20 $\mathrm{nM}$ ) were 5' $\mathrm{Cy} 5$ labeled and monitored after incubation with varying concentrations of L- and D-serine in PBS $(15 \mathrm{nM}-500 \mu \mathrm{M})$. The results were provided by 2 bind, Germany. 153

Figure 3.21: The stability of the L-serine-binding minimer (D-DNA) and Spiegelmer (L-DNA) in the presence of DNase I at $37^{\circ} \mathrm{C}$. After the time period indicated (time scale for the minimer is in seconds, while the time scale for the Spiegelmer is in hours), EDTA was added, DNA was heated to $90^{\circ} \mathrm{C}$ to inactivate the enzyme, and was stored at $-20^{\circ} \mathrm{C}$ until $19 \%$ denaturing PAGE 
could be performed.

Figure 4.1: Amphiphilic aptamer-lipid conjugate showing the hydrophilic sulforhodamine B aptamer head, and hydrophobic 12-carbon tail. ${ }^{311}$ 170

Figure 4.2: The synthesis of the sulforhodamine B aptamer-conjugate (expected mass $9509 \mathrm{Da}$ ) was confirmed by electrospray ionization liquid chromatography mass spectrometry (ESI/LC/MS) performed by Novatia (Newtown, USA).

Figure 4.3: Aptamer-polymersomes were formed using the thin-film hydration method. Aptamer-conjugates were dried in a glass vial. This thin film was resuspended in water, sonicated at $69^{\circ} \mathrm{C}$, and extruded through $100 \mathrm{~nm}$ pores. ${ }^{311}$ 172

Figure 4.4: The morphology of sulforhodamine B aptamer (SA)-conjugates synthesized with 12carbon chains, and extruded through a $100 \mathrm{~nm}$ filter was examined using transmission electron microscopy (TEM). (A) Aptamer-polymersomes which penetrated the $100 \mathrm{~nm}$ filter; (B). Aptamer-polymersomes which did not penetrate the $100 \mathrm{~nm}$ filter.

Figure 4.5: The size distribution of SA-polymersomes after extrusion through a filter with 100 $\mathrm{nm}$ pores was determined by measuring the TEM images using AmScope Toupview Camera software. (A) SA-polymersomes that penetrated the $100 \mathrm{~nm}$ filter; (B) SA-polymersomes that did not pass through the $100 \mathrm{~nm}$ pores. 176

Figure 4.6: The effect of the aptamer-conjugate's hydrophobic tail length on polymersome size was examined using TEM. The top row shows the aptamer-polymersome morphology with 10-, 12-, and 16-carbon hydrophobic tails, while the bottom row shows the size distribution of the corresponding aptamer-polymersomes measured using AmScope Toupview Camera software.

Figure 4.7: The stability of the aptamer-polymersomes synthesized with conjugates containing a 10-carbon hydrophobic tail was examined using TEM. The top row shows the aptamerpolymersome morphology after 1,3 , and 9 days of storage in water, while the bottom row shows the size distribution of the corresponding aptamer-polymersomes measured using AmScope Toupview Camera software. 181

Figure 4.8: The stability of the aptamer-polymersomes synthesized with conjugates containing a 12-carbon hydrophobic tail was examined using TEM. The top row shows the aptamerpolymersome morphology after 1,3 , and 9 days of storage in water, while the bottom row shows the size distribution of the corresponding aptamer-polymersomes measured using AmScope 
Figure 4.9: The stability of the aptamer-polymersomes synthesized with conjugates containing a 16-carbon hydrophobic tail was examined using TEM. The top row shows the aptamerpolymersome morphology after 1, 3, and 9 days of storage in water, while the bottom row shows the size distribution of the corresponding aptamer-polymersomes measured using AmScope Toupview Camera software.

Figure 4.10: The morphology of polymersomes synthesized using a random-oligonucleotideconjugate (RO-polymersomes) was examined using TEM (Top image). The RO-polymersomes were measured using AmScope Toupview Camera software (bottom image). 185

Figure 4.11: The interaction of the SA-conjugate with SB was examined using target-binding specific fluorescence enhancement. The fluorescence intensity of SB (100 nM) was monitored at $650 \mathrm{~nm}$ over a range of SA-conjugate concentrations $(150 \mathrm{pM}-5 \mu \mathrm{M})$.

Figure 4.12: The binding of the sulforhodamine B aptamer-conjugate (SA) and of a random oligonucleotide conjugate $(\mathrm{RO})$ to sulforhodamine $\mathrm{B}(\mathrm{SB})$ was examined using fluorescence anisotropy. The DNA-conjugates $(20 \mu \mathrm{M})$ were incubated with SB $(500 \mathrm{nM})$ for one hour prior to taking measurements. Anisotropy values were measured with excitation and emission wavelengths of $556 \mathrm{~nm}$ and $582 \mathrm{~nm}$, respectively. Error bars represent the standard deviation of six replicates. A one-way ANOVA determined a statistical difference in anisotropy values for the $\mathrm{SA}$-conjugate compared to the RO-conjugate and the SB $(\mathrm{P}<0.01)$. 190

Figure 4.13: The apparent dissociation constant of SA-conjugates binding sulforhodamine B was determined using fluorescence anisotropy. A range of SA-conjugate concentrations was incubated with $500 \mathrm{nM} \mathrm{SB}$ for one hour prior to anisotropy measurements. Anisotropy values were measured with excitation and emission wavelengths of $556 \mathrm{~nm}$ and $582 \mathrm{~nm}$, respectively. The average of six replicates was plotted using SigmaPlot software, and fit using the Standard Curves Analysis feature. An apparent $\mathrm{K}_{d}$ of $7.6 \pm 2.4 \mu \mathrm{M}$ was determined, with error from the curve fitting.

Figure 4.14: The binding of aptamer-polymersomes to sulforhodamine B was studied using fluorescence anisotropy. A range of aptamer-polymersome concentrations were incubated with $500 \mathrm{nM}$ SB for one hour. Anisotropy values were measured with excitation and emission wavelengths of $556 \mathrm{~nm}$ and $582 \mathrm{~nm}$, respectively. Error bars represent the standard deviation of six replicates. A one-way ANOVA analysis determined no statistical difference between the anisotropy values obtained for the RO-polymersomes at all concentrations. A significant difference was found for values obtained for SA-polymersomes from 15-28 $\mu \mathrm{M}$, compared to the 
highest concentration of RO-polymersomes $(\mathrm{P}<0.01)$.

Figure 4.15: The effect of target-binding on aptamer-polymersome morphology was examined using TEM (Top image). SA-polymersomes $(40 \mu \mathrm{M})$ were incubated with SB $(80 \mu \mathrm{M})$ for 30 minutes prior to preparing the sample for TEM. The SA-polymersomes were measured using AmScope Toupview Camera software (bottom image). 


\section{List of Tables}

Table 1.1: Characteristics of antibodies, aptamers, and MIPs.

Table 1.2: Affinity ligands available for select soil components identified from maize. 23

Table 1.3: Affinity ligands available for select soil components identified from wheat. 25

Table 1.4: Aptamers that have been incorporated into PEM films and microcapsules to generate smart material systems. The application and responsiveness of each system is described. Reprinted from Mastronardi et al. 2016 with permission from Elsevier. ${ }^{246}$ 34

Table 2.1: Apparent dissociation constants for DNA and RNA aptamers selected for amino acid targets.

Table 2.2: Experimental conditions in each SELEX round for the Original SELEX, Randomized SELEX, and the Redirected SELEX experiments.

Table 2.3: SELEX pools examined using Illumina high throughput sequencing Sequencing reaction 1: Randomized SELEX.

Table 2.4: Preliminary affinity screening of L-homocysteine aptamers for small molecules Lcysteine, L-methionine, and L-serine, performed by M. McKeague.

Table 2.5: The base distribution of each SELEX round throughout each SELEX experiment ... 86

Table 2.6: Count and enrichment scores of the best aptamer candidates from each selection, generated using AptaCluster after Illumina high-throughput sequencing.

Table 2.7: Aptamer candidate sequences generated from each L-serine selection experiment, after Illumina high-throughput sequencing and AptaCluster analysis.

Table 2.8: Count and Enrichment scores for the Hcyl0 aptamer found in the Randomized and Redirected selection pools, generated using AptaCluster after Illumina high-throughput sequencing.....

Table 2.9: Sequence alignment of aptamers derived from L-homocysteine selections compared to Hcy10 aptamer. 
Table 2.10: Conserved sequence motifs ( $>10$ nucleotides) in each L-serine-binding aptamer candidate and Hcy 10.

Table 2.11: Persistence of conserved sequence motifs from the starting libraries to the final rounds in each SELEX experiment.

Table 2.12: Conserved sequence motifs (4-10 nucleotides) which exclude the primer-binding regions, appearing in at least $2 \mathrm{~L}$-serine-binding aptamer candidates or Hcy 10.

Table 2.13: Conserved sequence motifs (4-10 nucleotides) which include the primer-binding regions, appearing in at least $2 \mathrm{~L}$-serine-binding aptamer candidates or Hcy10.

Table 2.14: Trends observed when comparing the Original SELEX, Randomized SELEX, and Redirected SELEX experiments. 104

Table 2.15: Trends observed in the presence of common sequence motifs in each L-serinebinding aptamer candidate.

Table 3.1: Red.1 minimer sequences ordered from IDT

Table 3.2: Apparent $K_{d}$ for aptamer candidates determined using sepharose beads conjugated to L-serine, Tris, or L-glycine as target molecules. SigmaPlot simple ligand binding was used to calculate $\mathrm{K}_{\mathrm{d} S}$, and the error represents the standard deviation between trials $(\mathrm{N}=3)$.

Table 3.3: A summary of L-serine-binding observed by each aptamer candidate using L-serineconjugated sepharose and microscale thermophoresis. 130

Table 3.4: Distribution of nucleotides in each L-serine-binding aptamer candidate. 136

Table 3.5: Melting temperatures obtained from variable-temperature UV-Vis analysis at $260 \mathrm{~nm}$, and examined using the standard curves analysis tool in SigmaPlot software. The error represents the standard deviation between three trials. 138

Table 3.6: Apparent $K_{d}$ for the RR_Minimer and Spiegelmer binding L- and D-serine, Lglycine, and Tris obtained by MST 154

Table 7.1: Primer sequences used for Illumina high-throughput sequencing 218

Table 7.2: Prevalence of DNA codons in aptamer candidate sequences. 219 
1 An introduction to aptamer smart materials and their potential application in agriculture 


\subsection{Aptamers as affinity ligands}

\subsubsection{Aptamers}

DNA and RNA are often discussed in reference to their ability to store and transfer genetic information. In addition to their biological role, oligonucleotides can be exploited for other uses including target binding and catalysis. Nucleic acids performing these roles are referred to as functional nucleic acids (FNAs) and include aptamers, ribozymes, DNAzymes, riboswitches, and oligonucleotide nanomaterials. ${ }^{1}$ Aptamers are single-stranded synthetic oligonucleotides that can fold into unique shapes, and bind target molecules with high affinity, and selectivity that has been reported to distinguish a single functional group. ${ }^{2}$ They are synthesized chemically, giving them little batch-to-batch variation as well as making them simple to modify. Aptamers have shown to have long shelf lives and low immunogenicity, making them attractive ligands for sensing and therapeutic applications. ${ }^{3}$

Aptamers are selected using an in vitro process called the systematic evolution of ligands by exponential enrichment (SELEX), and have been generated for a wide variety of targets, from small molecules, proteins, viruses, and whole cells. ${ }^{4}$ They have been incorporated into a variety of applications, including sensors, diagnostics, therapeutics, targeted delivery, and responsive materials. ${ }^{5-9}$ DNA aptamers provide greater stability than RNA aptamers, and several modifications have led to the increased nuclease-resistance, and lowered renal clearance of aptamers to be used in vivo as therapeutics. ${ }^{3,10-13}$

\subsubsection{The systematic evolution of ligands by exponential enrichment (SELEX)}

Aptamers are selected through an in vitro, iterative process called the systematic evolution of ligands by exponential enrichment (SELEX), which was first described by three separate groups in $1990 .{ }^{14-16}$ This process, described in Figure 1.1, begins with $10^{14}-10^{16}$ 
oligonucleotide sequences, each containing a large randomized region, flanked by primerbinding sites. The library is incubated with the target of interest, and an important partitioning step separates binding strands from non-binding strands. Oligonucleotide sequences that bound the target of interest can be amplified using the polymerase chain reaction (PCR), generating a new library more enriched for target-binding. Using an RNA library requires the additional steps of transcription and reverse transcription to allow for the amplification step. This process is repeated over several rounds, and the final oligonucleotide library can be sequenced, and aptamer candidates characterized and optimized for target-binding. ${ }^{17}$ 


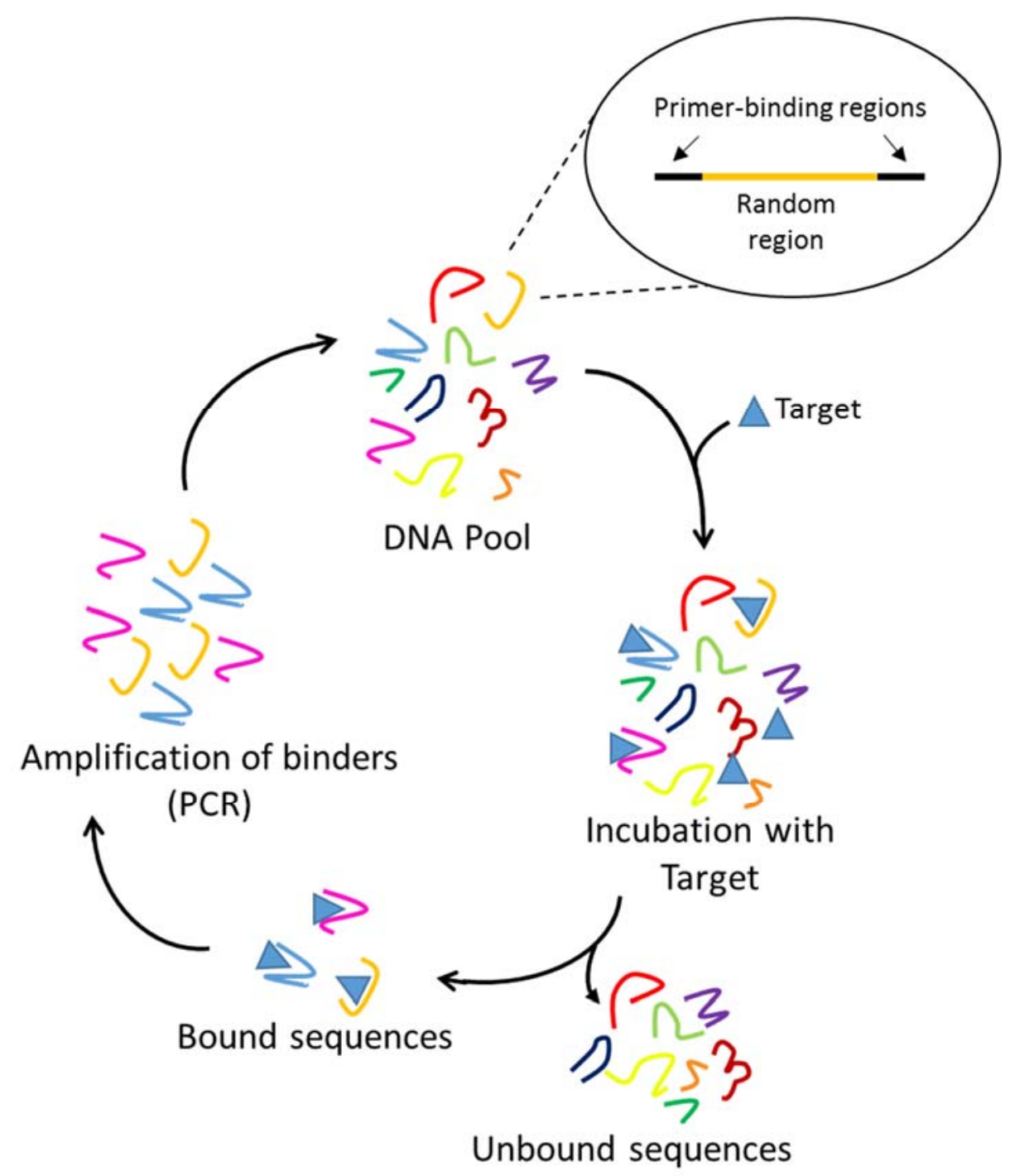

Figure 1.1: Systematic Evolution of Ligands by Exponential enrichment (SELEX). A randomized DNA pool is incubated with a target of interest. Unbound sequences are partitioned from binding sequences, and binding sequences are amplified by PCR. Each cycle produces a DNA library more enriched for target-binding.

As SELEX is an in vitro procedure, the selection conditions can be manipulated to select for aptamers capable of binding a multitude of targets in a variety of conditions. Aptamers have been selected for small molecules, toxins, carbohydrates, proteins, whole cells, and viruses. ${ }^{18}$ SELEX has also been used to identify biomarkers, when the exact target was not yet known. By counter-selecting against non-target cells, the Tan group was able to select aptamers binding unknown biomarkers on leukemia cells. ${ }^{19}$ This method has since been used extensively for 
diseased cells to generate aptamers binding many types of cancer cells, bacteria, and virusinfected cells. ${ }^{20-23}$ The selection conditions such as $\mathrm{pH}$, buffer, and temperature can all be modified to reflect downstream applications. ${ }^{18}$

Though the SELEX steps outlined in Figure 1.1 are generally followed, the SELEX procedure is amenable to modifications. Depending on the target type, the partitioning step of the SELEX process can vary. Larger targets such as cells and proteins can be separated from nonbinding oligonucleotides via filtration, while smaller targets are often immobilized to a solidsupport. Some of the first modifications to SELEX was the inclusion of "counter" selection steps, and "negative" selection steps, to eliminate sequences showing affinity to structurally similar molecules, or to the solid-support, respectively. ${ }^{24}$ Modifications have been made to the oligonucleotide library to include fixed regions of known functionality, to increase the diversity of secondary structures, or to include modified nucleotides. ${ }^{25-27}$ Improved partitioning methods, the application of more complex selection matrices, and the application of high-throughput sequencing technologies has made SELEX more efficient and more cost-effective, generating aptamers that can be directly applied in downstream applications. ${ }^{28,29}$

\subsubsection{A comparison of aptamers to antibodies and MIPs}

Affinity ligands, including aptamers, antibodies, and molecularly imprinted polymers (MIPs), can be used for many applications including detection, purification, and therapeutics. ${ }^{30}$ Antibodies have been the most widely characterized, and widely used affinity ligand due to their high affinity and specificity. ${ }^{30}$ The high cost of antibodies as well as their low stability has led to increased interest in alternative affinity ligands such as aptamers and MIPs. Molecularly imprinted polymers (MIPs) are polymers that have been synthesized in the presence of a target molecule template. After the template molecule is extracted, cavities are left in the polymer that 
are capable of binding the target. ${ }^{31}$ MIPs have some advantages over antibodies, as they are made synthetically, and have increased stability. MIPs have been generated mostly for small molecule targets, and their organic synthesis may not be amenable to biological targets. The characteristics of antibodies, aptamers, and MIPs are outlined in Table 1.1.10,12,30,31

Table 1.1: Characteristics of antibodies, aptamers, and MIPs.

\begin{tabular}{|c|c|c|c|}
\hline & Antibodies & Aptamers & MIPs \\
\hline Selection & In vivo & In vitro & In vitro \\
\hline Production & Animal or Recombinant & Synthetic & Synthetic \\
\hline $\begin{array}{c}\text { Average size } \\
\text { (kDa) }\end{array}$ & $150-160$ & $5-30$ & N/A \\
\hline Target molecules & $\begin{array}{l}\text { Immunogenic } \\
\text { macromolecules }\end{array}$ & $\begin{array}{c}\text { Small molecules, toxins, } \\
\text { macromolecules, cells, } \\
\text { viruses }\end{array}$ & Mostly small molecules \\
\hline Stability & Weeks at $4^{\circ} \mathrm{C}$ & $\begin{array}{c}\text { DNA - years at room } \\
\text { temperature } \\
\text { RNA - Months at }-80^{\circ} \mathrm{C}\end{array}$ & $\begin{array}{l}\text { Years at room } \\
\text { temperature }\end{array}$ \\
\hline $\begin{array}{l}\text { Physiological } \\
\text { half-life }\end{array}$ & Days to weeks & $\begin{array}{l}\text { Unmodified - seconds } \\
\text { to minutes } \\
\text { Modified - days }\end{array}$ & N/A \\
\hline Ease of labeling & $\begin{array}{l}\text { Feasible, heterogeneous } \\
\text { products }\end{array}$ & $\begin{array}{l}\text { Many options, } \\
\text { homogenous products }\end{array}$ & $\begin{array}{l}\text { Possible when } \\
\text { considered during } \\
\text { imprinting }\end{array}$ \\
\hline $\begin{array}{l}\text { Application } \\
\text { conditions }\end{array}$ & Physiological conditions & $\begin{array}{l}\text { Physiological, aqueous } \\
\text { non-physiological, some } \\
\text { organic solvents }\end{array}$ & $\begin{array}{l}\text { Mostly organic solvents, } \\
\text { some aqueous solutions }\end{array}$ \\
\hline
\end{tabular}

Aptamers display many advantages over antibodies and MIPs. Since they are selected using an in vitro method that does not require organic solvents, there is virtually no limitation on the potential target molecules for which aptamers can be generated. Their synthetic production method allows them to be easily modified in a homogenous way. Unlike antibodies, aptamers can be reversibly denatured, giving them a long shelf-life. Aptamers have some limitations, as they are susceptible to nuclease degradation in biological environments, and their structure is highly dependent on their surroundings. ${ }^{10,18}$ These limitations can be minimized by modifying 
the aptamers to increase their in vivo stability, and carefully planning SELEX experiments to mimic the aptamers' downstream applications.

\subsection{Aptamer smart materials}

\subsubsection{Incorporation of aptamers into smart materials}

Materials science has a growing interest in multifunctional materials. By combining engineering and physical science, new classes of responsive materials can be generated. "Smart" materials describe materials that can change their physical or chemical properties in response to an external stimulus, such as temperature, $\mathrm{pH}$, and electric or magnetic fields for example. ${ }^{32}$ Thermochromic, electrochromic, and photochromic materials have been developed, responding

to temperature, applied voltage, and light, respectively. ${ }^{33-39}$ Smart materials can be used for more than just sensing, as these materials not only sense a change in their environment, but can also adapt and report on these changes. For example, shape memory materials can alter their shape in response to stress, temperature, or changes in magnetic field, and are being studied for use as less invasive medical implants, including stents and heart valves. ${ }^{40,41}$ Smart materials can be applied to many industries, with applications in sensing, molecular electronics, and controlled release products.

Interest in using smart materials for biological applications has led to the creation of materials that are responsive to biological molecules. The incorporation of molecular recognition probes such as antibodies, MIPs, or aptamers into the material structure can make them responsive to specific biological target molecules. Aptamers are particularly well-suited to incorporation into smart material systems. ${ }^{30,42}$ Aptamers are made synthetically with a low cost of production and labeling, and little batch-to-batch variation, which facilitates their use in industrial applications. Aptamers have already been selected for a variety of biologically relevant 
targets, from small molecules to entire cells and organs. ${ }^{4-45}$ These existing aptamers could be directly applied as triggers for bioresponsive smart materials. Compared to antibodies and MIPs, DNA aptamers also offer these systems an additional level of control, as they can be inactivated by hybridization to their complement DNA strands.

Aptamers have been incorporated into smart materials, causing the properties of the materials to change as a result of aptamer-target binding. These materials do more than sense and report the target molecule. In response to the target molecule, aptamer smart materials undergo a physical change, such as the degradation of the material or the release of a molecular payload. ${ }^{46}$ Aptamers have successfully been incorporated into hydrogels, gated pores, and polyelectrolyte films. Aptamer hydrogels and gated pores are discussed in this section, while aptamer polyelectrolyte films are discussed in section 1.5.2.2.

\subsubsection{Aptamer-based hydrogels}

Hydrogel materials are composed of cross-linked hydrophilic polymers that can readily take up water, causing the material to swell. The amount of swelling can be influenced by environmental factors such as $\mathrm{pH}$, temperature, ionic strength, light, electric field, and exposure to solvents. ${ }^{47}$ Hydrogels are used in industry for the production of hygiene products, contact lenses, wound dressings, and in tissue engineering applications. ${ }^{48}$ To render a hydrogel bioresponsive, its cross-linking density must change in response to a biological stimulus. ${ }^{49}$ DNA aptamers have been incorporated into these materials, allowing the target-binding to control hydrogel swelling, or the delivery of a cargo.

The Tan group developed aptamer-based hydrogels by using DNA aptamers to cross-link polyacrylamide monomers, shown in Figure $1.2 .{ }^{49}$ Each monomer was conjugated to a short 
piece of single-stranded DNA and could be brought together through a rationally designed DNA linker strand. The linker strand contained an aptamer specific to ATP, as well as an extended region complementary to the DNA on each monomer. Adding the linker strand caused hydrogel cross-linking, while adding the target (ATP) preferentially removed the linker strand due to aptamer-target complex formation, which led to the disassembly of the hydrogel network. ${ }^{49}$ This bioresponsive hydrogel could be used for controlled delivery applications, which Tan's group demonstrated with the ATP-dependent delivery of gold nanoparticles (AuNPs) that had been sequestered within the hydrogel, seen in Figure 1.2. This method could be applied to other aptamer-target pairs, as they also saw target-derived hydrogel dissolution using the thrombin aptamer and $\alpha$-thrombin as the target molecule. Wei et al. also developed an aptamer-based hydrogel that could bind $\alpha$-thrombin, holding it within the hydrogel. ${ }^{50}$ Addition of the aptamer's complement strand led to hydrogel disassembly, and release of the $\alpha$-thrombin. 
A)

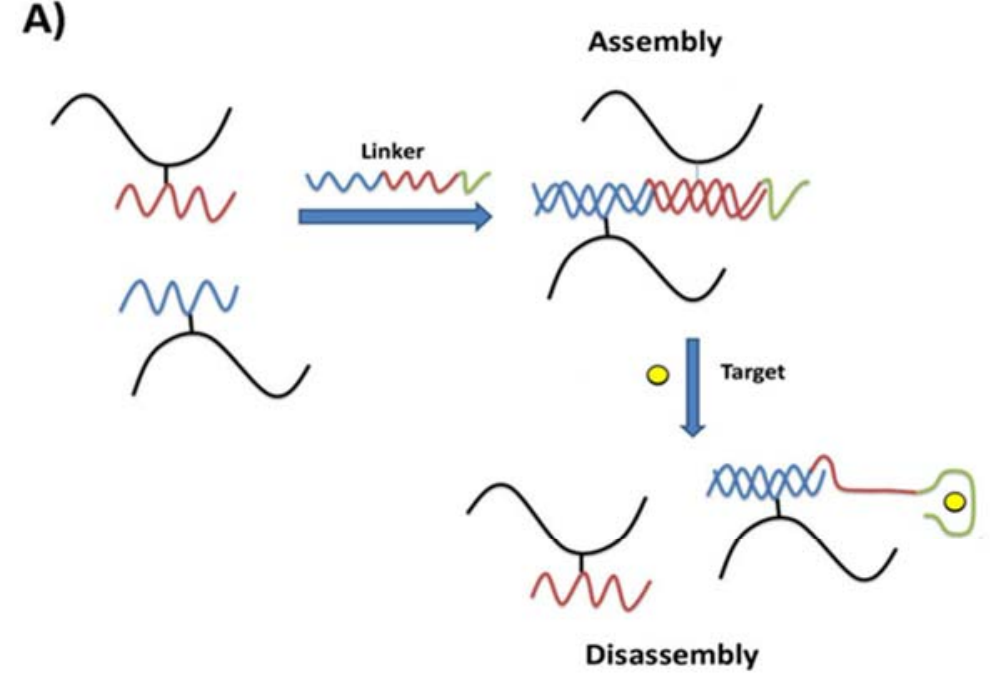

B)
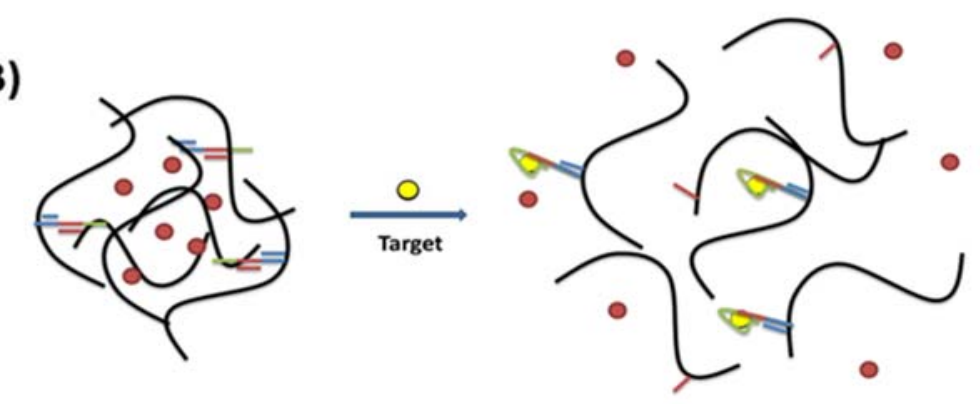

Disassembly

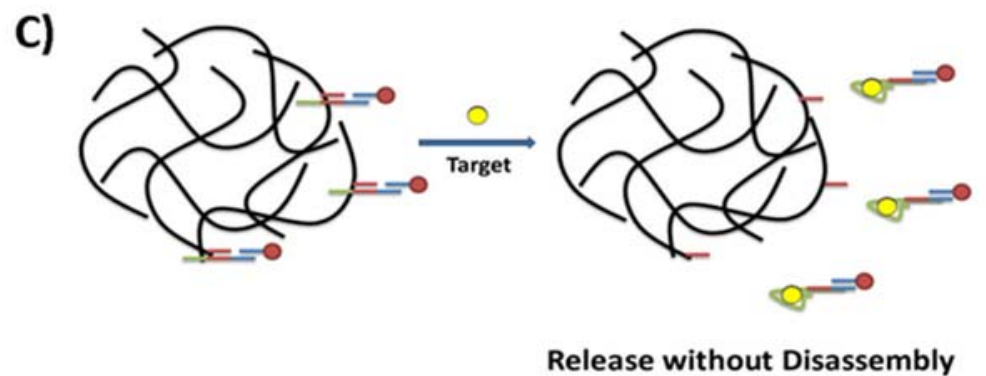

Figure 1.2: Aptamer-based hydrogel systems for controlled release. (A) A DNA linker strand (blue-red-green) causes assembly of the hydrogel through hybridization with complementary DNA strands tethered for the polymer subunits (blue and red). The linker strand contains an aptamer domain (green) which preferentially binds to the target molecule (yellow sphere), which leads to hydrogel disassembly. (B) This system can be used for the target-responsive release of a molecular payload, such as AuNPs (red spheres). (C) An aptamer-containing linker strand is used to attach a molecular payload to the hydrogel. Target-binding causes payload release, without hydrogel disassembly. Figure reproduced from Mastronardi et al. 2014 in accordance with the Sensors Creative Commons Attribution License (CC BY 3.0). ${ }^{46}$ 
Instead of using aptamers to control hydrogel cross-linking, aptamer-based hydrogels have also been developed for the controlled release of cargo. Liu and coworkers developed an aptamer-based hydrogel to create target-specific release of cargo, while the hydrogel remained intact, shown in Figure 1.2. ${ }^{51}$ Gold nanoparticles were attached to the hydrogel surface using the ATP aptamer. When ATP was introduced, the aptamer preferentially bound its target, releasing the AuNPs from the hydrogel. This cargo release was shown to be specific, not responding to structural analogs, and was able to be generalized, giving the same target-specific release of rhodamine-labeled liposomes. The Wang group developed aptamer-based hydrogels capable of releasing multiple protein targets on demand, utilizing the aptamers' predictable hybridization to their complementary strands. ${ }^{52,53}$ Aptamer-target binding was able to hold proteins VEGF and PDGF-BB within a hydrogel until the addition of each aptamer's complement strand triggered specific protein release, leaving the release of the other protein unaffected. Responsive hydrogel systems could be used for promising new drug formulations.

The creation of aptamer-based hydrogel materials undergoing target-derived physical changes or causing a payload delivery could have applications in sensing and novel drug development. Novel "catch and release" aptamer hydrogels have been created that are capable of recognizing and separating a specific target molecule from a complex mixture, which could have applications in molecular separations and environmental monitoring for example. ${ }^{54}$ Rationally designed aptamer hydrogels have also been used to perform logic operations. Aptamer-based logic gates have been described that are capable of responding to multiple targets with high specificity, using AuNPs as a visual output. ${ }^{55-61}$ These systems could be applied to make specific biosensors. 


\subsubsection{Aptamer-gated surfaces and nanoparticles}

Mimicking biological pores and channels, such as the gated ion channels found in cells, is of great interest to facilitate transport, and to create molecular actuators and transducers. ${ }^{62}$ Applying aptamer-target binding to these systems in order to control transport could create bioresponsive materials for delivery and sensing, and has been investigated by a number of groups. Aptamer-gating has been applied to control flow through channels, and for specific cargo delivery from porous nanoparticles.

Zhu et al. created gated nanochannels using a porous anodic aluminum oxide membrane containing a regular arrangement of $25 \mathrm{~nm}$ channels, gated with ATP-aptamers and graphene oxide (See Figure 1.3). ${ }^{63}$ The membrane contained a thin film of gold allowing the thiolmodified ATP aptamers to be functionalized to its surface. These single-stranded aptamers are able to interact with graphene oxide through $\pi-\pi$ stacking, keeping the rigid graphene oxide sheet close enough to block the flow through the channel. Upon introduction of ATP, the aptamer binds its target, letting go of the graphene oxide sheet and allowing the channel to open. The authors successfully impeded the flow of glucose, bovine serum albumin, and gold nanoparticles. The specificity of this responsive gate was also confirmed as structural analogs CTP, GTP, and TTP were unable to restore flow through the channels. The use of such a responsive gate can be imagined in controlled release and molecular separation applications. 

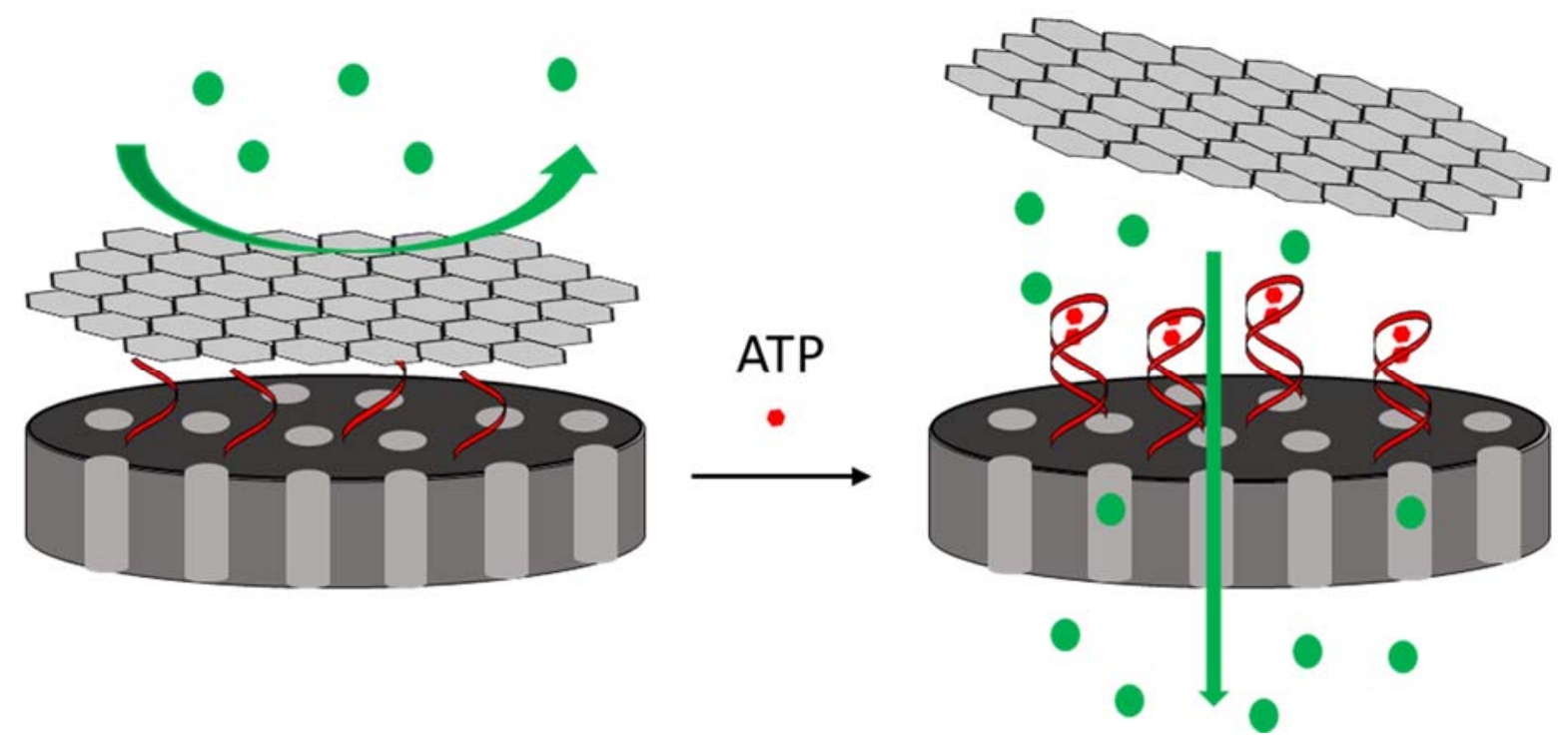

Figure 1.3: Aptamer and graphene oxide-gated nanochannels. Aptamers (red) were functionalized to a porous aluminum oxide membrane (dark grey). The aptamers interacted with a graphene sheet (light grey) which blocked flow (green) through the channels. In the presence of target (red sphere), aptamers let go of the graphene sheet, binding the target instead and restoring flow through the channels. Modified with permission from Zhu et al. 2015, Copyright 2014 Royal Society of Chemistry ${ }^{63}$; Reprinted from Mastronardi et al. 2016 with permission from Springer. ${ }^{9}$

Aptamers have been used to physically block the passage of molecules through a channel.

For example, the passage of fluorescein sodium salt through a membrane was controlled by exploiting the structural change of a modified ATP aptamer upon target binding. ${ }^{64}$ Without an ATP target, the hairpin structure allowed the passage of fluorescein sodium salt through the membrane. In the presence of ATP, the structure of the aptamer changed, and no longer allowed the fluorescein to permeate the membrane. This aptamer-gating technology was used to create a sensitive, label-free detection system for AIV H5N1 virus. ${ }^{65}$ An aptamer selected for this virus was used to block $20 \mathrm{~nm}$ pores in an enzyme-electrode detection system, by hybridizing to short complementary DNA probes on the electrode surface. In the absence of the AIV H5N1 virus, the enzyme's substrate and cofactors were blocked from reaching the enzyme-electrode. In the presence of the target virus, the aptamer preferentially bound the virus, dehybridizing from the short DNA probes and restoring the flow of substrate and cofactors to the enzyme-electrode. 
This detection system could be applied using the many aptamers selected for biomarkers, toxins, and viruses.

Porous nanoparticles can serve as delivery vehicles and can be made bioresponsive by gating these pores using DNA aptamers, resulting in target-derived payload delivery. Aptamergating has been used to mediate the release of a fluorescein payload from mesoporous silica for example. Both non-reversible and reversible delivery systems were created with these particles using the ATP aptamer as a gate. ${ }^{64,66}$ Using exclusively DNA, Douglas et al. reported and patented a DNA nanorobot controlled by an aptamer gate, which can change its shape in response to a stimulus molecule and release a payload. ${ }^{67,68}$ The hexagonal robot was created using DNA origami, having two domains held together with single-stranded DNA hinges. The robot was closed with two aptamer-complement locks, which consisted of aptamer strands on one domain hybridizing to partially complementary strands on the other domain. When specific target molecules bound both aptamers, the aptamers dehybridized from their complement strands allowing the DNA robot to open and sequestered cargo to be released. The authors demonstrated the specificity of this system with aptamer-mediated labeling of specific cell types. These nanorobots were also used to affect cell behaviour, arresting the growth of leukemic cells after aptamer-mediated release of antibodies. More recently, the authors expanded their DNA nanorobot system creating logic gates, by using the key for one robot as cargo in another, for example, such that the opening of the first robot from an external stimulus released the key for the second, allowing robots to open in succession, shown in Figure 1.4. ${ }^{69}$ This type of specific delivery could provide computational control of therapeutic delivery. 

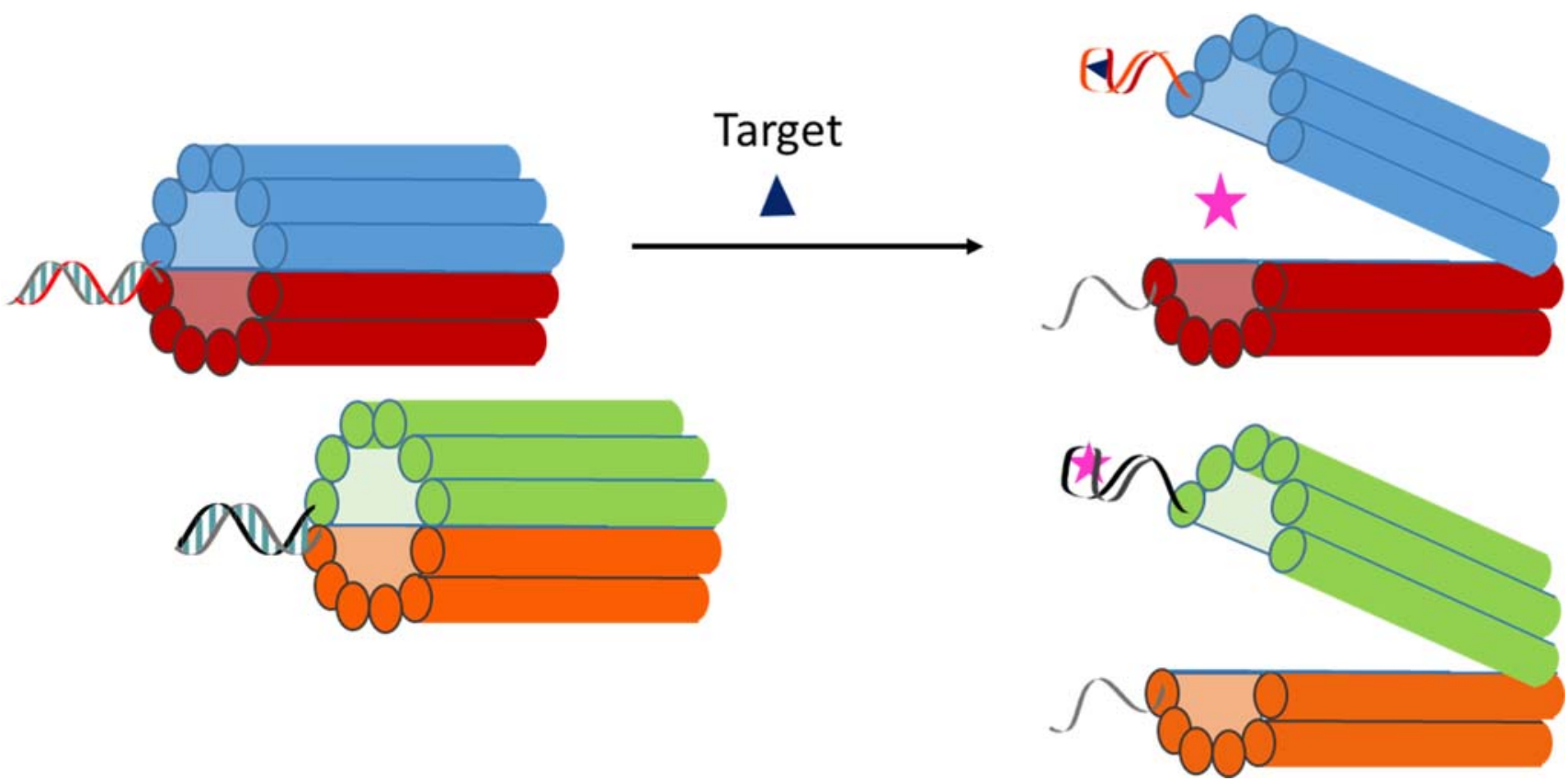

Figure 1.4: Aptamer-gated DNA nanorobot. Hexagonal DNA "robots" were created using DNA origami with two domains held together by DNA aptamer locks. When a target (navy) was added, aptamer-target binding caused the domains to open, releasing a second target (fushia). The second target acted as the key for another robot, causing successive opening. Figure based on Amir et al. 2014 Copyright 2014 MacMillan Publishers Ltd. ${ }^{69}$ Reprinted from Mastronardi et al. 2016 with permission from Springer.?

\subsection{Nanotechnology in fertilizers}

\subsubsection{Challenges with current fertilizers}

Fertilizers are natural or synthetic products that are applied to soil in order to provide all the nutrients required by crops, and are invaluable to increasing crop yields. With a growing global population, the demand for food is increasing while the available arable land is shrinking, necessitating improved efficiency in food production. The use of commercial fertilizers is essential for obtaining improved crop yields, but conventional fertilizers face efficiency issues. It has been reported that $50-70 \%$ of the nitrogen applied in conventional fertilizers does not get absorbed by the plant, but is leached into the surrounding environment as water soluble nitrates or into the atmosphere as gaseous ammonia and nitrogen oxides. ${ }^{70}$ Phosphorus use efficiency has also been reported to be very low, and this excess nitrogen and phosphorus from fertilizer 
application can cause environmental issues, such as eutrophication of aquatic ecosystems for example. In addition to environmental issues, the low efficiency of conventional fertilizers poses an economic problem as well. It has been reported that an increase in nitrogen use efficiency of only $20 \%$ could improve the annual economic performance of farmers worldwide by $\$ 4.7$ billion. ${ }^{71}$ Strategies to increase the nitrogen use efficiency of crops have included crop-rotation, genetic selection, and in-season nitrogen application, but a comprehensive solution has yet to be found. ${ }^{71}$

\subsubsection{Application of nanotechnology to fertilizers}

Nanotechnology refers to a range of technology involving matter at the length scale of 1$100 \mathrm{~nm}$. The small scale of these particles can lead to changes in their physical and chemical properties, compared to the bulk material. Nanotechnology has been applied to many fields, including healthcare, materials science, and electronics, and is also present in many consumer products such as textiles and sports equipment. Applying nanotechnology to agriculture, in the form of nanofertilizers and nanopesticides for example, has the potential to increase productivity. Nanomaterials have a smaller size and increased surface area, which could allow nanofertilizers to interact with plants more efficiently ${ }^{70}$ Incorporating nanotechnology into fertilizer products could improve the nutrient release profiles and increase the nutrient uptake efficiency, leading to significant economic and environmental benefits.

Many physical aspects of plant biology involve nanoscale structures. Plant cell walls, plant roots, and plant leaves have all been found to contain nanoscale pores. ${ }^{72-74}$ Designing fertilizers to coincide with these pore sizes could facilitate their uptake. Nanofertilizers could make use of ion channels, endocytosis, or interact with membrane transporters or root exudates to more easily be taken up by the plant. ${ }^{75}$ The effect of particle size on the penetration of water- 
suspended particles into leaves of Vicia faba was examined using $43 \mathrm{~nm}$ and $1.1 \mu \mathrm{m}$ diameter particles. ${ }^{76}$ Over a nine day period, the nanoscale particles were able to penetrate the leaf interior while the larger particles were excluded, demonstrating the preference for nanoscale particle uptake. Once nanoscale particles are taken up by a plant, their small size may facilitate cell-tocell transport via plasmodesmata. ${ }^{77}$ Plasmodesmata are nanoscale channels, $50-60 \mathrm{~nm}$ in diameter, that cross plant cell walls, allowing for cell-to-cell communication and transport. Nanofertilizers could utilize these transport channels to more effectively deliver fertilizer nutrients. Liu et al. demonstrated the ability of single-walled carbon nanotubes to not only traverse the cell wall and cell membrane of tobacco plant cells, but to facilitate the transport of cargo fluorophores or ssDNA molecules as well. ${ }^{78}$ Silica nanoparticles have also been used to successfully transport cargo molecules across plant cell walls. ${ }^{79}$ Nanofertilizers could exploit the existing nanoscale plant architecture, improving nutrient uptake and delivery. The effects of nanoparticle uptake on plant and microbe toxicity as well as their fate and transport in the environment will need to be considered. While many studies describe the positive effects of direct nanoparticle uptake, indirect effects such as DNA damage without significant cell death have been reported. ${ }^{80,81}$ The use of biodegradable polymers in nanomaterial synthesis could mitigate the risk of toxic effects. ${ }^{82}$

Nanomaterials can be created using either "bottom-up" or "top down" approaches ${ }^{83}$ The "Bottom-up" strategy involves the self-organization of smaller building blocks to generate functional nanoscale materials. The self-assembly of lipid molecules to form liposomes is one example of the "bottom-up" strategy. The "top-down" approach converts bulk materials into nanoscale materials through physical or chemical processing, such as grinding or milling. Several strategies have been implemented for the application of nanotechnology to fertilizer 
products, and these strategies have been divided into three categories: nanoscale fertilizer inputs, nanoscale additives, and nanoscale coatings or host materials. These categories are described in Figure 1.5.

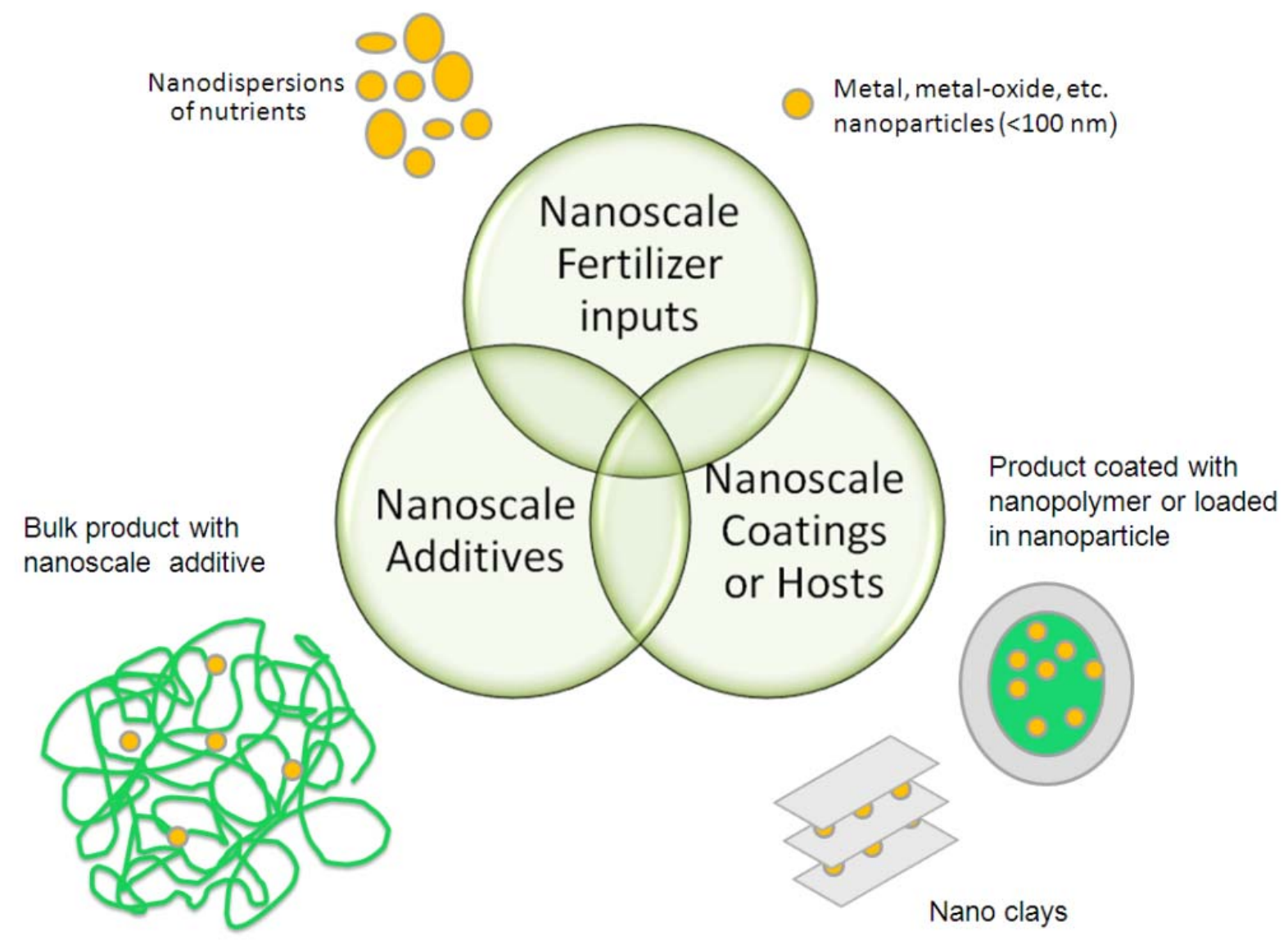

Figure 1.5: Nanotechnology applied to fertilizer has been divided into three categories: nanoscale fertilizer inputs, nanoscale additives, and nanoscale coatings or host materials. These categories show some overlap, as some fertilizer products fall into more than one category. Reprinted from Mastronardi et al. 2015 with permission from Springer. ${ }^{83}$

The category "nanoscale fertilizer inputs" describes fertilizer inputs that have been reformulated to be on the nanoscale. Mechanical or chemical methods are used to reduce the size of a fertilizer nutrient or supplement, usually resulting in nanoparticles. Zinc oxide nanoparticles applied to peanut seeds resulted in increased seed germination, greater stem and root growth, and a higher overall crop yield, for example. ${ }^{84}$ Nanoscale inputs of urea, zinc, and selenium have all 
been described ${ }^{84-89}$ "Nanoscale additives" refers to fertilizer products which have had nanomaterials added to the bulk product. These nanoscale additions could be supplement materials which were added to a fertilizer to aid in water retention, or pathogen control. The addition of carbon nanotubes has improved plant growth through increased water absorption and retention, for example. ${ }^{90-93}$ The category "nanoscale coatings or host materials" describes nanothin films or nanoporous materials, such as clays or polymer films, that are used for the controlled release of a fertilizer nutrient. Utilizing these nanoscale coatings could increase fertilizer uptake, while also protecting nutrients from leaching into the environment.

\subsubsection{Nanoscale coatings and host materials}

Creating nano-sized fertilizers to coincide with the size of plant pores could increase the uptake efficiency of these fertilizers, however their smaller size could also lead to higher dissolution rates. One strategy to combat the leaching of nutrients into the surrounding environment is to encapsulate nutrients in nanoscale films or porous host materials. Many fertilizer products have made use of clays such as kaolinites, smectites, halloysites, and palygorskites for the adsorption of nutrients. By utilizing the nanosized interlayer space within these nanoclays, fertilizers can be protected from sunlight, heat, and microbes, minimizing their loss to the environment. A montmorillonite clay was used to adsorb a magnesium-urea complex for example, and was found to protect the urea from rapid degradation. ${ }^{94}$ Zeolites, palygorskite, and kaolin clays have all been used to adsorb fertilizer nutrients, slowing their release. ${ }^{95-103}$

Fertilizer nutrients can also be encapsulated in nanotubes or nanoparticles. Halloysite nanotubes for example are hollow clay tubes, less than $100 \mathrm{~nm}$ in diameter, in which cargo can be stored for prolonged release. These nanotubes have been used for the extended release of fertilizer nutrients, as described in a patent owned by the company NaturalNano, Inc. ${ }^{104,105}$ 
Cochleate nanotubes are phospholipid-based, multi-layered structures that have also been used as delivery vehicles. ${ }^{106}$ These nanotubes are formed from a continuous lipid bilayer rolled up into a spiral, creating stable nanotubes in which cargo can be protected from the environment. Cochleate nanotubes were used to encapsulate commercial plant food, and delivered to marigolds by foliar application. When compared to the application of plant food alone, marigolds exposed to the nanotubes demonstrated larger foliage as well as more buds and blooms, which suggested improved nutrient delivery. ${ }^{107}$ Hydroxyapatite nanoparticles have been investigated for use as fertilizer carriers. ${ }^{108-113}$ Recently Kottegoda et al. incorporated urea into a matrix of hydroxyapatite nanoparticles, with a ratio of $6: 1$ by weight. ${ }^{114}$ This urea-modified hydroxyapatite nanohybrid showed a slowed release of urea over one week compared to pure urea which was expended within minutes.

Polymer films have been investigated for their ability to act as nanofertilizer coatings. Many patents have been filed for this purpose using polymer coatings made from lignosulfonate particles, polyvinyl alcohol particles, polystyrene, polyolefin-starch conjugates, cellulose, and polyelectrolytes. ${ }^{15-122}$ Polyelectrolytes polyallylamine hydrochloride (PAH) and polystyrene sulfonate (PSS) were used to create a film to encapsulate zinc sulfate. Compared to zinc sulfate alone, the polyelectrolyte-coated zinc sulfate was released into soil over a longer period, and led to increased zinc uptake by rice plants in submerged soil. ${ }^{123}$ Polymer-clay composite films have also been investigated as nanofertilizer coatings. ${ }^{124-130}$ Using polymer films to encapsulate nanofertilizers has demonstrated success in prolonging nutrient release as well as their uptake by crops. Applying aptamer technology to these films has the potential to fine-tune nutrient release even further, by requiring a specific molecular recognition event to trigger nutrient release. 


\subsection{Root Exudates}

Root exudates are compounds that get excreted by plant roots into the millimetres of soil surrounding them, called the rhizosphere. Low molecular weight compounds are the most diverse group of exudates, including organic acids, amino acids, sugars, and phenolic compounds. High molecular weight compounds such as proteins and polysaccharides make up a larger proportion of exudates by mass. ${ }^{131}$ The quantity and type of exudates released depends on the soil, plant species, and nutrient availability. ${ }^{132}$ Though the specific function of many of these exudates is unknown, many root exudates have been implicated in mediating the positive and negative interactions affecting plant and microbe growth. ${ }^{132}$ Positive interactions describe the symbiotic relationships between roots and beneficial microbes and fungi, such as mycorrhizae, rhizobia, and plant growth-promoting bacteria. ${ }^{131}$ Most terrestrial plant roots form associations with arbuscular mycorrhizal fungi, increasing their nutrient uptake while providing the fungi with lipids and carbohydrates. ${ }^{132} \mathrm{~A}$ root exudate has been implicated in this association, as a sesquiterpene known to promote hyphal branching in mycorrhizal fungi was identified in the root exudates of Lotus japonicus. ${ }^{133}$ Negative interactions refer to the interaction of roots with parasitic plants, pathogenic microbes, and invertebrate herbivores. ${ }^{131}$ Plants can protect their resources by releasing potent chemicals called phytotoxins which reduce the growth and survival of encroaching plant species. ${ }^{132}$ For example, the black walnut tree is known to produce and release the phenolic phytotoxin called juglone, making it difficult for surrounding plants to grow. ${ }^{134}$

\subsubsection{Exudate-binding affinity ligands}

Root exudates are involved in the chemical signalling that regulates many processes including microbial and fungal colonization, deterring herbivory, and inhibiting the growth of 
encroaching plant species. ${ }^{135}$ These root exudates are the means of communication between plants and the diverse array of organisms in the rhizosphere. The diversity of these compounds, their low concentrations and localized presence in soil has made their identification a challenge. With the development of sensitive mass spectrometry techniques, isotope labelling strategies, and high throughput metabolomics approaches, the identification of root exudates has become easier. $^{136,137}$

The ability to detect these exudate signals could inform on many processes. Using affinity ligands such as aptamers, antibodies, or MIPs to detect root exudates could provide information on plant health and development, or encroaching invasive species. Detecting exudates implicated in nutrient uptake could be useful in more effectively timing the application of fertilizers, while detecting specific phytotoxins could help determine appropriate pesticide application, for example. As root exudates encompass a wide range of molecules, many affinity ligands already exist for these targets and could be applied directly to agricultural applications.

Some root exudates are suspected indicators of a plant's nutrient status. Carvalhais et al. examined how the composition of root exudates was affected in maize under varying nutrient deficiencies. ${ }^{138}$ Sugars, amino acids, and organic acids exuded by maize plants were examined under iron, phosphorus, nitrogen, and potassium deficiencies. Iron deficiency stimulated increased release of glutamate, glucose, ribitol, and citrate. An examination of available affinity ligands in literature showed that an aptamer has been selected for glutamate, while MIPs for glutamate and glucose have also been developed. ${ }^{139-144}$ A phosphorus deficiency resulted in the increased release of $\gamma$-aminobutyric acid, as well as carbohydrates: inositol, erythritol, ribitol, fructose, glucose, and arabinose. Glucose and fructose-binding MIPs have been developed, while antibodies for erythritol have also been generated. ${ }^{142-146}$ A potassium deficiency resulted in the 
decreased exudation of sugars: glycerol, ribitol, fructose, and maltose, while a nitrogen deficiency showed a decrease of amino acids: aspartate, tyrosine, isoleucine, and lysine. ${ }^{138}$ Many affinity ligands have been selected for molecules identified as root exudates. These affinity ligands could be directly employed in sensors to better inform nutrient application to crops. Tables 1.2 and 1.3 show existing affinity ligands that could be used directly for sensing soil components identified from corn and wheat. Note that exudates refer to molecules released from the plant, and in the references below, the origin of these molecules was not always confirmed, and as a result are referred to as "soil components".

Table 1.2: Affinity ligands available for select soil components identified from maize.

\begin{tabular}{|c|c|c|c|c|}
\hline $\begin{array}{l}\text { Type of Soil } \\
\text { Component }\end{array}$ & $\begin{array}{l}\text { Identified Soil } \\
\text { Components }\end{array}$ & MIP & Aptamer & $\begin{array}{c}\text { Antibody } \\
\text { commercially } \\
\text { available }\end{array}$ \\
\hline \multirow[t]{12}{*}{ Amino Acids } & $\begin{array}{l}\text { Aspartic } \\
\text { Acid }^{138,147,148}\end{array}$ & $\mathrm{Yes}^{149,150}$ & No & Yes \\
\hline & $\begin{array}{l}\text { Glutamic } \\
\text { Acid }^{138,147,148}\end{array}$ & $\mathrm{Yes}^{140,141}$ & Yes $^{139}$ & Yes \\
\hline & Serine ${ }^{138,147,148}$ & Yes $^{151}$ & No & Yes \\
\hline & $\begin{array}{l}\text { Glutamine }^{138,147,1} \\
\text { (8) }\end{array}$ & Yes $^{152}$ & Yes $^{153}$ & Yes \\
\hline & Threonine ${ }^{138,148}$ & Yes $^{154}$ & No & $\begin{array}{l}\text { No, however } \\
\text { Phospho- } \\
\text { threonine } \\
\text { antibody } \\
\text { available }\end{array}$ \\
\hline & Alanine $^{138,147,148}$ & Yes $^{155}$ & No & Yes \\
\hline & Tyrosine $^{138,148}$ & Yes $^{156,157}$ & Yes $^{158}$ & $\begin{array}{l}\text { No, however } \\
\text { Phospho- } \\
\text { tyrosine } \\
\text { antibody is } \\
\text { available }\end{array}$ \\
\hline & Leucine $^{147,148}$ & No & No & Yes \\
\hline & Isoleucine $^{138,148}$ & No & Yes $^{159-161}$ & Yes \\
\hline & Glycine $^{147,148}$ & Yes $^{162,163}$ & No & Yes \\
\hline & Arginine $^{147}$ & Yes $^{164}$ & Yes $^{165-167}$ & Yes \\
\hline & Valine $^{147,148}$ & Yes $^{154}$ & Yes $^{168}$ & Yes \\
\hline
\end{tabular}




\begin{tabular}{|c|c|c|c|c|}
\hline & Histidine $^{148}$ & Yes $^{169}$ & Yes $^{170}$ & $\begin{array}{l}\text { No, however a } \\
\text { His-tag antibody } \\
\text { is available }\end{array}$ \\
\hline & Phenylalanine $^{148}$ & Yes $^{171,172}$ & Yes $^{173}$ & Yes \\
\hline & $\begin{array}{l}\gamma \text {-aminobutyrate } \\
(\text { GABA })^{138,147}\end{array}$ & $\mathrm{Yes}^{174}$ & No & Yes \\
\hline \multirow[t]{4}{*}{ Organic Acids } & $\begin{array}{l}\text { Malic } \\
\operatorname{acid}^{138,147,148}\end{array}$ & Yes $^{175}$ & No & No \\
\hline & $\begin{array}{l}\text { Succinic } \\
\operatorname{acid}^{138,147,148}\end{array}$ & No & No & Yes \\
\hline & Benzoic acid $^{147}$ & Yes $^{176}$ & No & $\begin{array}{l}\text { No, however a } \\
\text { literature } \\
\text { reference is } \\
\text { available }^{177}\end{array}$ \\
\hline & $\begin{array}{l}\text { Trans-aconitic } \\
\operatorname{acid}^{138,148}\end{array}$ & $\mathrm{Yes}^{178,179}$ & No & No \\
\hline \multirow[t]{8}{*}{ Carbohydrates } & Erythritol $^{138}$ & No & No & $\begin{array}{l}\text { No, however a } \\
\text { literature } \\
\text { reference is } \\
\text { available }^{146}\end{array}$ \\
\hline & Fructose $^{138,147,148}$ & Yes $^{145}$ & $\begin{array}{l}\text { No, however an } \\
\text { aptamer binding } \\
\text { fructose } 1,6 \\
\text { bisphosphate is } \\
\text { described. } 180\end{array}$ & No \\
\hline & Glucose $\mathrm{e}^{138,147,148}$ & Yes $^{142-144}$ & No & No \\
\hline & Sucrose $^{138,147}$ & Yes $^{181}$ & No & $\begin{array}{l}\text { No, however an } \\
\text { antibody binding } \\
\text { sucrose-6- } \\
\text { heptyl- } \\
\text { phosphonate is } \\
\text { described }^{182}\end{array}$ \\
\hline & Maltose $^{138,148}$ & Yes $^{183,184}$ & No & Yes \\
\hline & Lactose $^{148}$ & Yes $^{185}$ & No & No \\
\hline & Melibiose $^{148}$ & No & No & $\begin{array}{l}\text { No, however a } \\
\text { literature } \\
\text { reference is } \\
\text { available }^{186}\end{array}$ \\
\hline & Mannose $^{148}$ & Yes $^{187}$ & $\begin{array}{l}\text { No, however } \\
\text { aptamers } \\
\text { binding } \\
\text { mannose- } \\
\text { capped } \\
\text { lipoarabinomann } \\
\text { an are } \\
\text { described }^{188,189}\end{array}$ & No \\
\hline
\end{tabular}


Table 1.3: Affinity ligands available for select soil components identified from wheat.

\begin{tabular}{|c|c|c|c|c|}
\hline $\begin{array}{l}\text { Type of Soil } \\
\text { Component }\end{array}$ & $\begin{array}{l}\text { Identified Soil } \\
\text { Components }\end{array}$ & MIP & Aptamer & $\begin{array}{c}\text { Antibody } \\
\text { commercially } \\
\text { available }\end{array}$ \\
\hline Fatty Acid ${ }^{190}$ & Arachidonic acid & No & No & Yes \\
\hline \multirow{2}{*}{ Flavone $e^{191}$} & Flavone & Yes $^{192}$ & No & No \\
\hline & Luteolin & Yes $^{193}$ & No & No \\
\hline \multirow[t]{2}{*}{ Flavonol $^{191}$} & Kaempferide & Yes $^{194}$ & No & No \\
\hline & Quercetin & Yes $^{195}$ & No & Yes \\
\hline $\begin{array}{l}\text { Lignin } \\
\text { Monomer }{ }^{191}\end{array}$ & Ferulic acid & Yes $^{196}$ & No & Yes \\
\hline $\begin{array}{l}\text { n- } \\
\text { Alkylbenzene }{ }^{191}\end{array}$ & Methylbenzene & Yes $^{197}$ & No & No \\
\hline \multirow[t]{9}{*}{$\begin{array}{l}\text { N-Containing } \\
\text { compounds }{ }^{191}\end{array}$} & $\begin{array}{l}\text { 4- } \\
\text { Methylimidazole }\end{array}$ & Yes $^{198}$ & No & $\begin{array}{l}\text { No, however a } \\
\text { literature } \\
\text { reference is } \\
\text { available }^{199}\end{array}$ \\
\hline & Aminopyridine & Yes $^{200-202}$ & No & No \\
\hline & $\begin{array}{l}\text { Dihydroxy- } \\
\text { pyridine }\end{array}$ & No & No & Yes \\
\hline & Indole & $\mathrm{Yes}^{203}$ & $\begin{array}{l}\text { No, however an } \\
\text { aptamer for } \\
\text { tryptophan is } \\
\text { described }^{204}\end{array}$ & Yes \\
\hline & 3-Methylindole & Yes $^{205}$ & $\begin{array}{l}\text { In } \\
\text { development }{ }^{206}\end{array}$ & $\begin{array}{l}\text { No, however a } \\
\text { literature } \\
\text { reference is } \\
\text { available }^{207}\end{array}$ \\
\hline & Indolethanol & Yes $^{208}$ & No & No \\
\hline & Indole acetic acid & $\mathrm{Yes}^{209,210}$ & $\begin{array}{l}\text { No, however an } \\
\text { aptamer for the } \\
\text { hormone zeatin } \\
\text { is described }{ }^{211}\end{array}$ & Yes \\
\hline & Isoquinoline & $\begin{array}{l}\text { No, however a } \\
\text { MIP for } \\
\text { quinoline is } \\
\text { described }^{212}\end{array}$ & $\begin{array}{l}\text { No, however an } \\
\text { aptamer for } \\
\text { cocaine is } \\
\text { described }^{213}\end{array}$ & No \\
\hline & Carbazole & $\mathrm{Yes}^{214}$ & No & Yes \\
\hline Phenol ${ }^{191}$ & Phenol & Yes $^{215-219}$ & $\begin{array}{l}\text { No, however an } \\
\text { aptamer for BPA } \\
\text { is described }^{220}\end{array}$ & $\begin{array}{l}\text { No, however } \\
\text { antibodies for } \\
\text { chlorinated } \\
\text { phenols are } \\
\text { commercially } \\
\text { available }\end{array}$ \\
\hline
\end{tabular}




\begin{tabular}{|c|c|c|c|c|}
\hline & $\begin{array}{l}\text { Dihydroxy- } \\
\text { benzene }\end{array}$ & $\mathrm{Yes}^{221}$ & No & No \\
\hline & Dimethylphenol & $\mathrm{Yes}^{222}$ & No & No \\
\hline & Guaiacol & $\begin{array}{l}\text { No, however a } \\
\text { MIP for } \\
\text { chloroguaiacol is } \\
\text { described }^{223}\end{array}$ & No & No \\
\hline & $\begin{array}{l}\text { Trihydroxy- } \\
\text { benzene }\end{array}$ & $\mathrm{Yes}^{224}$ & No & No \\
\hline & Vanillin & Yes $^{225-227}$ & No & No \\
\hline & Chicoric acid & $\mathrm{Yes}^{228}$ & No & No \\
\hline \multirow[t]{5}{*}{ Sterols $^{191}$} & Cholesterol & $\mathrm{Yes}^{229,230}$ & $\begin{array}{l}\text { No, however an } \\
\text { aptamer for } 17 \beta- \\
\text { estradiol has } \\
\text { been described }^{231}\end{array}$ & Yes \\
\hline & Ergosterol & $\mathrm{Yes}^{232}$ & No & No \\
\hline & Stigmasterol & $\mathrm{Yes}^{233}$ & No & No \\
\hline & $\beta$-Sitosterol & Yes $^{234-236}$ & No & No \\
\hline & $\alpha$-Tocopherol & $Y_{e}{ }^{237-239}$ & No & Yes \\
\hline
\end{tabular}

\subsubsection{Potential for exudate-binding aptamers}

While using affinity ligands to sense the presence of particular exudates in soil could provide useful plant health information, using exudate-binding aptamers would create a unique opportunity to not only sense, but respond to soil conditions. As outlined in section 1.2, aptamers can be used in responsive materials, which could facilitate not only exudate sensing opportunities, but the development of smarter materials such as delivery systems for fertilizers and pesticides. Aptamers could sense specific exudates, and target-binding could initiate a delivery response. Glutamate was shown to be released from maize in response to iron deficiency. ${ }^{138}$ The glutamate-binding aptamer could be incorporated into a smart material encapsulating iron for example, allowing maize to receive iron on-demand. ${ }^{139}$ Exudates released as a result of nutrient deficiency would make excellent targets for aptamer development. These exudate-binding aptamers could be incorporated into smart materials encapsulating fertilizer nutrients, allowing for on-demand fertilizer release. These aptamer-mediated smart fertilizers 
could reduce the need to apply excessive fertilizer nutrients, alleviating the environmental problems associated with fertilizer over-use.

Exudate-binding aptamer smart materials could also be applied to pesticide application. Many crops release compounds which inhibit the growth of surrounding species, called allelopathy. ${ }^{132}$ L-tryptophan was identified as an allelochemical in wheat for example, and at a concentration of only $6.2 \mu \mathrm{M}$, it was able to suppress the growth of lettuce and garden cress by $47.5 \%$ and $35 \%$, respectively. ${ }^{240}$ An L-tryptophan aptamer was selected in 2011, and could be incorporated into a smart-release pesticide. ${ }^{204}$ As the aptamer detected L-tryptophan release from wheat, an additional pesticide could be specifically released to combat encroaching species. Pesticide-binding aptamers could also be employed to slow or prolong the release of specific pesticides. Aptamers have been selected for chlorpyrifis, acetamiprid, and several organophosphorus pesticides for example. ${ }^{241-243}$ These aptamers could be used to sequester pesticides inside a smart material, limiting their exposure to the surrounding environment.

Additional selections of aptamers binding important exudate targets would accelerate their application to agricultural problems. Organic acids have been shown to improve phosphorus availability, and many crops show an increase in organic acid exudation in response to low phosphorus. ${ }^{132}$ These organic acids could be useful targets for aptamer-based phosphate smart fertilizers. Allelochemical exudates could be used as aptamer targets for specific pesticide release. Allelochemical 6-methoxy-benzoxazolin-2-one (MBOA) has been shown to be released from wheat in response to encroaching weeds. ${ }^{244}$ Detecting MBOA in soil using an aptamer could allow for pesticide release on-demand. As more exudate compounds are identified and their specific functions elucidated, there is increased opportunity for exudate-binding aptamer smart materials. Creating controlled-release fertilizers and pesticides using exudate-binding 
aptamers could reduce their environmental impact, and could allow for more efficient, cropspecific fertilizer and pesticide products.

\subsection{Creating a Smart Fertilizer}

\subsubsection{Design of a smart fertilizer system}

As discussed in section 1.3.1, a large portion of the fertilizer nitrogen applied to crops is not taken up by the crop, but lost to the surrounding environment. The rate of nitrogen uptake by crops is variable over the course of the growing season, from year to year, and depending on

location. ${ }^{245}$ Nitrogen present in times of lower nitrogen uptake is at greater risk of leaching into the environment. The ability to synchronize the release of nitrogen with its uptake by crops would eliminate the need to apply a large excess, and thus decrease its environmental impact. It has been shown that the composition of root exudates varies under different nutrient availability. ${ }^{138}$ The identification of a root exudate that is released by crops in periods of increased nitrogen uptake would provide a target for aptamer selection. This exudate-binding aptamer could then be incorporated into a smart fertilizer system. A proposed aptamer smart fertilizer system is shown in Figure 1.6. 
A)

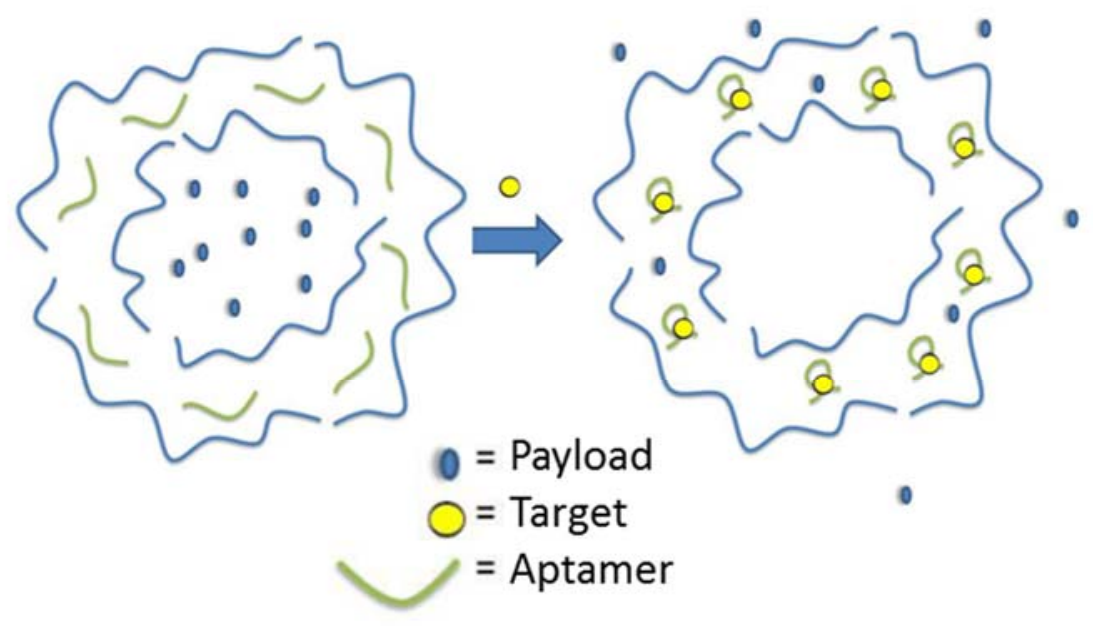

B)
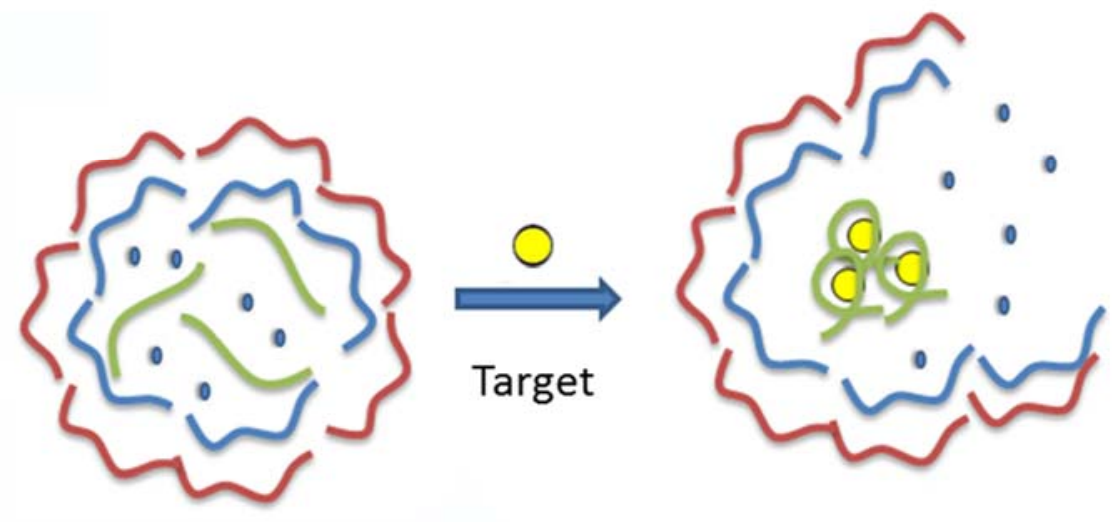

Figure 1.6: Proposed designs for an aptamer smart fertilizer. A) An aptamer (green) is embedded in the walls of a polyelectrolyte (blue lines) capsule, containing a nutrient payload (blue oval). Upon recognition of an exudate (yellow), the aptamer binds the exudate, causing the permeability of the capsule to increase, causing a slow release of the nutrient payload. B) An aptamer (green) is embedded inside a polyelectrolyte capsule (blue and red lines), acting as a scaffold. Upon recognition of the exudate (yellow), the aptamer binds, causing the capsule to burst/collapse, and the release of the nutrient payload. Figure reproduced from Mastronardi et al. 2014 in accordance with the Sensors Creative Commons Attribution License (CC BY 3.0). ${ }^{46}$

Before the exudate-binding aptamer could be selected and incorporated into an aptamer smart fertilizer system, several factors had to be confirmed. An exudate had to be identified which coincided with periods of increased nitrogen uptake by crops. The aptamer's ability to recognize and bind its target had to be maintained when the aptamer was embedded in a polymer film or capsule. Finally, the aptamer-target binding event had to lead to a physical change in the 
films and capsules allowing for the delivery of a payload. The progress towards these goals which led to the selection of an exudate-binding aptamer is described in section 1.5.2.

\subsubsection{Progress towards smart fertilizer development}

\subsubsection{Identifying root exudates synchronized with nitrogen uptake}

Dr. Carlos Monreal's group at Agriculture and Agri-Food Canada worked to identify root exudates that had an increased presence in soil when wheat and canola crops were taking up nitrogen. The Monreal group looked at three experimental groups: 1) soil alone, 2) soil with crop, without nitrogen, and 3) soil with crop, with nitrogen. The soil composition as well as nitrogen uptake by the crops was characterized weekly, and compared to the soil control in order to identify exudates which had a higher soil concentration while crops were taking up nitrogen. Dr. Monreal used mass spectrometry to examine soil components, and was able to identify which soil components were exuded from the crops by using isotopically labeled carbon dioxide and nitrogen gases in the greenhouse. These experiments allowed the Monreal group to identify root exudates from wheat and canola which had an increased presence in soil during periods of nitrogen uptake, shown in Figure 1.7. 

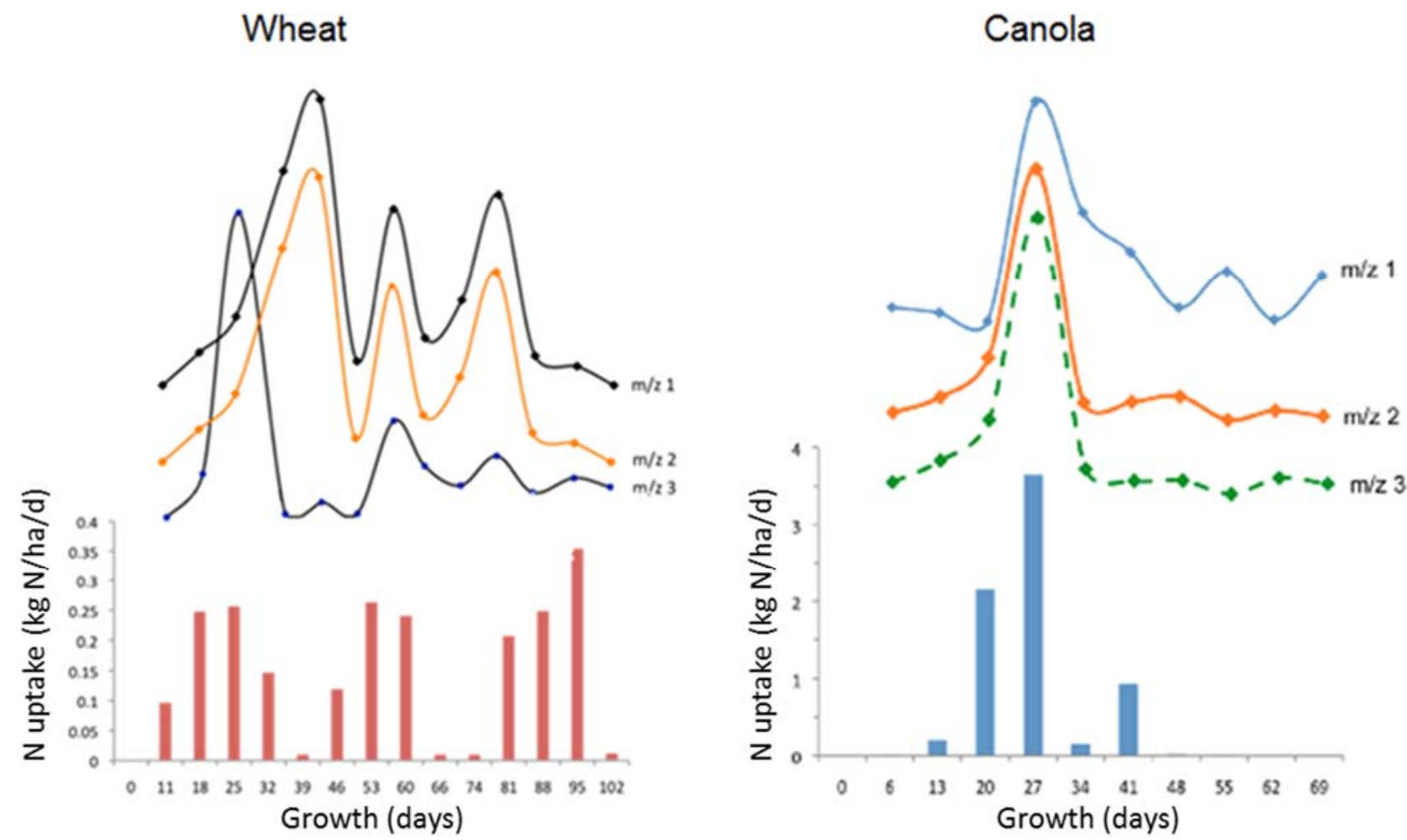

Figure 1.7: Soil components $(\mathrm{m} / \mathrm{z} 1-3)$ with increased presence in the soil during periods of increased nitrogen uptake by wheat and canola. Soil components were identified using mass spectrometry, and were determined to have been exuded from the crops by using isotopically labeled $\mathrm{CO}_{2}$, and $\mathrm{N}_{2}$ in the greenhouse. Experiments were performed by the Monreal group at Agriculture and Agri-Food Canada, and the figure was provided by Dr. Carlos Monreal.

Figure 1.7 shows that wheat had three periods of increased nitrogen uptake, while canola had one. The Monreal group examined the exudates present in the soil over several weeks of growth, and found that the presence of the amino acid L-serine in the soil was synchronized with these periods of nitrogen uptake. L-serine was the exudate suggested by Dr. Carlos Monreal to be used for aptamer development.

\subsubsection{Confirming aptamer-target binding in thin films}

With the design shown in Figure 1.6 in mind, it was important to confirm that an aptamer would still be able to bind its target when the aptamer was embedded in a thin film. Polyelectrolyte multilayer (PEM) films can be constructed using the Layer-by-Layer (LbL) 
technique, which involves the deposition of oppositely charged polyelectrolytes onto a charged surface. ${ }^{246}$ The adsorption of alternating positively and negatively charged polymers allows the multilayer film to be held together through electrostatic interactions, as well as allowing the thickness of the films to be controlled. As aptamers contain a negatively charged backbone, they are well-suited for incorporation into PEM films. A DNA aptamer was first incorporated into a PEM film by Sultan et.al. in 2009. ${ }^{247}$ An aptamer binding the dye sulforhodamine B (SB) was still able to recognize its target when embedded in a PEM film, with no loss of specificity and only a slight reduction in binding affinity. ${ }^{247}$ The sulforhodamine B aptamer was known to form a G-quadruplex structure, requiring $\mathrm{K}^{+}$for target-binding. ${ }^{248}$ To examine the aptamer's ability to bind the target SB dye, the dye's absorbance was examined after incubation with aptamer-PEM films as well as control PEM films. The sulforhodamine B aptamer films (SA) were examined with and without $\mathrm{K}^{+}$, and were compared to control films, which used calf thymus DNA (CT) or a random oligonucleotide $(\mathrm{RO})$ in place of the aptamer, and the results are shown in Figure 1.8. 


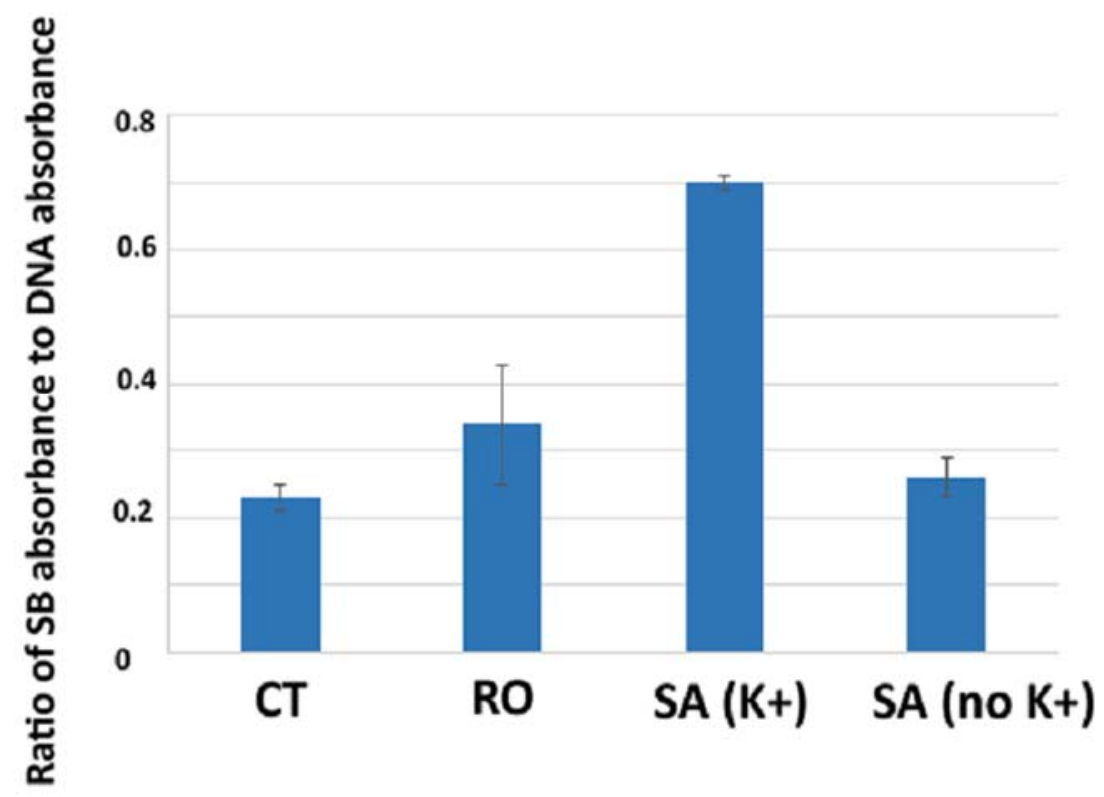

Figure 1.8: Target-binding of the sulforhodamine B aptamer embedded in PEM films. The ratio of the $U V$-vis absorbance of $S B$ dye $(570 \mathrm{~nm})$ to the absorbance of DNA $(260 \mathrm{~nm})$ is shown for aptamer (SA), random oligonucleotide (RO), and calf thymus (CT) embedded PDDA/PSS PEM films. The films were exposed to $2 \mathrm{mM}$ SB dye in either the absence or presence of $\mathrm{K}^{+}$, which is required for target binding. In the absence of $\mathrm{K}^{+}$, the $\mathrm{SA}$ embedded film shows no increase in target binding over the controls. In the presence of $\mathrm{K}^{+}, 2 \mathrm{x}$ better target binding is observed in the $\mathrm{SA}$-embedded films. Each ratio represents the average of at least 3 measurements, and the standard deviation is shown as error bars. Figure produced with data from Sultan et al. 2009. ${ }^{247}$ Reprinted from Mastronardi et al. 2016 with permission from Elsevier. ${ }^{249}$

In the absence of the $\mathrm{K}^{+}$needed for target-binding, the SA films showed no increase in target-binding compared to the control films. In the presence of $\mathrm{K}^{+}$, the SA films showed a $2 \mathrm{x}$ increase in target-binding, which indicated that the aptamer was able to form a G-quadruplex and bind its target while embedded in a PEM film. Aptamers for a variety of targets, such as quinine, D-arginine vasopressin, and bisphenol A, have since been incorporated into PEM films, described in Table 1.4. 
Table 1.4: Aptamers that have been incorporated into PEM films and microcapsules to generate smart material systems. The application and responsiveness of each system is described. Reprinted from Mastronardi et al. 2016 with permission from Elsevier. ${ }^{249}$

\begin{tabular}{|c|c|c|c|}
\hline Molecule Detected & Application & Responsiveness & Reference \\
\hline \multirow{5}{*}{$\begin{array}{l}\text { Sulforhodamine B } \\
\text { (SB) }\end{array}$} & Films & $\begin{array}{l}2 \mathrm{x} \text { increase in target binding over } \\
\text { control films }\end{array}$ & $\begin{array}{l}\text { Sultan et al., } \\
2009^{247}\end{array}$ \\
\hline & Films & $\begin{array}{l}2 \mathrm{x} \text { increase in diffusion of a redox } \\
\text { active probe over control films }\end{array}$ & $\begin{array}{l}\text { Sultan and } \\
\text { DeRosa, } 2011^{250}\end{array}$ \\
\hline & Microcapsules & $\begin{array}{l}\sim 8 \mathrm{x} \text { increase in diffusion of target } \\
\text { over control microcapsules }\end{array}$ & $\begin{array}{l}\text { Sultan and } \\
\text { DeRosa, } 2011^{250}\end{array}$ \\
\hline & LSPR sensor & $\begin{array}{l}\text { Est. } 2 \mathrm{x} \text { increase in diffusion of } \\
\text { etchant over control films }\end{array}$ & $\begin{array}{l}\text { Malile et al., } \\
2013^{251}\end{array}$ \\
\hline & $\begin{array}{l}\text { Biodegradable } \\
\text { films }\end{array}$ & $\begin{array}{l}2 \mathrm{x} \text { increase in target binding over } \\
\text { control films }\end{array}$ & $\begin{array}{l}\text { Foster and } \\
\text { DeRosa, } 2014^{82}\end{array}$ \\
\hline Quinine & LSPR sensor & $\begin{array}{l}\text { Est. } 4 \mathrm{x} \text { increase in diffusion of } \\
\text { etchant over control films }\end{array}$ & $\begin{array}{l}\text { Malile et al., } \\
2013^{251}\end{array}$ \\
\hline Lysine & Microcapsules & $\begin{array}{l}\sim 2 \mathrm{x} \text { increase in diffusion of a } \\
\text { fluorescent dye over control } \\
\text { microcapsules }\end{array}$ & $\begin{array}{l}\text { Sultan and } \\
\text { DeRosa, 2011 }\end{array}$ \\
\hline $\begin{array}{l}\text { D-Arginine } \\
\text { Vasopressin (D-VP) }\end{array}$ & $\begin{array}{l}\text { Electrochemical } \\
\text { sensor }\end{array}$ & LOD $1 \mathrm{ng} / \mathrm{mL}$ & Qin et al., $2012^{252}$ \\
\hline Bisphenol A (BPA) & ECL sensor & $\begin{array}{l}\text { Increased diffusion of a coreactant } \\
\mathrm{S}_{2} \mathrm{O}_{8}{ }^{2-} \text { required for ECL, leading to a } \\
64 \mathrm{x} \text { increase in ECL intensity over } \\
\text { control film; LOD } 0.05 \mathrm{ng} / \mathrm{mL}\end{array}$ & $\begin{array}{l}\text { Chen et al., } \\
2015^{253}\end{array}$ \\
\hline
\end{tabular}

1 Estimated from Figure $2 \mathrm{~b}$ from Malile et al. $2013^{251}$

2 Estimated from Figure $4 \mathrm{~b}$ from Malile et al. $2013^{251}$

When the aptamers were embedded into PEM films, not only could they recognize and bind their target, but they could make the films responsive, by causing a change in film permeability upon target-binding. Using the sulforhodamine B aptamer, aptamer-PEM films were deposited onto an electrode, and the diffusion of a redox-active probe through the films after target-binding was compared to control films. ${ }^{247}$ Figure 1.9 shows the diffusion of this redox active probe through SA-films, compared to films containing no DNA, and a film containing a random oligonucleotide (RO). 


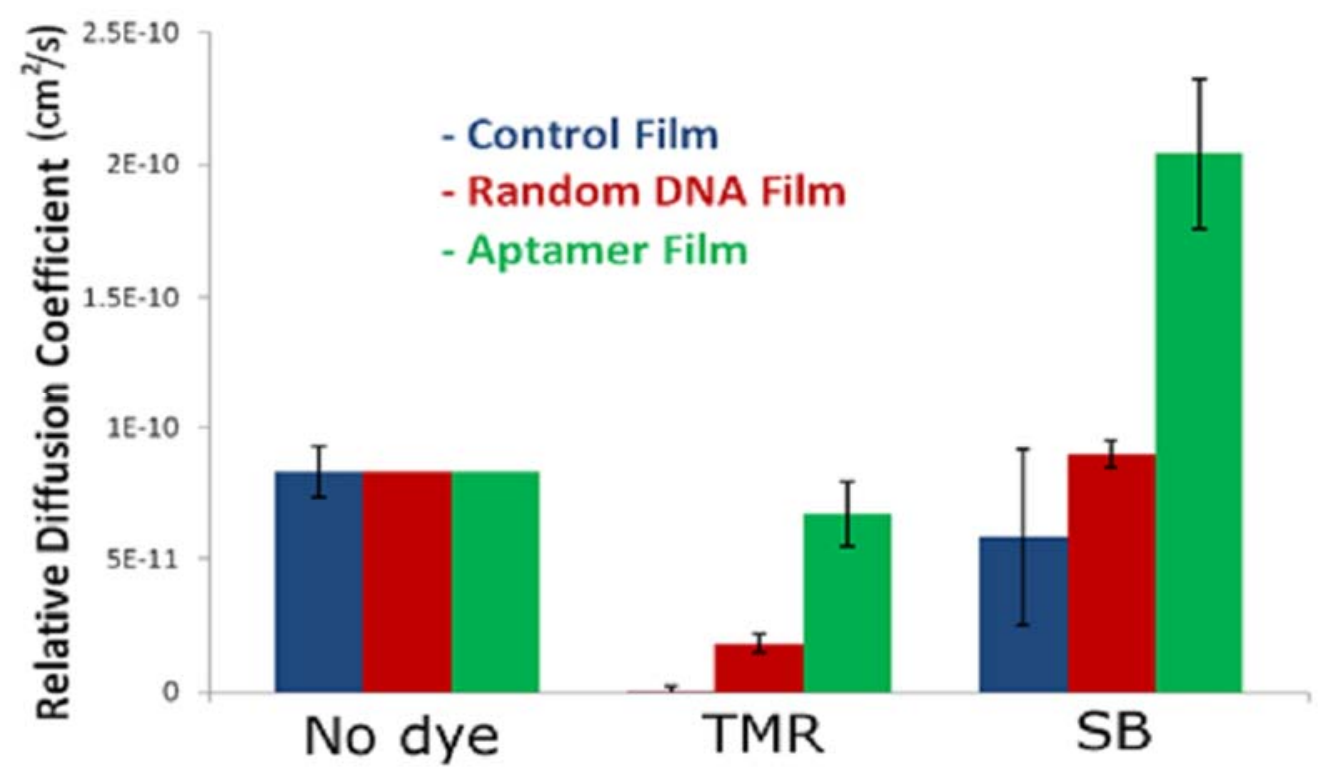

Figure 1.9: Examination of PEM film permeability to a redox active probe. Three types of PEM films were examined in triplicate: a film containing only PAH/PSS (blue), ROembedded PAH/PSS (red), and SA-embedded PAH/PSS (green). The diffusion coefficient for the redox active probe $\left[\mathrm{Ru}\left(\mathrm{NH}_{3}\right)_{6}\right]^{3+/ 2+}$ was measured under three conditions for each film: no target present (no dye), in the presence of a structural analog of the target (TMR), and in the presence of the aptamer's target (SB). All diffusion coefficients were normalized to the values measured under "no dye" conditions. A $2 x$ increase in diffusion coefficient was only observed when the aptamer film was exposed to its target, SB. Figure produced with data from Sultan et al. 2009. ${ }^{247}$ Reprinted from Mastronardi et al. 2016 with permission from Elsevier. ${ }^{249}$

The permeability was examined in the presence of the target sulforhodamine B (SB), a structural analog tetramethylrosamine (TMR), and in the absence of any target molecule. A significant increase in diffusion was observed in the aptamer-PEM after incubation with the target SB, compared to the control films. The structural analog TMR did not cause a change in film permeability, demonstrating the specificity of the aptamer-PEM response. The ability of the PEM films to undergo a physical change in response to aptamer-target binding could allow them to move beyond sensing, into controlled delivery. 


\subsubsection{Creating aptamer-responsive capsules}

Section 1.5.2.2 described the success of incorporating aptamers into PEM-films. The proposed aptamer smart fertilizer design in Figure 1.6 was envisioned as a capsule, so the LbL method was employed using a sacrificial calcium carbonate core $\left(\mathrm{CaCO}_{3}\right)$ as a template, to generate PEM microcapsules with aptamers either embedded in the walls, or as a scaffold at their core, as described in Figure 1.10. These strategies were both successfully employed using the sulforhodamine B aptamer, generating responsive microcapsules. ${ }^{250,254}$ 


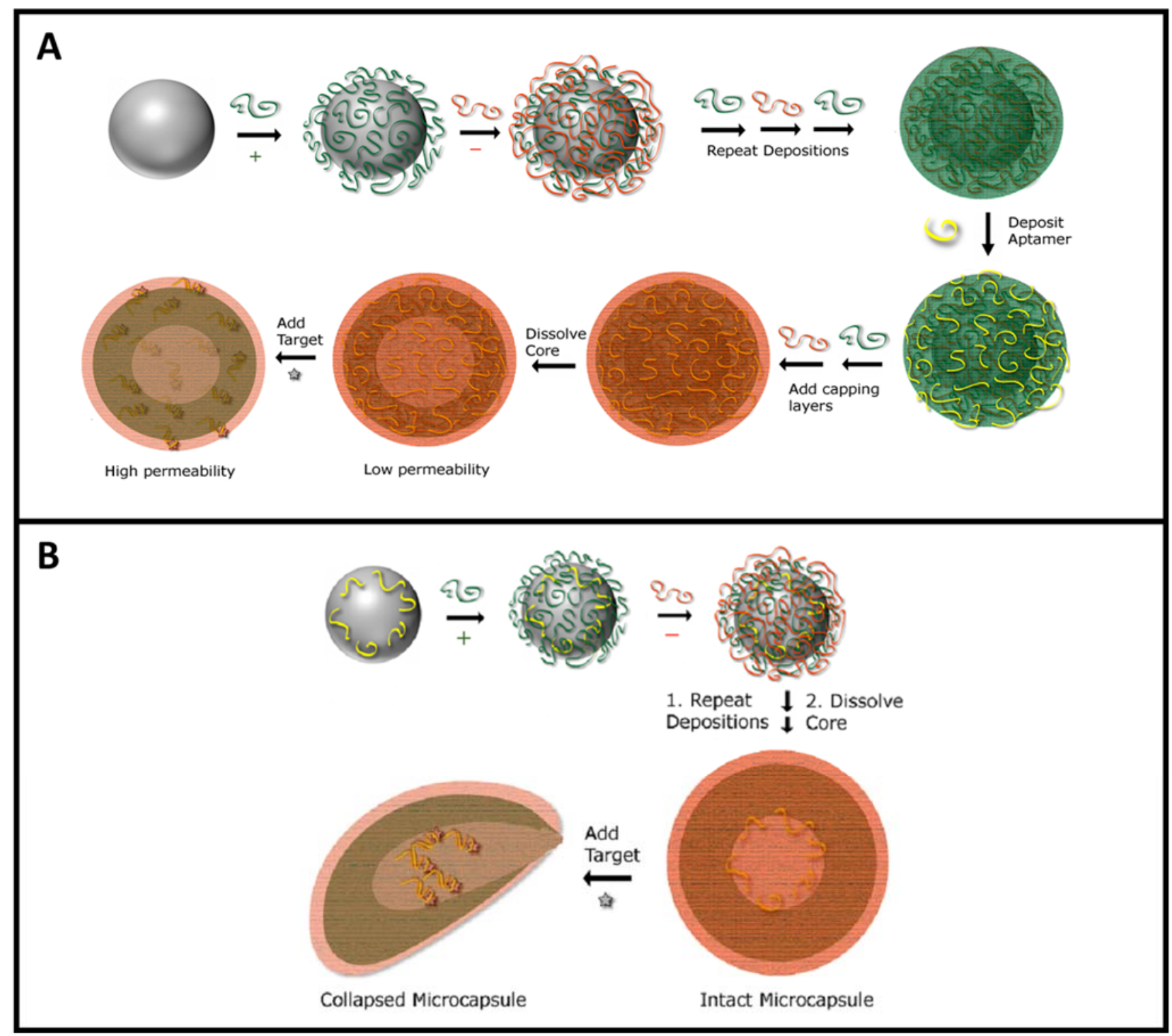

Figure 1.10: Incorporation of aptamers into PEM microcapsules. Charged polyelectrolytes (green and orange ribbons) are alternatively deposited onto a sacrificial spherical template $\left(\mathrm{CaCO}_{3}\right)$ that gets dissolved, leaving hollow microcapsules. Aptamers can be incorporated in two ways. A) After a desired number of base layers, an aptamer (yellow ribbons) can be substituted for the negatively charged polyelectrolyte for a desired number of aptamercontaining bilayers. An additional polyelectrolyte bilayer can then be added as a capping layer. In the absence of the aptamer's target, the microcapsules have limited permeability. Aptamer-target binding leads to increased permeability. B) The sacrificial spherical template $\left(\mathrm{CaCO}_{3}\right)$ has been doped with aptamer (yellow ribbon). After dissolution of the core, the aptamers act as a scaffold within the microcapsule. In the absence of the target, the microcapsule is stable. Aptamer-target binding causes the collapse of the microcapsule. Reprinted from Mastronardi et al. 2016 with permission from Elsevier. ${ }^{249}$ 
Using the strategy shown in Figure 1.10 A, Sultan and DeRosa incorporated the sulforhodamine B aptamer into the walls of polyelectrolyte microcapsules. ${ }^{250} \mathrm{By}$ using a fluorescein-tagged aptamer, confocal laser scanning microscopy (CLSM) could be used to examine the fluorescence co-localization of the aptamer with its target dye. The co-localization of the tagged aptamer with sulforhodamine B could only be observed in the presence of $\mathrm{K}^{+}$, which is required for its G-quadruplex formation and target-binding. As was seen with the aptamer-PEM films, the aptamer was able to form a G-quadruplex and bind its target when embedded in the walls of PEM-microcapsules.

For these microcapsules to be used for controlled delivery, there would need to be a change in permeability upon target-binding that would allow the release of a cargo. To investigate this, Sultan and DeRosa used fluorescence recovery after photobleaching (FRAP) to measure the diffusion constant for the movement of SB through multiple PEM-microcapsules. ${ }^{250}$ The diffusion of SB through aptamer-PEM-microcapsules was compared to its diffusion through PEM-microcapsules made with a random oligonucleotide, and with polyelectrolytes alone. The diffusion of the SB target molecule increased $8 \mathrm{x}$ in the aptamer-PEM-microcapsule compared to the control microcapsules, indicating that aptamer-target binding increased the permeability of the PEM microcapsule. Sultan and DeRosa used the L-lysine aptamer in place of the sulforhodamine B aptamer in the PEM-microcapsules, to examine the diffusion of SB as a nontarget molecule in the presence and absence of the aptamer's target, L-lysine. The L-lysine aptamer-PEM microcapsules became 2x more permeable to SB in the presence of the aptamer's target, L-lysine, compared to L-histidine. This finding showed that aptamer-target binding created an increase in permeability of the microcapsule wall that could be exploited to release a cargo on demand. 
Zhang et al. created sulforhodamine B aptamer-PEM microcapsules using the strategy

outlined in Figure 1.10 B. ${ }^{254}$ Instead of using the aptamers as a negatively charged

polyelectrolyte layer, the sulforhodamine $\mathrm{B}$ aptamers were doped into the $\mathrm{CaCO}_{3}$ core, and upon dissolution of this template, the aptamers were left in the interior of the microcapsules, acting as a scaffold. When these microcapsules were incubated with the SB target over several days, the microcapsules were found to rupture and burst, shown in Figure 1.11. An incubation of the capsules in buffer alone or a similar dye molecule, TMR, did not lead to structural changes of the microcapsules. This finding suggested that aptamer-target binding caused the collapse and rupture of these microcapsules. These aptamer-loaded PEM microcapsules could be useful for one-time burst release of a desired payload.
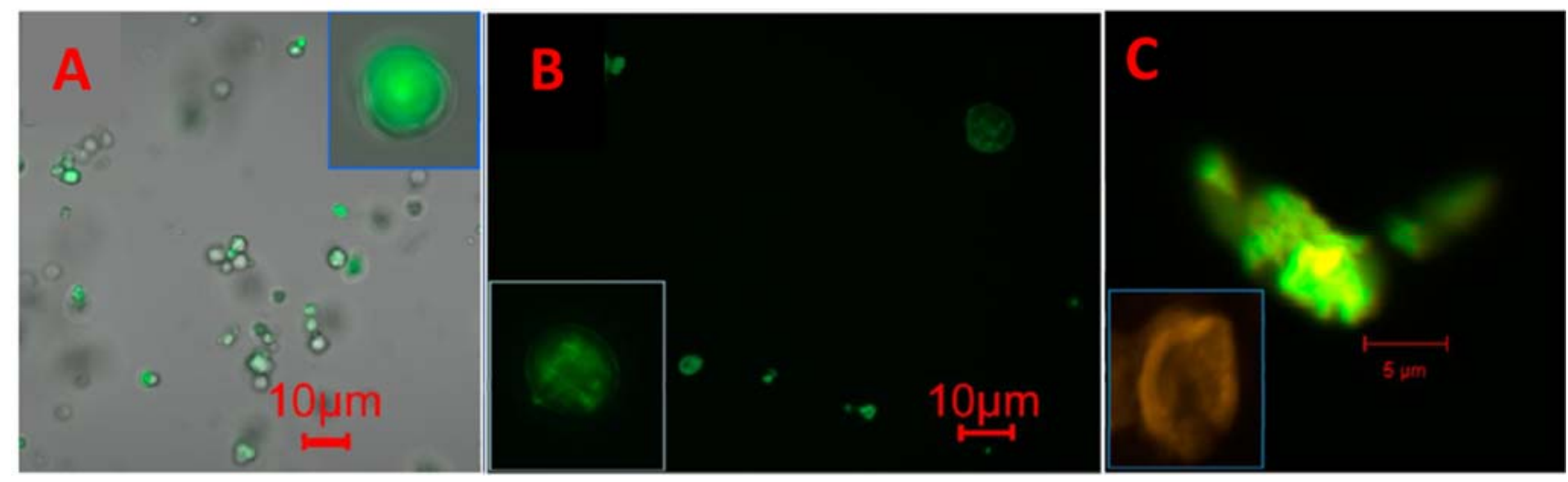

Figure 1.11: Aptamer-responsive polyelectrolyte microcapsules. A) Confocal laser scanning microscopy (CLSM) image of sulforhodamine B aptamer (SA): polystyrene sulfonate (PSS)- $\mathrm{CaCO}_{3}$ microcapsules prepared with fluorescein-labeled aptamers. B) CLSM image of SA:PSS-(Polydiallyldimethylammonium chloride (PDDA)/PSS)5 microcapsules prepared from fluorescein-labeled aptamers. C) Colocalization experiment showing aptamer-target binding caused microcapsule rupture after 6 days. Sulforhodamine B target at $1 \mathrm{mM}$ and $0.1 \mathrm{M} \mathrm{KCl}$ was used. Inset image shows ruptured non-fluorescent aptamer microcapsule. Modified with permission from ACS Publications. ${ }^{254}$ 


\subsection{Thesis objectives}

The main objective of this work was to investigate improvements for small molecule aptamer selections while selecting and characterizing novel L-serine-binding DNA aptamers for use in a smart fertilizer system. A simple DNA-lipid conjugate was also investigated for potential use in targeted delivery applications.

In chapter 2, "Selection of L-serine-binding aptamers using multiple selection strategies", SELEX experiments generating L-serine-binding DNA aptamers were described. Three separate starting libraries, including a novel library, a library derived from a randomized L-homocysteine aptamer, and a library redirected from an L-homocysteine SELEX experiment, were examined for their effect on producing L-serine-binding aptamer candidates. High-throughput sequencing was used to identify aptamer candidates, and sequence homology was examined for possible Lserine-binding sites.

In chapter 3, "Characterization of L-serine-binding aptamers", aptamer candidates identified in Chapter 2 were examined for their ability to bind immobilized and free L-serine. Minimer sequences were designed from the most promising candidate, and their binding affinities were examined. A Spiegelmer was synthesized to investigate the aptamer-target binding interactions, as well as to increase the aptamer's resistance to nuclease degradation, allowing it to be more easily bridged into fertilizer applications.

In Chapter 4, "Synthesis and characterization of aptamer-polymersomes", an aptamerlipid conjugate system was synthesized for potential targeted delivery applications. Lipids of varying lengths were investigated for their effect on polymersome size and stability. Target- 
binding of the aptamer when incorporated into aptamer-lipid conjugates and aptamerpolymersomes, as well as its effect on polymersome structure were also studied. 
2 Selection of L-serine-binding aptamers using multiple selection strategies 


\subsection{Chapter Objectives}

In this chapter, DNA aptamers for the small molecule crop exudate, L-serine, were developed for use in a smart fertilizer system. The evaluation of aptamer Hcy10 for L-serine binding, and the selection of novel aptamers for L-serine were described. The objectives were twofold: to generate promising L-serine-binding aptamer candidates, and to use three different starting libraries to assess whether small molecule selections could be expedited using pools enriched for a similar small molecule, L-homocysteine.

\subsection{Statement of contributions}

The three SELEX starting libraries were conceptualized by E. Mastronardi and M.C. DeRosa, based on the L-homocysteine selection performed by M. McKeague. DNA synthesis was performed by E. Mastronardi, as well as all SELEX experiments for L-serine. Soil solution was obtained from C. Monreal at Agriculture and Agri-Food Canada. High-throughput sequencing samples were prepared by E. Mastronardi and run by Genome Quebec. Analysis of high-throughput sequencing data was done by E. Mastronardi with assistance from E. Hassan and J. Hoinka. 


\subsection{Introduction}

\subsubsection{SELEX for small molecules}

Small molecule targets were the initial focus of the SELEX process, with the selection of RNA aptamers for small molecule dyes. ${ }^{14}$ Selections progressed using a variety of more complex targets, and aptamers were successfully generated against cell membranes in 1998 using red blood cells. ${ }^{255}$ Tan's group demonstrated that SELEX could be used to identify disease markers on whole cells, generating aptamers specific for leukemia cells, and interest in this area for cancer research grew steadily, with less focus on small molecule targets. ${ }^{19}$

Many biologically and environmentally relevant targets are small molecules including disease markers, toxins, heavy metals, and plant exudates. Rapid identification of these small molecule targets could aid the medical community to more quickly make diagnoses, and help food safety workers quickly identify toxins and food contaminants, for example. Though the majority of aptamers are selected against protein and whole cell targets, interest in small molecule selections is starting to increase, with aptamers selected against antibiotics, mycotoxins, and drugs for example. ${ }^{256}$ Figure 2.1 shows that while most aptamers are selected against protein targets, the amount of selections against small molecule targets has increased from $19 \%$ to $25 \%$ from 2011 to $2013 .^{18,24}$ 

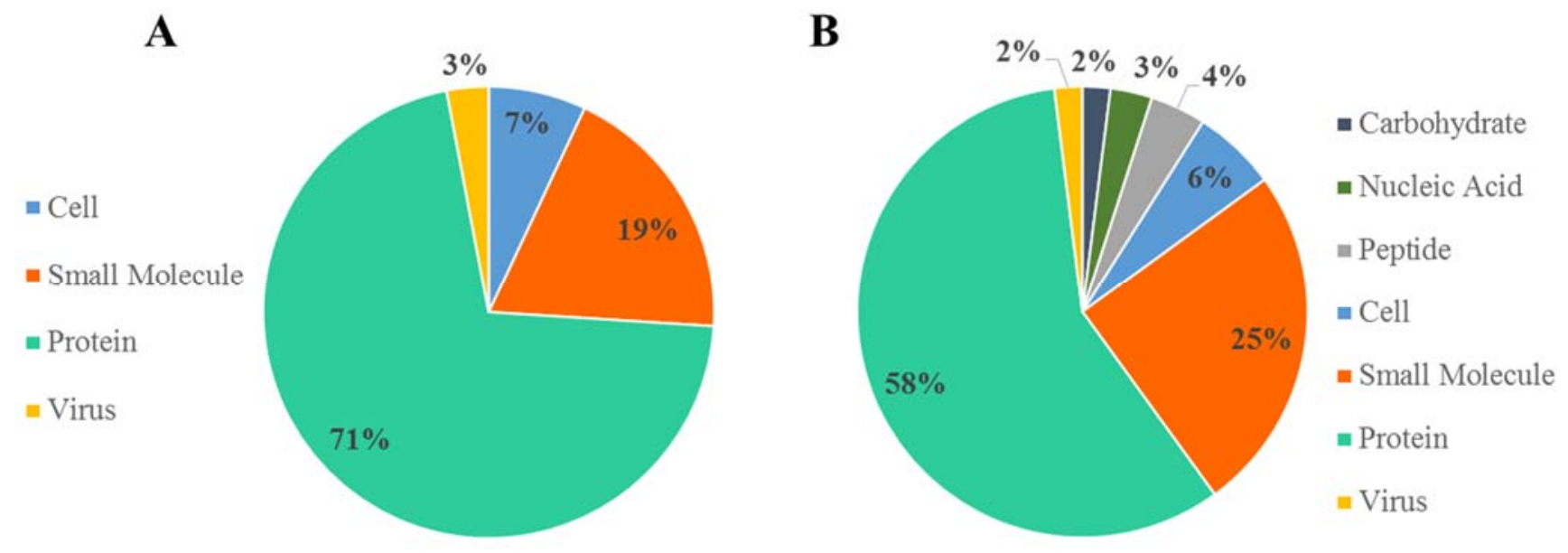

Figure 2.1: Target types of aptamers selected between (A) 1990 and 2011, and (B) 1990 and 2013. Target-types were refined to include additional categories in (B) Figure created using data from McKeague et al. 2012, and McKeague et al. 2015. 18,24 $^{2}$

Aptamers are promising candidates for molecular recognition elements for small

molecules, as there are fewer restraints on their selection conditions than antibodies. Unlike antibodies, aptamers can be developed against toxins as their in vitro production does not require animals. ${ }^{256,257}$ Aptamers can also be generated against small molecules that do not elicit the immune response needed for antibody production. Their chemical synthesis allows aptamers to be easily modified for incorporation into sensing platforms.

\subsubsection{Challenges in aptamer selection for small molecules}

Due to their size, small molecules provide fewer functional groups for aptamer binding compared to proteins and whole cells, making them a challenging target for aptamer selection. From an analysis of all selections completed between 1990 and 2013, aptamers for small molecules showed a lower affinity to their target on average than aptamers for larger targets. ${ }^{18}$ Though this may make small molecules a less desirable target, the reasons for this lower affinity are not understood, and could stem from the difficulty in accurately measuring the affinity of 
aptamers for small molecules. Challenges in measuring this affinity are discussed in greater detail in section 3.3.2.

The size of small molecules poses some challenges for aptamer selection, particularly in the partitioning step of the SELEX process. Partitioning unbound target from the oligonucleotide-target complexes is a crucial step in the selection process in order to isolate target-binding sequences. Protein targets can often be effectively separated from the oligonucleotide pool using nitrocellulose filters. ${ }^{24}$ Cell targets can be separated from oligonucleotides using fluorescence activated cell-sorting (FACS), or through washing. ${ }^{258}$ When oligonucleotides bind a small molecule target, the mass of the complex is not much larger than the oligonucleotide alone, often making filtration ineffective.

To ensure efficient partitioning, the target is often immobilized during the selection steps, allowing oligonucleotides with no affinity to be washed away. This immobilization step introduces some difficulty, as specific functional groups are required for coupling to a solid support, limiting the target and support options. Coupling the target to a solid support makes at least one functional group on the target molecule unavailable for interaction with the oligonucleotide library, which could be significant on a small molecule with few functional groups. Many solid support options such as sepharose, agarose, or magnetic beads can add very bulky or charged groups to the target, interfering in the selection as these groups can compete with the small molecule for aptamer binding. ${ }^{24}$ Oligonucleotides with affinity for the solid support will be difficult to eliminate throughout the selection, making it less clear which sequences have affinity for the target. At the end of the selection, some aptamer sequences may show affinity for the target only in its immobilized form, as aptamers could be binding to both the target and functional groups provided by the solid support. This partial binding to the solid 
support could prove difficult for downstream aptamer applications if a target was to be analyzed in solution. For example, an aptamer selected for the dye sulforhodamine B showed a higher binding affinity with the dye immobilized to agarose than for the dye free in solution, suggesting the aptamer had some affinity for the agarose. ${ }^{248}$ Though immobilizing the target has many drawbacks, a careful application of control rounds can help minimize the interference of solid supports.

Rather than immobilizing the target, SELEX using an immobilized oligonucleotide library, called Capture-SELEX, has also been described. ${ }^{259,260}$ In this process, a docking sequence is inserted into the random region of the oligonucleotide library. A short oligonucleotide probe, complementary to the docking sequence, is immobilized on magnetic beads, allowing for hybridization to the library sequences. Upon incubation with the target, oligonucleotides with high affinity for the target molecule will be released from the beads, while non-binding sequences remain hybridized on the magnetic beads. ${ }^{260}$ This Capture-SELEX process has been used successfully for small molecule antibiotics and drugs. ${ }^{260-262}$

\subsubsection{High-throughput Sequencing (HTS)}

Until recently, aptamer candidate sequences were identified by cloning and sequencing the final selection round, using traditional cloning methods and Sanger sequencing. ${ }^{29}$ After many selection rounds, around a hundred clones were typically sequenced, generating a few sequences which were most abundant in the final selection round. ${ }^{29}$ Since few sequences could be obtained, the enrichment of each selection round was monitored, and SELEX was often continued until a plateau in target-binding was reached, or the majority of the pool bound the target. This method

yielded few sequences, relying largely on chance that the best target-binding sequence would be found. The low sequence number obtained after cloning ran the risk of poorly representing the 
pool diversity, with high copy number sequences, and sequences with high cloning efficiency being favoured. Using traditional cloning and sequencing methods, there was no way to determine if abundant sequences were a result of their enrichment or PCR amplification bias. With the many challenges of aptamer development for small molecules, this low-throughput method of obtaining aptamer candidates could be a contributing factor to the low success rate of small molecule aptamer selections..$^{24,263}$

High-throughput sequencing has been a staple in genomic studies since it was introduced in 2005, and has more recently been applied to aptamer development. ${ }^{264,265}$ In high-throughput SELEX (HT-SELEX), part of the selection pool is used for sequencing while the rest is used as the starting pool for the next SELEX round. Sequencing every round or every few rounds through the selection process allows the selection to be computationally monitored, and lowers the number of selection rounds needed to find aptamer candidates. ${ }^{266}$ Kupakuwana et al. proposed that aptamers could be identified after a single SELEX round using high-throughput sequencing. ${ }^{265}$ Importantly, high-throughput sequencing provides millions of sequences increasing the likelihood of identifying tight binders compared to traditional cloning. During SELEX experiments, high-binding sequences are enriched, while low or non-binding sequences are depleted. High-throughput sequencing allows us to track the progression of individual sequences as they are exposed to selective pressures. This facilitates the differentiation of sequences that have been enriched due to target-binding from sequences which may be overly represented from PCR bias. With HTS, sequences binding non-specifically to the solid-support that persist through the selection can also be eliminated, which is particularly helpful in small molecule selections. HTS was used to successfully identify aptamers for the amino-acid derivative tyramine, for example. ${ }^{267} \mathrm{HTS}$ can provide insight into which sequences are strong 
aptamer candidates and should be selected for further characterization, overcoming the reliance on chance provided by traditional cloning methods.

\subsubsection{Illumina sequencing}

The Illumina system for high-throughput sequencing is widely used, and allows for the detection of each nucleotide as it is added, through a process called Sequencing by Synthesis (SBS) ${ }^{268}$ The DNA to be sequenced first gets modified with Illumina adaptors, through PCR. These adaptors contain three parts: (1) nucleotides complementary to sequences attached to the flow cell; (2) a DNA "barcode" allowing each sequence to be traced back to the SELEX pool from which it came; and (3) a primer binding region allowing the DNA to be amplified. With the Illumina adaptors attached, the DNA libraries are sent through a flow cell and can bind the complementary sequences on the flow cell surface. Bridge amplification allows each sequence to be replicated numerous times, leading to the formation of millions of clusters containing individual sequences. A denaturation step leaves only the desired sequences bound to the flow cell, and sequencing by synthesis can begin. The addition of four fluorescently-labeled nucleotides with reversible terminators allows the sequence of each cluster to be measured in parallel. During each cycle, a laser excites each fluorophore, and the emitted fluorescence allows the base to be identified in each cluster. The terminators are removed and this cycle is repeated to determine the entire sequence in each cluster. Millions of sequences are analyzed, and are read using sequence alignment software.

\subsubsection{AptaCluster}

As high-throughput sequencing generates millions of sequences, it is essential to have a computational system able to analyze such large amounts of data. Most computational processing tools for these libraries focus on identifying the sequences with the highest copy 
number in the final round of selection. ${ }^{269}$ As SELEX is an in vitro evolutionary process, highbinding sequences could be introduced through PCR mutations, and would be missed through this kind of analysis alone. Disproportionate counts could also result from highly structured sequences which amplified at a lower rate. New computational tools have been developed specifically for analysis of SELEX experiments, such as FASTAptamer, and AptaCluster. ${ }^{269,270}$ These tools not only monitor the total number of each sequence, but the enrichment of each sequence from round to round. AptaCluster is able to analyze whole SELEX pools (2-50 million sequences per cycle), giving a global overview of selection progression and enabling the identification of higher quality aptamer candidates. ${ }^{269}$

\subsubsection{Rationale for 3 SELEX pools}

SELEX can be a time consuming process, particularly for small molecule targets, and it has been estimated that less than $30 \%$ of SELEX experiments result in successful aptamers..$^{24,263}$ Additionally challenging for small molecule targets is the lower affinity generally observed for

these targets compared to aptamers for larger molecules. ${ }^{18}$ Many aptamers have been generated for amino acids, and are listed along with their binding affinities in Table 2.1. 
Table 2.1: Apparent dissociation constants for DNA and RNA aptamers selected for amino acid targets.

\begin{tabular}{|c|c|c|c|}
\hline $\begin{array}{l}\text { Amino Acid } \\
\text { Target }\end{array}$ & DNA/RNA & Apparent $K_{d}$ & Reference \\
\hline L-Arginine & $\begin{array}{l}\text { DNA } \\
\text { RNA }\end{array}$ & $\begin{array}{c}2.5 \mathrm{mM} \\
56-76 \mu \mathrm{M} \\
330 \mathrm{nM}\end{array}$ & $\begin{array}{l}\text { Harada et al. } 1995^{166} \\
\text { Famulok } 1994^{165} \\
\text { Geiger et al. } 1996^{167}\end{array}$ \\
\hline D-Arginine & RNA & $410 \mu \mathrm{M}$ & Famulok $1994^{165}$ \\
\hline $\begin{array}{c}\text { L- and D- } \\
\text { Glutamic Acid }\end{array}$ & DNA & $580-810 \mu \mathrm{M}$ & Ohsawa et al. $2008^{139}$ \\
\hline L-Histidine & RNA & $8-54 \mu \mathrm{M}$ & Majerfeld et al. $2005^{170}$ \\
\hline Isoleucine & RNA & $0.9 \mathrm{mM}$ & Legiewicz et al. $2005^{159}$ \\
\hline L-Isoleucine & $\begin{array}{l}\text { RNA } \\
\text { RNA }\end{array}$ & $\begin{array}{c}200-500 \mu \mathrm{M} \\
1-7 \mathrm{mM}\end{array}$ & $\begin{array}{l}\text { Majerfeld et al. } 1998^{160} \\
\text { Lozupone et al. } 2003^{161}\end{array}$ \\
\hline L-Phenylalanine & RNA & $34-55 \mu \mathrm{M}$ & Illangasekare et al. $2002^{173}$ \\
\hline L-Tryptophan & DNA & $1.757 \mu \mathrm{M}$ & Yang et al. $2011^{204}$ \\
\hline D-Tryptophan & RNA & $18 \mu \mathrm{M}$ & Famulok et al. $1992^{271}$ \\
\hline L-Tyrosine & RNA & $35 \mu \mathrm{M}$ & Mannironi et al. $2000^{158}$ \\
\hline L-Valine & RNA & $2.9 \mathrm{mM}$ & Majerfeld et al. $1994^{168}$ \\
\hline
\end{tabular}

The binding affinities observed in Table 2.1 for amino acid targets generally range from high micromolar to low millimolar which is consistent with the finding that aptamers have lower affinity for small molecule targets compared to other target types. ${ }^{18}$ To increase the chances of producing an L-serine-binding aptamer with good binding affinity, multiple SELEX experiments were conducted in parallel to utilize multiple selection strategies.

The goal of these experiments was not only to generate a successful L-serine-binding aptamer, but also to investigate methods to speed up the selection process. Other groups have successfully generated new aptamers from an existing aptamer sequence. For example, a citrulline-binding RNA aptamer was randomized $30 \%$ and used to generate an L-argininebinding aptamer. ${ }^{165}$ This same randomization strategy was used to generate an L-tyrosinebinding RNA aptamer from a dopamine-binding aptamer. ${ }^{158}$ In our lab, an aptamer had been 
generated for the small molecule amino acid L-homocysteine by a previous student. ${ }^{272}$ This Lhomocysteine selection underwent several negative selections, as well as counter selections against similar amino acids. These steps would also be beneficial in the selection of an L-serinebinding aptamer. With a goal of speeding up the selection process, the final rounds of the Lhomocysteine selection were used as one starting library for the L-serine selection (Redirected SELEX). An L-homocysteine aptamer generated from this previous selection also showed some affinity to L-serine, so a library was generated by randomizing this aptamer (Randomized SELEX). An original library was also used that had never been used in a selection (Original SELEX). The development of each starting library is described in the flow-chart in Figure 2.2. 


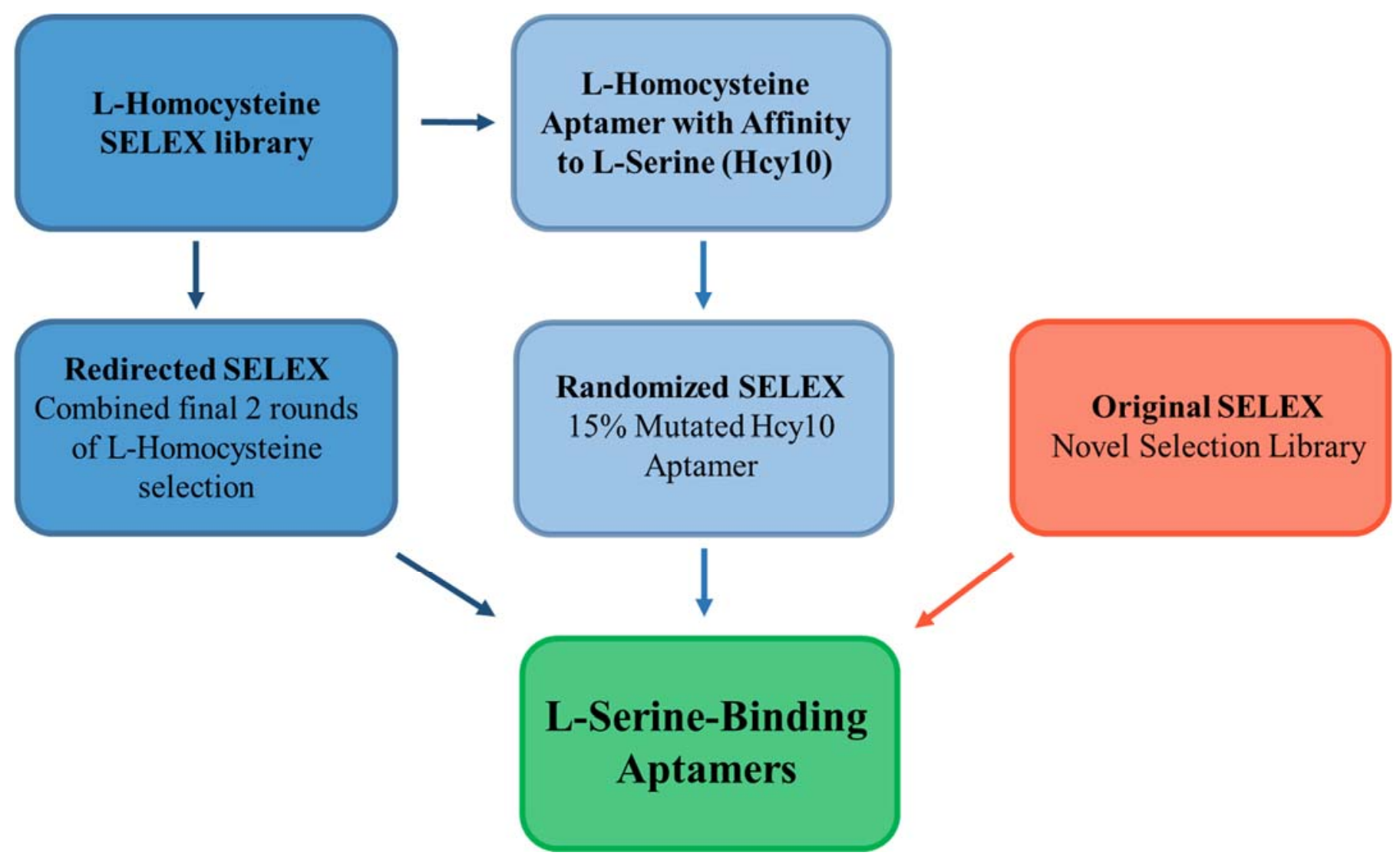

Figure 2.2: Three strategies used to generate L-serine-binding aptamers. From a previous SELEX for L-homocysteine, the Redirected SELEX, and Randomized SELEX starting libraries were generated. An Original SELEX library was also created.

\subsection{Materials and Methods}

\subsubsection{Chemicals and Reagents}

For SELEX experiments, NHS-activated Sepharose 4 Fast Flow, ninhydrin, and buffer components: sodium bicarbonate $\left(\mathrm{NaHCO}_{3}\right)$, sodium acetate $\left(\mathrm{CH}_{3} \mathrm{COONa}\right)$, sodium phosphate dibasic $\left(\mathrm{Na}_{2} \mathrm{HPO}_{4}\right)$, potassium phosphate monobasic $\left(\mathrm{KH}_{2} \mathrm{PO}_{4}\right)$, magnesium chloride $\left(\mathrm{MgCl}_{2}\right)$, and $\mathrm{HCl}$ were obtained from Sigma-Aldrich (Oakville, ON, Canada). Additional buffer components, sodium chloride $(\mathrm{NaCl})$, Tris, and urea, as well as target molecules, L-serine, Lglycine, and L-threonine were obtained from BioShop Canada (Burlington, ON, Canada). Costar 
Spin-X cellulose acetate centrifuge filters were obtained from Corning Incorporated (New York, United States).

DNA synthesis was carried out on a BioAutomation Corporation MerMade 6 oligonucleotide synthesizer using standard phosphoramidite chemistry. All amidites, modifiers, and reagents were obtained from Glen Research, $1000 \AA$ CPG $1 \mu \mathrm{mol}$ scale columns were obtained from BioAutomation, and synthesis grade acetonitrile was obtained from VWR. 28\% ammonium hydroxide solution was obtained from Sigma-Aldrich (Oakville, ON, Canada).

PAGE reagents: acrylamide/bis-acrylamide $40 \%$ solution, Tris, boric acid, EDTA and N,N,N',N'-tetramethylethylenediamine (TEMED), were obtained from BioShop Canada (Burlington, ON, Canada), while formamide and $3 \mathrm{kDa}$ cutoff Amicon Ultra centrifugal filter columns were obtained from Sigma-Aldrich (Oakville, ON, Canada). Reagents for PCR (25 mM $\mathrm{MgCl}_{2}, 10 \mathrm{mM}$ dNTP mix, and Taq DNA polymerase) were obtained from BioShop Canada (Burlington, ON, Canada)

\subsubsection{L-serine conjugation to sepharose beads}

NHS-activated Sepharose 4 Fast Flow resin was used to couple the target L-serine. All solutions used during coupling were cooled to $4^{\circ} \mathrm{C}$. The sepharose resin was washed with $10-15$ medium volumes of $1 \mathrm{mM} \mathrm{HCl} .115 \mathrm{mM}$ L-serine was dissolved in coupling buffer $(0.2 \mathrm{M}$ $\mathrm{NaHCO}_{3}, 0.5 \mathrm{M} \mathrm{NaCl}, \mathrm{pH} 8.3$ ) and mixed with the sepharose resin at a ratio of $0.5: 1$ coupling solution to resin. The coupling was allowed to take place for 4 hours on a shaker at room temperature. After coupling, the coupling solution was removed and set aside to verify coupling efficiency. Any unreacted groups were blocked using 0.1 M Tris- $\mathrm{HCl}$ on a shaker for 3 hours. After blocking, the sepharose was washed using 3x medium volumes of $0.1 \mathrm{M}$ Tris- $\mathrm{HCl}$, $\mathrm{pH} 8-9$, 
followed by a wash with $0.1 \mathrm{M}$ acetate, $0.5 \mathrm{M} \mathrm{NaCl}, \mathrm{pH} 4-5$. These buffers were used alternately for a total of 8 column washes. The column was then transferred to SELEX buffer and stored at $4^{\circ} \mathrm{C}$ until use. This procedure was repeated to generate L-glycine and Tris-conjugated sepharose as controls.

\subsubsection{Ninhydrin test}

To confirm that L-serine had successfully coupled to the sepharose column, the ninhydrin test was used with the pre-coupling sample and post-coupling sample. A range of L-serine concentrations $(70-230 \mathrm{mM})$ were prepared in coupling buffer $\left(0.2 \mathrm{M} \mathrm{NaHCO}_{3}, 0.5 \mathrm{M} \mathrm{NaCl}, \mathrm{pH}\right.$ 8.3). A ninhydrin solution was prepared by adding $0.1 \mathrm{~g}$ of ninhydrin to $10 \mathrm{~mL}$ of glacial acetic acid. The ninhydrin solution was slightly heated until dissolved. Each concentration of L-serine, as well as the pre- and post-coupling solutions was mixed 1:1 with ninhydrin solution. Coupling buffer mixed with ninhydrin was used as a control. The solutions were left at room temperature 5-10 minutes before examining their colour change.

\subsubsection{Testing Hcy10 affinity for L-serine}

\subsubsection{Examining Hcy10 binding to L-serine-conjugated sepharose beads}

Hcy 10 with a 5'fluorescein modification was diluted in PBS buffer (10 mM Na2 $\mathrm{HPO}_{4}$, $1.8 \mathrm{mM} \mathrm{KH}_{2} \mathrm{PO}_{4}, 5 \mathrm{mM} \mathrm{MgCl}_{2}, 490 \mathrm{mM} \mathrm{NaCl}, \mathrm{pH}$ 7.4) over a range of concentrations from 20 $\mathrm{nM}$ to $1 \mu \mathrm{M}$. L-serine-coupled beads (see section 2.4.2) were diluted 1:1 in PBS buffer, and 20 $\mu \mathrm{L}$ of the bead slurry were added to $0.22 \mu \mathrm{m}$ Costar Spin-X cellulose acetate centrifuge filters for each aptamer concentration to be tested. The beads were washed by adding $100 \mu \mathrm{L}$ of PBS buffer and centrifuging at $13000 \times \mathrm{g}$. The DNA was heated at $90^{\circ} \mathrm{C}$ for 5 minutes, cooled at $4{ }^{\circ} \mathrm{C}$ for 10 minutes, and left at room temperature for 15 minutes. $100 \mu \mathrm{L}$ of each DNA concentration were added to L-serine-coupled beads, and placed on a shaker at room temperature for 45 
minutes. Unbound DNA was removed by centrifuging at $5000 \times \mathrm{g}$ for 1 minute, followed by 3 washes using $100 \mu \mathrm{L}$ of PBS buffer each. For elution of bound DNA, $100 \mu \mathrm{L}$ of $7 \mathrm{M}$ urea was added to the beads, vortexed, and incubated at $90^{\circ} \mathrm{C}$ for 10 minutes. The eluate was collected after centrifugation at $13000 \times \mathrm{g}$ for 1 minute. This was repeated for 3 elutions per test concentration. The fluorescence intensity of the elutions was measured using a Fluorolog Fluorescence Spectrophotometer (Horiba Jobin Yvon, USA) with an excitation wavelength of $490 \mathrm{~nm}$, emission wavelength of $520 \mathrm{~nm}$, and slit widths $5 \mathrm{~nm}$. The amount of DNA eluted at each concentration was calculated by comparing to a standard curve of fluorescence intensity at known concentrations.

\subsubsection{Examining Hcy10 binding to L-serine using microscale thermophoresis}

5'Fluorescein-modified Hcy10 was diluted to $10 \mu \mathrm{M}$ in PBS, and a $10 \mu \mathrm{L}$ sample prepared. A $1 \mathrm{~mL}$ L-serine sample was also prepared at $10 \mathrm{mM}$ in PBS, and sent to 2 bind in Germany for microscale thermophoresis, along with $35 \mathrm{~mL}$ of PBS. A range of L-serine concentrations $(7.6 \mathrm{nM}-250 \mu \mathrm{M}$ in buffer) was incubated with $20 \mathrm{nM}$ of 5'Fluoresceinmodified Hcy 10 and analyzed on a Monolith NT at $25^{\circ} \mathrm{C}$, with $15 \%$ LED power and $80 \%$ laser power.

\subsubsection{DNA Synthesis of each SELEX library}

Original Pool SELEX - The DNA pool for the original Pool SELEX had the following sequence, 5'-AGCAGCACAGAGGTCAGATG-N40-CCTATGCGTGCTACCGTGAA-3', where N represented a mixture of all 4 amidites with equal chance of being added, to create a pool of random sequences. For use in PCR, a forward primer 5'-6-FAMAGCAGCACAGAGGTCAGATG-3' was synthesized. A reverse primer 5'-poly-dA20-HEG- 
TTCACGGTAGCACGCATAGG was synthesized where HEG represented the hexaethylene glycol spacer phosphoramidite 18 .

Randomized Pool SELEX - The Hcy10 aptamer previously selected by M. McKeague was used as a template for the SELEX pool. The following sequence was synthesized: 5'-

ATACCAGCTTATTCAATTGTGGAAAGCCGAATGTGATTAGGGACCAGTGGAGAA GTAGTACGGACTGACCTCGCGTGTAAGATAGTAAGTGCAATCT-3', with the region shown in bold having a $15 \%$ randomization. For this region of the synthesis, $15 \%$ of the volume of each amidite contained an equimolar mixture of the three other amidites. A forward primer 5'6-FAM-ATACCAGCTTATTCAATT-3' was synthesized, and reverse primer 5'-dA20-HEGAGATTGCACTTACTATCT was synthesized.

Redirected Pool SELEX - For this SELEX pool, the last two rounds of SELEX completed by M. McKeague for L-homocysteine (rounds 7 and 8) were combined to create the starting pool for this SELEX. ${ }^{272}$ The primers synthesized for the randomized pool were also used for this SELEX.

After DNA synthesis, the CPG beads were transferred from the synthesis columns to microfuge tubes using needles, and $1 \mathrm{~mL}$ of $28 \%$ ammonium hydroxide solution was added. The beads were vortexed, vented, and heated at $55^{\circ} \mathrm{C}$ for 24 hours. After cooling to room temperature, the beads were centrifuged at $13000 \times \mathrm{g}$ for 5 minutes, and the supernatant collected. The beads were washed with $1 \mathrm{~mL}$ of $\mathrm{diH}_{2} \mathrm{O}$, centrifuged again, and the supernatant collected. The collected DNA solutions were dried down on a Savant AE2010 SpeedVac.

\subsubsection{Purification of DNA by denaturing PAGE}

The synthesized DNA strands were purified using $12 \%$ denaturing PAGE gels. The gel solution was composed of $31.5 \mathrm{~g}$ urea, $23.5 \mathrm{~mL}$ acrylamide (acrylamide/bis-acrylamide $40 \%$ 
solution), $15 \mathrm{~mL} 5 \times$ TBE buffer (45 mM Tris, $45 \mathrm{mM}$ boric acid, $1 \mathrm{mM}$ EDTA), and $14 \mathrm{~mL}$ deionized water for 2 gels. This solution was heated to $37^{\circ} \mathrm{C}$ with magnetic stirring, and filtered through Whatman No. 1 filter paper. Once cooled to room temperature, $450 \mu \mathrm{L}$ of $10 \%$ ammonium persulfate were added, and $35 \mu \mathrm{L}$ of N,N,N',N'-tetramethylethylenediamine (TEMED). The solution was quickly poured between pre-assembled glass plates, and a plastic comb was added to form the loading well. The gels were left to polymerize at room temperature for approximately 30 minutes. After polymerization, the plastic combs were removed, and the wells rinsed with $\mathrm{diH}_{2} \mathrm{O}$. The glass plates were transferred to a SE 600 Chroma Standard Dual gel electrophoresis unit (Hoefer). The gels were pre-run at a constant current of $30 \mathrm{~mA}(\sim 300$ volts) for 20 minutes. The DNA to be purified was resuspended in $\mathrm{diH}_{2} \mathrm{O}$, mixed 1:1 with formamide, and heated to $90^{\circ} \mathrm{C}$ prior to loading. The gels were run at a constant current of 30 $\mathrm{mA}$ (or $\sim 300$ volts) until an 18-base fluorescently tagged marker moved $3 / 4$ through the gel. The gels were imaged using an Alpha Innotech AlphaImager EC instrument, and the bands of interest excised from the gels. Each band was broken into pieces, and $\mathrm{diH}_{2} \mathrm{O}$ was added to extract the DNA from the gel pieces over 24 hours on a $37^{\circ} \mathrm{C}$ shaking incubator. Following this incubation, the gel slurry was filtered through $0.22 \mu \mathrm{m}$ cellulose acetate syringe filters to remove gel fragments. The DNA solution was frozen in liquid nitrogen and dried on a Labconco freezone lyophilizer. To remove salts, the DNA was resuspended in a minimum amount of $\operatorname{diH}_{2} \mathrm{O}$, and filtered using $3 \mathrm{kDa}$ cutoff Amicon Ultra centrifugal filter columns. The DNA was then quantified at $260 \mathrm{~nm}$ using a Varian Cary 300 Bio UV-Vis spectrophotometer, and the synthesis was confirmed using ESI/LC/MS. 


\subsubsection{SELEX methods and Conditions for each pool}

SELEX for L-serine was carried out using three separate starting pools: Original pool, Randomized pool, and Redirected pool. The general procedure is illustrated in Figure 2.3, and the specific conditions for each round are outlined in Table 2.2. For all selections, the negative column refers to sepharose beads coupled to Tris rather than the target, while the positive column refers to sepharose beads coupled to L-serine. Before each round of selection, the DNA pool in SELEX buffer (50 mM Tris- $\mathrm{HCl}, 500 \mathrm{mM} \mathrm{NaCl}, 5 \mathrm{mM} \mathrm{MgCl}_{2}, \mathrm{pH} 7.4$ ) was denatured at $90^{\circ} \mathrm{C}$ for 5 minutes, cooled at $4^{\circ} \mathrm{C}$ for 10 minutes, and brought to room temperature for 15 minutes. A $5 \mu \mathrm{L}$ sample of the DNA starting pool was set aside for fluorescence analysis. The column beads were placed in $0.22 \mu \mathrm{m}$ Costar Spin-X cellulose acetate centrifuge filters and washed 3 times with SELEX buffer prior to beginning. The starting DNA library was incubated with the negative column for 30 minutes shaking at room temperature in SELEX buffer. The beads were centrifuged at $14000 \times \mathrm{g}$ for 1 minute. The DNA solution that passed through the negative column was added to the positive column and incubated for 1 hour shaking at room temperature. The column was centrifuged at $5000 \times \mathrm{g}$ for 1 minute, collecting the flow-through. Four additional washes using SELEX buffer were also collected. The fourth wash was centrifuged at $14000 \times \mathrm{g}$ for 1 minute. DNA was eluted from the column by incubating the column with 100 mM L-serine for 10 minutes and centrifuging at $14000 \times \mathrm{g}$ to collect filtrate. This was repeated for 5 elutions. An additional 5 elutions were collected using $7 \mathrm{M}$ urea at $90^{\circ} \mathrm{C}$ to ensure most DNA was removed from the column. In the penultimate selection round for each SELEX experiment, counter selections against L-glycine and L-threonine were performed. Prior to eluting desired sequences using L-serine, an elution was performed using a solution of L-glycine and L-threonine (100 $\mathrm{mM}$ each), followed by one wash with SELEX buffer. The elution and 
wash of this counter selection were kept for high-throughput sequencing. The target incubation step in the final round of each SELEX experiment was performed using a 1:1 mixture of soil solution (obtained from sandy loam soil, uncropped for 15 years) and SELEX buffer. See Table 2.2 for a summary of experimental details.

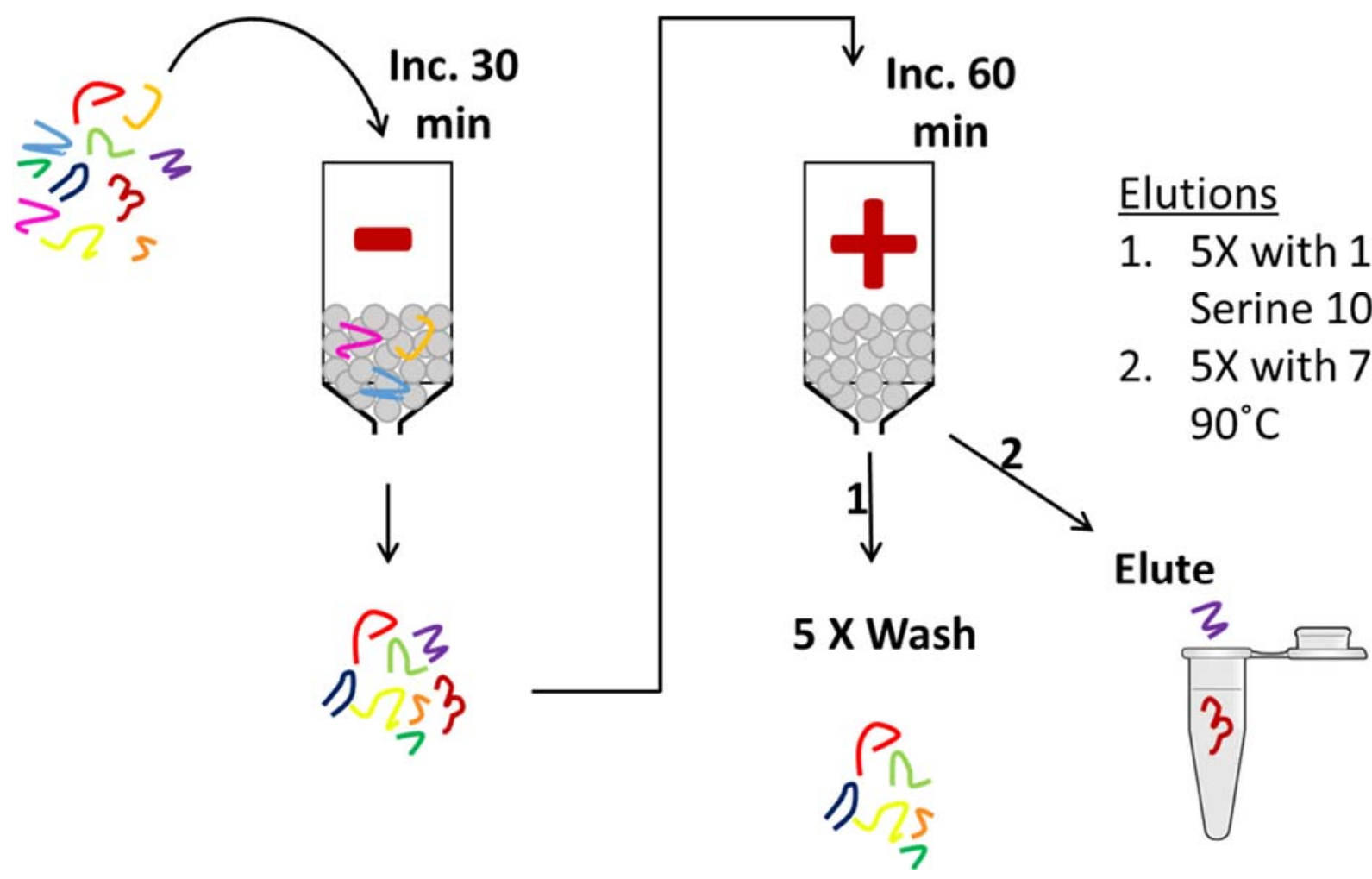

Figure 2.3: Overview of SELEX experimental procedure. A DNA library was added to Tris-conjugated sepharose for a "negative" selection (-). Sequences which did not bind sepharose were added to the $L$-serine-conjugated sepharose for a "positive" selection (+). L-serine-binding sequences were eluted with concentrated target, followed by urea.

The fluorescence of the elutions as well as the starting pool was measured using a Fluorolog Fluorescence Spectrophotometer (Horiba Jobin Yvon, USA) with an excitation wavelength of $490 \mathrm{~nm}$, emission wavelength of $520 \mathrm{~nm}$, and slit widths of $5 \mathrm{~nm}$. The amount of DNA bound to the columns as a measure of round-to-round enrichment could be followed by 
calculating the \% DNA bound as follows, where " $f$ elution" is the sum of the fluorescent intensities of all elutions, and " $f$ pool" is the total fluorescent intensity of the starting pool of the SELEX round.

$$
\% \text { DNA Bound }=\frac{f \text { elution }}{f \text { pool }} \times 100 \%
$$

The elutions were desalted using $3 \mathrm{kDa}$ cutoff Amicon Ultra centrifugal filter columns before being amplified using the polymerase chain reaction (PCR). 
Table 2.2: Experimental conditions in each SELEX round for the Original SELEX, Randomized SELEX, and the Redirected SELEX experiments.

\begin{tabular}{|c|c|c|c|}
\hline $\begin{array}{l}\text { SELEX } \\
\text { Round }\end{array}$ & Original SELEX & Randomized SELEX & Redirected SELEX \\
\hline 1 & $\begin{array}{l}5 \text { nmol DNA } \\
500 \mu \mathrm{L} \text { bead slurry } \\
1 \text { hour incubation time } \\
10 \text { Elutions }(500 \mu \mathrm{L}) \text { with } 10 \mathrm{mM} \mathrm{L}- \\
\text { serine } \\
2 \text { Elutions with } 7 \mathrm{M} \text { Urea }\end{array}$ & $\begin{array}{l}1 \text { nmol DNA } \\
100 \mu \mathrm{L} \text { bead slurry } \\
1 \text { hour incubation time } \\
5 \text { Elutions }(100 \mu \mathrm{L}) \text { with } 100 \mathrm{mM} \mathrm{L}- \\
\text { serine } \\
5 \text { Elutions with } 7 \mathrm{M} \text { urea }\end{array}$ & $\begin{array}{l}1 \text { nmol DNA } \\
250 \mu \mathrm{L} \text { bead slurry } \\
1 \text { hour incubation time } \\
5 \text { Elutions }(100 \mu \mathrm{L}) \text { with } 100 \mathrm{mM} \\
\text { L-serine } \\
5 \text { Elutions with } 7 \mathrm{M} \text { urea }\end{array}$ \\
\hline 2 & $\begin{array}{l}1 \text { nmol DNA } \\
500 \mu \mathrm{L} \text { bead slurry } \\
1 \text { hour incubation time } \\
10 \text { Elutions }(500 \mu \mathrm{L}) \text { with } 10 \mathrm{mM} \mathrm{L}- \\
\text { serine } \\
2 \text { Elutions with } 7 \mathrm{M} \text { Urea }\end{array}$ & $\begin{array}{l}200 \text { pmol DNA } \\
100 \mu \mathrm{L} \text { bead slurry } \\
1 \text { hour incubation time } \\
5 \text { Elutions }(100 \mu \mathrm{L}) \text { with } 100 \mathrm{mM} \mathrm{L}- \\
\text { serine } \\
5 \text { Elutions with } 7 \mathrm{M} \text { urea }\end{array}$ & $\begin{array}{l}200 \text { pmol DNA } \\
250 \mu \mathrm{L} \text { bead slurry } \\
1 \text { hour incubation time } \\
5 \text { Elutions }(100 \mu \mathrm{L}) \text { with } 100 \mathrm{mM} \\
\text { L-serine } \\
5 \text { Elutions with } 7 \mathrm{M} \text { urea }\end{array}$ \\
\hline 3 & $\begin{array}{l}200 \text { pmol DNA } \\
500 \mu \mathrm{L} \text { bead slurry } \\
1 \text { hour incubation time } \\
10 \text { Elutions }(500 \mu \mathrm{L}) \text { with } 10 \mathrm{mM} \mathrm{L}- \\
\text { serine } \\
2 \text { Elutions with } 7 \mathrm{M} \text { Urea }\end{array}$ & $\begin{array}{l}200 \text { pmol DNA ( } 125 \text { pmol L-serine- } \\
\text { eluted, } 75 \text { pmol urea-eluted) } \\
100 \mu \mathrm{L} \text { bead slurry } \\
1 \text { hour incubation time } \\
5 \text { Elutions }(100 \mu \mathrm{L}) \text { with } 100 \mathrm{mM} \mathrm{L-} \\
\text { serine } \\
5 \text { Elutions with } 7 \mathrm{M} \text { urea }\end{array}$ & $\begin{array}{l}200 \text { pmol DNA ( } 125 \text { pmol L-serine- } \\
\text { eluted, } 75 \text { pmol urea-eluted) } \\
250 \mu \mathrm{L} \text { bead slurry } \\
1 \text { hour incubation time } \\
5 \text { Elutions }(100 \mu \mathrm{L}) \text { with } 100 \mathrm{mM} \\
\text { L-serine } \\
5 \text { Elutions with } 7 \mathrm{M} \text { urea }\end{array}$ \\
\hline 4 & $\begin{array}{l}200 \text { pmol DNA } \\
500 \mu \mathrm{L} \text { bead slurry } \\
30 \text { min incubation time } \\
10 \text { Elutions }(500 \mu \mathrm{L}) \text { with } 10 \mathrm{mM} \mathrm{L-} \\
\text { serine } \\
2 \text { Elutions with } 7 \mathrm{M} \text { Urea }\end{array}$ & $\begin{array}{l}500 \text { pmol DNA ( } 300 \text { pmol L-serine- } \\
\text { eluted, } 200 \text { pmol urea-eluted) } \\
100 x \text { Diluted beads } \\
30 \text { minute incubation time } \\
5 \text { Elutions }(100 \mu \mathrm{L}) \text { with } 100 \mathrm{mM} \mathrm{L-} \\
\text { serine } \\
5 \text { Elutions with } 7 \mathrm{M} \text { urea }\end{array}$ & $\begin{array}{l}200 \text { pmol DNA ( } 125 \text { pmol L-serine- } \\
\text { eluted, } 75 \text { pmol urea-eluted) } \\
100 x \text { Diluted beads } \\
\text { Counter selections with } 100 \mathrm{mM} \mathrm{L-} \\
\text { Glycine and L-Threonine } \\
20 \text { min incubation time } \\
5 \text { Elutions }(100 \mu \mathrm{L}) \text { with } 100 \mathrm{mM} \\
\text { L-serine } \\
5 \text { Elutions with } 7 \mathrm{M} \text { urea }\end{array}$ \\
\hline
\end{tabular}




\begin{tabular}{|c|c|c|c|}
\hline 5 & $\begin{array}{l}200 \text { pmol DNA } \\
500 \mu \mathrm{L} \text { bead slurry } \\
30 \text { min incubation time } \\
10 \text { Elutions }(500 \mu \mathrm{L}) \text { with } 100 \mathrm{mM} \mathrm{L-} \\
\text { serine } \\
2 \text { Elutions with } 7 \mathrm{M} \text { Urea }\end{array}$ & $\begin{array}{l}500 \text { pmol DNA ( } 125 \text { pmol L-serine- } \\
\text { eluted, } 75 \text { pmol urea-eluted) } \\
100 x \text { Diluted beads } \\
30 \text { minute incubation time } \\
5 \text { Elutions }(100 \mu \mathrm{L}) \text { with } 100 \mathrm{mM} \mathrm{L-} \\
\text { serine } \\
5 \text { Elutions with } 7 \mathrm{M} \text { urea }\end{array}$ & $\begin{array}{l}200 \text { pmol DNA ( } 125 \text { pmol L-serine- } \\
\text { eluted, } 75 \text { pmol urea-eluted) } \\
100 x \text { Diluted beads } \\
20 \text { min incubation time in } 1: 1 \text { Soil } \\
\text { Extract: SELEX buffer } \\
5 \text { Elutions }(100 \mu \mathrm{L}) \text { with } 100 \mathrm{mM} \\
\text { L-serine } \\
5 \text { Elutions with } 7 \mathrm{M} \text { urea }\end{array}$ \\
\hline 6 & $\begin{array}{l}200 \text { pmol DNA } \\
500 \mu \mathrm{L} \text { bead slurry } \\
30 \text { min incubation time } \\
5 \text { Elutions }(500 \mu \mathrm{L}) \text { with } 100 \mathrm{mM} \mathrm{L-} \\
\text { serine } \\
5 \text { Elutions with } 7 \mathrm{M} \text { Urea }\end{array}$ & $\begin{array}{l}500 \text { pmol DNA ( } 125 \text { pmol L-serine- } \\
\text { eluted, } 75 \text { pmol urea-eluted) } \\
100 x \text { Diluted beads } \\
30 \text { minute incubation time } \\
5 \text { Elutions }(100 \mu \mathrm{L}) \text { with } 100 \mathrm{mM} \mathrm{L-} \\
\text { serine } \\
5 \text { Elutions with } 7 \mathrm{M} \text { urea }\end{array}$ & \\
\hline 7 & $\begin{array}{l}200 \text { pmol DNA } \\
500 \mu \mathrm{L} \text { bead slurry } \\
20 \text { min incubation time } \\
5 \text { Elutions }(500 \mu \mathrm{L}) \text { with } 100 \mathrm{mM} \mathrm{L-} \\
\text { serine } \\
5 \text { Elutions with } 7 \mathrm{M} \text { Urea }\end{array}$ & $\begin{array}{l}500 \text { pmol DNA ( } 125 \text { pmol L-serine- } \\
\text { eluted, } 75 \text { pmol urea-eluted) } \\
100 x \text { Diluted beads } \\
\text { Counter selections with } 100 \mathrm{mM} \mathrm{L-} \\
\text { Glycine and L-Threonine } \\
20 \text { minute incubation time } \\
5 \text { Elutions }(100 \mu \mathrm{L}) \text { with } 100 \mathrm{mM} \mathrm{L-} \\
\text { serine } \\
5 \text { Elutions with } 7 \mathrm{M} \text { urea }\end{array}$ & \\
\hline 8 & $\begin{array}{l}200 \text { pmol DNA ( } 125 \text { pmol L-serine- } \\
\text { eluted, } 75 \text { pmol urea-eluted) } \\
500 \mu \mathrm{L} \text { bead slurry } \\
20 \text { min incubation time } \\
5 \text { Elutions }(500 \mu \mathrm{L}) \text { with } 100 \mathrm{mM} \mathrm{L-} \\
\text { serine } \\
5 \text { Elutions with } 7 \mathrm{M} \text { urea }\end{array}$ & $\begin{array}{l}500 \text { pmol DNA ( } 125 \text { pmol L-serine- } \\
\text { eluted, } 75 \text { pmol urea-eluted) } \\
100 x \text { Diluted beads } \\
20 \text { min incubation time in } 1: 1 \text { Soil } \\
\text { Extract: SELEX buffer } \\
5 \text { Elutions }(100 \mu \mathrm{L}) \text { with } 100 \mathrm{mM} \mathrm{L-} \\
\text { serine } \\
5 \text { Elutions with } 7 \mathrm{M} \text { urea }\end{array}$ & \\
\hline
\end{tabular}




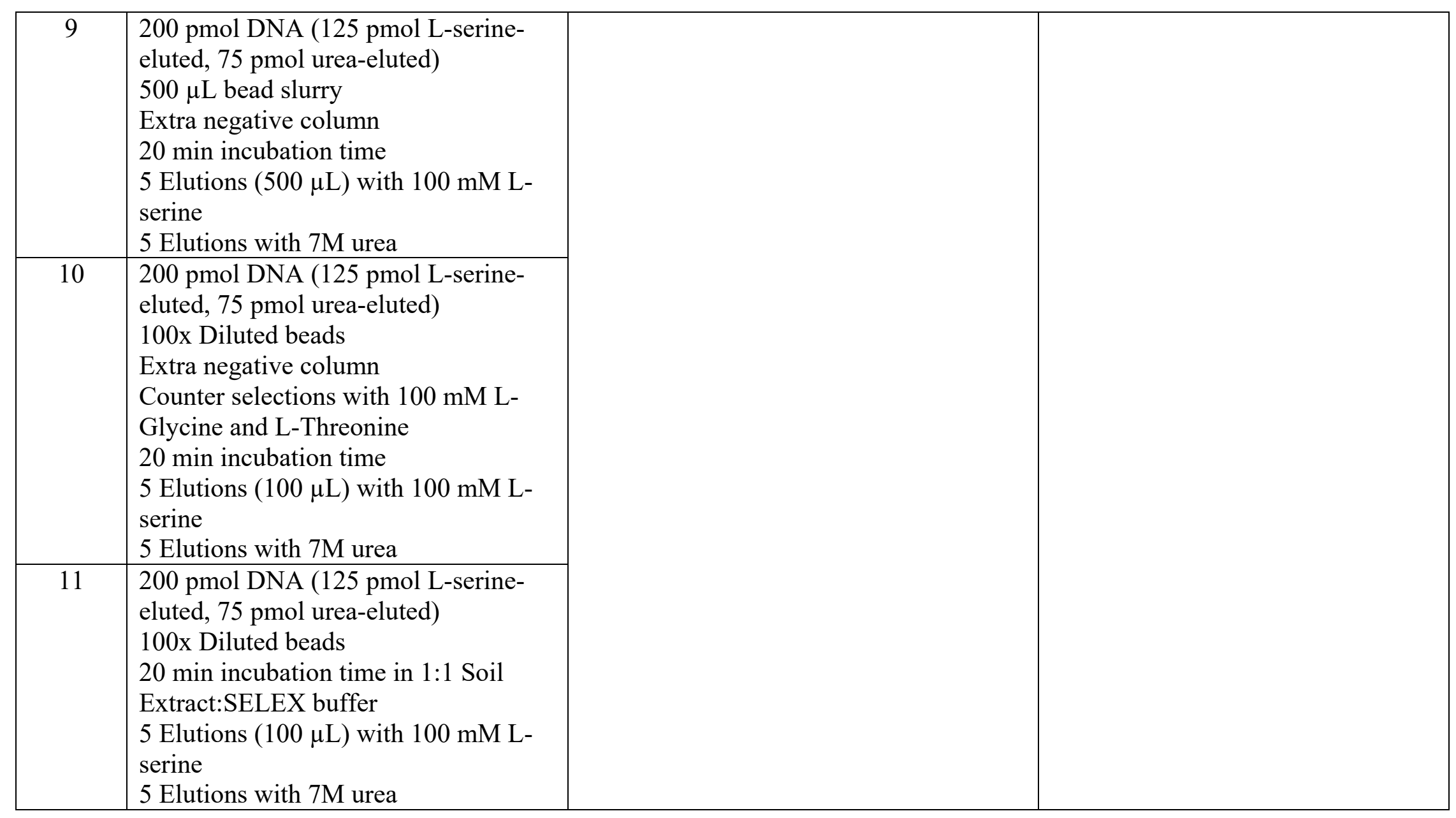

Counter selections: $100 \mu \mathrm{L}$ solution of L-glycine and L-threonine (100 mM each) were added to beads, incubated 10 minutes, centrifuged, and washed once with SELEX buffer before conducting elutions with L-serine. 


\subsubsection{Polymerase Chain Reaction (PCR) to amplify SELEX rounds}

PCR reactions were prepared to a volume of $100 \mu \mathrm{L}$ by combining: $50 \mu \mathrm{L}$ FluMag buffer $(0.2 \mathrm{M} \text { Tris- } \mathrm{HCl} \mathrm{pH} 9,0.1 \mathrm{M} \mathrm{KCl}, 2 \% \text { triton } \mathrm{X}-100)^{273}, 37 \mu \mathrm{L} \mathrm{diH}_{2} \mathrm{O}, 8 \mu \mathrm{L} 25 \mathrm{mM} \mathrm{MgCl} 2,2 \mu \mathrm{L}$ of dNTP mix, $0.5 \mu \mathrm{L}$ of fluorescently labelled forward primer, $0.5 \mu \mathrm{L}$ of polyA reverse primer, 1 $\mu \mathrm{L}$ of Taq DNA polymerase, and $1 \mu \mathrm{L}$ of the DNA library to be amplified. Positive and negative control reactions were run by substituting $1 \mu \mathrm{L}$ of control pool, or $\mathrm{diH}_{2} \mathrm{O}$ in place of the SELEX pool, respectively. PCR was run on a Mastercycler epgradient (Eppendorf, Hauppauge, NY, USA). The PCR conditions for the original pool SELEX were: denaturation at $94^{\circ} \mathrm{C}$ for 5 minutes; 25 cycles of (melting at $94^{\circ} \mathrm{C}$ for 30 seconds, annealing at $54^{\circ} \mathrm{C}$ for 30 seconds, extension at $74^{\circ} \mathrm{C}$ for 20 seconds); final extension at $72^{\circ} \mathrm{C}$ for 5 minutes. For the randomized pool and redirected pool SELEX, the PCR conditions were: denaturation at $94^{\circ} \mathrm{C}$ for 10 minutes; 25 cycles of (melting at $94^{\circ} \mathrm{C}$ for 1 minute, annealing at $47^{\circ} \mathrm{C}$ for 1 minute, extension at $72^{\circ} \mathrm{C}$ for 1 minute); final extension at $72^{\circ} \mathrm{C}$ for 10 minutes. The PCR conditions were optimized for each primer set.

After PCR, the reactions were dried down on a Savant AE2010 SpeedVac. They were resuspended in a minimum volume of $\mathrm{diH}_{2} \mathrm{O}$, mixed 1:1 with formamide, and run on $12 \%$ denaturing PAGE gels as described in section 2.4.6, cutting out the fluorescent band. This DNA was eluted from the PAGE gels, and desalted as previously described. The DNA was quantified at $260 \mathrm{~nm}$ using a Varian Cary 300 Bio UV-Vis spectrophotometer, and used as the starting pool for the subsequent SELEX round.

\subsubsection{Preparing DNA libraries for sequencing}

The Illumina adapter sequences were designed with help from Genome Quebec, and were ordered from IDT, PAGE-purified with mass confirmation by mass spectrometry, shown in 
Table 7.1. The adapters were added to DNA libraries by PCR amplification. For the original pool SELEX, a master mix was made containing: $350 \mu$ L FluMag buffer $(0.2 \mathrm{M}$ Tris-HCl pH 9, $0.1 \mathrm{M}$ $\mathrm{KCl}, 2 \%$ triton $\mathrm{X}-100), 260 \mu \mathrm{L} \mathrm{diH} 2 \mathrm{O}, 56 \mu \mathrm{L} \mathrm{MgCl}_{2}, 14 \mu \mathrm{L}$ dNTPs, $10.5 \mu \mathrm{L}$ forward primer, $10.5 \mu \mathrm{L}$ reverse primer, and $7 \mu \mathrm{L}$ Taq. The master mix was mixed well and $95 \mu \mathrm{L}$ was removed for a PCR control. To the rest of the master mix, template was added $(6 \mu \mathrm{L}$ of the original pool, or $36 \mu \mathrm{L}$ of a SELEX round library) and divided into 6 reactions. The PCR conditions were as described in section 2.4.8, using a gradient annealing temperature from $54.1^{\circ} \mathrm{C}$ to $56.4^{\circ} \mathrm{C}$ and a total of 20 cycles. For randomized SELEX, the same master mix was used, with $20 \mu \mathrm{L}$ of template added ( $6 \mu \mathrm{L}$ of the round 1 pool) and divided into 6 reactions. The cycle used was as described in section 2.4 .8 with a gradient of annealing temperatures from $49-59^{\circ} \mathrm{C}$. For the redirected SELEX, a master mix was made containing: $350 \mu \mathrm{L}$ FluMag buffer, $266 \mu \mathrm{L} \mathrm{diH}_{2} \mathrm{O}$, $56 \mu \mathrm{L} \mathrm{MgCl}_{2}, 14 \mu \mathrm{L}$ dNTPs, $15 \mu \mathrm{L}$ forward primer, $15 \mu \mathrm{L}$ reverse primer, and $7 \mu \mathrm{L}$ Taq. After removing $95 \mu \mathrm{L}$ for a PCR control, 20-28 $\mu \mathrm{L}$ of each template was added, and divided in 6 reactions. The cycle used was as described in section 2.4 .8 with gradient annealing temperatures from $49-59^{\circ} \mathrm{C}$ for 20 cycles.

After each PCR, like reactions were combined and dried down on a Savant AE2010 SpeedVac. The DNA libraries now with Illumina adapters attached were purified and extracted from denaturing PAGE gels as previously described, using 8\% gels to accommodate the longer sequence length. The $8 \%$ gels were composed of $8 \mathrm{~mL}$ acrylamide (acrylamide/bis-acrylamide $40 \%$ solution), $15.25 \mathrm{~g}$ Urea, $7.5 \mathrm{~mL} 5 \times \mathrm{TBE}$ buffer, and $21.5 \mathrm{~mL} \mathrm{diH} 2 \mathrm{O}$ for one gel. After purification and extraction from the $8 \%$ denaturing PAGE gels, each DNA library was quantified using a Nanodrop spectrophotometer. Eight DNA libraries were sent in each sequencing reaction, and the SELEX libraries that were sent and the adapters used are shown below. 
Table 2.3: SELEX pools examined using Illumina high throughput sequencing

Sequencing reaction 1:

Randomized SELEX

\begin{tabular}{|c|l|}
\hline Adapters & \multicolumn{1}{c|}{ SELEX Pool } \\
\hline F1 + R1 & Randomized Hcy10 starting library (Round 0) \\
\hline F1 + R2 & SELEX pool binding L-serine from round 7 \\
\hline F2 + R3 & SELEX pool binding L-serine from round 8 (selection in soil solution) \\
\hline F2 + R4 & $\begin{array}{l}\text { SELEX pool binding sepharose (Round 8), combined with counter } \\
\text { selections for L-glycine and L-threonine (Round 7) - CONTROL }\end{array}$ \\
\hline
\end{tabular}

\section{Original SELEX}

\begin{tabular}{|c|l|}
\hline Adapters & \multicolumn{1}{c|}{ SELEX Pool } \\
\hline F3 + R5 & Original Pool starting library (Round 0) \\
\hline F3 + R6 & SELEX pool binding L-serine from round 10 \\
\hline F4 + R8 & SELEX pool binding L-serine from round 11 (selection in soil solution) \\
\hline F4 + R7 & $\begin{array}{l}\text { SELEX pool binding sepharose (Round 11), combined with counter } \\
\text { selections for L-glycine and L-threonine (Round 10) - CONTROL }\end{array}$ \\
\hline
\end{tabular}

\section{Sequencing reaction 2:}

Redirected SELEX

\begin{tabular}{|c|l|}
\hline Adapters & \multicolumn{1}{c|}{ SELEX Pool } \\
\hline F1 + R1 & Starting library (last 2 rounds of L-homocysteine selection) \\
\hline F1 + R2 & SELEX pool binding L-serine from round 4 \\
\hline F2 + R3 & SELEX pool binding L-serine from round 5 (selection in soil solution) \\
\hline F2 + R4 & $\begin{array}{l}\text { SELEX pool binding sepharose (Round 5), combined with counter } \\
\text { selections for L-glycine and L-threonine (Round 4) - CONTROL }\end{array}$ \\
\hline
\end{tabular}




\subsubsection{High throughput sequencing (HTS)}

All DNA libraries were diluted to $5 \mathrm{ng} / \mu \mathrm{L}$, and $40 \mathrm{ng}$ of each library was added to a microwell plate ( 8 libraries were present per sequencing reaction, using adapters R1-R8). The Original SELEX libraries, and the Randomized SELEX libraries were sent in one reaction, while the Redirected SELEX was sent separately. Four DNA pools were sent for each SELEX experiment (listed in Table 2.3). The sequencing reactions were sent to Genome Quebec, who performed HiSeq 2000 PE100 sequencing, (http://www.genomequebec.com/en/home.html). Genome Quebec provided sequencing data in the form of .fastq files to be analyzed with highthroughput sequencing software.

\subsubsection{Analysis using AptaCluster}

The analysis of sequencing files was done using AptaCluster software with the help of J. Hoinka from National Institutes of Health (NIH). A configuration file was made for each SELEX experiment, outlining the selection-specific parameters such as: template length, primer sequences, and the number of selection rounds. The quality control parameters, locality sensitive hashing options, and the cluster relations settings were not changed from the program's default settings. For the randomized Hcy10 pool SELEX, the lsh_sequence_similarity parameter was reduced from the default 6 to 4 , as this library was expected to contain much more sequence similiarity than the other selection libraries. Though the experimental design of the sequencing experiment allowed multiple selection libraries to be sequenced at once, the configuration files for each selection experiment were run individually in AptaCluster, and the data was examined using AptaGUI. 


\subsection{Results and Discussion}

\subsubsection{Coupling L-serine to sepharose beads}

As L-serine is a small molecule, it had to be immobilized in order to perform selections. Two of the starting libraries used were modified from a previous selection for L-homocysteine, in which L-homocysteine was immobilized to a sepharose resin. ${ }^{272}$ To minimize non-specific binding, the resin was kept consistent, and L-serine was coupled to NHS-Sepharose Fast Flow resin (Sigma). In this coupling process, the sepharose contained an N-hydroxysuccinimide active ester which readily reacts with primary amines generating a stable amide bond, shown in Figure 2.4.

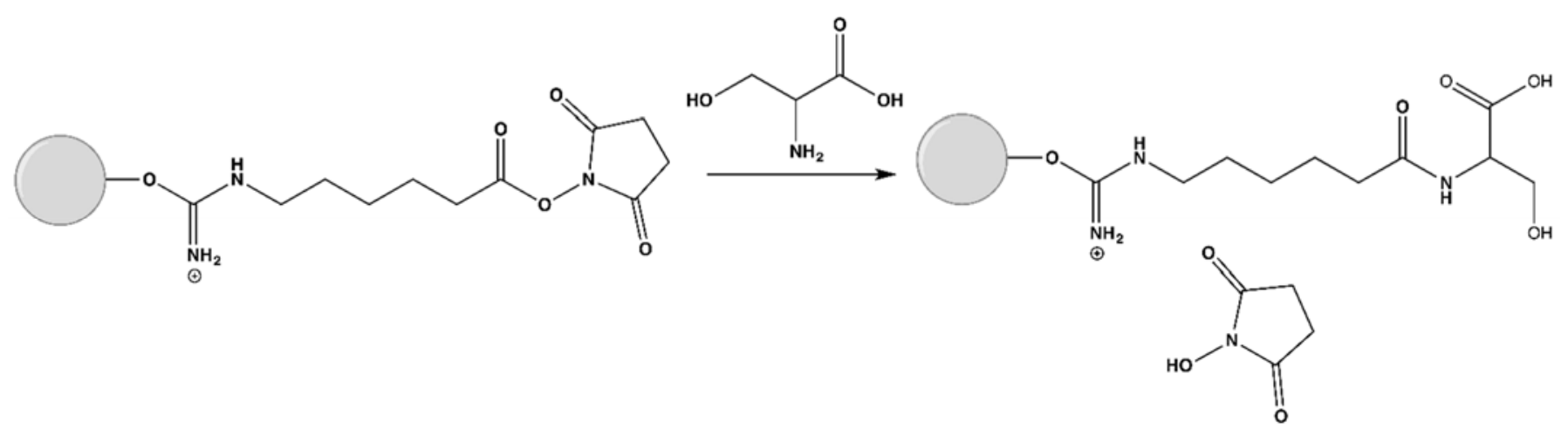

Figure 2.4: Coupling reaction of L-serine to NHS-sepharose 4 fast flow resin.

Target immobilization is often a hindrance to small molecule selections, as one of very few functional groups is removed from the binding reactions during selection. In this selection, the amine was chosen for immobilization strategically, in an effort to minimize the cross reactivity of a selected aptamer for other amino acids who share this amine group, such as Lthreonine and L-glycine. Using the amine group for coupling also allowed for simple determination of coupling efficiency through the colorimetric ninhydrin test. Determining the coupling efficiency of the target to the solid-support confirms the target is present prior to 
starting selections. Having an estimate of the target concentration present throughout the selection also allows the stringency of the selection to be more effectively adjusted. Ninhydrin hydrate reacts with amino groups to produce the coloured chromophore called Ruhemann's purple, which can be measured at $570 \mathrm{~nm} .{ }^{274}$ In order to ensure L-serine was successfully coupled to the sepharose resin, samples of pre-coupling and post-coupling solutions were reacted with ninhydrin and compared, shown in Figure 2.5.

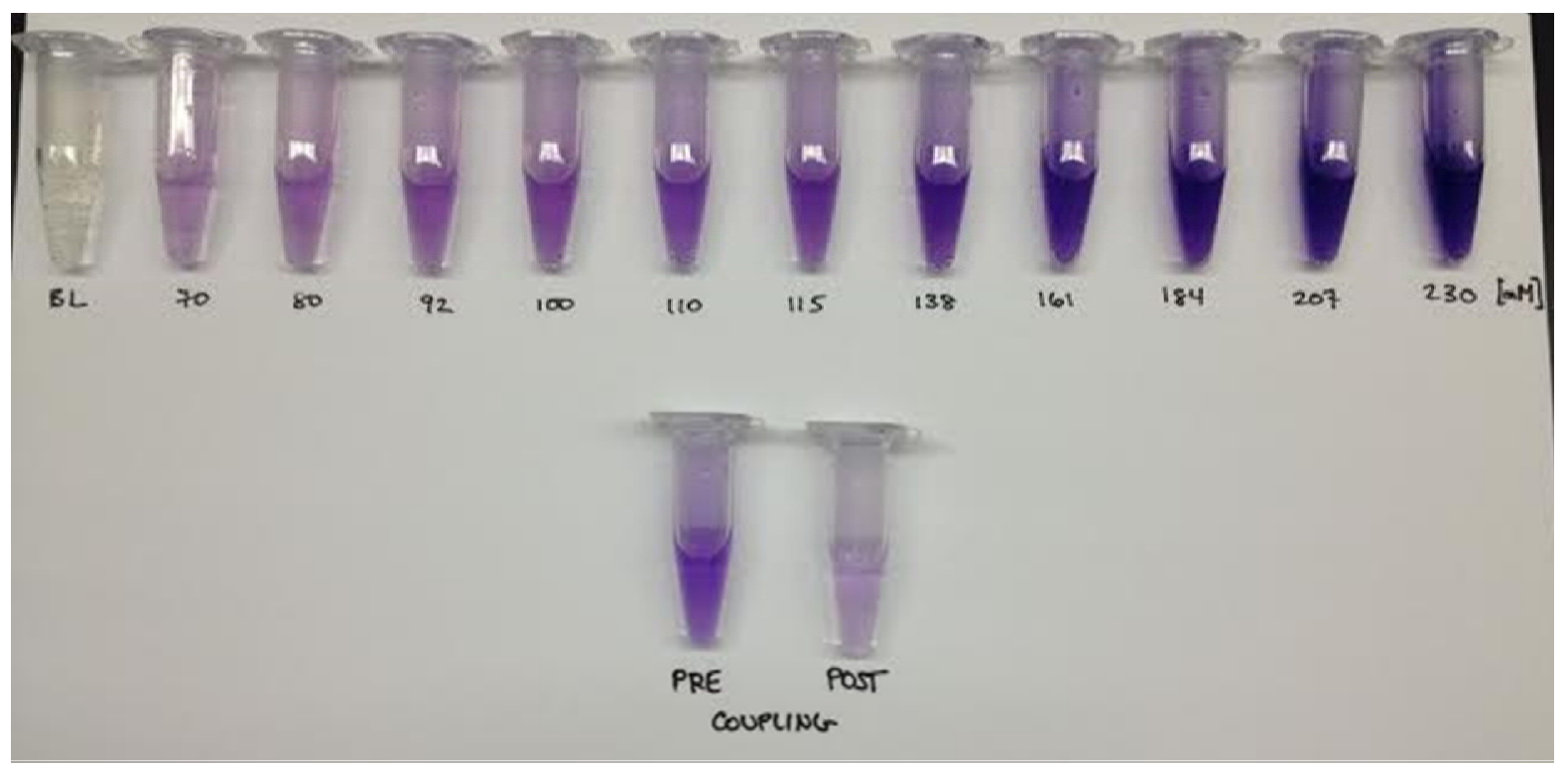

\section{Figure 2.5: Colorimetric ninhydrin test showing successful coupling of L-serine to the sepharose matrix.}

As can be seen in Figure 2.5, the deep purple of the pre-coupling solution indicated the presence of L-serine in solution. This deep purple colour had diminished considerably in the post-coupling solution, which indicated the L-serine had successfully coupled to the sepharose resin. A standard curve was made to estimate the amount of L-serine coupled to the sepharose matrix, from which it was determined a quantitative coupling efficiency to the beads, with 16-23 $\mu \mathrm{mol} / \mathrm{mL}$ ligand density. 


\subsubsection{Evaluation of Hcy10 affinity for L-serine using multiple methods}

A selection for the small molecule L-homocysteine had been previously completed in the DeRosa lab, resulting in two aptamers, Hcy8 and Hcy $10 .{ }^{272}$ These aptamers were previously screened for binding to similar small molecules L-cysteine, L-methionine, and L-serine, using sepharose and magnetic beads. The apparent dissociation constants of Hcy8 and Hcy10 to these small molecules are shown in Table 2.4.

Table 2.4: Preliminary affinity screening of L-homocysteine aptamers for small molecules L-cysteine, L-methionine, and L-serine, performed by M. McKeague.

\begin{tabular}{|l|c|c|}
\multicolumn{1}{|c|}{ Target } & Hcy8 & $\mathbf{K}_{\mathbf{d}}(\boldsymbol{\mu M})$ \\
\hline L-homocysteine sepharose & 0.8 & Hcy10 \\
\hline L-homocysteine magnetic beads & $0.6 \pm 0.3$ & 3.0 \\
\hline L-cysteine magnetic beads & 22 & $1.2 \pm 0.7$ \\
\hline L-methionine magnetic beads & 44 & 91 \\
\hline L-serine magnetic beads & $\mathbf{8}$ & $\mathbf{0 . 5}$ \\
\hline
\end{tabular}

Both Hcy8 and Hcy10 showed some binding to L-serine, with Hcy10 showing an apparent $\mathrm{K}_{\mathrm{d}}$ around $0.5 \mu \mathrm{M}$. As an L-serine-binding aptamer was desired, Hcy10 was evaluated as a possible L-serine-binding aptamer as it showed preference for L-serine over L-homocysteine in the preliminary binding tests shown in Table 2.4 .

To further characterize the binding of Hcy10 to L-serine, Hcy10 was synthesized and its binding to immobilized L-serine was examined using L-serine-conjugated sepharose beads, while microscale thermophoresis was used to evaluate the affinity of Hcy10 to L-serine in solution. A constant amount of L-serine-conjugated beads was incubated with a range of Hcy10 concentrations, non-binding DNA was washed away, and binding Hcy10 sequences were eluted from the L-serine-conjugated beads and quantified. If target-binding occurred, an increasing amount of Hcy10 was expected to be eluted from the beads, as the aptamer concentration 
increased until a plateau was reached. Figure 2.6 shows a binding isotherm obtained after incubating a range of Hcy10 concentrations with L-serine-conjugated sepharose beads.

Microscale thermophoresis evaluated aptamer-target binding based on small changes in molecular movement over a temperature gradient, and was used to determine the binding affinity of Hcy10 to L-serine in solution. For this test, the Hcy10 concentration was constant and was incubated with a range of L-serine concentrations in PBS buffer. Target-binding would cause a trend to be seen in the change of thermophoretic mobility of the DNA with increasing target concentration. The results obtained from microscale thermophoretic analysis of Hcy 10 with Lserine are shown in Figure 2.7.

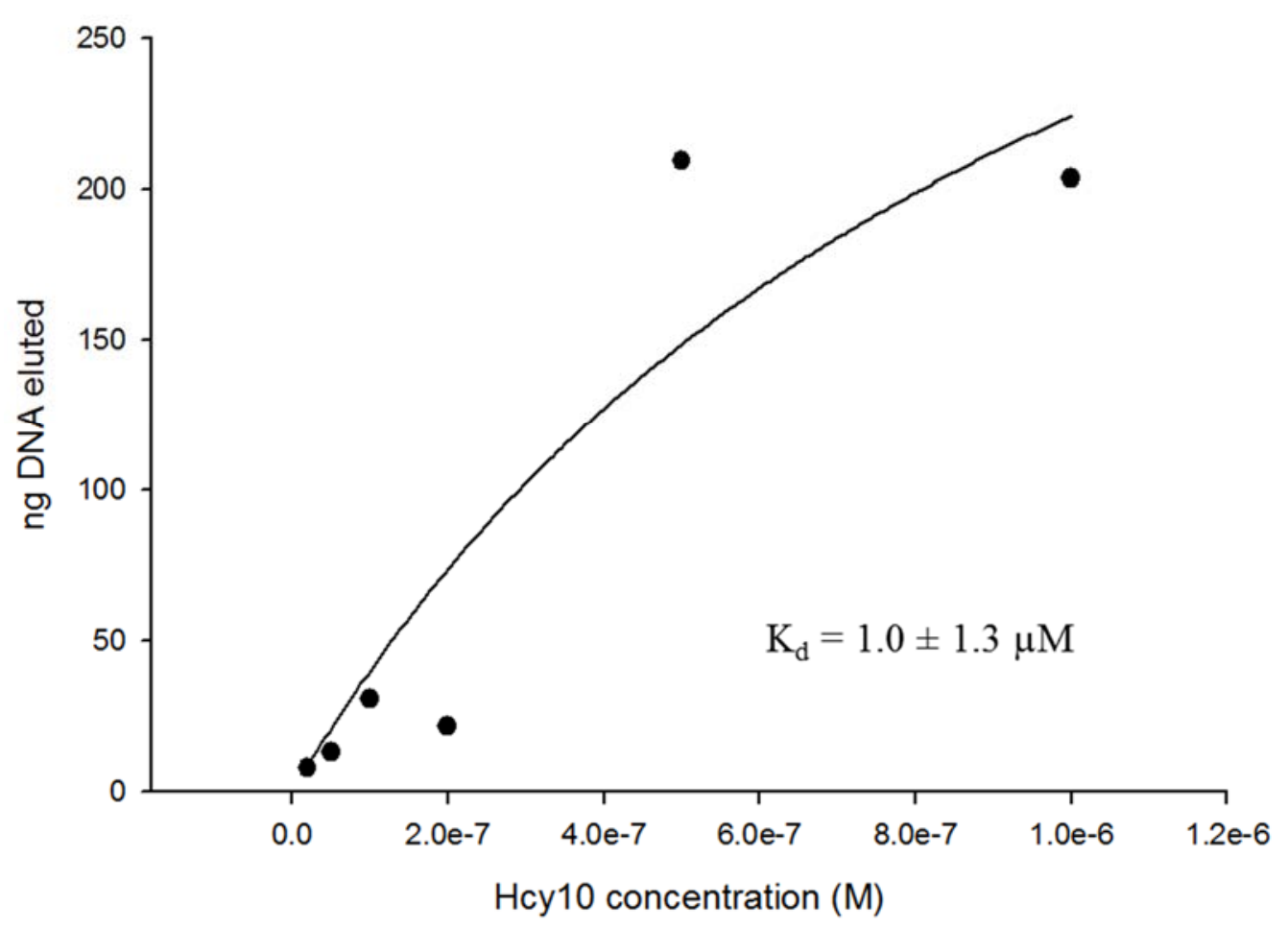

Figure 2.6: Binding isotherm showing Hcy10 binding L-serine-conjugated sepharose beads. A range of fluorescently modified Hcy10 concentrations $(20 \mathrm{nM}$ to $1 \mu \mathrm{M})$ was incubated with L-serine-coupled beads, and eluted with excess L-serine and $7 \mathrm{M}$ urea. Fluorescence spectroscopy was used to measure the Hcy10 eluted. This binding isotherm was generated using simple ligand-binding in SigmaPlot, and the error is on the fit. 


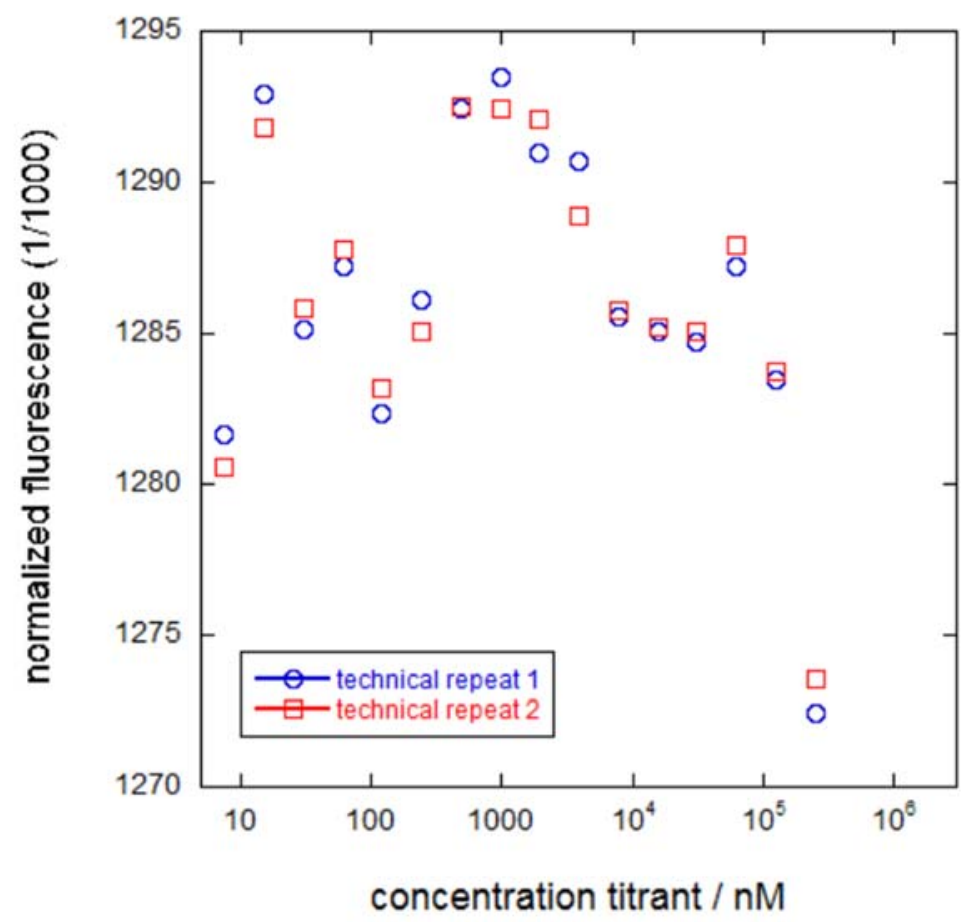

Figure 2.7: Binding of Hcy10 to L-serine in solution was examined using microscale thermophoresis. Fluorescently labeled-Hcy10 $(20 \mathrm{nM})$ was monitored after its incubation with L-serine (7.6 $\mathrm{nM}-250 \mu \mathrm{M}$ in buffer). No trend was observed, indicating no binding of Hcy10 to L-serine in PBS.

From the L-serine-sepharose binding test shown in Figure 2.6, an increasing amount of Hcy10 was eluted from the L-serine-conjugated sepharose beads, indicating L-serine-binding. From this isotherm, an apparent $\mathrm{K}_{\mathrm{d}}$ of $1.0 \pm 1.3 \mu \mathrm{M}$ was obtained which confirmed that Hcy10 showed some affinity for L-serine when it was immobilized to sepharose. Microscale thermophoresis examined the change in thermophoresis observed when Hcy10 was incubated with increasing L-serine concentrations. Figure 2.7 shows the result from this examination, and no trend was observed, which indicated that Hcy10 was unable to bind L-serine in solution. Since Hcy10 showed binding to immobilized L-serine but failed to bind L-serine in solution, it was possible that the solid support was involved in the binding interaction. An L-serine-binding aptamer was desired that was capable of binding L-serine in solution, as L-serine is released by 
crops. Hcy 10 would be unable to fulfill this role, as no binding to L-serine in solution was observed.

\subsubsection{SELEX Library Design}

In section 2.4.4, it was found that the L-homocysteine-binding aptamer, Hcy10, was able to bind immobilized L-serine, though unable to bind L-serine in solution. It was postulated that better L-serine-binding aptamers could exist within the L-homocysteine pool, or that the Hcy10 aptamer could be improved through base substitutions to gain more affinity for L-serine. The Lhomocysteine selection underwent five rounds of negative selection against sepharose beads, as well as three rounds of counter selections against L-cysteine, and L-methionine. ${ }^{272}$ Creating libraries from this selection could provide starting SELEX libraries with low affinity for sepharose, which would be useful in small-molecule selections where non-specific binding is a challenge. These libraries could also be more selective against similar amino acids L-cysteine and L-methionine as these amino acids were selected against. With this in mind, two SELEX libraries to find L-serine-binding aptamers were designed from this L-homocysteine selection.

The Hcy10 aptamer showed some affinity for immobilized-L-serine, but not for L-serine in solution. To try to improve the affinity, a SELEX library referred to as "Randomized SELEX" was designed using the Hcy10 aptamer as a template in which the 61-base random region was mutated by $15 \%$. This generated a library of mutations to be tested for L-serine-binding, with the goal of identifying beneficial mutations to increase binding affinity. This aptamer randomization strategy has been successful in generating L-arginine and L-tyrosine-binding aptamers, from Lcitrulline and dopamine-binding aptamers, repectively. ${ }^{158,165}$ A second library was created by combining the final two selection rounds from the L-homocysteine selection, referred to as "Redirected SELEX". Though L-serine was not the target for the L-homocysteine selection, 
Hcy10 was still able to bind L-serine. The goal of this redirected SELEX was to find any other sequences with affinity for L-serine in the L-homocysteine library, and enrich them through several selection rounds. Finally, a third SELEX library was created that had never been used for a selection, referred to as "Original SELEX". This traditional library was created to have no selective bias.

The template length used for the Randomized SELEX and the Redirected SELEX was 97 bases with a 61-base random region, which was on the long end of the typical range for a selection template. ${ }^{18}$ Though random regions of 22 nucleotides, and 200 nucleotides have been used to generate aptamers, the typical range is $40-70$ nucleotides. ${ }^{18,161,275} \mathrm{~A}$ shorter random region allows more sequence space to be sampled, but could lack structural complexity. In contrast, a longer random region samples less sequence space, but may provide more structural complexity which could be important for target-binding. ${ }^{276}$ Longer template lengths cause a more expensive selection, and would increase the cost of any downstream applications. To balance conformations and cost, a 60 nucleotide random region was selected for the Lhomocysteine selection, and was extended to 61 through a PCR insertion. ${ }^{272}$ Since the starting libraries for the Randomized and the Redirected SELEX experiments were based on this Lhomocysteine selection, the template length was kept consistent. Though no statistically significant relationship between template length and aptamer affinity has been found, a shorter template would sample more sequence space, and reduce cost. ${ }^{18}$ The Silverman group has also suggested testing multiple random region lengths during aptamer development. ${ }^{276}$ For these reasons, the template length for the Original SELEX was 80 nucleotides, with a shorter 40-base random region. 


\subsubsection{SELEX Buffer and Primer Selection}

Aptamer binding can often depend on the buffer conditions, with salt concentration, and $\mathrm{pH}$ affecting aptamer folding. ${ }^{18}$ As two of these SELEX experiments for L-serine were derived from a previous selection for L-homocysteine, the SELEX buffer was kept consistent with 50 $\mathrm{mM}$ Tris- $\mathrm{HCl}, 0.5 \mathrm{M} \mathrm{NaCl}$, and $5 \mathrm{mM} \mathrm{MgCl}_{2} .{ }^{272}$ The downstream application for L-serinebinding aptamers is for use in fertilizer systems. With this in mind, soil solution mixed 1:1 with SELEX buffer was used in the final rounds of selection to ensure selected aptamers could bind L-serine in soil-like conditions. The primers used in the L-homocysteine selection were also used for the Randomized and Redirected SELEX experiments, but primers optimized by another group were used for the Original pool SELEX in order to avoid contamination. ${ }^{272,277}$

\subsubsection{Monitoring SELEX Progression}

In order to monitor the progress of each selection, each DNA library was tagged with fluorescein during PCR. Using this fluorescence, the proportion of DNA binding the target could be quantified relative to the fluorescence of the whole SELEX pool. As L-serine is a small molecule target, non-specific binding to the sepharose resin during these selections could be significant. To minimize this non-specific binding, a "negative selection", in which each SELEX pool was incubated with sepharose resin, was conducted prior to every "positive selection" where the pool was introduced to L-serine-bound sepharose. Throughout the selections, stringency was increased by decreasing the amount of target, and decreasing the incubation time of the pool and target. The ease of L-serine's conjugation to the NHS-sepharose resulted in a high concentration of target exposed to the DNA library, and a low selective pressure. The sepharose resin was diluted $100 \times$ to reduce the concentration of L-serine, and the concentration of the DNA library was increased to add selective pressure. This step is indicated for each 
SELEX experiment in Figure 2.8 to Figure 2.10. In addition, counter selections against similar amino acids L-glycine and L-threonine were performed in the penultimate selection rounds, while the final selection rounds were done in a 1:1 mixture of soil solution and SELEX buffer. The specific conditions of each SELEX round are listed in Table 2.2. The original SELEX was conducted for 11 rounds, showing an overall increase in DNA binding, seen in Figure 2.8.

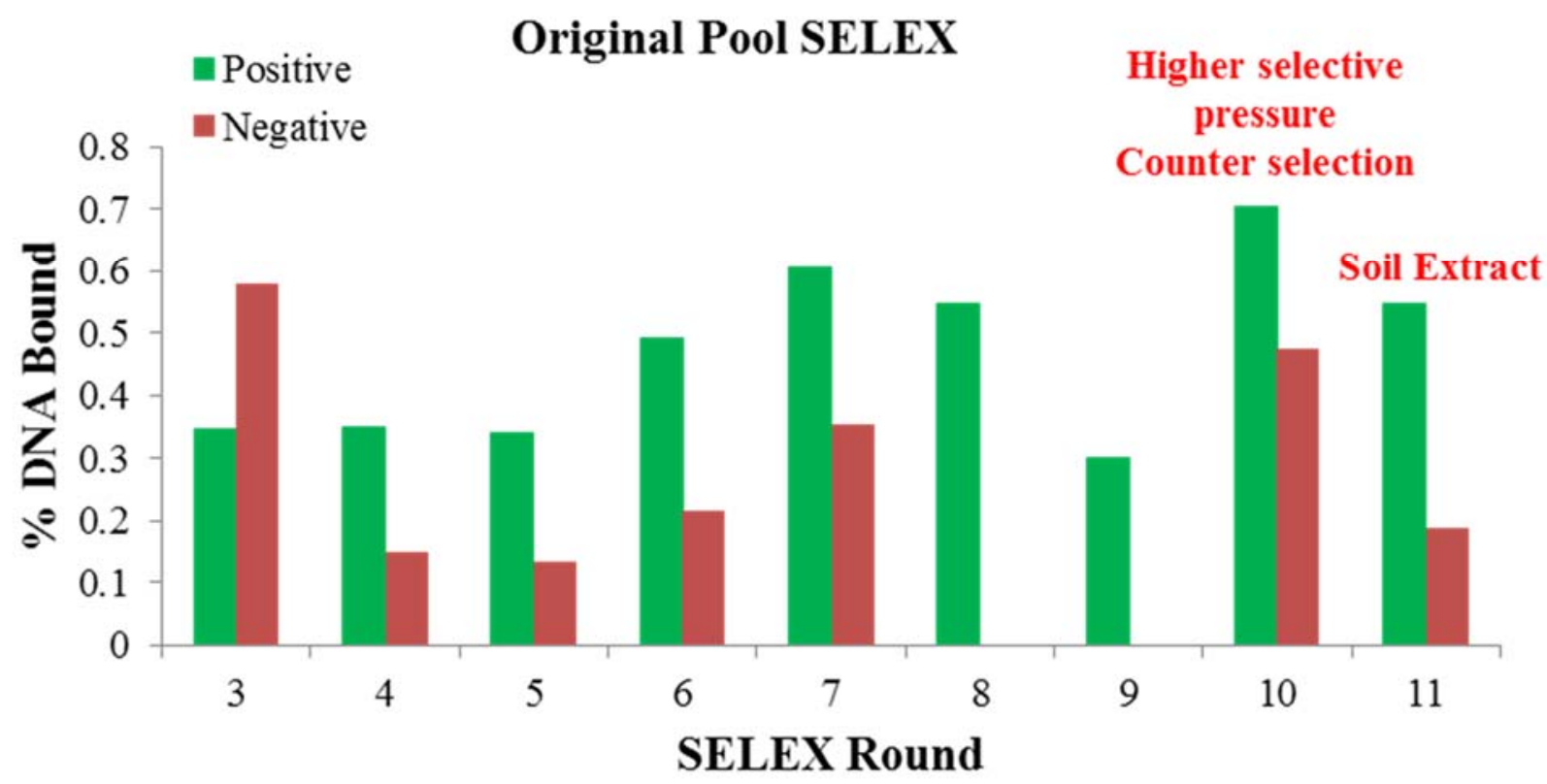

Figure 2.8: From the Original SELEX, the percent recovery of the ssDNA bound to the Lserine-immobilized sepharose (green) and the control Tris-blocked sepharose (red) after each selection round. In Round 10, the selective pressure was increased by reducing the amount of target, and counter selections were done against $L$-glycine and $L$-threonine prior to the positive selection. The final round was completed in soil extract.

Over rounds 3-5, the amount of DNA eluted remained consistent, suggesting that dropping the incubation time from 1 hour to $30 \mathrm{~min}$ did not affect DNA binding. The incubation time was then lowered to 20 minutes in round 7 , and still an increase in binding was observed. This suggests that lowering the incubation time did not hinder target-binding. The proportion of DNA bound to the negative column was higher than the proportion of DNA bound to the positive column in the first measured round (round 3), showing that non-specific binding of the DNA 
pool to the sepharose resin was prevalent. As L-serine is such a small molecule, the sepharose resin provides many more binding sites available for interaction with the DNA pool. The proportion of DNA bound to the negative column decreased significantly in subsequent rounds, showing the use of the negative column in every round was an efficient way to decrease nonspecific binding. In an effort to combat non-specific binding further, two negative columns were used in rounds 9 and 10, but no significant change was observed.

The Randomized SELEX was conducted for 8 rounds, using a pool generated from base substitutions in the Hcy 10 aptamer, and the progression of this SELEX experiment is shown in Figure 2.9.

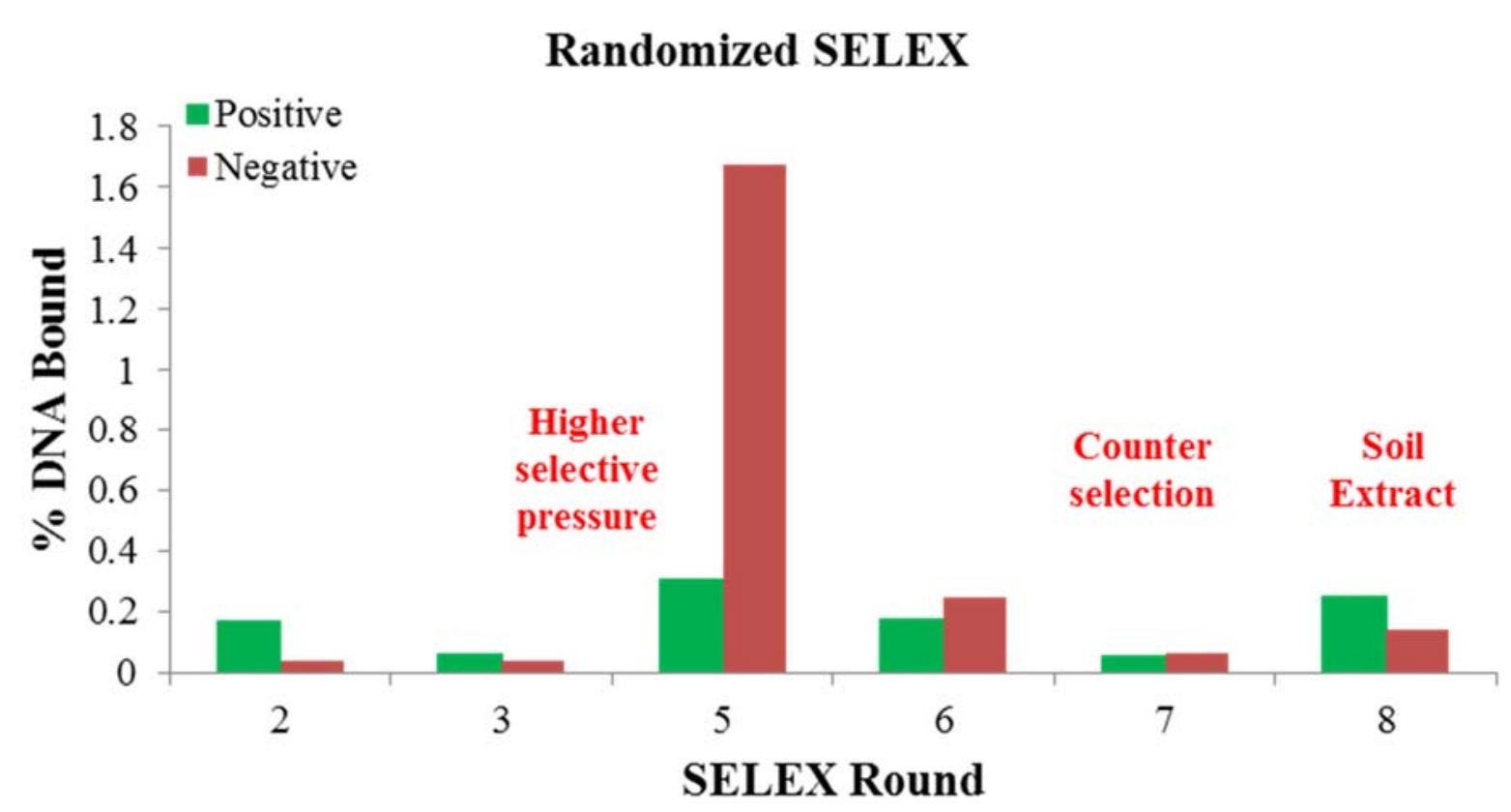

Figure 2.9: From the Randomized SELEX, the percent recovery of the ssDNA bound to the L-serine-immobilized sepharose (green) and the control Tris-blocked sepharose (red) after each selection round. In Round 4 (data not shown), the selective pressure was increased by reducing the amount of target. Counter selections were done against L-glycine and Lthreonine prior to the positive selection in Round 7 . The final round was completed in soil extract. 
The Hcy 10 aptamer had previously undergone 8 rounds of selection using Lhomocysteine-conjugated sepharose beads, and had therefore undergone 8 negative selections. It was likely for this reason that the proportion of DNA binding the negative column was already lower than the positive column in round 2, unlike in the Original Pool SELEX, Figure 2.8. Since the Hcy10 aptamer was randomized by $15 \%$, new sequences not present in the previous selection could exist in this pool with affinity for the sepharose, so negative incubation steps were continued. The proportion of DNA binding the sepharose stayed constant except in round 5, where it increased. In round 4 (not measured), the stringency of the selection was increased by reducing the target concentration 100x, and the effect of this was measured in round 5 , as sequences with weaker affinity would be removed in this step. The DNA binding the positive column stayed relatively consistent, lowering slightly after counter selections with similar small molecules, L-glycine and L-threonine. The DNA pool maintained binding to the positive column when the selection was completed in soil solution in the final round (round 8).

The Redirected SELEX was performed for 5 rounds. The starting library for this selection was made by combining the last two selection rounds (rounds 7 and 8 ) from a previous small molecule selection for L-homocysteine. ${ }^{272}$ The progression of this SELEX experiment is shown in Figure 2.10. 


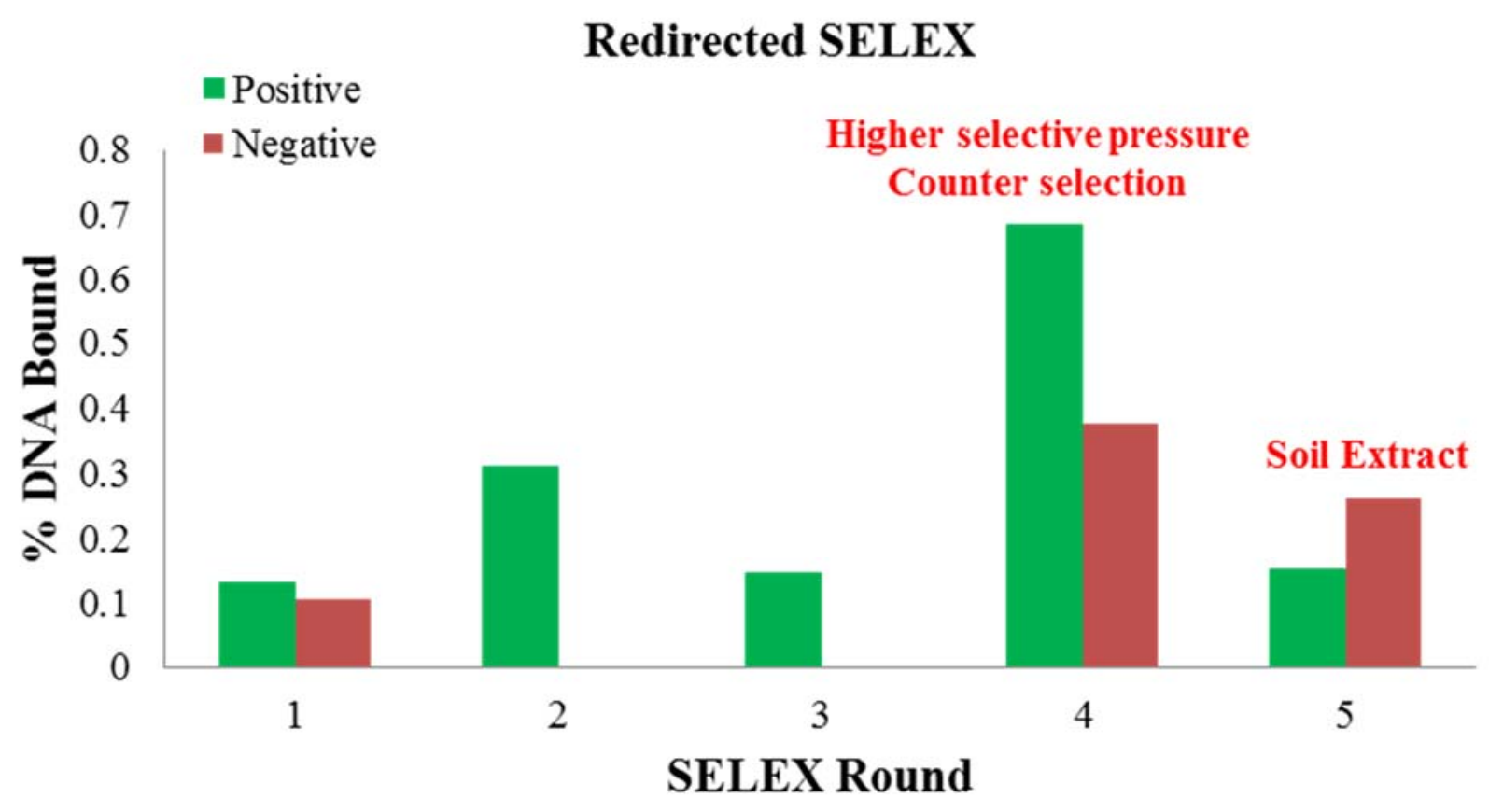

Figure 2.10: From the Redirected SELEX, the percent recovery of the ssDNA bound to the L-serine-immobilized sepharose (green) and the control Tris-blocked sepharose (red) after each selection round. In Round 4, the selective pressure was increased by reducing the amount of target, and counter selections were done against L-glycine and L-threonine prior to the positive selection. The final round was completed in soil extract.

These DNA libraries had undergone negative selections against sepharose, as well as counter selections against L-cysteine and L-methionine, and this selection had generated an aptamer candidate, Hcy10, which showed some binding to L-serine. The last two rounds of the selection were combined and used as the starting pool for the L-serine selections to see if more L-serine-binding candidates existed in these pools. The highest proportion of DNA bound to the positive column was in the fourth round in this redirected SELEX (Figure 2.10) and was greater than the highest proportion bound in the randomized SELEX (Figure 2.9) which could suggest that more L-serine-binding aptamer candidates exist in this redirected pool. As seen in the original SELEX, the proportion of DNA binding L-serine seemed to increase despite counter selections against L-glycine and L-threonine, but decreased when selected in soil extract. Using a complex matrix such as the soil extract in the final selection round would have compromised the 
specificity of the selection, however the use of HTS facilitated the computational elimination of non-specific sequences.

In all three SELEX experiments, DNA sequences with affinity for L-serine were able to withstand a counter selection step against L-glycine and L-threonine, as well as selection in soil solution. The resulting enriched DNA pools should produce aptamer candidates more selective for L-serine, and able to bind L-serine in soil-like conditions. The amount of DNA eluted with affinity for L-serine in each selection experiment was very low, under $1 \%$. High-throughput sequencing was employed to maximize the chances of identifying L-serine-binding aptamers in such a small proportion of the DNA library.

\subsubsection{High-throughput Sequencing (HTS)}

\subsubsection{Setting up HTS Analysis}

In all three selections, the proportion of the DNA pools binding L-serine was very small, under $1 \%$. In order to successfully identify an L-serine-binding aptamer in that $1 \%$, high throughput sequencing was used to screen as many sequences as possible. Traditionally, aptamer candidates were obtained by cloning the last SELEX round, as was done for the L-homocysteine selection. ${ }^{272}$ In the L-homocysteine selection, only 4 different sequences were obtained in 12 bacterial clones, and the best aptamer candidates were identified based on the frequency each sequence was identified. If this low throughput method was used in these L-serine selections, it is unlikely a binding sequence could be found with such a small proportion of DNA binding the target in each round. Using high throughput sequencing allows millions of sequences to be analyzed and compared to previous selection rounds, allowing not only the high frequency sequences to be identified, but also to identify the sequences that became enriched over the course of the selection. HTS analysis of the non-specific binders generated from the negative and 
counter selection rounds allows for their computational deletion from the positive selection rounds. Eliminating the non-specific sequences facilitates the identification of target-binding sequences, rather than sequences that bound the solid support, but were not eliminated during the selection.

From each SELEX experiment, four pools were sent for high throughput sequencing analysis, described in Table 2.3. The starting pools were analyzed for sequence diversity and base distribution in order to mark a starting point to monitor enrichment in subsequent rounds. The last selection round completed in SELEX buffer was analyzed to find aptamer candidates most enriched in buffer, which also made it through counter selections against L-glycine and Lthreonine. The sequences identified from the final rounds of the selections made it through these counter selections, as well as selection in soil solution, hopefully producing sequences selective for L-serine that can bind in soil-like conditions. For each selection, a "negative" round was also sequenced, allowing the sequences binding unwanted targets such as Tris, the sepharose matrix, and counter targets L-glycine and L-threonine to be removed from the analysis of L-serinebinding aptamer candidates.

For each SELEX experiment, at least 48 million sequences were read in the Illumina sequencing process. Each sequencing experiment contained 4 different DNA pools from different SELEX rounds that could be identified based on their Illumina adaptors. Figure 2.11 shows the proportion of each SELEX pool represented in the sequencing experiments. 

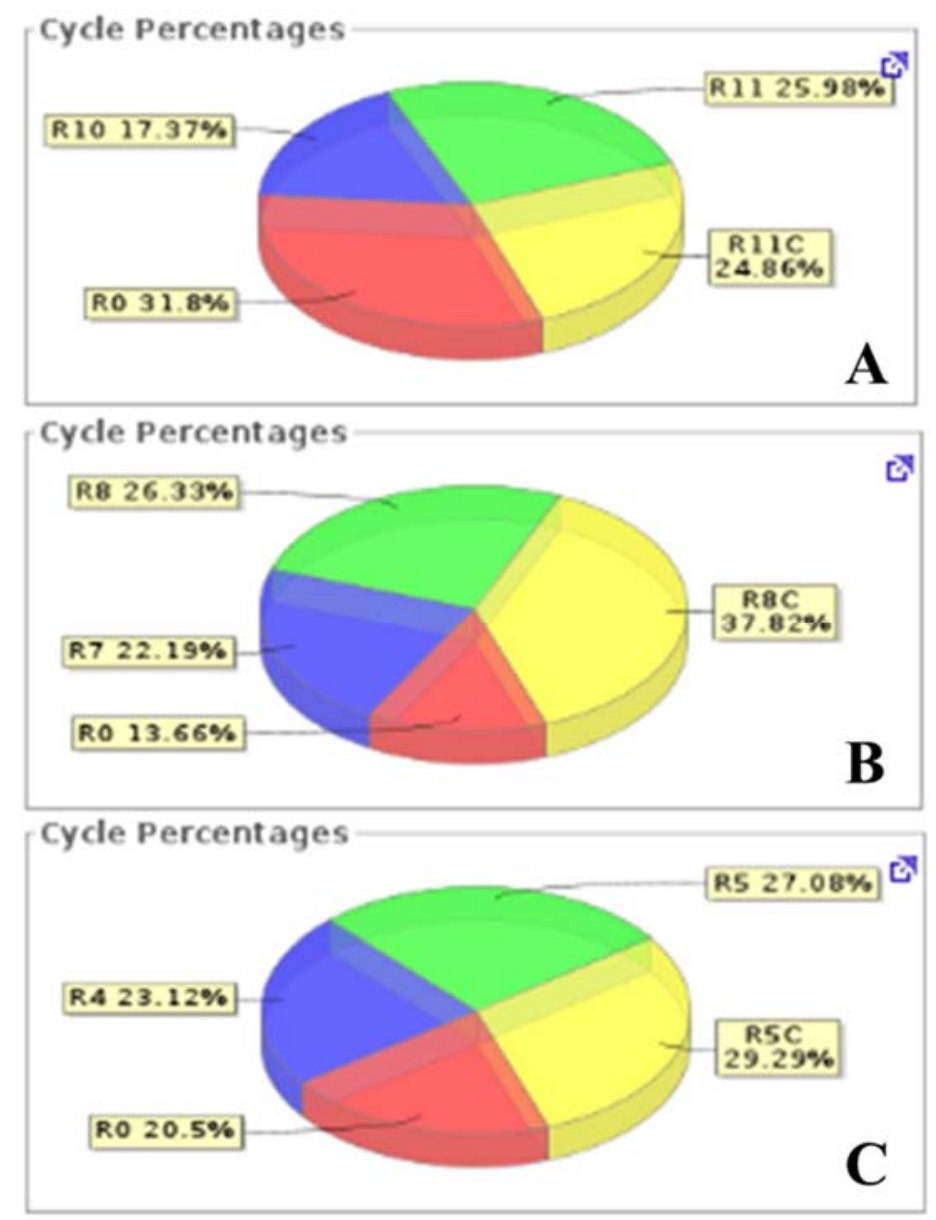

Figure 2.11: The pool size represented in the sequencing reaction from each SELEX round from (A) the Original SELEX; (B) the Randomized SELEX; and (C) the Redirected SELEX.

It was important to ensure that each SELEX pool was sufficiently represented. It was also possible that the size of the random region could change from the original pool, as it did in the homocysteine selection, from 60 bases to 61 due to an advantageous insertion during PCR. ${ }^{272}$ AptaCluster allows the random region size to be examined, but in this case, all selections maintained the random region size from the starting pool, with no insertions or deletions.

\subsubsection{Monitoring Enrichment using AptaCluster}

AptaCluster software can provide a global overview of the selection progress over the rounds sequenced. Figure 2.12 to Figure 2.14 show the progression of the sequence diversity in 
each selection experiment. "Singletons" describe the percentage of sequences with a count of 1 in a selection round, while the "Unique Fraction" describes the percentage of unique sequences as it related to the pool size. The "Enriched" fraction describes the percentage of sequences with a count higher than 1 in that selection round. In a successful SELEX experiment, the unique fraction of the pool would decrease over the course of the selection as stringency increased, and the enriched fraction would increase as more target-binding sequences were generated.

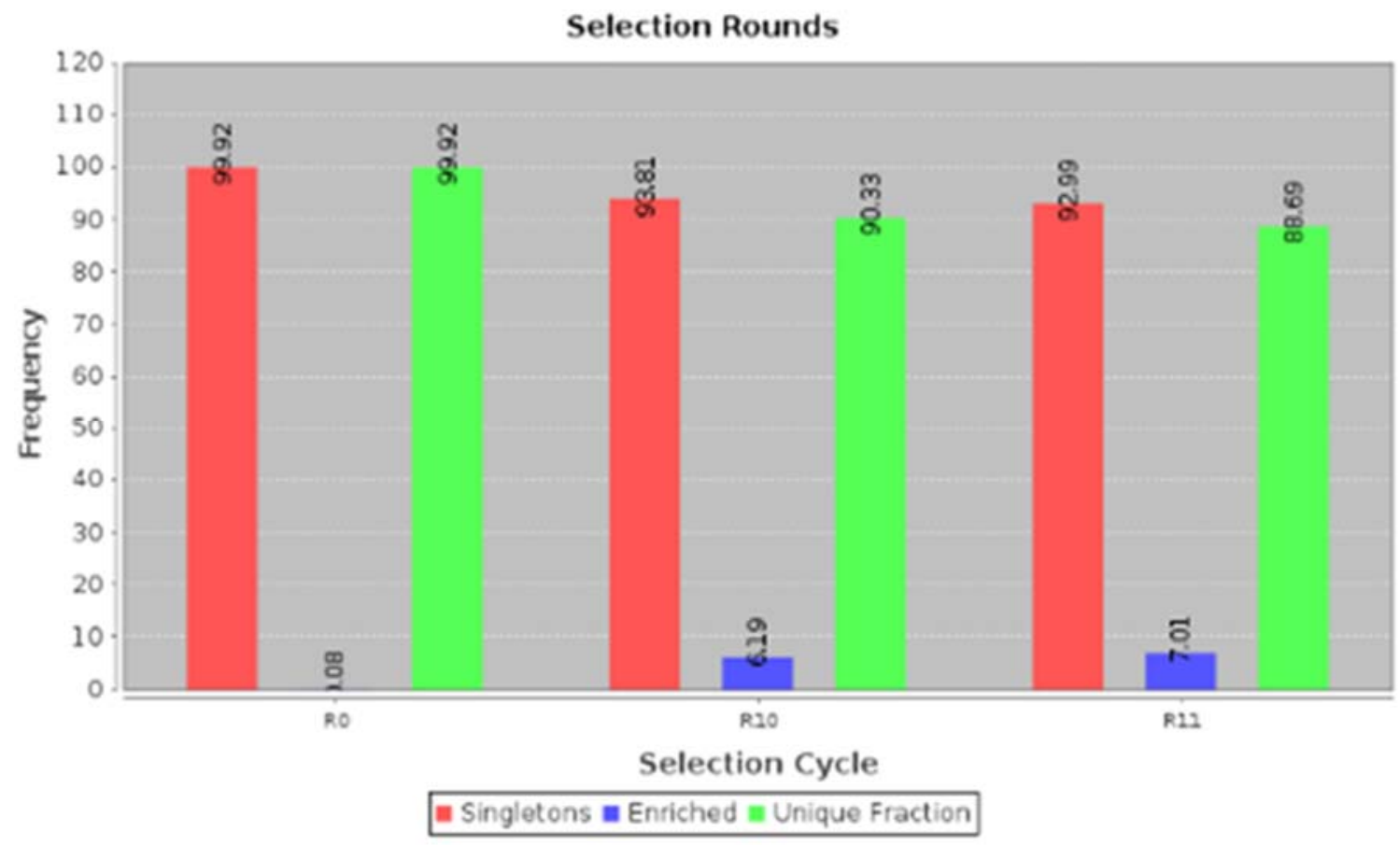

Figure 2.12: Sequence convergence during the Original SELEX after Illumina high throughput sequencing and analysis using AptaCluster and AptaGUI software. 


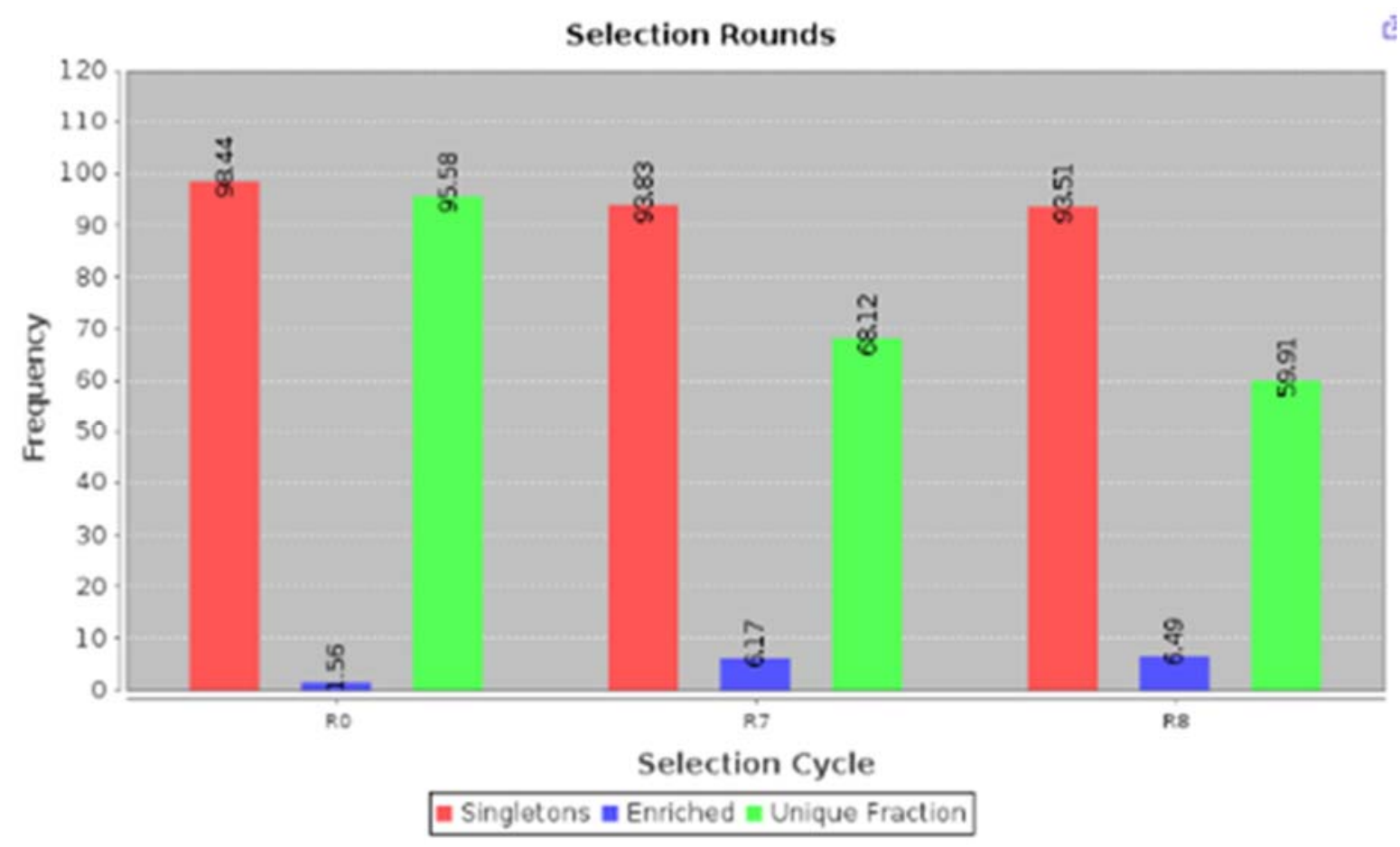

Figure 2.13: Sequence convergence during the Randomized SELEX after Illumina high throughput sequencing and analysis using AptaCluster and AptaGUI software.

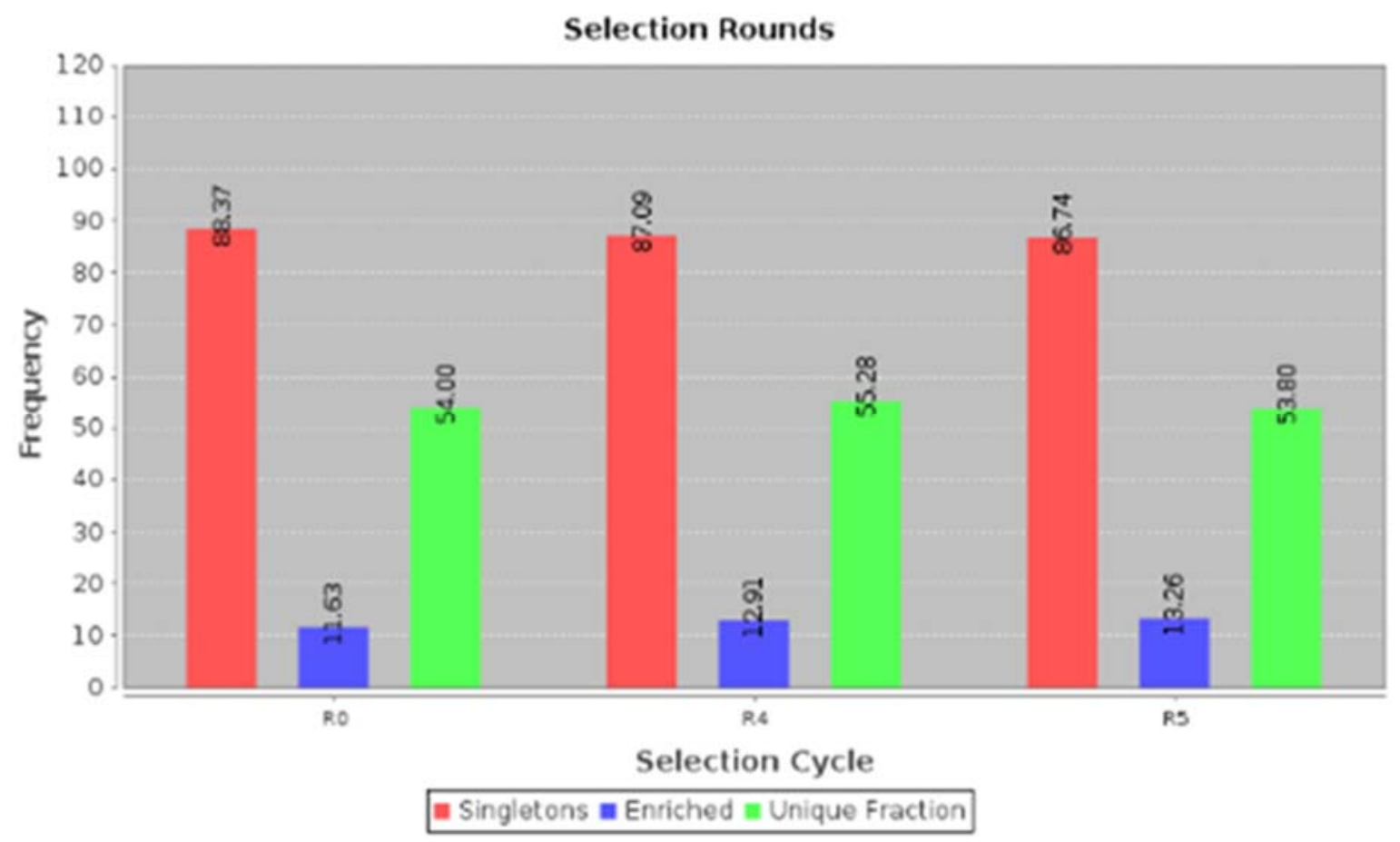

Figure 2.14: Sequence convergence during the Redirected SELEX after Illumina high throughput sequencing and analysis using AptaCluster and AptaGUI software. 
Table 2.5: The base distribution of each SELEX round throughout each SELEX experiment

\begin{tabular}{|c|c|c|c|c|c|c|c|c|c|c|c|}
\hline \multicolumn{3}{|c}{ Original SELEX } & \multicolumn{4}{c}{ Randomized SELEX } & \multicolumn{4}{c|}{ Redirected SELEX } \\
\hline Base & R0 & R10 & R11 & Base & R0 & R7 & R8 & Base & R0 & R4 & R5 \\
\hline A & 27.4 & 29.1 & 29.4 & A & 27.9 & 30.2 & 30.5 & A & 32.6 & 32.7 & 32.6 \\
\hline T & 30.4 & 25.6 & 25.8 & T & 21.5 & 22.2 & 22.1 & T & 20.5 & 20.5 & 20.8 \\
\hline C & 23.4 & 33.2 & 32.7 & C & 16.4 & 16.1 & 16.9 & C & 21.6 & 21.4 & 21.3 \\
\hline G & 18.7 & 12.2 & 12 & G & 34.2 & 31.5 & 30.6 & G & 25.3 & 25.4 & 25.3 \\
\hline
\end{tabular}

In the Original SELEX experiment, the unique fraction of the starting round (R0) was $99.92 \%$, giving a good indication that the starting library was diverse, with very few repeated sequences. After ten rounds of selection, this unique fraction decreased to $90.33 \%$, and after the final round (R11) to $88.69 \%$. The enrichment increased from nearly zero $(0.08 \%)$ in the starting pool, to $7.01 \%$ in round 11 . Both the decreasing unique fraction, and increasing enriched portion of the SELEX pools suggested that L-serine-binding sequences were getting enriched, while non-binding sequences were being removed from the pool. Table 2.5 shows the overall base distribution of the sequences in each round of the selection. In the starting pool of the original SELEX, nucleotide T was slightly over-represented at $30.4 \%$, while nucleotide $\mathrm{G}$ was slightly under-represented at $18.7 \%$. The frequency of $\mathrm{G}$ and $\mathrm{T}$ continued to decrease over the selection, while the frequency of $\mathrm{A}$ and $\mathrm{C}$ increased. These changes in base distribution in favour of $\mathrm{A}$ and C could suggest that these nucleotides more readily participated in structures important for target binding.

In the Randomized SELEX experiment, the unique fraction of the starting round (R0) was $95.58 \%$ which was less diverse than the original pool. This lower diversity of the starting pool could be attributed to the way in which the pool was synthesized, as all the sequences were derived from the existing Hcy10 aptamer, by randomizing the aptamer's random region by $15 \%$. 
The synthesis of this pool was biased towards the Hcy10 sequence, which generated a less diverse pool. Considering the pool was created from one unique sequence, this level of sequence diversity was high. After seven rounds of selection, the unique fraction decreased to $68.12 \%$, and to $59.91 \%$ by the final round (R8). This was the largest drop in the unique fraction seen for any of the selections, suggesting the largest change in sequence diversity occurred during this randomized SELEX experiment. The base distribution for this selection is shown in Table 2.5, but did not show a large change over the 8 selection rounds. There was a slight decrease in the proportion of $\mathrm{G}$, and a slight increase in the proportion of $\mathrm{A}$ represented in these sequences by the final round.

In the Redirected SELEX experiment, the unique fraction of the starting pool was $54.00 \%$ which was the lowest diversity of the three starting pools. This redirected pool was obtained by combining rounds 7 and 8 of an L-homocysteine selection experiment. During this L-homocysteine selection, the enrichment of the pool increased over 8 rounds, lowering the sequence diversity of the pool used as a starting pool in this L-serine selection. Over four additional rounds of selection for L-serine, the unique fraction of the pool actually increased to $55.28 \%$ before ending at $53.80 \%$ in round 5 , only slightly less diverse than the starting pool. This plateau in the sequence diversity could suggest that the starting pool was already so enriched for L-homocysteine binding sequences that it was unable to be redirected to L-serine binding sequences. It is also possible that five selection rounds were not enough to have an effect on the sequence diversity. The base distribution shown in Table 2.5 remained largely unchanged throughout this redirected SELEX, also suggesting no change in sequence diversity. Despite this plateau in the unique fraction, the enriched fraction did show a slight increase from $11.63 \%$ to 
$13.26 \%$ over the five selection rounds, so L-serine-binding sequences may still have been enriched.

\subsubsection{Identifying aptamer candidates using AptaCluster}

AptaCluster is able to sort DNA sequences by their count, which is the frequency the sequence appears in the pool, or by their enrichment, which is the fold change in the frequency of the sequence with respect to pool size. Sequences with the highest count may represent the best aptamer candidate, but may also represent sequences which amplified more efficiently during PCR. To avoid this, sequences with the highest enrichment were selected, as this value minimized the effects of PCR bias. The aptamer candidates with the highest enrichment from each SELEX experiment are shown in Table 2.6.

Table 2.6: Count and enrichment scores of the best aptamer candidates from each selection, generated using AptaCluster after Illumina high-throughput sequencing.

\section{Original SELEX}

\begin{tabular}{|c|c|c|c|c|c|c|}
\hline \multirow[t]{2}{*}{ Aptamer } & \multicolumn{2}{|c|}{ R11 } & \multicolumn{2}{|c|}{ R11 Control } & \multicolumn{2}{|c|}{ R10 } \\
\hline & Count & Enrichment & Count & Enrichment & Count & Enrichment \\
\hline Ser.1 & $3.2 \times 10^{2}$ & $2.1 \times 10^{2}$ & $2.4 \times 10^{2}$ & $1.7 \times 10^{2}$ & --- & ---- \\
\hline Ser.2 & $8.3 \times 10^{3}$ & $1.2 \times 10^{0}$ & $9.1 \times 10^{3}$ & $1.3 \times 10^{0}$ & $4.7 \times 10^{3}$ & $9.6 \times 10^{3}$ \\
\hline \multicolumn{7}{|c|}{ Randomized SELEX } \\
\hline \multirow[t]{2}{*}{ Aptamer } & \multicolumn{2}{|c|}{ R8 } & \multicolumn{2}{|c|}{ R8 Control } & \multicolumn{2}{|c|}{ R7 } \\
\hline & Count & Enrichment & Count & Enrichment & Count & Enrichment \\
\hline Ran.1 & $6.3 \times 10^{5}$ & $2.6 \times 10^{5}$ & $8.3 \times 10^{1}$ & $2.4 \times 10^{1}$ & $2.0 \times 10^{0}$ & $1.8 \times 10^{-1}$ \\
\hline Ran.2 & $6.2 \times 10^{3}$ & $1.0 \times 10^{0}$ & $4.3 \times 10^{3}$ & $4.9 \times 10^{-1}$ & $5.2 \times 10^{3}$ & $3.2 \times 10^{3}$ \\
\hline \multicolumn{7}{|c|}{ Redirected SELEX } \\
\hline \multirow[t]{2}{*}{ Aptamer } & \multicolumn{2}{|c|}{ R5 } & \multicolumn{2}{|c|}{ R5 Control } & \multicolumn{2}{|c|}{ R4 } \\
\hline & Count & Enrichment & Count & Enrichment & Count & Enrichment \\
\hline Red.1 & $7.9 \times 10^{4}$ & $6.7 \times 10^{4}$ & $2.0 \times 10^{0}$ & $1.6 \times 10^{0}$ & $1.0 \times 10^{0}$ & ---- \\
\hline Red.2 & $1.0 \times 10^{0}$ & 0 & $1.0 \times 10^{0}$ & 0 & $2.0 \times 10^{4}$ & $1.8 \times 10^{4}$ \\
\hline
\end{tabular}

*X.1 sequences were selected as most enriched in soil solution, and X.2 sequences were most enriched in SELEX buffer where $\mathrm{X}$ is the Ser, Ran or Red selection identifiers 
The enrichment of sequence Ser. 1 in the final soil solution round was $2.1 \times 10^{2}$, compared to $1.7 \times 10^{2}$ in the control round which suggested that this sequence showed more binding to the L-serine-conjugated sepharose than to the sepharose alone, L-glycine, or Lthreonine. The count of this Ser.1 sequence was also higher in the soil solution round compared to the control round. Since both the enrichment and count of this sequence were higher in the final selection round than in the controls, Ser.1 was selected as a promising aptamer candidate. Interestingly, this Ser.1 sequence was not represented in the final selection round performed in buffer. A sequence with very high similarity could have been present in the buffer round, and undergone a point mutation to generate Ser.1 in the final round, however software limitations prevented the identification of these sequences. Since this Ser.1 sequence was not present in the buffer round, sequence Ser.2 was selected as it was the most enriched sequence in the final buffer round. Table 2.6 shows the enrichment and count values of the sequences selected for further characterization. Ser.1 (from original SELEX), Ran.1 (from Randomized SELEX), and Red.1 (from Redirected SELEX) were sequences selected due to their high enrichment values in the final soil solution round, compared to a control. This control contained sequences binding Tris-conjugated sepharose, L-glycine, L-threonine, or components of the soil solution. In the event that the soil solution in the final round caused the loss of excellent binding candidates, sequences with the highest enrichment in the final buffer round were also selected for further characterization. Ser.2, Ran.2, and Red.2 represent the sequences most enriched in the final buffer selection round of each SELEX experiment. The sequences of these six aptamer candidates selected for further characterization are shown in Table 2.7. 
Table 2.7: Aptamer candidate sequences generated from each L-serine selection experiment, after Illumina high-throughput sequencing and AptaCluster analysis.

\begin{tabular}{|c|c|c|}
\hline $\begin{array}{l}\text { Aptamer } \\
\text { Candidate }\end{array}$ & Selection Experiment & Sequence \\
\hline Ser.1 & Original SELEX & $\begin{array}{l}\text { AGCAGCACAGAGGTCAGATGCCTATGCGTG } \\
\text { CTACCGTGAAACCGATCGGAAGAGCACACG } \\
\text { CCTATGCGTGCTACCGTGAA }\end{array}$ \\
\hline Ser.2 & Original SELEX & $\begin{array}{l}\text { AGCAGCACAGAGGTCAGATGCGATCTGGAT } \\
\text { ATTATTTTTGATACCCCTTTGGGGAGACATC } \\
\text { CTATGCGTGCTACCGTGAA }\end{array}$ \\
\hline Ran.1 & Randomized HCY10 SELEX & $\begin{array}{l}\text { ATACCAGCTTATTCAATTAGATAGTAAGTGC } \\
\text { AATCTTGATCGGAAGAGCACACGTCTGAACT } \\
\text { CCAGTCACTTAGGCATCAGATAGTAAGTGCA } \\
\text { ATCT }\end{array}$ \\
\hline $\operatorname{Ran} .2$ & Randomized HCY10 SELEX & $\begin{array}{l}\text { ATACCAGCTTATTCAATTGTATACGGAGTGG } \\
\text { ATATCGATCTGTAACGTGAGTGAGATAATGT } \\
\text { GATGCATAGTCGTGGAGAGATAGTAAGTGC } \\
\text { AATCT }\end{array}$ \\
\hline Red.1 & Redirected SELEX & $\begin{array}{l}\text { ATACCAGCTTATTCAATTAGATAGTAAGTGC } \\
\text { AATCTAGATCGGAAGAGCACACGTCTGAAC } \\
\text { TCCAGTCACTTAGGCATCAGATAGTAAGTGC } \\
\text { AATCT }\end{array}$ \\
\hline Red.2 & Redirected SELEX & $\begin{array}{l}\text { ATACCAGCTTATTCAATTGGCCGTGTAGATA } \\
\text { GTAAGTGCAATCTGATCGGAAGAGCACACG } \\
\text { TCTGAACTCCAGTCACCGAGATAGTAAGTGC } \\
\text { AATCT }\end{array}$ \\
\hline
\end{tabular}

\subsubsection{Identification of Hcy10 within the Randomized and Redirected SELEX experiments}

The Randomized SELEX and the Redirected SELEX performed for L-serine were derived from a previous small molecule SELEX for L-homocysteine. ${ }^{272}$ From the initial Lhomocysteine selection, the aptamer Hcy10 was generated, showing some affinity for immobilized L-serine (see section 2.5.2). The goal for the Randomized and Redirected selections was to improve upon this Hcy10 affinity for L-serine. The Randomized SELEX used a pool synthesized by randomizing the Hcy10 aptamer to find beneficial mutations, whereas the 
Redirected SELEX used the last two selection rounds from which Hcy10 was derived in order to find a better binder for L-serine. In both selections, the Hcyl0 aptamer was found in the final rounds, having persisted through each selection. Table 2.8 shows the count and enrichment values of the Hcy10 aptamer in the Randomized and Redirected selections.

Table 2.8: Count and Enrichment scores for the Hcy10 aptamer found in the Randomized and Redirected selection pools, generated using AptaCluster after Illumina highthroughput sequencing.

\section{Randomized SELEX}

\begin{tabular}{ccccccc}
\hline \multirow{2}{*}{ Aptamer } & \multicolumn{2}{l}{ R8 } & \multicolumn{2}{c}{ R8 Control } & \multicolumn{2}{c}{ R7 } \\
\cline { 2 - 7 } & Count & Enrichment & Count & Enrichment & Count & Enrichment \\
\hline Hcy10 & 15 & $3.2 \times 10^{-1}$ & 11 & $1.7 \times 10^{-1}$ & 39 & $3.6 \times 10^{-3}$ \\
\hline \multirow{2}{*}{ Redirected SELEX } & & & & & \\
\hline Aptamer & \multicolumn{2}{c}{ R5 } & \multicolumn{2}{c}{ R5 Control } & \multicolumn{2}{c}{ R4 } \\
\cline { 2 - 8 } & Count & Enrichment & Count & Enrichment & Count & Enrichment \\
\cline { 2 - 8 } Hcy10 & 86 & $7.9 \times 10^{-1}$ & 80 & $6.8 \times 10^{-1}$ & 93 & $9.2 \times 10^{0}$ \\
\hline
\end{tabular}

In both the Randomized and Redirected selections, Hcy10 was more enriched in the final round compared to the control round, showing that the Hcy10 aptamer did bind the L-serinesepharose more than the control, and also bound L-serine when exposed to soil solution. Though the Hcy10 aptamer was present, and more enriched in the final rounds of the Randomized and Redirected selections compared to the control, the aptamer candidates generated through these three L-serine selections shown in Table 2.6 showed much higher enrichment values, suggesting they may have been better aptamer candidates than the Hcy10 aptamer.

As both the Randomized and Redirected SELEX experiments were derived from the Lhomocysteine selection which produced Hcy10, the resulting sequences were examined for homology. The sequence alignment of these aptamer candidates compared to Hcy10 is shown in Table 2.9. 
Table 2.9: Sequence alignment of aptamers derived from L-homocysteine selections compared to Hcy10 aptamer

\begin{tabular}{|c|r|c|c|}
\hline Aptamer & Aptamer sequence ATACCAGCTTATTCAATT-N61-AGATAGTAAGTGCAATCT & Similarity \\
\hline Hey10 & GTGGAAAGCCGAATGTGATTAGGGACCAGTGGAGAAGTAGTACGGACTGACCTCGCGTGTA & to Hcy10 \\
\hline Ran.1 & AGATAGTAAGTGCAATCTTGATCGGAAGAGCACACGTCTGAACTCCAGTCACTTAGGCATC & $19.7 \%$ \\
\hline Ran.2 & GTATACGGAGTGGATATCGATCTGTAACGTGAGTGAGATAATGTGATGCATAGTCGTGGAG & $22.9 \%$ \\
\hline Red.1 & AGATAGTAAGTGCAATCTAGATCGGAAGAGCACACGTCTGAACTCCAGTCACTTAGGCATC & $18.0 \%$ \\
\hline Red.2 & GGCCGTGTAGATAGTAAGTGCAATCTGATCGGAAGAGCACACGTCTGAACTCCAGTCACCG & $18.0 \%$ \\
\hline
\end{tabular}


Aptamer candidates Ran.1 and Ran.2 were most similar to Hcy 10 with $19.7 \%$ and $22.9 \%$ similarity, respectively. As these aptamer candidates were derived from the library which had mutated Hcy10 by only15\%, and Hcy10 did show some binding to immobilized L-serine (as seen in section 2.5.2), a higher sequence similarity was expected. In this library, the Hcy10 aptamer was given a large selective advantage, and yet the successful aptamer candidates bore

little resemblance to Hcy10, which suggested the Hcy10 sequence was selected against. The candidates Red.1 and Red.2 also bore little resemblance to Hcy 10 with only $18 \%$ sequence similarity. This low sequence similarity to Hcy 10 suggested that a large deviation from the original sequence, rather than point mutations, improved L-serine-binding. These selections could have favoured new sequence motifs not seen in Hcy10 which improved binding to Lserine.

\subsubsection{Analysis of Sequence Homology}

\subsubsection{Identification of long conserved sequence motifs}

As all three SELEX experiments were performed for L-serine as the target, an examination of any common sequence motifs present in the resulting aptamer candidates could help elucidate possible binding motifs. Common sequence motifs were identified using a pairwise sequence alignment tool (http://www.ebi.ac.uk/Tools/psa/emboss_needle/nucleotide.html) and all sequences were manually screened for common motifs. Large conserved sequence motifs are shown in Table 2.10 . 
Table 2.10: Conserved sequence motifs (>10 nucleotides) in each L-serine-binding aptamer candidate and Hcy10.

\begin{tabular}{|c|c|c|}
\hline $\begin{array}{l}\text { Aptamer } \\
\text { Candidate }\end{array}$ & Selection Experiment & Sequence \\
\hline Ser.1 & Original SELEX & $\begin{array}{l}\text { AGCAGCACAGAGGTCAGATGCCTATGCGTGCTACCGTGAAACCGATCGG } \\
\text { AAGAGCACACGCCTATGCGTGCTACCGTGAA }\end{array}$ \\
\hline Ser.2 & Original SELEX & $\begin{array}{l}\text { AGCAGCACAGAGGTCAGATGCGATCTGGATATTATTTTTGATACCCCTTT } \\
\text { GGGGAGACATCCTATGCGTGCTACCGTGAA }\end{array}$ \\
\hline Ran.1 & Randomized SELEX & $\begin{array}{l}\text { ATACCAGCTTATTCAATTAGATAGTAAGTGCAATCTTGATCGGAAGAGCA } \\
\text { CACGTCTGAACTCCAGTCACTTAGGCATCAGATAGTAAGTGCAATCT }\end{array}$ \\
\hline Ran.2 & Randomized SELEX & $\begin{array}{l}\text { ATACCAGCTTATTCAATTGTATACGGAGTGGATATCGATCTGTAACGTGA } \\
\text { GTGAGATAATGTGATGCATAGTCGTGGAGAGATAGTAAGTGCAATCT }\end{array}$ \\
\hline Red.1 & Redirected SELEX & $\begin{array}{l}\text { ATACCAGCTTATTCAATTAGATAGTAAGTGCAATCTAGATCGGAAGAGC } \\
\underline{\text { ACACGTCTGAACTCCAGTCACTTAGGCATCAGATAGTAAGTGCAATCT }}\end{array}$ \\
\hline Red.2 & Redirected SELEX & $\begin{array}{l}\text { ATACCAGCTTATTCAATTGGCCGTGTAGATAGTAAGTGCAATCTGATCGG } \\
\text { AAGAGCACACGTCTGAACTCCAGTCACCGAGATAGTAAGTGCAATCT }\end{array}$ \\
\hline Hсy10 & $\begin{array}{l}\text { Homocysteine } \\
\text { SELEX }^{272}\end{array}$ & $\begin{array}{l}\text { ATACCAGCTTATTCAATTGTGGAAAGCCGAATGTGATTAGGGACCAGTG } \\
\text { GAGAAGTAGTACGGACTGACCTCGCGTGTAAGATAGTAAGTGCAATCT }\end{array}$ \\
\hline
\end{tabular}

The forward and reverse primer binding sites are indicated in green and blue, respectively for the Original SELEX, and in mauve and yellow, respectively for the Randomized and Redirected SELEX experiments and Hcy10. Conserved motifs are shown in red and underlined. 
When examining the sequences obtained from the original SELEX experiment, the reverse primer binding site (5'-CCTATGCGTGCTACCGTGAA-3') was found within the central sequence of aptamer candidate Ser.1, immediately following the forward primer binding site, shown in Table 2.10. Interestingly, the reverse primer binding site from the Randomized and Redirected SELEX experiments (5'-AGATAGTAAGTGCAATCT-3') also appeared immediately following the forward primer binding sites in aptamer candidates Ran.1 and Red.1. In sequence Red.2, this sequence also appeared, however slightly more shifted towards the 3' end. It was unexpected to observe this appearance of the reverse primer binding site in three separate SELEX experiments with two different sets of primers. Both primer sets were examined for their likelihood to form primer-dimers using the Multiple Primer Analyzer tool (ThermoFisher Scientific), and no primer-dimers were found. During the selection experiments, no change in library size was observed for any of the SELEX experiments to indicate mispriming. As these reverse primer binding regions appeared in many of the most promising aptamer candidates, it was possible that these sequences participated in target-binding.

Appreciable sequence homology was observed among the aptamer candidates. Sequences Ran.1 and Red.1 were identical except for nucleotide 37. As these sequences were obtained from two different selection experiments, this sequence was a good candidate for further characterization. Sequence Red.2 was also very similar to Ran.1 and Red.1. In all three sequences, there was a conserved motif of 37 nucleotides (5'-

GGAAGAGCACACGTCTGAACTCCAGTCACTTAGGCATC-3'), shown in red in Table 2.10. Interestingly, a 13-nucleotide portion of this motif (5'-GGAAGAGCACACG-3') was found in sequence Ser.1 which was obtained from the Original Pool SELEX. As this original selection had no bias and still produced a conserved motif observed in candidates from each 
SELEX experiment, this motif could be important for L-serine binding. The aptamers that had been previously selected for amino-acid targets (listed in Table 2.1) were manually examined for the presence of this motif. The 7-nucleotide sequence 5'-GGAAGAG-3', which is a portion of this long motif, was identified in the middle section of an L-arginine-binding aptamer selected by Geiger et al. ${ }^{167}$ This suggests that this 5'-GGAAGAG-3' motif could have affinity for amino acid structure.

Since many aptamer candidates shared long sequence motifs, it was interesting to examine the persistence of these motifs throughout the selection experiments. AptaCluster software displays the percentage of each selection round that represents unique sequences (termed "Unique Fraction"). The total number of unique sequences in each SELEX round could be found by multiplying this percentage by each library size. Each conserved sequence motif was then searched for within AptaCluster software to reveal the total number of unique sequences containing each motif in each selection round. With these values, the percentage of unique sequences which contain each conserved motif could be calculated, and are displayed in Table 2.11 
Table 2.11: Persistence of conserved sequence motifs from the starting libraries to the final rounds in each SELEX experiment

\begin{tabular}{|c|c|c|c|c|}
\hline \multirow[t]{2}{*}{ SELEX Experiment } & \multirow[t]{2}{*}{ Conserved Sequence Motif } & \multicolumn{2}{|c|}{$\%$ of Unique Sequences } & \multirow[t]{2}{*}{ Increase $(\mathrm{X})$} \\
\hline & & $\begin{array}{l}\text { Starting } \\
\text { Library }\end{array}$ & $\begin{array}{l}\text { Final SELEX } \\
\text { Round }\end{array}$ & \\
\hline \multirow[t]{3}{*}{ Original SELEX } & $\begin{array}{c}\text { Reverse primer-binding site } \\
\text { (5'-CCTATGCGTGCTACCGTGAA-3') }\end{array}$ & $2.1 \times 10^{-3}$ & $2.3 \times 10^{-1}$ & 110 \\
\hline & $\begin{array}{l}\text { Forward primer-binding site } \\
\text { (5'-AGCAGCACAGAGGTCAGATG-3') }\end{array}$ & $7.1 \times 10^{-5}$ & $1.4 \times 10^{-4}$ & 2 \\
\hline & 5'-GGAAGAGCACACG-3' & $1.7 \times 10^{-4}$ & $3.3 \times 10^{-3}$ & 28 \\
\hline \multirow[t]{3}{*}{ Randomized SELEX } & $\begin{array}{l}\text { Reverse primer-binding site } \\
\text { (5'-AGATAGTAAGTGCAATCT-3') }\end{array}$ & $2.5 \times 10^{-2}$ & $1.7 \times 10^{0}$ & 68 \\
\hline & $\begin{array}{l}\text { Forward primer-binding site } \\
\text { (5'-ATACCAGCTTATTCAATT-3') }\end{array}$ & $7.7 \times 10^{-3}$ & $6.8 \times 10^{-1}$ & 88 \\
\hline & 5'-GGAAGAGCACACG-3' & $8.6 \times 10^{-3}$ & $4.2 \times 10^{-1}$ & 49 \\
\hline \multirow[t]{3}{*}{ Redirected SELEX } & $\begin{array}{l}\text { Reverse primer-binding site } \\
\text { (5'-AGATAGTAAGTGCAATCT-3') }\end{array}$ & $7.1 \times 10^{0}$ & $1.4 \times 10^{1}$ & 2 \\
\hline & $\begin{array}{c}\text { Forward primer-binding site } \\
\text { (5'-ATACCAGCTTATTCAATT-3') }\end{array}$ & $4.3 \times 10^{0}$ & $9.5 \times 10^{0}$ & 2 \\
\hline & 5'-GGAAGAGCACACG-3' & $2.1 \times 10^{0}$ & $3.8 \times 10^{0}$ & 2 \\
\hline
\end{tabular}


In the Original SELEX experiment, the conserved motifs made up a very small percentage of the starting library, demonstrating the large diversity that was present in the original pool starting library, shown in Table 2.11. Throughout the selection, the presence of the reverse primer-binding region increased by 110 times. While this region was likely introduced through PCR, the selection showed some preference for this reverse primer-binding region, since the presence of the forward primer-binding region only increased by 2 times by the final round. The motif 5'-GGAAGAGCACACG-3' which was found in four of the aptamer candidates was also shown to be 28 times more abundant by the final round, which could suggest an affinity for L-serine. The Randomized SELEX experiment also showed the persistence of this motif, as it became 49 times more abundant by the final selection round. In the Randomized SELEX, both primer-binding regions became more abundant throughout the selection.

It was interesting to examine these conserved motifs in the Redirected SELEX experiment, as each motif was very abundant in the starting SELEX library. The reverse primerbinding site, the forward primer-binding site, and the motif 5'-GGAAGAGCACACG-3' were found in $7.1 \%, 4.3 \%$, and $2.1 \%$ of the sequences in the redirected starting library, which was at least three orders of magnitude higher than the Original SELEX. This was indicative of lower sequence diversity for the Redirected SELEX. The starting library for this Redirected SELEX experiment came from the final rounds of an L-homocysteine selection, so it was likely these motifs were enriched during the L-homocysteine selection. Though these motifs were enriched during an L-homocysteine selection, they became two times more abundant by the final L-serine selection round. 


\subsubsection{Identification of short conserved sequence motifs}

The aptamer candidates were compared to identify shorter regions of homology (4-10 nucleotides), to identify possible L-serine-binding motifs. Common sequence motifs were identified using a pairwise sequence alignment tool (http://www.ebi.ac.uk/Tools/psa/emboss_needle/nucleotide.html) and all sequences were manually screened for common motifs. Shorter conserved motifs derived from the randomized region of the pools are found in Table 2.12, while short conserved motifs involving the primerbinding sites are shown in Table 2.13. 
Table 2.12: Conserved sequence motifs (4-10 nucleotides) which exclude the primer-binding regions, appearing in at least 2 Lserine-binding aptamer candidates or Hcy10.

\begin{tabular}{|c|c|c|c|c|c|c|c|}
\hline \multirow{2}{*}{ Domain } & \multicolumn{7}{|c|}{ Nucleotide Position in DNA Sequence from 5' end } \\
\hline & Ser.1 & Ser.2 & Ran.1 & Ran.2 & Red.1 & Red.2 & Hcy10 \\
\hline CGATCTG & --- & 21 & --- & 36 & --- & --- & --- \\
\hline CGATC & 43 & 21 & $\begin{array}{ll}-- \\
\end{array}$ & 36 & --- & --- & --- \\
\hline -GATC-- & 44 & 22 & 38 & 37 & 38 & 45 & --- \\
\hline -TGGATAT & --- & 26 & --- & 29 & --- & --- & --- \\
\hline GTGGA & --- & --- & --- & 28,74 & --- & --- & 19,47 \\
\hline -TGGA--- & --- & 26 & --- & 29,75 & --- & --- & 20,48 \\
\hline GGAGA & --- & 53 & --- & 76 & --- & --- & 48 \\
\hline TGAA & 37,77 & 77 & 57 & --- & 57 & 64 & --- \\
\hline GAAG & 49 & --- & 43 & --- & 43 & 50 & 52 \\
\hline TGAT & --- & 39 & 37 & 62 & --- & 44 & 34 \\
\hline ACGT & --- & --- & 52 & 45 & 52 & 59 & --- \\
\hline CTGA & --- & --- & 56 & --- & 56 & 63 & 65 \\
\hline CATC & --- & 58 & 76 & --- & 76 & --- & --- \\
\hline GCCG & --- & --- & --- & --- & --- & 20 & 26 \\
\hline
\end{tabular}


Table 2.13: Conserved sequence motifs (4-10 nucleotides) which include the primer-binding regions, appearing in at least 2 Lserine-binding aptamer candidates or Hcy10.

\begin{tabular}{|c|c|c|c|c|c|c|c|}
\hline \multirow{2}{*}{ Domain } & \multicolumn{7}{|c|}{ Nucleotide Position in DNA Sequence from 5' end } \\
\hline & Ser.1 & Ser.2 & Ran.1 & Ran.2 & Red.1 & Red.2 & Hcy10 \\
\hline AGTGGA & --- & --- & --- & 27 & --- & --- & 46 \\
\hline AGTG-- & --- & --- & $27,88^{*}$ & $27,88^{*}$ & $27,88 *$ & $35,88^{*}$ & $46,88^{*}$ \\
\hline AGCACA & $4 *, 53$ & $4 *$ & 47 & --- & 47 & 54 & --- \\
\hline GTGC & $28,68^{*}$ & $68 *$ & $28,89 *$ & $89 *$ & $28,89 *$ & $36,89^{*}$ & $89 *$ \\
\hline TACC & $32,72 *$ & $41,72 *$ & $2^{*}$ & $2 *$ & $2 *$ & $2 *$ & $2 *$ \\
\hline АTCT & --- & 23 & $33,94 *$ & $38,94 *$ & $33,94 *$ & $41,94 *$ & $94 *$ \\
\hline GATA & --- & 28,40 & $20,81^{*}$ & $31,55,80^{*}$ & $20,81 *$ & $28,81^{*}$ & $81 *$ \\
\hline TATT & --- & 30 & $10^{*}$ & $10^{*}$ & $10^{*}$ & $10^{*}$ & $10 *$ \\
\hline CGTG & $\begin{array}{c}27,35,67^{*} \\
75^{*}\end{array}$ & $67^{*}, 75^{*}$ & --- & 46,73 & --- & 22 & 74 \\
\hline GTGA & $36,76^{*}$ & $76^{*}$ & --- & 47,51 & --- & --- & 33 \\
\hline ACCG & $33,41,73 *$ & $73 *$ & --- & --- & --- & 76 & --- \\
\hline
\end{tabular}

*motif present in primer-binding region 
Many shorter sequence motifs were common among several aptamer candidates. Table 2.12 shows the common binding motifs that were generated from the random regions of the SELEX libraries. Hcy10 was shown to have some affinity for L-serine (section 2.5.2), and there were a few shared sequence motifs between Hcyl0 and the other L-serine-binding aptamer candidates. Motifs GAAG, and TGAT for example are shared between Hcy10 and many other candidates, and could be implicated in L-serine-binding. Since the goal of these SELEX experiments was to find an L-serine-binding aptamer with improved L-serine-binding ability over Hcy10, motifs shared by the aptamer candidates but not seen in Hcy10 were of interest. For example, motif GATC was identified in all aptamer candidates and was not present in Hcy10. As this motif was selected in each of the three SELEX experiments, it could be implicated in Lserine binding. It has also been suggested that genetic coding triplets could serve as naturally occurring conserved motifs identified within amino acid-binding sequences. ${ }^{159,160,170,173,278,279}$ An examination of RNA sequences with affinity for amino acids found a correlation to the presence of cognate coding triplets within the binding sites. ${ }^{278}$ The presence of DNA codons which encode L-serine was examined in the six L-serine aptamer candidates. The presence of DNA codons which encoded L-serine was elevated compared to all other amino acid codons, shown in Table 7.2. Future work could include an investigation into the prevalence of these coding triplets throughout the selections to determine if a significant correlation exists.

When SELEX libraries are designed, primer-binding regions are included on the 5' and 3' ends to allow for PCR amplification following each selection round. These are known sequences that are not always involved in the target-binding interaction. ${ }^{280}$ Since Table 2.10 showed reverse primer-binding sequences had been repeated in sequences Ser.1 (Original SELEX), Ran.1 (Randomized SELEX), Red.1 and Red.2 (Redirected SELEX), the primer- 
binding sequences could be involved in L-serine-binding. Motifs identified from the primer binding regions that had been repeated throughout the aptamer candidates are shown in Table 2.13. The 6-nucleotide motif AGCACA was present in the forward primer-binding region in the Original SELEX library, and the motif was repeated within the sequence of Ser.1 from this same selection, as well as appearing within the sequences Ran.1 (Randomized SELEX), Red.1, and Red.2 (Redirected SELEX) from different selection experiments which used different primers. The shorter motif GATA, found in the reverse primer-binding site of the Randomized and Redirected SELEX libraries, was repeated once in Ran.1 (Randomized SELEX), Red.1 and Red.2 (Redirected SELEX), and repeated twice within the sequence Ran.2 (Randomized SELEX). This motif also appeared twice within the aptamer candidate Ser.2 (Original SELEX) which used a different set of primers. The appearance of common motifs from the primerbinding regions within aptamer candidates from each SELEX experiment indicated a likely interaction between the primer-binding sequences and the target L-serine.

\subsubsection{Comparison of SELEX experiments}

In the selection of L-serine-binding aptamers, three separate starting libraries were used and three SELEX experiments were performed in parallel. The output of each selection experiment was compared to gain insight on the effectiveness of each selection strategy to generate promising aptamer candidates. The trends observed from the comparison of each selection experiment are summarized in Table 2.14. The trends observed in common sequence motifs among the resulting aptamer candidates are displayed in Table 2.15. 
Table 2.14: Trends observed when comparing the Original SELEX, Randomized SELEX, and Redirected SELEX experiments.

\begin{tabular}{|c|c|c|c|c|c|c|}
\hline & \multicolumn{2}{|c|}{$\begin{array}{l}\text { Original Pool } \\
\text { SELEX }\end{array}$} & \multicolumn{2}{|c|}{$\begin{array}{c}\text { Randomized Pool } \\
\text { SELEX }\end{array}$} & \multicolumn{2}{|c|}{$\begin{array}{l}\text { Redirected Pool } \\
\text { SELEX }\end{array}$} \\
\hline $\begin{array}{c}\text { Number of Selection } \\
\text { Rounds }\end{array}$ & \multicolumn{2}{|c|}{11} & \multicolumn{2}{|c|}{8} & \multicolumn{2}{|c|}{ 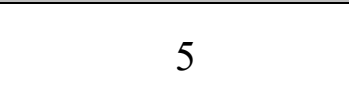 } \\
\hline $\begin{array}{l}\text { Stringent Selection } \\
\text { Rounds }\end{array}$ & \multicolumn{2}{|c|}{2} & \multicolumn{2}{|c|}{5} & \multicolumn{2}{|c|}{2} \\
\hline $\begin{array}{c}\text { Diversity of Starting } \\
\text { Library }\end{array}$ & \multicolumn{2}{|c|}{$\begin{array}{c}\text { Most diverse } \\
(99.92 \%)\end{array}$} & \multicolumn{2}{|c|}{$\begin{array}{l}\text { Moderately diverse } \\
(95.58 \%)\end{array}$} & \multicolumn{2}{|c|}{$\begin{array}{l}\text { Least diverse } \\
(54.00 \%)\end{array}$} \\
\hline $\begin{array}{c}\text { Diversity of Final } \\
\text { Round }\end{array}$ & \multicolumn{2}{|c|}{$\begin{array}{l}\text { Most diverse } \\
(88.69 \%)\end{array}$} & \multicolumn{2}{|c|}{$\begin{array}{c}\text { Moderately diverse } \\
(59.91 \%)\end{array}$} & \multicolumn{2}{|c|}{$\begin{array}{c}\text { Least diverse } \\
(53.80 \%)\end{array}$} \\
\hline $\begin{array}{l}\text { Enrichment over } \\
\text { Selection }\end{array}$ & \multicolumn{2}{|c|}{$\begin{array}{l}\text { Most enrichment } \\
(0.08 \% \text { to } 7.01 \%)\end{array}$} & \multicolumn{2}{|c|}{$\begin{array}{c}\text { Moderate enrichment } \\
(1.56 \% \text { to } 6.49 \%)\end{array}$} & \multicolumn{2}{|c|}{$\begin{array}{c}\text { Least enrichment } \\
(11.63 \% \text { to } 13.26 \%)\end{array}$} \\
\hline $\begin{array}{l}\text { Change in base } \\
\text { distribution of } \\
\text { library }\end{array}$ & \multicolumn{2}{|c|}{$\begin{array}{c}\text { Increase in } \mathrm{A} \\
\text { Decrease in } \mathrm{T}, \mathrm{C}, \mathrm{G}\end{array}$} & \multicolumn{2}{|c|}{$\begin{array}{l}\text { Increase in } A \\
\text { Decrease in } G\end{array}$} & \multicolumn{2}{|c|}{ No Change } \\
\hline $\begin{array}{c}\text { Hcy10 sequence } \\
\text { identified in library }\end{array}$ & \multicolumn{2}{|c|}{ No } & \multicolumn{2}{|c|}{ Yes } & \multicolumn{2}{|c|}{ Yes } \\
\hline $\begin{array}{c}\text { Enriched in soil } \\
\text { solution }(\mathbf{Y} / \mathbf{N})\end{array}$ & $\begin{array}{l}\text { Ser.1 } \\
\text { Yes }\end{array}$ & $\begin{array}{l}\text { Ser.2 } \\
\text { No }\end{array}$ & $\begin{array}{c}\text { Ran.1 } \\
\text { Yes }\end{array}$ & $\begin{array}{c}\text { Ran.2 } \\
\text { Yes }\end{array}$ & $\begin{array}{c}\text { Red.1 } \\
\text { Yes }\end{array}$ & $\begin{array}{c}\text { Red.2 } \\
\text { No }\end{array}$ \\
\hline
\end{tabular}


Table 2.15: Trends observed in the presence of common sequence motifs in each L-serine-binding aptamer candidate.

\begin{tabular}{|c|c|c|c|c|c|c|}
\hline \multirow{2}{*}{ Motif } & \multicolumn{2}{|c|}{ Original SELEX } & \multicolumn{2}{c|}{ Randomized SELEX } & \multicolumn{2}{c|}{ Redirected SELEX } \\
\cline { 2 - 6 } & Ser.1 & Ser.2 & Ran.1 & Ran.2 & Red.1 & Red.2 \\
\hline $\begin{array}{c}\text { Reverse Primer-binding region } \\
\text { repeated }\end{array}$ & Yes & No & Yes & No & Yes & Yes \\
\hline $\begin{array}{c}\text { 37 nucleotide motif (5'- } \\
\text { GGAAGAGCACACGTCTGAA }\end{array}$ & No & No & Yes & No & Yes & Yes \\
\hline $\begin{array}{c}\text { CTCCAGTCACTTAGGCATC- } \\
\text { 3') }\end{array}$ & Nes & No & Yes & No & Yes & Yes \\
\hline $\begin{array}{c}\text { 13 nucleotide motif (5'- } \\
\text { GGAGAGCACACG-3') }\end{array}$ & Nes & Yes & Yes & Yes & Yes & Yes \\
\hline 4 nucleotide motif(5'-GATC-3') & Yes & No \\
\hline
\end{tabular}


Table 2.14 displays some interesting trends observed in the diversity and enrichment of the three SELEX libraries. The highest diversity was found in the Original SELEX starting library, which was expected as this library had never been used in a selection and should have contained no sequence bias. The least diversity was seen in the Redirected SELEX starting library, which demonstrated how this library had already been enriched towards L-homocysteine prior to the library's use in SELEX for L-serine. Despite the low diversity in this starting library, the presence of a higher proportion of L-homocysteine-binding sequences could have translated to more sequences with affinity for similar small molecule, L-serine, present in the Redirected SELEX starting library. When the sequence diversity of the final selection rounds was compared to the starting libraries, the largest change in sequence diversity was observed for the Randomized SELEX. This could indicate an enrichment of particular sequences towards L-serine over the course of the selection. This Randomized SELEX was also subjected to the most number of stringent selection rounds, which could have contributed to large change in sequence diversity as non-binding sequences were eliminated. The largest change in enrichment was observed in the Original SELEX, as the starting library had an enrichment of nearly zero, and rose to $7.01 \%$. However, the Redirected SELEX reached the highest level of enrichment, at $13.26 \%$, because it started with a proportion of its starting library already enriched towards Lhomocysteine, with a starting enrichment value of $11.63 \%$. Regardless of the diversity of the starting libraries, all selections showed enrichment towards L-serine, and generated aptamer candidates showing enrichment for L-serine in soil solution.

Table 2.15 displays the most common sequence motifs found in the selected L-serine aptamer candidates that were not present in Hcy10, which could be important for L-serinebinding. Four of the aptamer candidates had their reverse primer-binding site present within their 
randomized regions. These regions were likely inserted during PCR, but were maintained in the library throughout the SELEX experiments. Three aptamer candidates had extensive sequence homology, with the long 37-nucleotide conserved region 5'-

GGAAGAGCACACGTCTGAACTCCAGTCACTTAGGCATC-3'. A shorter 13-nucleotide section of this domain 5' -GGAAGAGCACACG-3' was present in four candidates, while the short motif 5'-GATC-3' was present in all selected L-serine aptamer candidates. As these conserved domains were generated from multiple starting libraries, they could be important for L-serine binding.

\subsection{Conclusions}

In this chapter, DNA aptamers binding crop exudate L-serine were developed. An existing aptamer for L-homocysteine was evaluated for L-serine binding, and was found to bind immobilized L-serine with an apparent $\mathrm{K}_{\mathrm{d}} \sim 1 \mu \mathrm{M}$. Microscale thermophoretic analysis revealed that Hcy 10 did not bind L-serine in solution, which excluded it as a candidate for a smart fertilizer application.

Novel L-serine-binding aptamers were developed using SELEX from three separate starting libraries: Original SELEX; Redirected SELEX which was derived from an Lhomocysteine selection; and Randomized SELEX which was derived from the Hcy10 aptamer. The Original, Randomized, and Redirected SELEX experiments underwent 11, 8, and 5 selection rounds, respectively. Sequences obtained by the final selection rounds had withstood increased stringency through decreased target concentration, decreased target incubation time, selection in soil solution, as well as counter selections against L-glycine and L-threonine. High throughput sequencing and analysis using AptaCluster identified sequences most enriched in soil solution 
and in buffer from each SELEX library, resulting in six aptamer candidates for further characterization: Ser.1, Ser.2, Ran.1, Ran.2, Red.1, and Red.2.

Analysis of each SELEX experiment showed that using an original library provided the most sequence diversity, as well as the biggest change in enrichment over the selection. Using a randomized aptamer library showed the biggest change in sequence diversity. Using the final rounds from a previous L-homocysteine selection as the starting library provided the least diversity in the starting pool, but resulted in the highest level of enrichment. Considerable sequence homology was found among aptamer candidates from each SELEX experiment, which provided possible L-serine binding motifs for further study. Each SELEX strategy, combined with high-throughput sequencing, allowed the identification of L-serine-binding aptamer candidates enriched in soil solution which can now be further characterized for use in a smart fertilizer system. 
3 Characterization of L-serine-binding aptamers 


\subsection{Chapter Objectives}

In this chapter, aptamer candidates generated from the three SELEX experiments in Chapter 2 were studied to find the aptamers with the best affinity for L-serine when L-serine was either immobilized or free in solution. Minimer aptamers were rationally designed and characterized to find an optimized aptamer candidate for use in fertilizer applications. From one of these aptamer candidates, a Spiegelmer was synthesized to investigate the aptamer-target binding interactions, as well as to increase nuclease resistance.

\subsection{Statement of Contributions}

Sepharose bead assays were performed by E. Mastronardi and K. Cyr, and analyzed by E. Mastronardi. Microscale thermophoresis experiments were run by 2 bind in Germany. All other experiments were run and analyzed by E. Mastronardi. 


\subsection{Introduction}

\subsubsection{Measuring aptamer binding affinity $\left(K_{d}\right)$}

Characterizing aptamer candidates is often the bottleneck in the aptamer development pipeline. Once SELEX experiments are completed, numerous aptamer candidates are generated that need to be characterized. Advanced technology like high-throughput sequencing analysis of aptamer libraries can generate millions of sequences, while characterization methods are generally low-throughput, with the limiting step being that each sequence needs to be processed individually. ${ }^{281}$ Often fewer than ten aptamer candidates from a SELEX experiment are synthesized and characterized. These practical limitations emphasize the importance of starting the characterization process with promising aptamer candidates. ${ }^{281}$

The most common parameter with which aptamers are characterized is by their dissociation constant $\left(\mathrm{K}_{\mathrm{d}}\right)$. This is a numerical value reported as a molar concentration that describes an aptamer's interaction with its target molecule in equilibrium conditions, when the binding of the aptamer to the target is $1: 1$. The $\mathrm{K}_{\mathrm{d}}$ is inversely related to the aptamer's affinity for its target, where a small $\mathrm{K}_{\mathrm{d}}$ value represents high binding affinity. To determine the apparent $\mathrm{K}_{\mathrm{d}}$ of an aptamer-target interaction, either the aptamer or target concentration is held constant while titrating an increasing concentration of the other binding partner to generate a binding isotherm. ${ }^{282}$ These measurements often rely on a large mass increase that occurs when an aptamer binds its target, and utilize mass-sensitive or separation-based techniques such as surface plasmon resonance (SPR), quartz crystal microbalance (QCM), high-performance liquid chromatography (HPLC), capillary electrophoresis (CE), or equilibrium dialysis. ${ }^{24}$

Measurements for $\mathrm{K}_{\mathrm{d}}$ can also depend on the target molecule having some intrinsic fluorescence or absorbance, using techniques such as fluorescence intensity, fluorescence polarization, and 
UV-vis absorption..$^{24}$ Aptamers for small molecule targets do not undergo a large change in mass upon target-binding, and small molecules do not always have intrinsic fluorescence/absorbance, making the aforementioned $\mathrm{K}_{d}$ methods a challenge. To measure the $\mathrm{K}_{\mathrm{d}}$ for small molecule targets, the target is often immobilized in methods such as column chromatography or magnetic bead separation, or the target could be labeled to make use of fluorescence methods. ${ }^{24}$

\subsubsection{Challenges in measuring aptamer binding affinity $\left(K_{d}\right)$ for small molecule targets}

The majority of SELEX experiments are performed for protein targets, with only $25 \%$ of selections performed for small molecules. When examining the relation between target type and binding affinity, it was found that small molecules are generally weaker binders when compared to larger target types. ${ }^{18}$ Protein targets offer many more functional groups for aptamer interaction than do small molecule targets which could be one explanation for this deficit in binding affinity seen for small molecules. Though small molecules as a group seem to have weaker binding interactions with aptamers, there are many examples of successful aptamers for small molecule targets. For example, aptamers selected for BPA, oxytetracycline, and aflatoxins B1 and B2 all showed binding affinities in the low nanomolar range. ${ }^{220,283-285}$ Some of the most studied, and widely-used aptamers in literature are for the small molecules ATP, cocaine, and theophylline. ${ }^{24}$ In fact, these aptamers are often used in proof-of-concept studies, which develop novel aptamer-based technology. Technical difficulties related to characterizing aptamers for small molecules could be another explanation for the weaker binding interactions observed for small molecule targets.

Obtaining a reliable dissociation constant can be challenging for small molecule targets. When an aptamer $(>10000 \mathrm{~g} / \mathrm{mol})$ binds a small molecule target $(<1000 \mathrm{~g} / \mathrm{mol})$, there is not a large change in mass observed, making the aptamer-target complex difficult to detect using 
typical mass-sensitive or separation-based techniques. ${ }^{281}$ To circumvent this issue, either the aptamer or target is often immobilized to a solid support. Immobilizing the aptamer could alter its ability to fold, while immobilizing the target could hinder aptamer binding. Common solid supports including agarose/sepharose, and magnetic or acrylic beads are much larger than small molecule targets, often causing significant non-specific binding. Immobilizing the target may also cause the aptamer to preferentially bind the target along with some portion of the solid support. For example, an aptamer for the small molecule dye sulfohordamine B had a $\mathrm{K}_{\mathrm{d}}$ value of $190 \pm 20 \mathrm{nM}$ when the dye was immobilized to agarose, but dropped to $660 \pm 60 \mathrm{nM}$ when the dye was free in solution. ${ }^{248}$ As many applications for aptamers require the detection of molecules in solution, obtaining a representative binding affinity can be a challenge.

Looking at how the type of binding assay used can affect the resulting $\mathrm{K}_{\mathrm{d}}$, McKeague et al. examined multiple aptamers for the small molecule ochratoxin A (OTA, Mw: $403.8 \mathrm{~g} / \mathrm{mol}$ ). ${ }^{281}$ The aptamers were put through a panel of binding assays including equilibrium dialysis, ultrafiltration, affinity chromatography, fluorescence polarization, SPR, DNase I protection assay, SYBR Green displacement assay, and a gold nanoparticle protection assay to examine the effects the chosen assay had on the resulting $K_{d}$. Inconsistencies in the $K_{d}$ s were found for the same aptamer over multiple tests and no aptamer performed well in every test. It was suggested that multiple binding assays should be undertaken when determining the $\mathrm{K}_{d}$ for small molecule targets. As there is no one technique for measuring aptamer binding affinity that is applicable to all target types, measuring the binding affinity of aptamer candidates is often the most time consuming step of aptamer development. 


\subsubsection{Challenges with moving aptamers into application}

\subsubsection{General considerations}

Aptamers offer many advantages such as a low production cost, ease of labelling, and stability that make them attractive affinity ligands for sensors and delivery applications. Moving aptamers from proof-of-concept studies to real world applications can sometimes be challenging. An aptamer's target recognition is highly dependent on the secondary and tertiary structures they form. Immobilizing an aptamer or target, or changing the buffer conditions (buffering agent, cationic composition, $\mathrm{pH}$ ) can affect the aptamer's ability to bind its target, which can impede an aptamer's incorporation into more complex applications. ${ }^{18,281}$ With these challenges in mind at the outset of SELEX experiments, careful planning can minimize the hurdle researchers will face before an aptamer can be applied in a useful system. For example, if an aptamer is desired for use detecting a target in a complex matrix, this complex matrix can be used during the SELEX experiment, ensuring only aptamers capable of binding their target in this complex matrix are kept. Considerations for modifying the selection to reflect the downstream applications could include aptamer/target immobilization, selection matrix, temperature, and interfering molecules for example.

\subsubsection{The development of minimers to increase utility of aptamers}

After a SELEX experiment, each full length aptamer contains nucleotides important for target-binding, nucleotides that provide structural support for the binding site, and nucleotides which are not involved in target-binding. ${ }^{286}$ The longer the aptamer, the larger the production cost for any downstream applications. Truncating unnecessary nucleotides to form a minimal binding aptamer, or "minimer", could ease aptamers into applications. Generating minimers can be done through computational structural analysis of aptamer sequences, through a modified 
DNase footprinting assay, or screening of point mutations in the aptamer's sequence. ${ }^{287,288}$ In addition to reducing cost, removing non-essential nucleotides can sometimes increase the affinity for the target. ${ }^{288,289}$

\subsubsection{Increasing the nuclease resistance of aptamers}

Another challenge to applying aptamers in biological systems is avoiding nuclease degradation. Several strategies exist to overcome these challenges. SELEX experiments have been successful using nucleotides with 2'-amino, 2'-fluoro, and 2'-O-methyl modifications

conferring increased nuclease resistance to the resulting aptamers. ${ }^{290}$ This nuclease resistance can also be achieved by capping the 5' and 3' ends of an aptamer with biotin or inverted bases, or by substituting sulfur for oxygen in the phosphodiester backbone. ${ }^{10,13}$ Modifications such as these could also be useful in preventing the degradation of aptamers in agricultural conditions.

Spiegelmers, a registered trademark of NOXXON Pharma, are mirror-image aptamers that are not recognized by natural nucleases. ${ }^{291}$ Unlike traditional nucleic acids composed of D(deoxy)ribose nucleotides, Spiegelmers are composed of L-(deoxy)ribose nucleotides which do not occur in nature. Naturally occurring nuclease enzymes are stereoselective for Doligonucleotides, allowing Spiegelmers to have prolonged stability. To generate Spiegelmers, traditional D-aptamers are selected for a molecule that is the mirror-image of the target of interest. Once an aptamer is selected, it can be synthesized using L-nucleotides and will bind the target of interest. ${ }^{291}$ Using Spiegelmers can improve aptamer stability while minimizing the need for more complex modifications. 


\subsection{Materials and Methods}

\subsubsection{Chemicals and Reagents}

DNA synthesis was carried out on a BioAutomation Corporation MerMade 6 oligonucleotide synthesizer using standard phosphoramidite chemistry. All amidites, modifiers, and reagents were obtained from Glen Research, $1000 \AA$ CPG $1 \mu$ mol scale columns were obtained from BioAutomation, and synthesis grade acetonitrile was obtained from VWR. 28\% ammonium hydroxide solution was obtained from Sigma-Aldrich (Oakville, ON, Canada). For Spiegelmer synthesis, the following L-Amidites: Beta-L-deoxy Adenosine (n-bz) CED phosphoramidite, Beta-L-deoxy Cytidine (n-acetyl) CED phosphoramidite, Beta-L-deoxy Guanosine (n-ibu) CED phosphoramidite, Beta-L-Thymidine CED phosphoramidite, as well as beta-L deoxy Cytidine (N-Ac) 3'-lcaa CPG 1000A support columns, were ordered from ChemGenes.

For affinity experiments, NHS-activated Sepharose 4 Fast Flow, ninhydrin, and buffer components: sodium bicarbonate $\left(\mathrm{NaHCO}_{3}\right)$, sodium acetate $\left(\mathrm{CH}_{3} \mathrm{COONa}\right)$, sodium phosphate dibasic $\left(\mathrm{Na}_{2} \mathrm{HPO}_{4}\right)$, potassium phosphate monobasic $\left(\mathrm{KH}_{2} \mathrm{PO}_{4}\right)$, magnesium chloride $\left(\mathrm{MgCl}_{2}\right)$, and $\mathrm{HCl}$ were obtained from Sigma-Aldrich (Oakville, ON, Canada). Additional buffer components, sodium chloride $(\mathrm{NaCl})$, Tris, and urea, as well as target molecules, L-serine, Lglycine, and L-threonine were obtained from BioShop Canada (Burlington, ON, Canada). Costar Spin-X cellulose acetate centrifuge filters were obtained from Corning Incorporated (New York, United States).

For gold nanoparticle synthesis, hydrocholoric acid $(\mathrm{HCl})$, nitric acid $\left(\mathrm{HNO}_{3}\right)$ were obtained from VWR. Gold chloride $\left(\mathrm{HAuCl}_{4}\right)$ and sodium citrate were obtained from SigmaAldrich (Oakville, ON, Canada). 
For the nuclease resistance study, DNase I, and PAGE reagents: acrylamide/bisacrylamide $40 \%$ solution, Tris, boric acid, EDTA and N,N,N',N'-tetramethylethylenediamine (TEMED), were obtained from BioShop Canada (Burlington, ON, Canada), while formamide was obtained from Sigma-Aldrich (Oakville, ON, Canada).

\subsubsection{Structural analysis}

\subsubsection{Computational Structural Analysis}

The predicted structure of each aptamer candidate was determined by entering the DNA sequences into RNAstructure software, selecting DNA, and using the default settings (http://rna.urmc.rochester.edu/RNAstructureWeb/Servers/Predict1/Predict1.html). To identify possible G-quadruplex-forming domains, the aptamer sequences were examined using QGRS mapping software (http://bioinformatics.ramapo.edu/QGRS/index.php).

\subsubsection{Melting Temperature Analysis}

The melting temperature of each aptamer was studied using variable temperature UV-Vis spectroscopy, using a Varian Cary 300 Bio UV-Vis spectrophotometer with a $6 \times 6$ Peltier thermostatted multicell holder. Aptamers to be tested were diluted in modified PBS (10 mM $\mathrm{Na}_{2} \mathrm{HPO}_{4}, 1.8 \mathrm{mM} \mathrm{KH}_{2} \mathrm{PO}_{4}, 5 \mathrm{mM} \mathrm{MgCl}, 490 \mathrm{mM} \mathrm{NaCl}$, $\mathrm{pH}$ 7.4) to a concentration leading to an absorbance value around $0.8 .3 \mathrm{~mL}$ of each aptamer solution were placed in cuvettes with a stir-bar and lid. Using the thermal program, the absorbance at $260 \mathrm{~nm}$ was measured over three temperature cycles (Ramp $180^{\circ} \mathrm{C}-20^{\circ} \mathrm{C}$; Ramp $220^{\circ} \mathrm{C}-80^{\circ} \mathrm{C}$; Ramp $380^{\circ} \mathrm{C}-20^{\circ} \mathrm{C}$ ) with a ramp rate of $1{ }^{\circ} \mathrm{C} / \mathrm{min}$. This thermal denaturation experiment was performed with and without $200 \mu \mathrm{M}$ L-serine present, and was repeated at $295 \mathrm{~nm}$ to look for G-quadruplexes. The melting temperatures were determined by plotting the absorbance values at each temperature range and using the standard curves analysis feature of SigmaPlot. 


\subsubsection{Determining Aptamer Affinity}

\subsubsection{Measuring aptamer binding immobilized L-serine using L-serine-conjugated sepharose}

Aptamer candidates were synthesized as described in section 2.4 .5 with a 5 'fluorescein modification, PAGE-purified and confirmed by ESI/LC/MS. Aptamer candidates were diluted in PBS buffer over a range of concentrations ( $2 \mu \mathrm{M}-50 \mathrm{nM}$ for example). L-serine-coupled beads (see section 2.4.2) were diluted 1:1 in PBS buffer, and $20 \mu \mathrm{L}$ of the bead slurry were added to $0.22 \mu \mathrm{m}$ Costar Spin-X cellulose acetate centrifuge filters (Corning Incorporated), for each aptamer concentration to be tested. The beads were washed by adding $100 \mu \mathrm{L}$ of PBS buffer, and centrifuging at $13000 \times \mathrm{g}$. The DNA was heated at $90^{\circ} \mathrm{C}$ for 5 minutes, cooled at $4^{\circ} \mathrm{C}$ for 10 minutes, and left at room temperature for 15 minutes. $100 \mu \mathrm{L}$ of each DNA concentration were added to L-serine-coupled beads, and placed on a shaker at room temperature for 45 minutes. Unbound DNA was removed by centrifuging at $5000 \times \mathrm{g}$ for 1 minute, followed by 3 washes using $100 \mu \mathrm{L}$ of PBS buffer each. For elution of bound DNA, $100 \mu \mathrm{L}$ of $7 \mathrm{M}$ urea was added to the beads, vortexed, and incubated at $90^{\circ} \mathrm{C}$ for 10 minutes. The eluate was collected after centrifugation at $13000 \times \mathrm{g}$ for 1 minute. This was repeated for 3 elutions per test concentration. The fluorescence intensity of the elutions was measured using a Fluorolog Fluorescence Spectrophotometer (Horiba Jobin Yvon, USA) with an excitation wavelength of $490 \mathrm{~nm}$, emission wavelength of $520 \mathrm{~nm}$, and slit widths $5 \mathrm{~nm}$. The amount of DNA eluted at each concentration was calculated by comparing to a standard curve of fluorescence intensity at known concentrations. 


\subsubsection{Measuring aptamer binding L-serine in solution using microscale thermophoresis}

The aptamer and target samples were prepared in house, and sent to 2bind in Germany for microscale thermophoresis analysis. Aptamer candidates were synthesized as described (section 2.4.5) with a 5'fluorescein modification. Minimers tested had a 5' Cy5 modification instead of 5' fluorescein, and were ordered from IDT. Each aptamer sample was $10 \mu \mathrm{M}$ in PBS buffer and $10 \mu \mathrm{L}$ were sent to 2 bind. The target samples sent were $1 \mathrm{~mL}$ of $10 \mathrm{mM}$ L-serine solution in the same buffer. A range of L-serine concentrations (7.6 nM - 250 $\mu \mathrm{M}$ in buffer) was incubated with $20 \mathrm{nM}$ of labeled aptamer and analyzed on a Monolith NT at $25^{\circ} \mathrm{C}$, with $15 \%$ LED power and $80 \%$ laser power.

\subsubsection{Minimer determination}

Minimers were designed from the Red.1 full length sequence. The predicted structure was used to separate the Red.1 sequence into smaller secondary structures. Minimer sequences are shown in Table 3.1. Each minimer sequence was ordered from IDT, PAGE-purified with mass confirmation by mass spectrometry, and sent for microscale thermophoresis analysis as described above.

Table 3.1: Red.1 minimer sequences ordered from IDT.

\begin{tabular}{|l|c|l|}
\hline Name & $\begin{array}{l}\text { Length } \\
\text { (bases) }\end{array}$ & Sequence \\
\hline 5'Loop & 47 & $\begin{array}{l}\text { ATACCAGCTTATTCAATTAGATAGTAAGTGCAATCTAGATCG } \\
\text { GAAGA }\end{array}$ \\
\hline 3'Loop & 50 & $\begin{array}{l}\text { GCACACGTCTGAACTCCAGTCACTTAGGCATCAGATAGTAAG } \\
\text { TGCAATCT }\end{array}$ \\
\hline $\begin{array}{l}\text { Random } \\
\text { region }\end{array}$ & 61 & $\begin{array}{l}\text { AGATAGTAAGTGCAATCTAGATCGGAAGAGCACACGTCTGA } \\
\text { ACTCCAGTCACTTAGGCATC }\end{array}$ \\
\hline $\begin{array}{l}\text { Minus } \\
\text { 3'primer }\end{array}$ & 79 & $\begin{array}{l}\text { ATACCAGCTTATTCAATTAGATAGTAAGTGCAATCTAGATCG } \\
\text { GAAGAGCACACGTCTGAACTCCAGTCACTTAGGCATC }\end{array}$ \\
\hline
\end{tabular}




\subsubsection{Examining aptamer and minimer-target binding using a colorimetric gold nanoparticle (AuNP) test}

\subsubsection{Gold nanoparticle (AuNP) synthesis}

All glassware was washed with aqua regia (3:1 mixture of $\left.\mathrm{HCl} / \mathrm{HNO}_{3}\right)$ for 15 minutes, and thoroughly rinsed with $\mathrm{diH}_{2} \mathrm{O}$. A $100 \mathrm{~mL}$ solution of $1 \mathrm{mM} \mathrm{HAuCl}_{4}$ in $\mathrm{diH}_{2} \mathrm{O}$ was stirred and heated until boiling. Once boiling, a $10 \mathrm{~mL}$ solution of $38.8 \mathrm{mM}$ sodium citrate was quickly added. The suspension changed in colour from pale yellow to dark blue, and finally reaching a wine red colour. This solution was cooled to room temperature, and the synthesis was confirmed by transmission electron microscopy (TEM).

\subsubsection{Analyzing aptamer and minimer-target binding using AuNPs}

The Red.1 aptamer was diluted to $10 \mu \mathrm{M}$ in $\mathrm{diH}_{2} \mathrm{O}$, and $3 \mu \mathrm{L}$ of aptamer were added to $243 \mu \mathrm{L}$ of L-serine (over a range of testing concentrations) and incubated for 30 minutes at room temperature. After incubation, $135 \mu \mathrm{L}$ of $12 \mathrm{nM}$ AuNPs were added, and incubated for an additional 30 minutes. A $72 \mu \mathrm{L}$ solution of $0.75 \mathrm{M} \mathrm{NaCl}$ was added. After 5 minutes, colour change was observed by eye. To test the Random Region Minimer, $3 \mu \mathrm{L}$ of minimer $(10 \mu \mathrm{M}$ in $\mathrm{diH}_{2} \mathrm{O}$ ) was added to $243 \mu \mathrm{L}$ of L-serine (over a range of testing concentrations), and incubated for 45 minutes. Following the minimer-target incubation, $135 \mu \mathrm{L}$ of $12 \mathrm{nM}$ AuNPs were added, and incubated an additional 30 minutes. A $72 \mu \mathrm{L}$ solution of $0.5 \mathrm{M} \mathrm{NaCl}$ was added, and the solutions were left to incubate until a colour change could be observed by eye (1 week). 


\subsubsection{Generating an L-serine-binding Spiegelmer}

\subsubsection{Spiegelmer synthesis and L-serine-binding tests}

A Spiegelmer with sequence: 5'-Cy5-

\section{AGATAGTAAGTGCAATCTAGATCGGAAGAGCACACGTCTGAACTCCAGTCACTTAG}

GCATC-3' was synthesized on a MerMade 6 oligonucleotide synthesizer with L-amidites and columns, and standard reagents. The Spiegelmer was purified using denaturing-PAGE as described in section 2.4.6. The synthesis was confirmed using mass spectroscopy. The binding affinities of the Spiegelmer to L-serine, D-serine, L-glycine, and Tris were determined my microscale thermophoresis as described in section 3.4.3.2.

\subsubsection{Determination of nuclease-resistance}

The random region minimer and corresponding Spiegelmer were diluted to $50 \mu \mathrm{M}$ each in $\mathrm{diH}_{2} \mathrm{O} .10 \mu \mathrm{L}$ of the minimer or Spiegelmer were mixed with $90 \mu \mathrm{L}$ of DNase I reaction buffer (10 mM Tris-HCl, $2.5 \mathrm{mM} \mathrm{MgCl}_{2}, 0.5 \mathrm{mM} \mathrm{CaCl}_{2}, \mathrm{pH} 7.0$ ) for each time point to be tested. To each DNA solution, $1 \mu \mathrm{L}$ of DNase I was added, and the tubes were incubated at $37^{\circ} \mathrm{C}$. For the random region minimer, the DNase I was incubated with the DNA for 0, 30, 60, and 90 seconds. For the Spiegelmer, the DNase I was incubated with the DNA for 0, 12, 36, and 60 hours. The reaction was stopped by adding $1 \mu \mathrm{L} 0.5 \mathrm{M}$ EDTA and incubating tubes at $90^{\circ} \mathrm{C}$

for 10 minutes. The reactions were then analyzed using denaturing PAGE. The protocol listed in section 2.4.6 was followed, however a 19\% gel was used instead of $12 \%$, and run $~ 300$ volts for 2.5 hours. 


\subsection{Results and Discussion}

\subsubsection{Binding affinity determination using L-serine-conjugated sepharose beads}

The binding affinity of an aptamer to its target is described by its dissociation constant $\left(\mathrm{K}_{\mathrm{d}}\right)$. The apparent $\mathrm{K}_{\mathrm{d}}$ of the aptamer candidates were determined for L-serine both immobilized, and in solution. L-serine was immobilized on sepharose beads during the selection of L-serinebinding aptamers, and these L-serine-conjugated sepharose beads were used to screen the aptamer candidates for affinity. A range of aptamer concentrations (50 nM - $2 \mu \mathrm{M})$ were mixed with equal volumes of L-serine-conjugated sepharose beads, and binding DNA was eluted and quantified. A representative binding isotherm for Red.1 is shown in Figure 3.1, with the apparent $K_{d S}$ for each aptamer candidate show in Table 3.2. To examine the selectivity of these aptamer candidates, Tris-conjugated sepharose beads, and L-glycine-conjugated sepharose beads were incubated with a range of aptamer candidate concentrations, and their apparent $\mathrm{K}_{\mathrm{dS}}$ are also displayed in Table 3.2. 


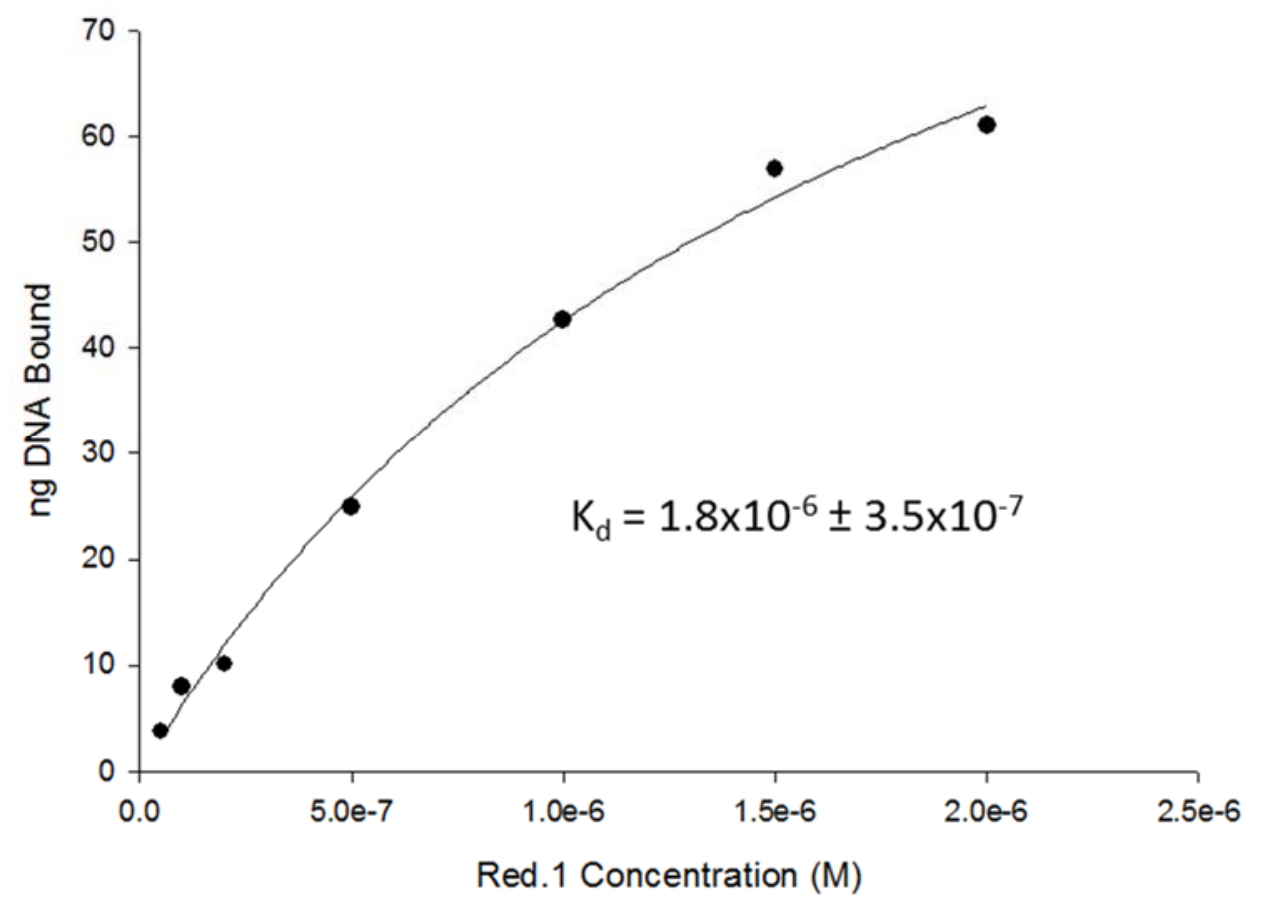

Figure 3.1: A binding isotherm was generated from Red.1 binding L-serine-conjugated sepharose beads. A range of fluorescently modified Red.1 concentrations (50 nM to $2 \mu \mathrm{M})$ was incubated with L-serine-coupled beads, and eluted with excess L-serine and $7 \mathrm{M}$ urea. Fluorescence spectroscopy was used to measure the Red.1 eluted. This binding isotherm was generated using simple ligand-binding in SigmaPlot, and the error is on the fit. 
Table 3.2: Apparent $K_{d} S$ for aptamer candidates determined using sepharose beads conjugated to L-serine, Tris, or L-glycine as target molecules. SigmaPlot simple ligand binding was used to calculate $K_{d} s$, and the error represents the standard deviation between trials $(\mathbf{N}=\mathbf{3})$.

\begin{tabular}{cccc}
\hline \multirow{2}{*}{ Aptamer } & \multicolumn{3}{c}{ Apparent $\mathbf{K}_{\mathbf{d}}(\boldsymbol{\mu M})$} \\
\cline { 2 - 4 } & L-Serine & Tris & L-Glycine \\
\hline Ser.1 & $3.0 \pm 2.7$ & $0.85 \pm 0.68$ & $2.3 \pm 0.13$ \\
Ser.2 & Inconclusive & $2.0 \pm 1.0$ & $4.7 \pm 5.9$ \\
\hline Ran.1 & Inconclusive & Inconclusive & Inconclusive \\
Ran.2 & $2.9 \pm 3.2$ & Inconclusive & No Binding \\
\hline Red.1 & $2.8 \pm 2.0$ & $4.0 \pm 2.7$ & $2.6 \pm 1.3$ \\
Red.2 & $3.5 \pm 2.9$ & $0.72 \pm 0.057$ & Inconclusive \\
\hline
\end{tabular}

*No Binding refers to no trend seen

*Inconclusive refers to a possible $\mathrm{K}_{d}$ value outside the concentrations tested/non-specific binding to sepharose

All aptamer candidates showed binding to immobilized L-serine in the low micromolar range, except for Ser.2 and Ran.1 which showed binding to L-serine but had an apparent $\mathrm{K}_{\mathrm{d}}$ outside the range of test concentrations (Table 3.2). This low micromolar affinity was comparable to other small molecule-binding aptamers, and was superior to most amino acidbinding aptamers shown in Table 2.1. ${ }^{18}$ Red.1 showed the most affinity for immobilized L-serine with an apparent $\mathrm{K}_{\mathrm{d}}$ of $2.8 \pm 2.0 \mu \mathrm{M}$. All of the aptamer candidates showed binding to Tris ranging from 0.85 to $4.0 \mu \mathrm{M}$, except Ran.1, and Ran.2 which showed weak binding outside the range of tested concentrations. Tris was ubiquitous throughout all three SELEX experiments, as it was used as a blocking agent for any activated esters that remained on the sepharose beads after L-serine conjugation, as well as being used as the buffering agent in the SELEX buffer. To mitigate the risk of obtaining aptamers with some affinity to Tris, counter selections against Tris were attempted, as Tris was coupled to the NHS-sepharose resin and used as a control prior to 
every selection round. Despite these counter selections, affinity for Tris remained, and could be due to its structural similarity to L-serine, offering three alkyl hydroxyl groups as binding sites. L-glycine was chosen as a structural control, differing from L-serine by only one alkyl hydroxyl group. Ser.1, Ser.2, and Red.1 showed affinity for L-glycine with apparent $\mathrm{K}_{\mathrm{ds}}$ ranging from 2.3 to $4.7 \mu \mathrm{M}$, while Ran.1 and Red.2 showed weak binding outside the range of tested concentrations (Table 3.2). Ran.2 was the most selective of all the aptamer candidates, showing no binding to L-glycine, and the weakest binding to Tris of all the aptamer candidates (Table $3.2)$.

The small size of the L-serine target compared to the sepharose beads could hinder successful binding measurements. The large sepharose bead and the 6-aminohexanoic acid linker arm provide many possible binding sites for DNA. To ensure non-specific binding of DNA to the sepharose bead or 6-aminohexanoic acid linker was low, a random aptamer of comparable length (81 bases) was incubated with L-serine-conjugated beads as a control, seen in Figure 3.2. 


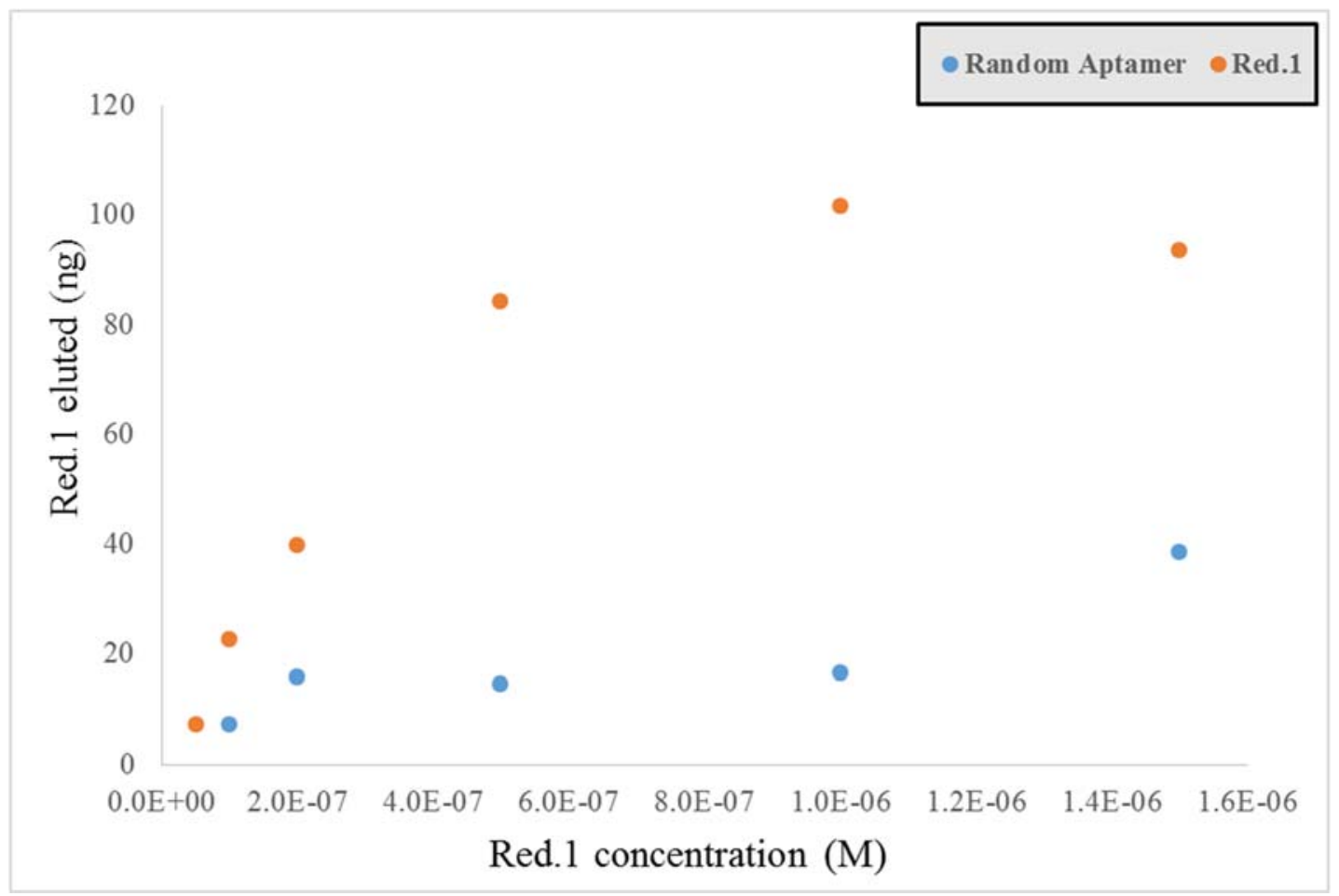

Figure 3.2: Red.1 binding to L-serine-conjugated sepharose compared to a random aptamer control (81 bases). A range of fluorescently modified Red.1 and random aptamer (50 $\mathrm{nM}$ to $1.5 \mu \mathrm{M}$ ) was incubated with L-serine-coupled beads, and eluted with excess Lserine and $7 \mathrm{M}$ urea. Fluorescence spectroscopy was used to measure the DNA eluted.

The 81-base random aptamer had also been selected against an immobilized small molecule target, using epoxide-modified sepharose rather than NHS-sepharose. The random aptamer showed minimal binding to L-serine-conjugated sepharose compared to the Red.1 aptamer that was specific for L-serine, showing the affinity can be attributed to target-binding rather than non-specific binding.

While these affinity tests using immobilized-L-serine were a useful screening method to infer L-serine-binding abilities of these aptamer candidates, it was not possible from these tests to discount the possibility of interference from the solid support. In these tests, the target was immobilized the same way as in the SELEX experiments, so some residual affinity for the solid support or blocking agent could remain. These L-serine-binding aptamers were selected with the 
intent to be used in sensor and smart fertilizer application where the L-serine would be free in soil. With these applications in mind, it was essential to determine the affinities of the aptamer candidates when L-serine was free in solution.

\subsubsection{Binding affinity determination using microscale thermophoresis (MST)}

Measuring the binding affinity of an aptamer for a small molecule target free in solution is a challenge, as target-binding is not accompanied by a large mass increase. Microscale thermophoresis (MST) is a sensitive bioanalytical technique for detecting molecular interactions in solution. This technique relies on the change of thermophoretic mobility that occurs when a binding event alters a molecule's shape, size, charge, or hydration shell. This method can be used to analyze the molecular interactions between proteins, peptides, DNA, sugars, or small molecules. ${ }^{292}$ To determine the apparent binding affinities of these L-serine-binding aptamer candidates in solution, the aptamer candidates were labeled with a fluorophore, mixed with varying L-serine concentrations, and examined using MST by 2bind in Germany. The interactions observed between each aptamer candidate and L-serine in solution are shown in Figure 3.3 and Figure 3.4. 

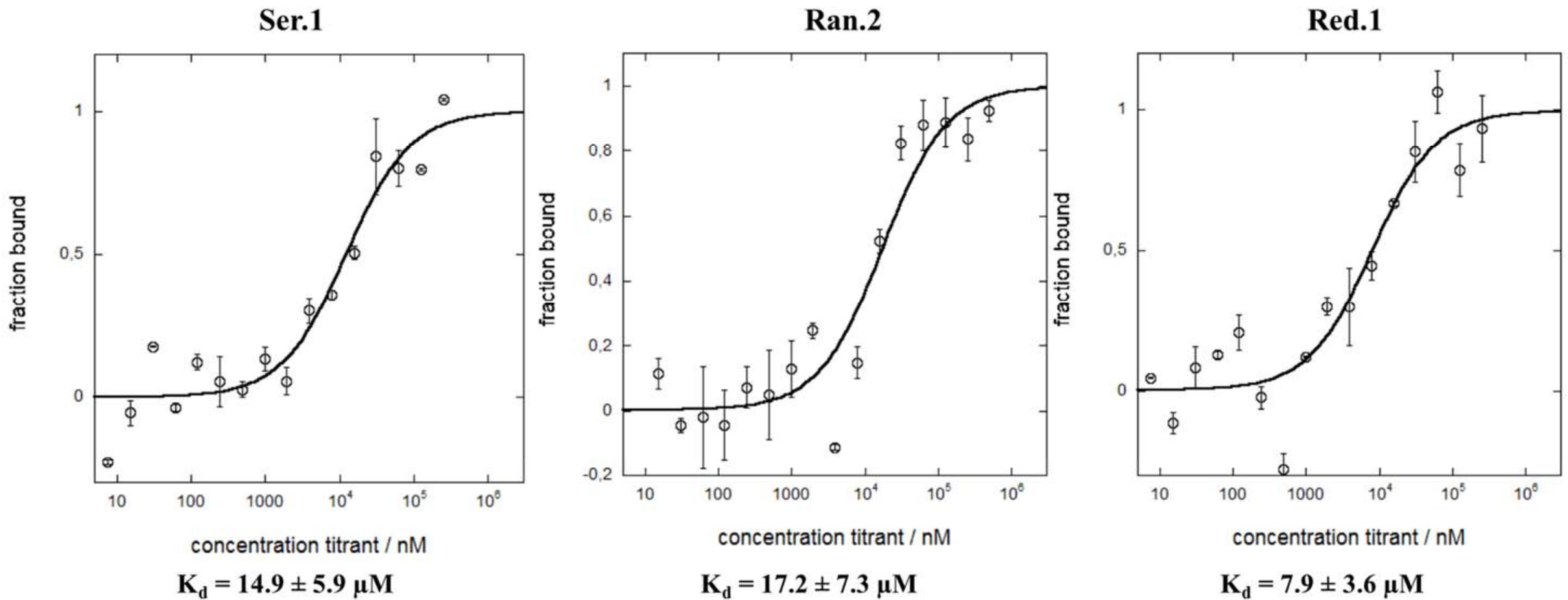

Figure 3.3: Aptamer candidates Ser.1, Ran.2 and Red.1 binding a range of L-serine concentrations in solution, measured by microscale thermophoresis performed by 2 bind. Fluorescently modified DNA $(20 \mathrm{nM})$ was examined after incubation with a range of L-serine concentrations $(7.6 \mathrm{nM}-250 \mu \mathrm{M})$. 

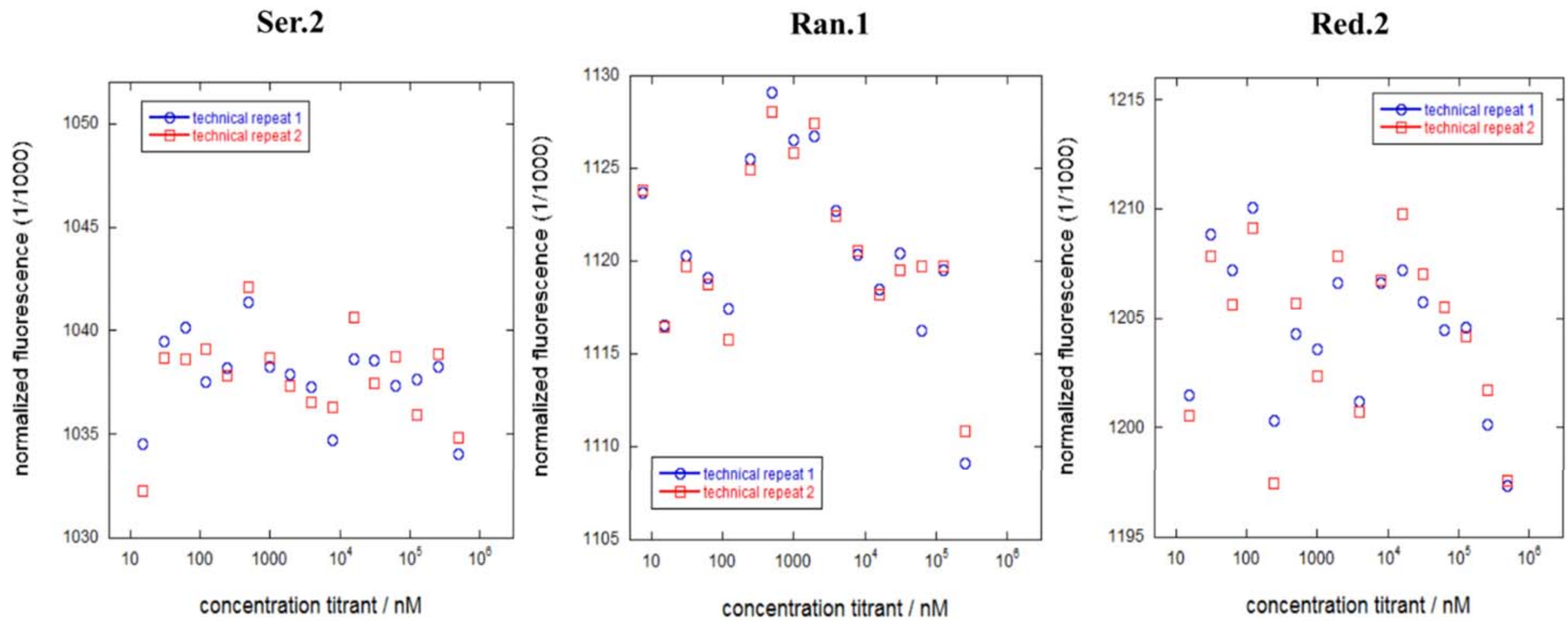

Figure 3.4: Aptamer candidates Ser.2, Ran.1 and Red.2 showed no binding to a range of L-serine concentrations in solution, measured by microscale thermophoresis performed by 2 bind. Fluorescently modified DNA (20 nM) was examined after incubation with a range of $L$-serine concentrations $(7.6 \mathrm{nM}-250 \mu \mathrm{M})$. 
Ser.1, Ran.2, and Red.1 were all able to bind L-serine in solution, with apparent $\mathrm{K}_{\mathrm{d}} \mathrm{S}$ of $14.9 \pm 5.9 \mu \mathrm{M}, 17.2 \pm 7.3 \mu \mathrm{M}$, and $7.9 \pm 3.6 \mu \mathrm{M}$, respectively (Figure 3.3). Of the six aptamer candidates tested, these three aptamers also bound immobilized L-serine most strongly (Table 3.2). The ability of these aptamers to bind immobilized L-serine as well as L-serine in solution showed that the binding interaction to L-serine was not dependent on, or hindered by the solid support. Ser.2, Ran.1, and Red.2 were unable to bind L-serine in solution, seen in Figure 3.4. Ser.2 and Red.2 both bound Tris more strongly than L-serine in the sepharose bead tests (Table 3.2) and since they did not bind L-serine in solution, it is likely these aptamers had affinity for the blocking agent Tris rather than the target, L-serine. Ran.1 showed only weak binding to Lserine during the sepharose bead tests, and can likely be attributed to non-specific interaction with the solid support, as no binding was seen to L-serine in solution. A summary of the aptamer candidates' interaction with L-serine in each affinity test is shown in Table 3.3.

Table 3.3: A summary of L-serine-binding observed by each aptamer candidate using Lserine-conjugated sepharose and microscale thermophoresis.

\begin{tabular}{cccc}
\hline $\begin{array}{c}\text { Aptamer } \\
\text { Candidate }\end{array}$ & $\begin{array}{c}\text { L-serine- } \\
\text { sepharose }\end{array}$ & MST & Conclusions \\
\hline Ser.1 & + & + & Binds L-serine \\
Ser.2 & Inconclusive & - & May have affinity for solid support \\
Ran.1 & Inconclusive & - & May have affinity for solid support \\
Ran.2 & + & + & Binds L-serine \\
Red.1 & + & + & Binds L-serine \\
\hline Red.2 & + & - & May have affinity for solid support \\
\hline
\end{tabular}




\subsubsection{Comparison of aptamer candidates generated from each SELEX strategy}

Each of the three SELEX experiments performed for L-serine generated one aptamer candidate that bound only to immobilized L-serine, and one candidate that bound both immobilized and free L-serine. This finding suggested that all three SELEX strategies employed herein could be useful, as L-serine-binding aptamers with micromolar affinity were generated from an original library, from a redirected L-homocysteine selection, and from a randomized Lhomocysteine aptamer library. The aptamer Red.1, from the redirected L-homocysteine SELEX library, showed the most affinity for L-serine in both the sepharose bead test and microscale thermophoresis, with apparent $\mathrm{K}_{\mathrm{d}}$ of $2.8 \pm 2.0 \mu \mathrm{M}$ and $7.9 \pm 3.6 \mu \mathrm{M}$, respectively. The ability to reuse libraries after several rounds of SELEX for similar molecules could save researchers time, as well as produce high quality aptamers. The Ran.2 aptamer from the randomized aptamer library may provide the most selectivity, as it didn't appear to bind to Tris strongly, or to Lglycine in the sepharose tests (Table 3.2). These results underscore the importance of performing negative selections in SELEX for small molecules, as both the randomized SELEX pool and the redirected SELEX pool had undergone several negative selections against sepharose beads before beginning the L-serine SELEX. These extra negative selections could have contributed to the selection of aptamers with higher affinity and selectivity.

\subsubsection{Structural Analysis for minimer design}

An aptamer's interactions with a target molecule depend upon the aptamer's structure. Predicting an aptamer's structure can help elucidate motifs important for target-binding, give an indication of aptamer stability, and be used to predict minimers or point mutations to increase binding affinity. RNAstructure is a software developed to predict the secondary structures of oligonucleotide sequences using thermodynamics. ${ }^{293}$ This software was used to predict the 
structures of each aptamer candidate and these predicted structures are shown in Figure 3.5 to

Figure 3.10. 

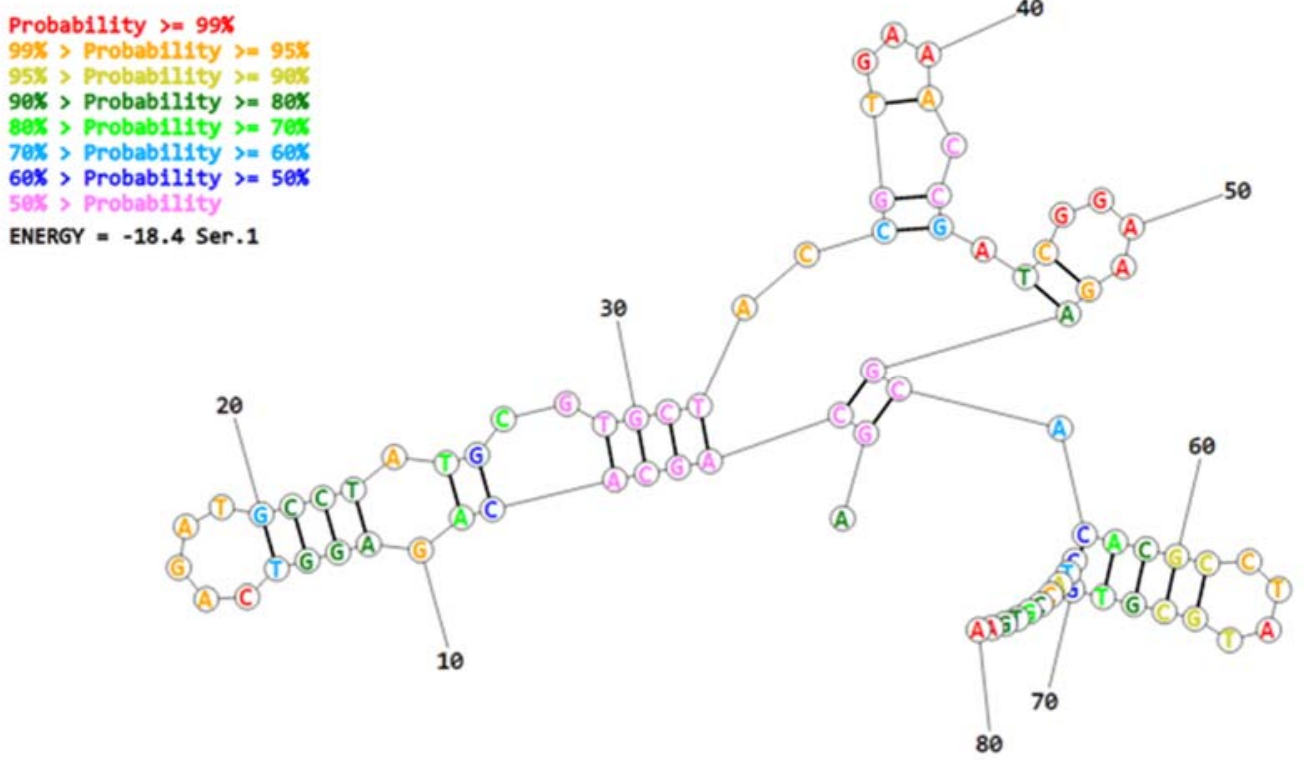

Figure 3.5: The secondary structure prediction for Ser.1 generated by RNAstructure software. The colour represents the probability of each base pair as a percent.
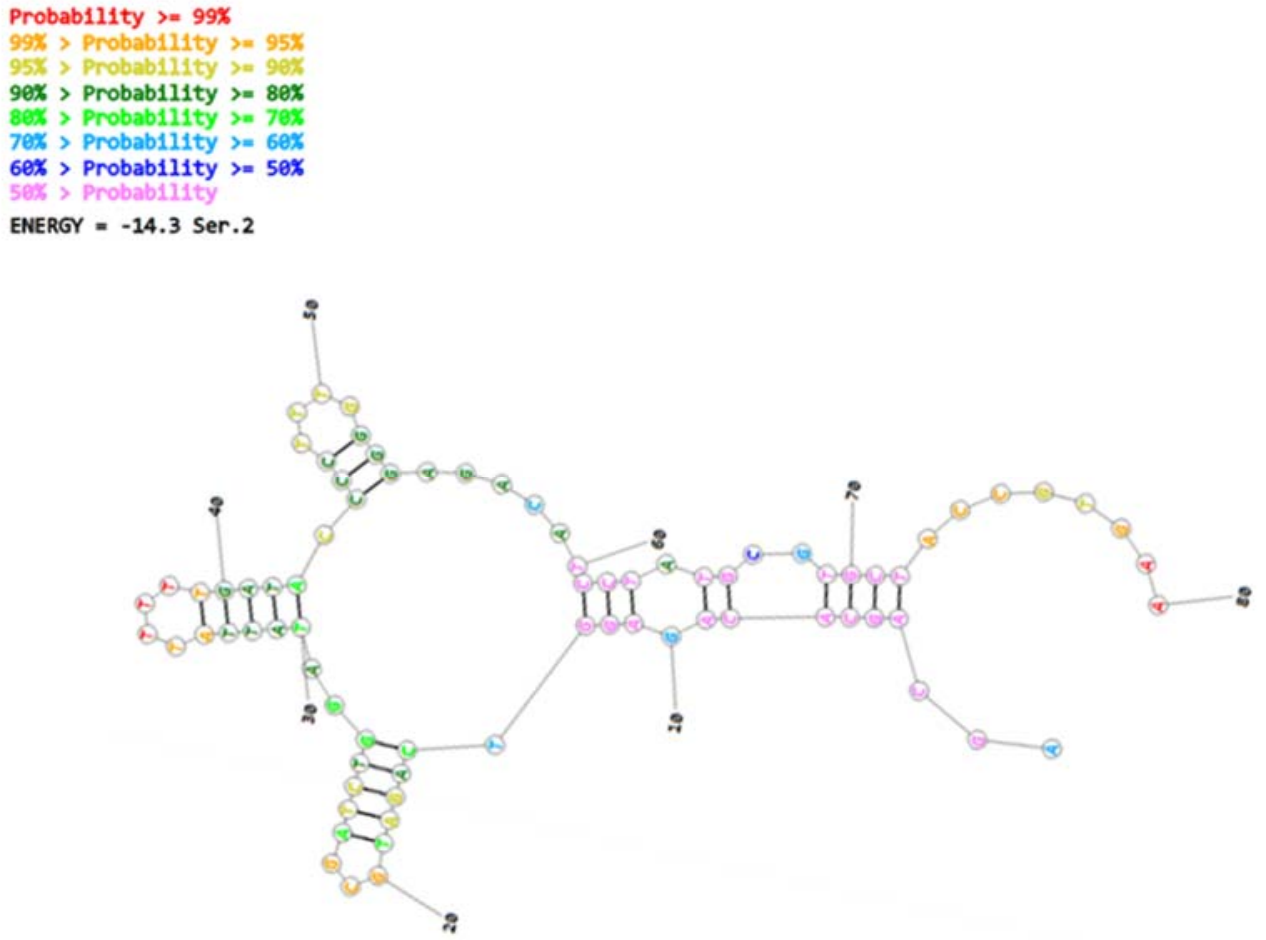

Figure 3.6: The secondary structure prediction for Ser.2 generated by RNAstructure software. The colour represents the probability of each base pair as a percent. 


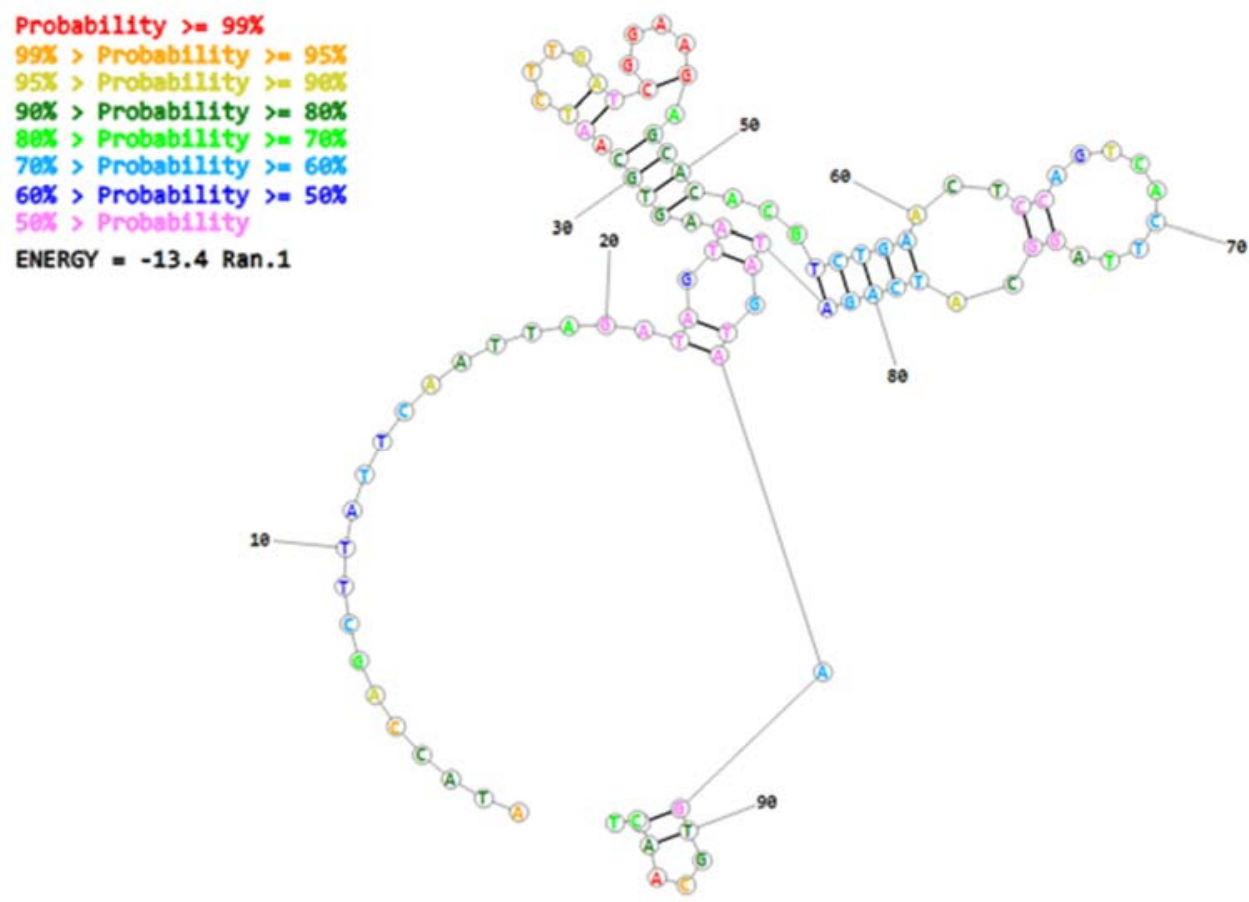

Figure 3.7: The secondary structure prediction for Ran.1 generated by RNAstructure software. The colour represents the probability of each base pair as a percent.

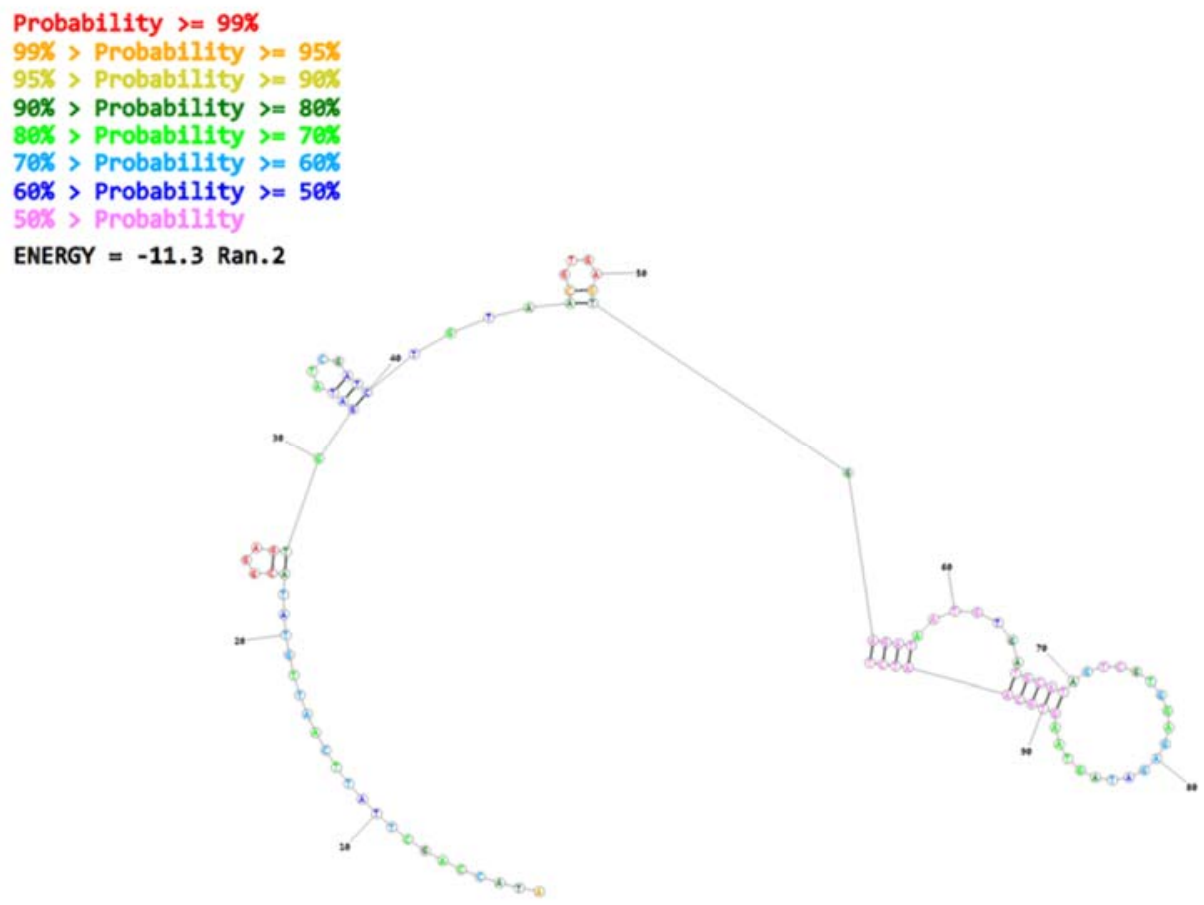

Figure 3.8: The secondary structure prediction for Ran.2 generated by RNAstructure software. The colour represents the probability of each base pair as a percent. 


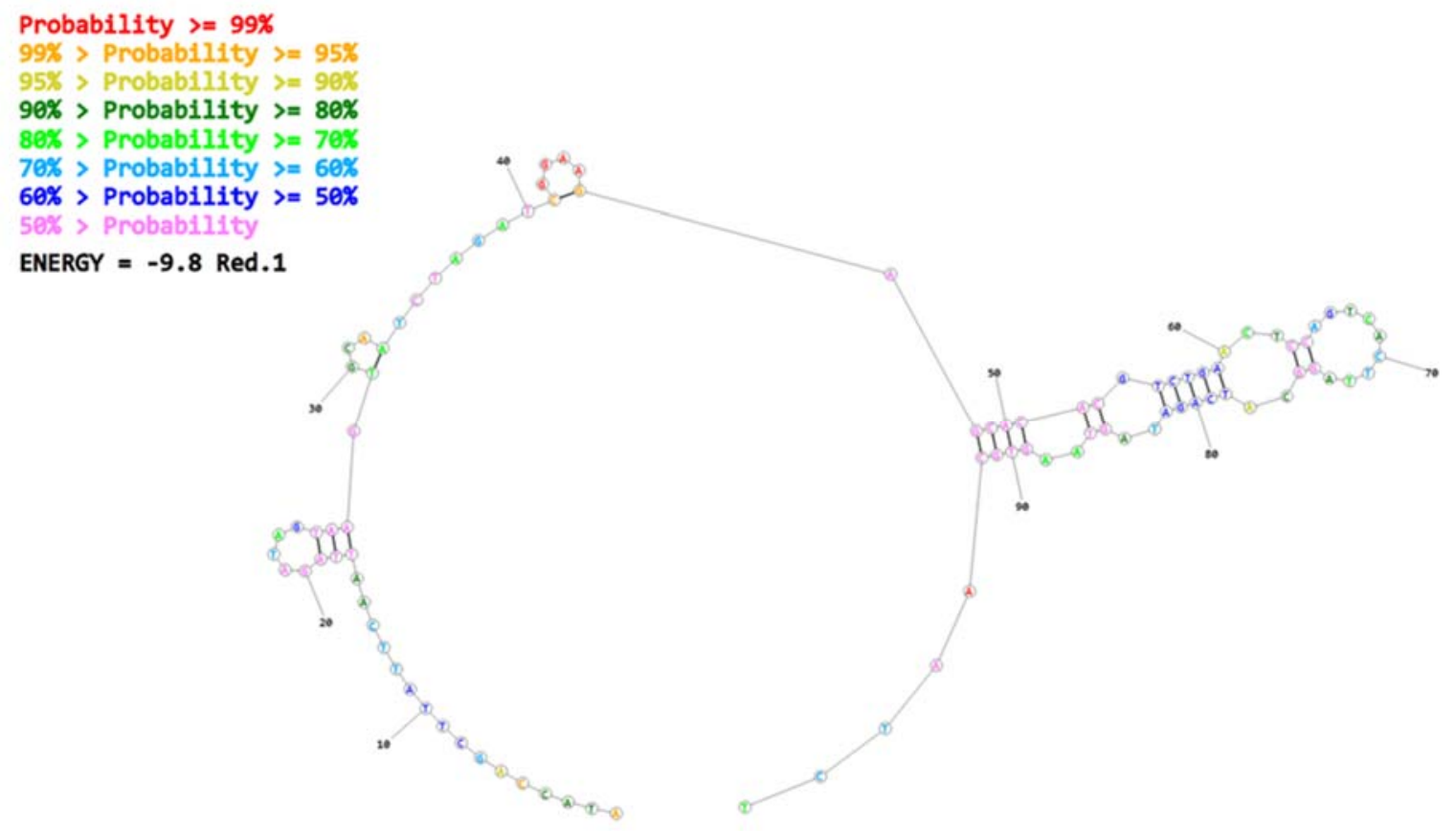

Figure 3.9: The secondary structure prediction for Red.1 generated by RNAstructure software. The colour represents the probability of each base pair as a percent.

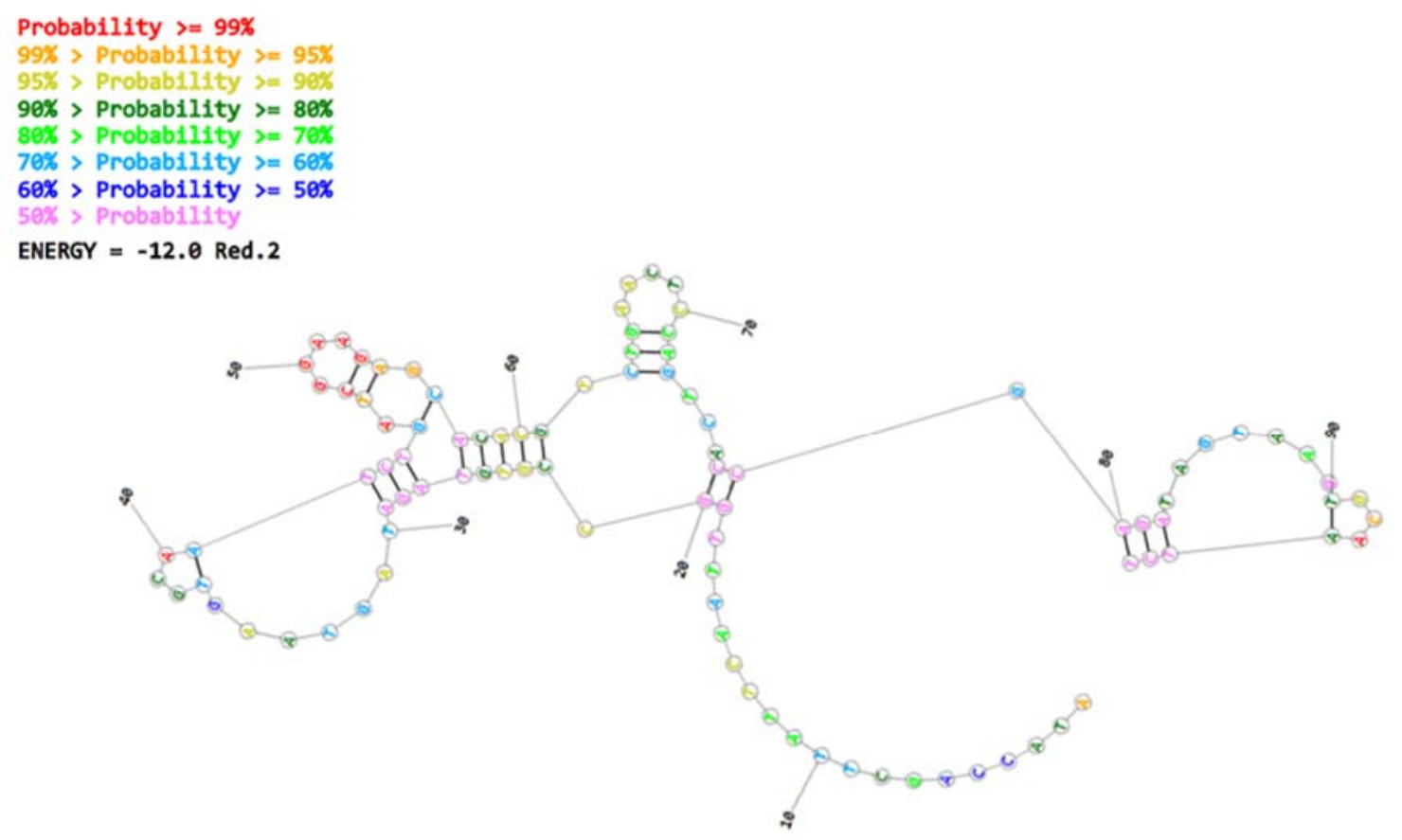

Figure 3.10: The secondary structure prediction for Red.2 generated by RNAstructure software. The colour represents the probability of each base pair as a percent. 
Each aptamer showed a variety of stem-loop structures as the predominant secondary structures. These types of stem-loop secondary structures are commonly found to be implicated in target-binding. ${ }^{26,294}$ This computational prediction of aptamer structure was a useful starting place, but did not consider more complex structure formation such as G-quadruplexes or the interaction of target molecules. The propensity for aptamer candidates to form G-quadruplex structures was examined computationally using QGRS mapper software (http://bioinformatics.ramapo.edu/QGRS/index.php), which can identify sequences that may form G-quadruplex domains. From this software, no G-quadruplex domains were found in any of the aptamer candidates. When examining the base compositions of the candidate sequences, it was observed that Ser.1, Ser.2 and Ran.2 had a slightly elevated proportion of guanine compared to the other three nucleotides, shown in Table 3.4.

Table 3.4: Distribution of nucleotides in each L-serine-binding aptamer candidate.

\begin{tabular}{|c|c|c|c|c|c|c|}
\hline Nucleotide & Ser.1 & Ser.2 & Ran.1 & Ran.2 & Red.1 & Red.2 \\
\hline A & 27.5 & 25 & 32.99 & 30.93 & 34.02 & 30.93 \\
\hline T & 16.25 & 27.5 & 26.80 & 29.90 & 25.77 & 24.74 \\
\hline G & 28.75 & 26.25 & 19.59 & 26.80 & 19.59 & 22.68 \\
\hline C & 27.5 & 21.25 & 20.62 & 12.37 & 20.62 & 21.65 \\
\hline
\end{tabular}

To verify if this elevated guanine content caused G-quadruplexes to form in these aptamer candidates, variable-temperature UV-Vis spectroscopy was used. Hypochromicity observed at $295 \mathrm{~nm}$ is indicative of a G-quartet chromophore, while hyperchromicity at $260 \mathrm{~nm}$ is indicative of duplex formation. ${ }^{295}$ All aptamer candidates were examined while undergoing thermal denaturation from $20-80^{\circ} \mathrm{C}$, and a representative melting curve is shown in Figure 3.11 for Ser. 1 at $260 \mathrm{~nm}$ and $295 \mathrm{~nm}$. 

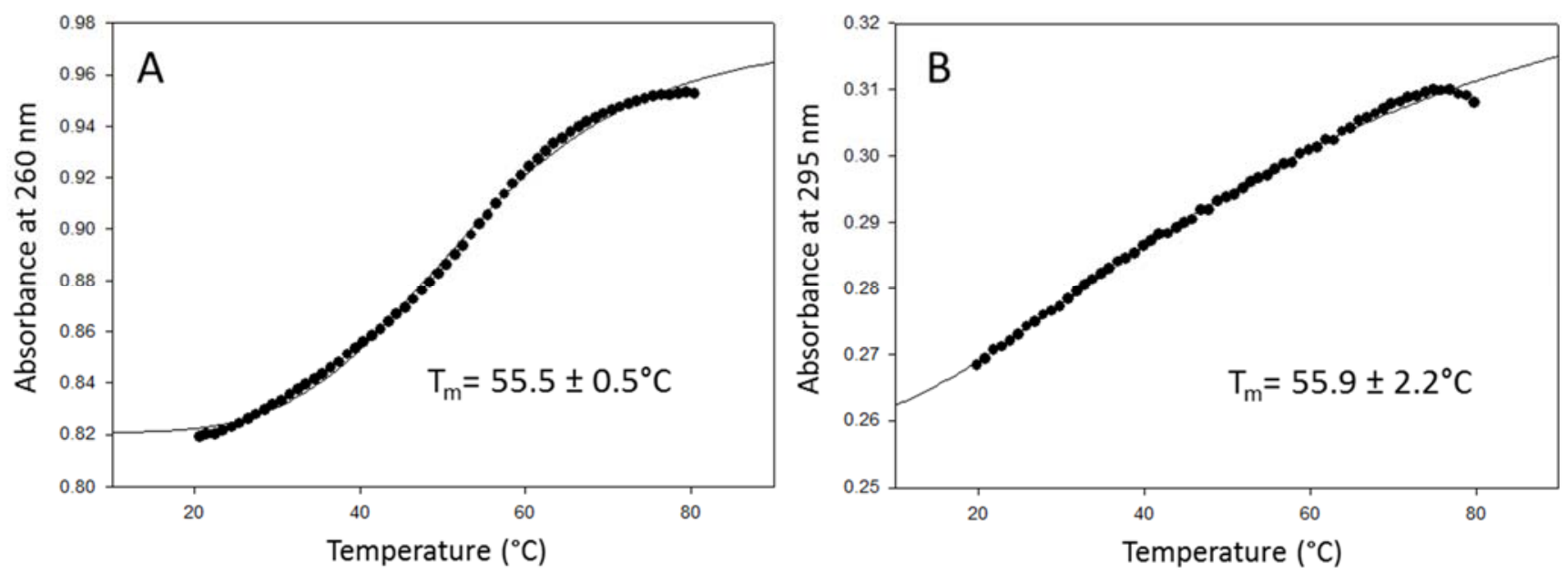

Figure 3.11: Thermal denaturation of Ser.1 from $20-80^{\circ} \mathrm{C}$ examined by $\mathrm{UV}-\mathrm{V}$ is spectroscopy. SigmaPlot software was used to fit the curves using the standard curves analysis tool, and the error shown was from the fit.

No hypochromicity was observed in any of the aptamer candidates, which agreed with the computational analysis suggesting no G-quadruplexes were formed. Hyperchromicity was observed in all aptamer candidates suggesting the melting of stem-loop structures. Each aptamer candidate was incubated with $200 \mu \mathrm{M}$ L-serine and the thermal denaturation was examined again at $260 \mathrm{~nm}$, with the resulting melting temperatures displayed in Table 3.5. 
Table 3.5: Melting temperatures obtained from variable-temperature UV-Vis analysis at $260 \mathrm{~nm}$, and examined using the standard curves analysis tool in SigmaPlot software. The error represents the standard deviation between three trials.

\begin{tabular}{ccc}
\hline Aptamer & $\mathbf{T}_{\mathbf{m}}$ at 260nm & $\mathbf{T}_{\mathbf{m}}$ with target at 260nm \\
\hline Ser.1 & $53.3 \pm 2.1$ & $48.2 \pm 4.7$ \\
\hline Ser.2 & $37.7 \pm 1.0$ & $37.6 \pm 2.2$ \\
\hline Ran.1 & $42.9 \pm 2.1$ & $49.6 \pm 3.6$ \\
Ran.2 & $40.5 \pm 2.2$ & $40.1 \pm 2.3$ \\
\hline Red.1 & $41.2 \pm 2.4$ & $41.5 \pm 4.4$ \\
\hline Red.2 & $41.7 \pm 1.6$ & $45.4 \pm 3.2$ \\
\hline
\end{tabular}

Significant changes in the melting temperatures were not observed after target incubation (confirmed using t-test), which could suggest that target-binding does not stabilize or destabilize the aptamer structures, or that L-serine is too small to affect a structural change observable by $\mathrm{UV}-\mathrm{V}$ is spectroscopy.

\subsubsection{Minimer determination}

Before moving selected aptamers into their intended applications, it can be useful to find the minimal aptamer, termed minimer. A full length aptamer contains two primer-binding regions flanking the long randomized region. Not every base in the aptamer may be involved in the target-binding interaction, and by eliminating bases not implicated in binding, the cost of the aptamer can be reduced, and in some cases the affinity can be improved. ${ }^{286,289}$ From section 3.5.3, it was determined that the Red.1 aptamer had the most affinity for L-serine, and thus was 
used for minimer determination. Section 3.5.4 gave the predicted structure of Red.1, and from this minimer sequences were designed.

A common method for obtaining a minimer is to remove the primer-binding regions used for PCR during the selections. ${ }^{280}$ The Random Region minimer was generated by removing the 18 base primer-binding regions from the 5' and 3' ends of the Red.1 aptamer. Figure $3.12 \mathrm{~A}$ shows the structural prediction for the full length Red.1 aptamer. From this structural prediction, it appears that the primer-binding regions may be involved in secondary structures that could be important for binding. Minimers were designed to encompass the two stem loops on the 5' end (5' Loop), and the larger stem loop structure on the 3' end (3' Loop), seen in Figure 3.12. Since the 3 ' primer-binding region was involved in a stem structure, but not part of the loop, a minimer was designed with this region removed (Minus 3' primer). The sequences of the four minimers designed from Red.1 are displayed in Figure 3.12 B. 


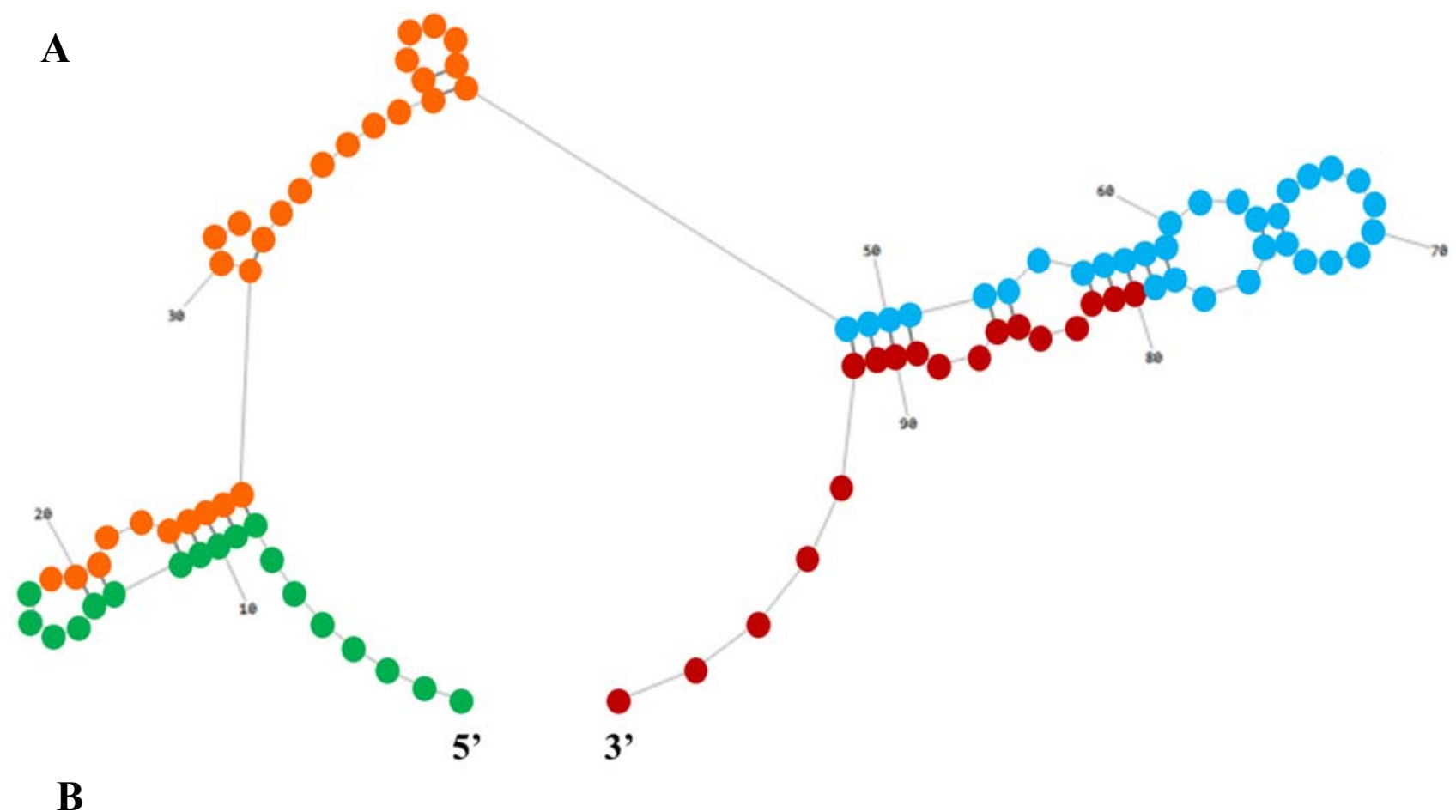

\begin{tabular}{|c|c|}
\hline Red.1 Minimer & Sequence \\
\hline 5' Loop & $\begin{array}{l}\text { ATACCAGCTTATTCAATTAGATAGTAAGTGCAATCTAGATC } \\
\text { GGAAGA }\end{array}$ \\
\hline 3'Loop & $\begin{array}{l}\text { GCACACGTCTGAACTCCAGTCACTTAGGCATCAGATAGTA } \\
\text { AGTGCAATCT }\end{array}$ \\
\hline Random Region & $\begin{array}{l}\text { AGATAGTAAGTGCAATCTAGATCGGAAGAGCACACGTCTG } \\
\text { AACTCCAGTCACTTAGGCATC }\end{array}$ \\
\hline Minus 3' Primer & $\begin{array}{l}\text { ATACCAGCTTATTCAATTAGATAGTAAGTGCAATCTAGATC } \\
\text { GGAAGAGCACACGTCTGAACTCCAGTCACTTAGGCATC }\end{array}$ \\
\hline
\end{tabular}

Figure 3.12: Minimers were designed from full length Red.1 aptamer. (A) Red.1 structure, where the colours of each segment describe the design of minimer sequences. The green and red portions of this sequence represent the 5 ' and 3' primer-binding regions, respectively. (B) Each minimer sequence designed from the Red.1 aptamer sequence, where the colours of the sequences reflect their position in the Red.1 aptamer sequence.

The apparent $\mathrm{K}_{\mathrm{d}}$ of these minimer sequences were determined by 2bind in Germany using microscale thermophoresis. The minimer sequences were ordered from IDT with 5' cy5 modifications, and mixed with varying concentrations of L-serine in solution. Figure 3.13 shows the interactions of each Red.1 minimer with L-serine in solution. The 5' Loop minimer and the Random Region minimer showed apparent $\mathrm{K}_{\mathrm{dS}}$ of $9.6 \pm 4.4 \mu \mathrm{M}$ and $5.2 \pm 2.8 \mu \mathrm{M}$, respectively. 
The 3' Loop minimer and the Minus 3' Primer minimers were unable to bind L-serine in solution. These results could indicate that the secondary structures on the 5' end of the Red.1 aptamer were directly involved in binding L-serine, as the 5'Loop minimer bound L-serine, while the 3'Loop minimer did not. Interestingly, when both primer-binding regions were removed producing the Random Region minimer, the highest binding affinity for L-serine was achieved (Figure 3.13), but if only the 3' primer-binding region was removed producing the Minus 3' Primer minimer, the affinity for L-serine was lost (Figure 3.13). The range of structures that could be adopted by the minimer sequences depended on the available nucleotides. Removing only the 3' primer could have disrupted a necessary structure that could still be accessed when both primer-binding regions were removed. 

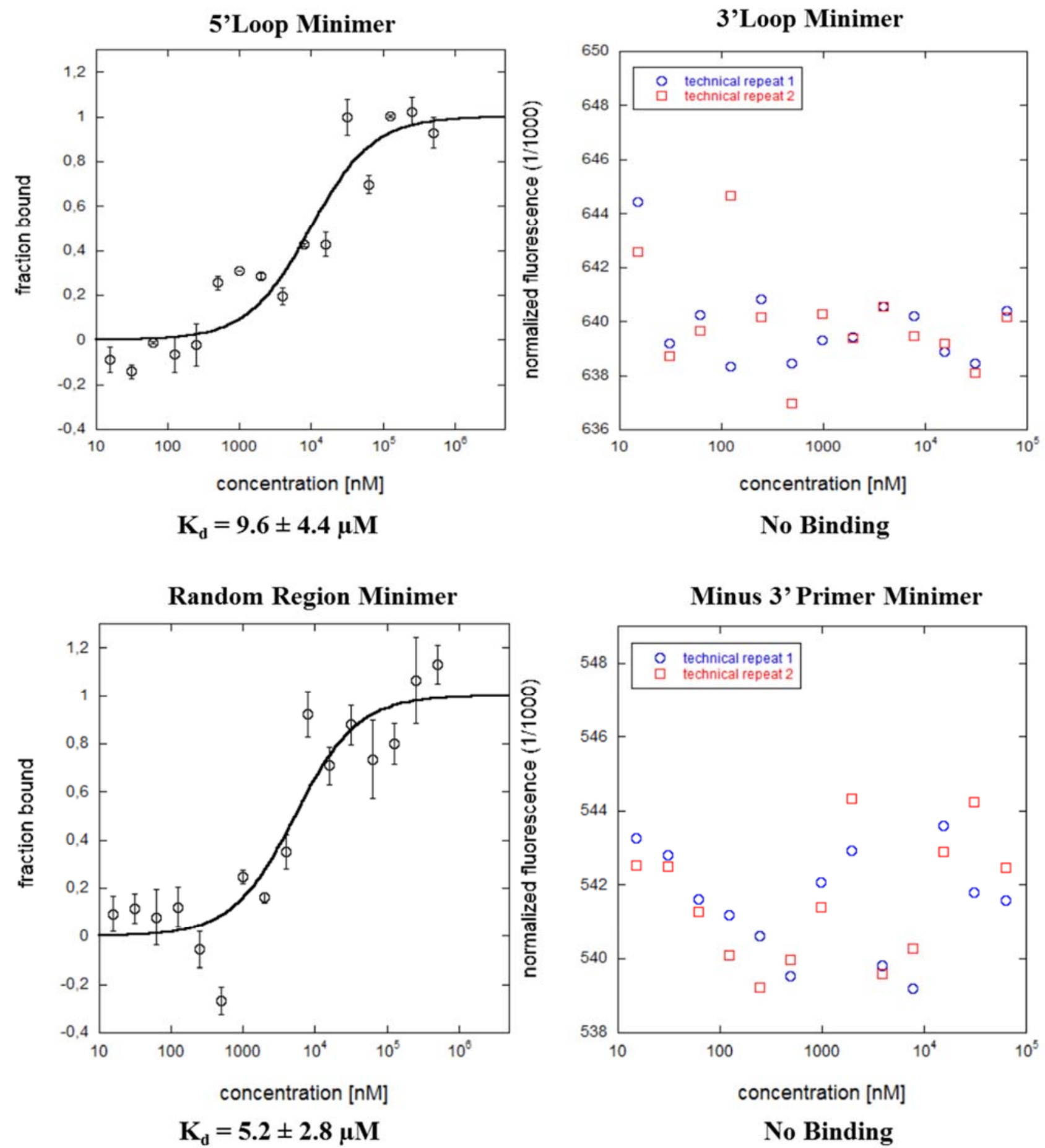

No Binding

Figure 3.13: Microscale thermophoresis examination of Red.1 minimers binding L-serine in solution. All minimers ( $5 \mathrm{nM})$ were 5 ' $\mathrm{Cy} 5$ labeled and incubated with varying concentrations of L-serine in PBS $(15 \mathrm{nM}-500 \mu \mathrm{M})$ to generate these curves, provided by 2bind, Germany. 
From MST analysis, the Random Region minimer had the highest affinity for L-serine, with the full length Red.1 aptamer following closely behind, with apparent $\mathrm{K}_{\mathrm{dS}}$ of $5.2 \pm 2.8 \mu \mathrm{M}$ and 7.9 $\pm 3.6 \mu \mathrm{M}$, respectively. As both these sequences bound L-serine with the highest affinities, RNAstructure software was used to examine the sequences for common secondary structures that could be present, and important for the L-serine-binding interaction. Figure 3.14 shows that two stem-loop structures were predicted to be shared by Red.1 and Random Region Minimer, and could be important in binding L-serine. The two 18 base primer-binding regions on the 5' and 3' ends of the Red.1 aptamer were not part of these stem-loops, and when they were removed, the affinity for L-serine was improved.
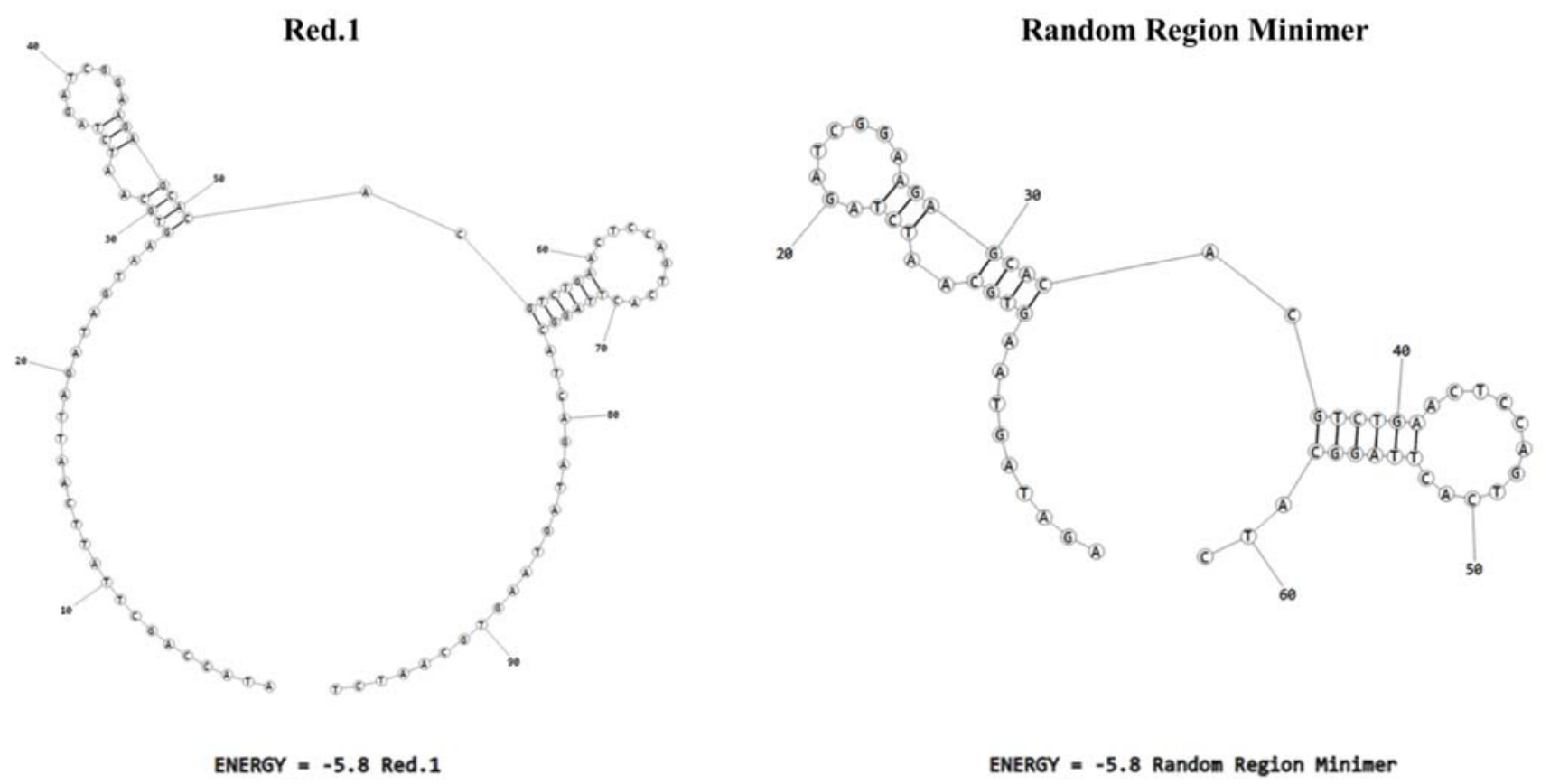

Figure 3.14: A prediction of common secondary structures shared by the full length Red.1 aptamer and its random region minimer, generated by RNAstructure software. 


\section{Examination of aptamer structures for conserved sequence regions}

In section 2.5.7, sequence homology was examined, and some conserved motifs were identified. The reverse primer-binding region had been inserted into several candidates, the 13base sequence 5'-GGAAGAGCACACG-3' was found in Ser.1, Ran.1, Red.1, and Red.2, and the short motif GATC was found in all aptamer candidates. The predicted structures that were generated for the selected aptamer candidates showed stem-loops and bulges as the predominant secondary structures (Figure 3.5 to Figure 3.10). Many aptamers have been found to interact with their targets through these loops and bulges, such as aptamers for dopamine, protein A, and ATP for example. ${ }^{294,296,297}$ The structures of the best aptamers that bound immobilized and free Lserine, Ser.1, Ran.2, and Red.1 were examined for the presence of these three conserved regions, to elucidate possible sites for L-serine-binding, shown in Figure 3.15. 

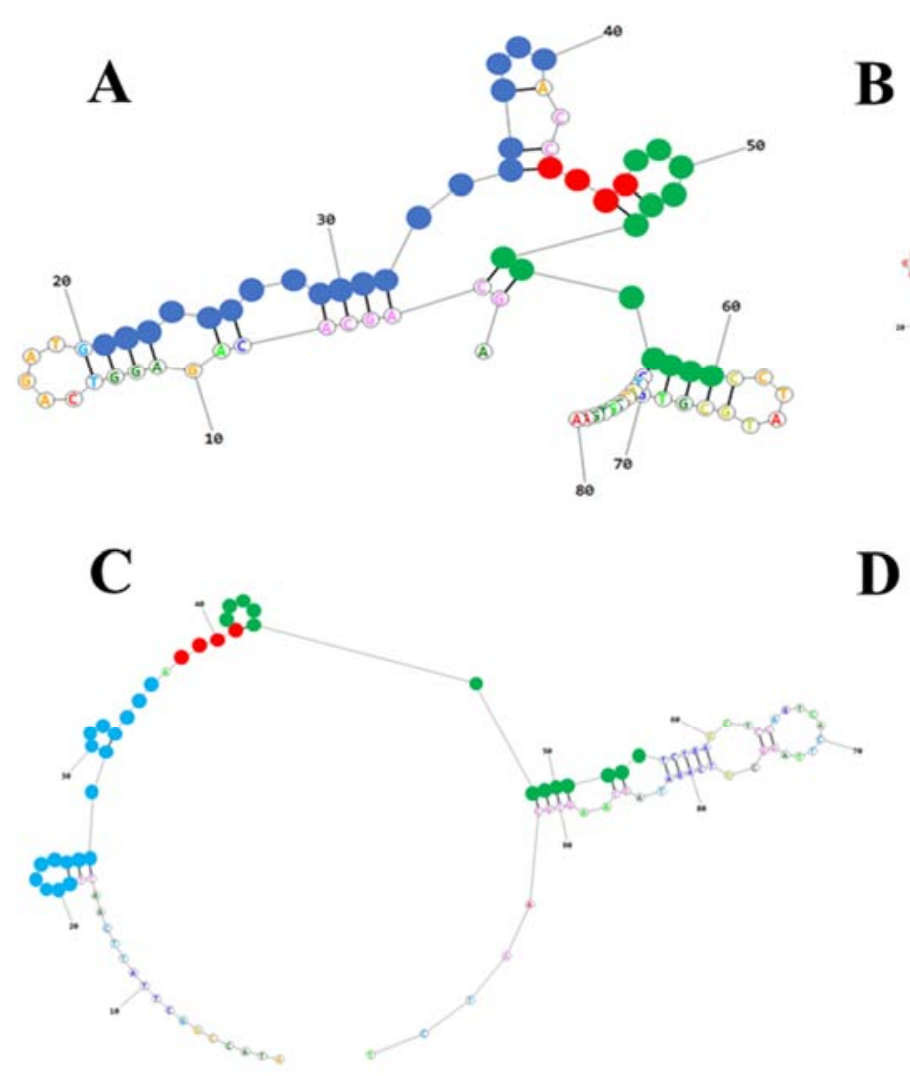

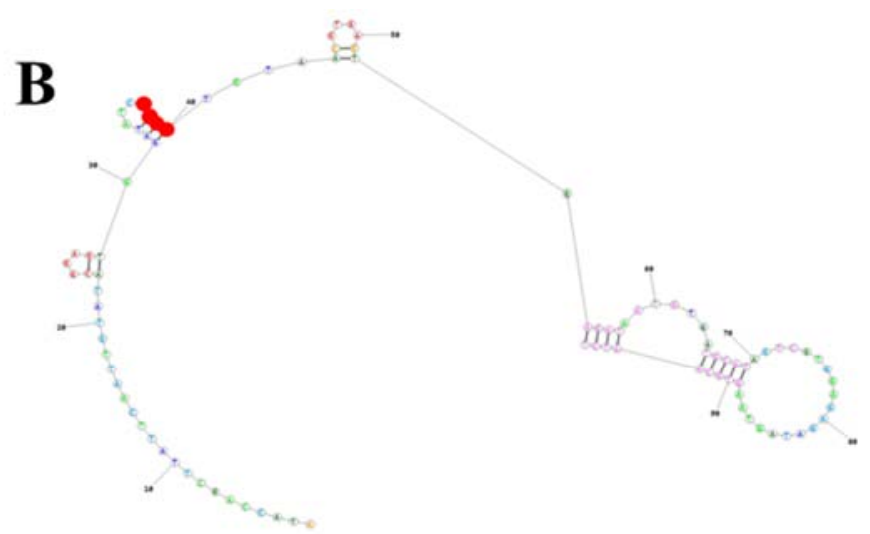

D

- GATC

- GGAAGAGCACACG

Reverse primer-binding region

CCTATGCGTGCTACCGTGAA

AGATAGTAAGTGCAATCT

Figure 3.15: Conserved sequence regions present in L-serine-binding aptamers (A) Ser.1, (B) Ran.2, and (C) Red.1. Conserved sequence motifs are indicated by a coloured circle (D).

Ser.1 contained a repeat of its reverse primer-binding region, the 13-base conserved region, as well as the GATC motif. The inserted primer-binding region was shown to participate in two bulges in a large stem-loop structure, as well as the loop portion of a second stem-loop. The 13-base conserved region also participated in the loop structure of a stem-loop. While these two conserved motifs could be regions for target interaction, the GATC conserved motif was found between two stem-loops, which would be blocked for target interaction. The Ran.2 aptamer did not contain an inserted primer-binding sequence, nor did it contain the 13-base conserved sequence. The only conserved motif found in Ran.2 was the GATC motif, which was found on the outside of a stem-loop, which could have been exposed to and interacted with Lserine in solution. 
The aptamer with the highest affinity for L-serine was Red.1, which also contained all three conserved sequence regions in areas that could have been exposed to L-serine in solution. The inserted primer-binding region was shown to participate in two loops, the GATC motif was involved in one loop, and the 13-base conserved motif was involved in a loop as well as two bulges within the stem of a stem-loop structure, shown in Figure 3.15. The Red.1 minimer also bound L-serine with high affinity, and the secondary structures that were predicted to be shared between Red.1 and its random region minimer were also examined. The conserved regions are shown in Figure 3.16.

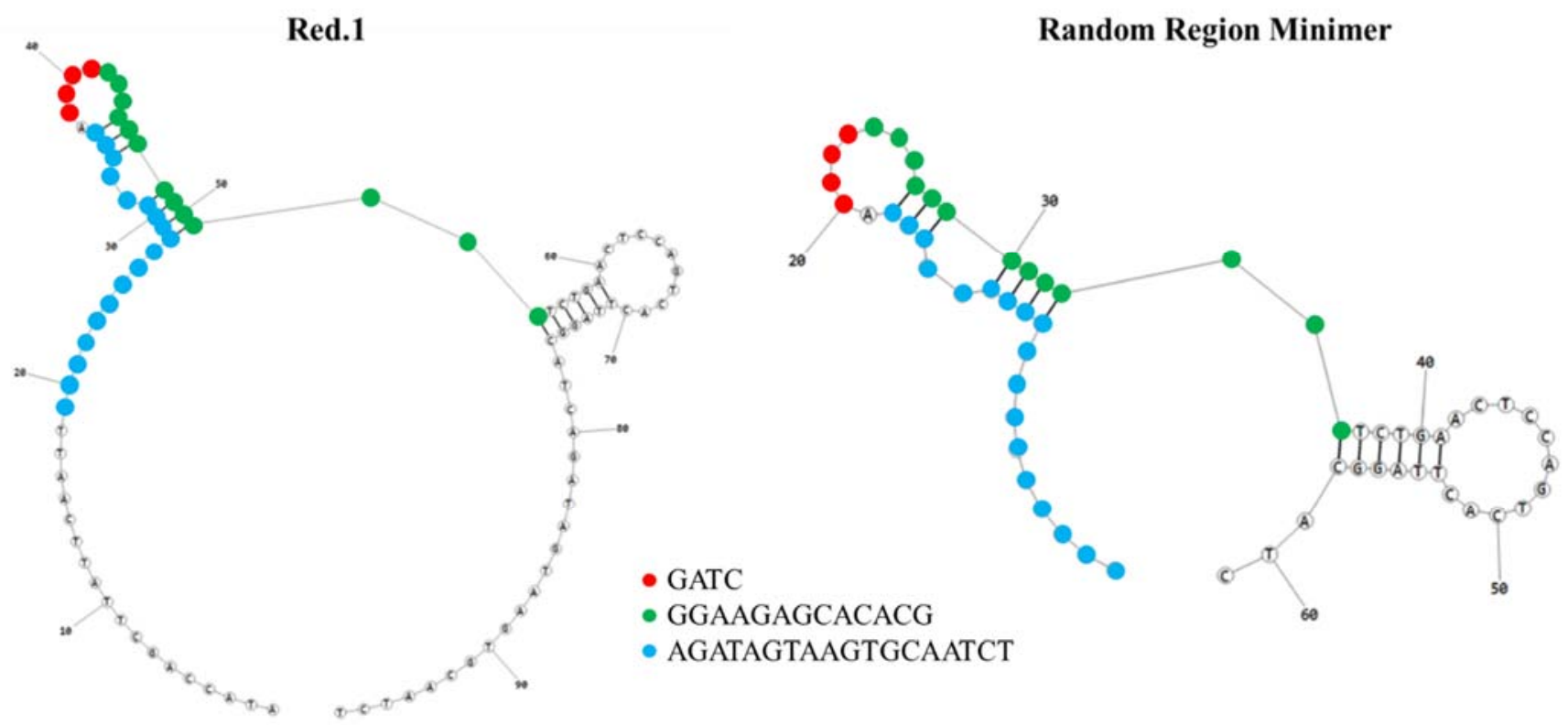

Figure 3.16: Conserved sequence motifs found in the shared predicted secondary structures of Red.1 and its random region minimer. Structure prediction was done using RNAstructure software, and conserved sequence regions are indicated by coloured circles.

The conserved sequences in Red.1 remain available to interact with the target in solution in the conformation shown in Figure 3.16. The random region minimer sequence also contained all three conserved regions. Interestingly, these predicted structures included all three conserved motifs in one stem-loop secondary structure, which could suggest this stem-loops' involvement 
in L-serine-binding. This stem-loop structure could be further studied using mutational analysis to determine its involvement in L-serine-binding. It could be possible to shorten the minimer even further by eliminating non-conserved bases on the 3 'end.

\subsubsection{Colorimetric AuNP test for aptamer and minimer L-serine-binding}

The development of colorimetric sensors for aptamer-target binding would allow for rapid results, without the need for expensive equipment or highly-trained personnel. Gold nanoparticles (AuNPs) have been used as reporter probes in aptamer-based colorimetric sensors. ${ }^{298,299}$ A dispersed solution of AuNPs appears wine red in colour, while an aggregated AuNP solution appears blue, making them a useful colorimetric tool. The introduction of salt causes the AuNPs to aggregate, turning the red solution to blue. Single-stranded DNA strands, such as aptamers, are able to adsorb onto the surface of the AuNPs, protecting them from saltinduced aggregation. ${ }^{298}$ The protection of AuNPs by ssDNA can be exploited to generate an aptamer-based AuNP sensor, demonstrated in Figure 3.17. 


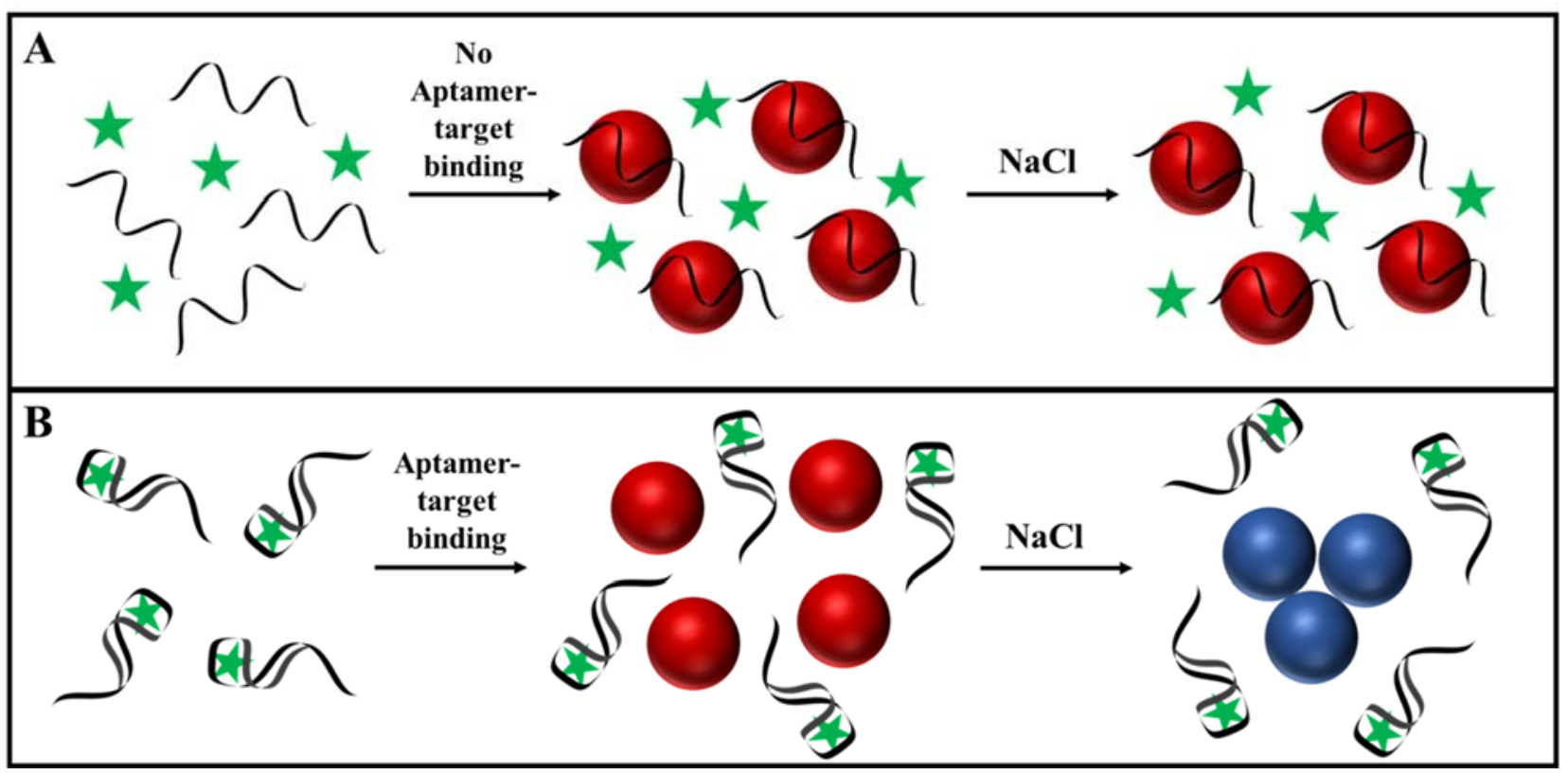

Figure 3.17: Design of an aptamer-based AuNP sensor. A) When an aptamer (black) does not bind a target (green), the aptamer is available to adsorb onto the AuNP surface (red), protecting them from salt-induced aggregation. B) When an aptamer binds a target, it no longer adsorbs to the AuNP surface, leaving the AuNPs susceptible to salt-induced aggregation (blue).

If an aptamer does not bind a target molecule, the aptamer can adsorb onto the surface of the AuNPs. When salt is introduced, the ssDNA aptamers protect the AuNPs from aggregation. If an aptamer binds a target molecule, this interaction prevents the aptamer from adsorbing onto the AuNP surface. When salt is added, these unprotected AuNPs are susceptible to salt-induced aggregation, causing a visible colour change from red to blue. McKeague et al. determined the binding affinites of multiple ochratoxin A-binding aptamers using a variety of $\mathrm{K}_{\mathrm{d}}$ tests, as well as their ability to be used in this qualitative aptamer-based gold nanoparticle colorimetric test. ${ }^{281}$ While all the aptamers examined demonstrated target-binding in multiple $\mathrm{K}_{\mathrm{d}}$ tests, only about half the studied aptamers were able to induce a colour change in the AuNP test. This varied result could be dependent on individual aptamer structures, or the dependence on a solid support for target-binding. As the Red.1 L-serine-binding aptamer was shown to bind L-serine in solution (section 3.5.2), it was examined for use in this AuNP test. 
To optimize AuNP test conditions, the amount of Red.1 needed to protect the AuNP from salt-induced aggregation was examined. It was found that $3 \mu \mathrm{L}$ of a $10 \mu \mathrm{M}$ Red.1 solution was able to protect the AuNPs from aggregation when $0.75 \mathrm{M} \mathrm{NaCl}$ was added, seen in Figure 3.18 A. L-serine was added to a final concentration of $53 \mathrm{mM}$, and aggregation with $0.75 \mathrm{M} \mathrm{NaCl}$ was again examined. The results of this AuNP test are shown in Figure 3.18 B.

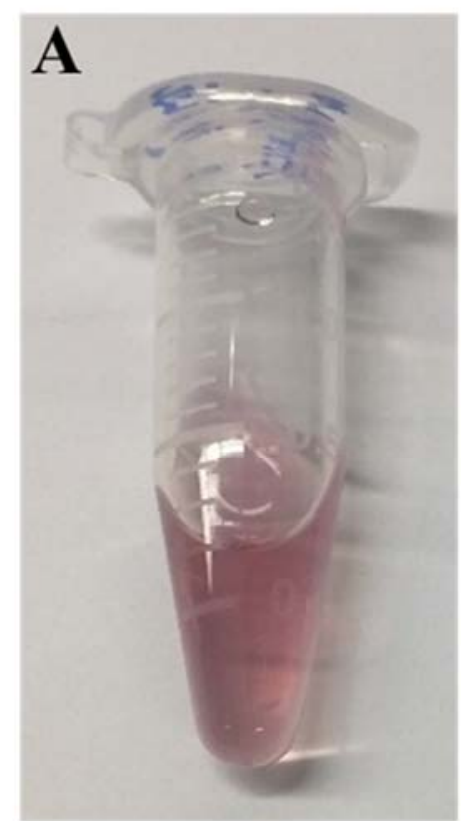

Red.1

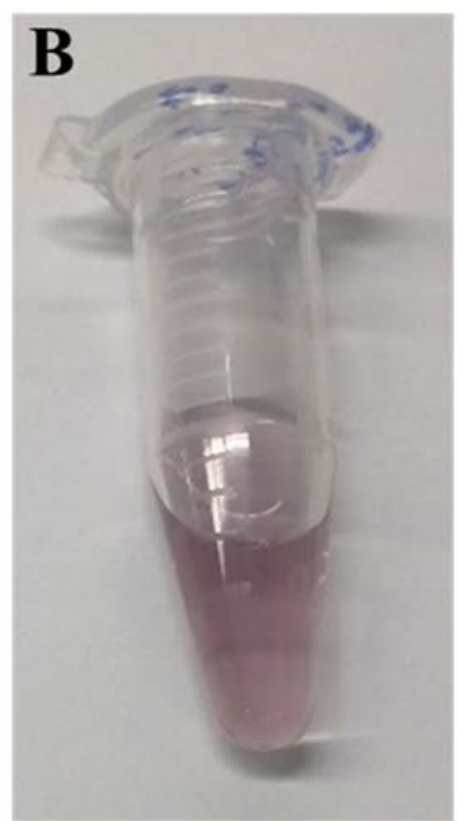

Red.1 + L-serine

Figure 3.18: L-serine binding test using AuNPs with Red.1 aptamer. A) Red.1 protects AuNPs from aggregation in the presence of $0.75 \mathrm{M} \mathrm{NaCl}$. B) In the presence of $53 \mathrm{mM} \mathrm{L}$ serine, Red.1 no longer protects AuNPs, resulting in a blue colour change.

With the addition of L-serine, a slight colour change from red to blue was observed, seen in Figure 3.18 B. This colour change was indicative of the Red.1 aptamer binding L-serine in solution, leaving the AuNPs susceptible to salt-induced aggregation. The colour change observed was not dramatic, and required a high concentration of L-serine to be added. The Red.1 aptamer was very long at 97 bases, and since L-serine was a small molecule target, it was possible that 
the aptamer was able to bind L-serine while also interacting with the AuNP surface, suppressing the aggregation. This was observed by Tian et al. using the acetamiprid aptamer in the same AuNP test. ${ }^{300}$ By truncating non-essential nucleotides from the full length aptamer, they were able to improve the colorimetric sensitivity of the test by 3.3 fold. The Random Region Minimer was generated in section 3.5.5 by truncating the primer-binding regions of the Red.1 aptamer, shortening it from 97 bases to 61 bases. This minimer was examined for use in this colorimetric test.

Using the Random Region Minimer, a lower salt concentration was required to cause aggregation, as $3 \mu \mathrm{L}$ were able to protect the AuNPs from the addition of $0.5 \mathrm{M} \mathrm{NaCl}$, but not $0.75 \mathrm{M} \mathrm{NaCl}$. This was likely due to the truncated sequence being less able to cover the AuNP surface compared to the full length aptamer. L-serine was added at a final concentration of 546 $\mu \mathrm{M}$, and left to incubate until aggregation could be observed, shown in Figure 3.19. 


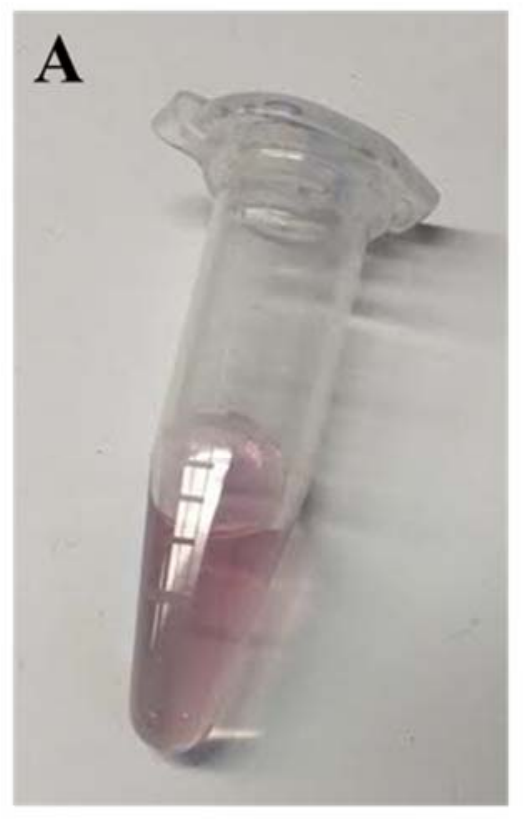

RR_Minimer

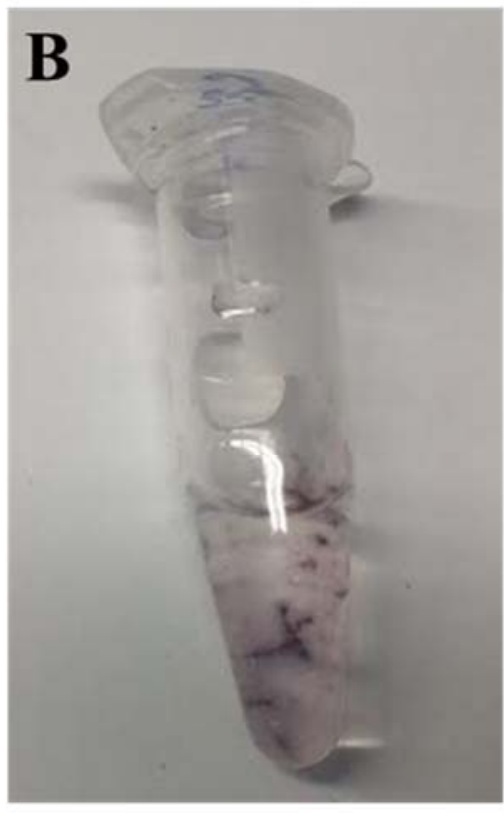

RR_Minimer + L-serine

Figure 3.19: L-serine binding test using AuNPs with Random Region Minimer. A) Red.1 protects AuNPs from aggregation in the presence of $0.5 \mathrm{M} \mathrm{NaCl}$. B) In the presence of 546 $\mu M$ L-serine, Red.1 no longer protects AuNPs, resulting in AuNP aggregation after one week incubation.

Using the Random Region Minimer, a more obvious change was observed with a lower target concentration, with the AuNPs aggregating in the presence of $546 \mu \mathrm{M} \mathrm{L}$-serine. Since non-essential nucleotides had been truncated, it was possible that fewer excess bases remained available to interact with the AuNPs when the minimer was bound to L-serine. To lower the time required for a visible colour change, and to increase sensitivity, the test conditions would need to be optimized. Many factors affecting the adsorption of ssDNA onto AuNPs, including ssDNA length and salt concentration could be examined to determine optimal test conditions. ${ }^{301}$ The visible colour change observed in the presence of the target L-serine suggested that an aptamerbased colorimetric AuNP sensor could be created to detect L-serine. A simple colorimetric test to 
detect the presence of L-serine in soil could allow farmers to identify periods of increased nitrogen uptake to better inform fertilizer application.

\subsubsection{Preparation and assessment of an L-serine-binding Spiegelmer}

Transitioning aptamers into sensor and therapeutic applications often requires some sort of modification for aptamers to improve biological and chemical stability. Spiegelmers are mirror-image aptamers, containing L-deoxyribonucleotides while traditional DNA aptamers are composed of D-deoxyribonucleotides. Using L-deoxyribonucleotides affords Spiegelmers a resistance to nucleases that is orders of magnitude higher than traditional aptamers, as nucleases are stereoselective. ${ }^{3}$ Serine contains a chiral carbon, generating L/D enantiomers. The selection experiments were completed using L-serine as the target, with no counter selection against its enantiomer D-serine. Synthesizing the Random Region Minimer as a Spiegelmer allowed us to investigate the stereoselectivity of the binding pocket.

The Random Region Minimer was synthesized using L-phosphoramidites to generate its corresponding Spiegelmer. This Spiegelmer and the Random Region Minimer were labeled with 5' Cy5 and incubated with a range of L-serine and D-serine concentrations to determine apparent $\mathrm{K}_{\mathrm{d}} \mathrm{s}$ by microscale thermophoresis. The resulting binding curves were obtained from $2 \mathrm{bind}$, and are displayed in Figure 3.20, and the apparent $\mathrm{K}_{\mathrm{d}}$ for each interaction are displayed in Table 3.6. The selectivity of the minimer and Spiegelmer were also investigated by examining their interactions with Tris and L-glycine by MST. These results are also displayed in Table 3.6. 

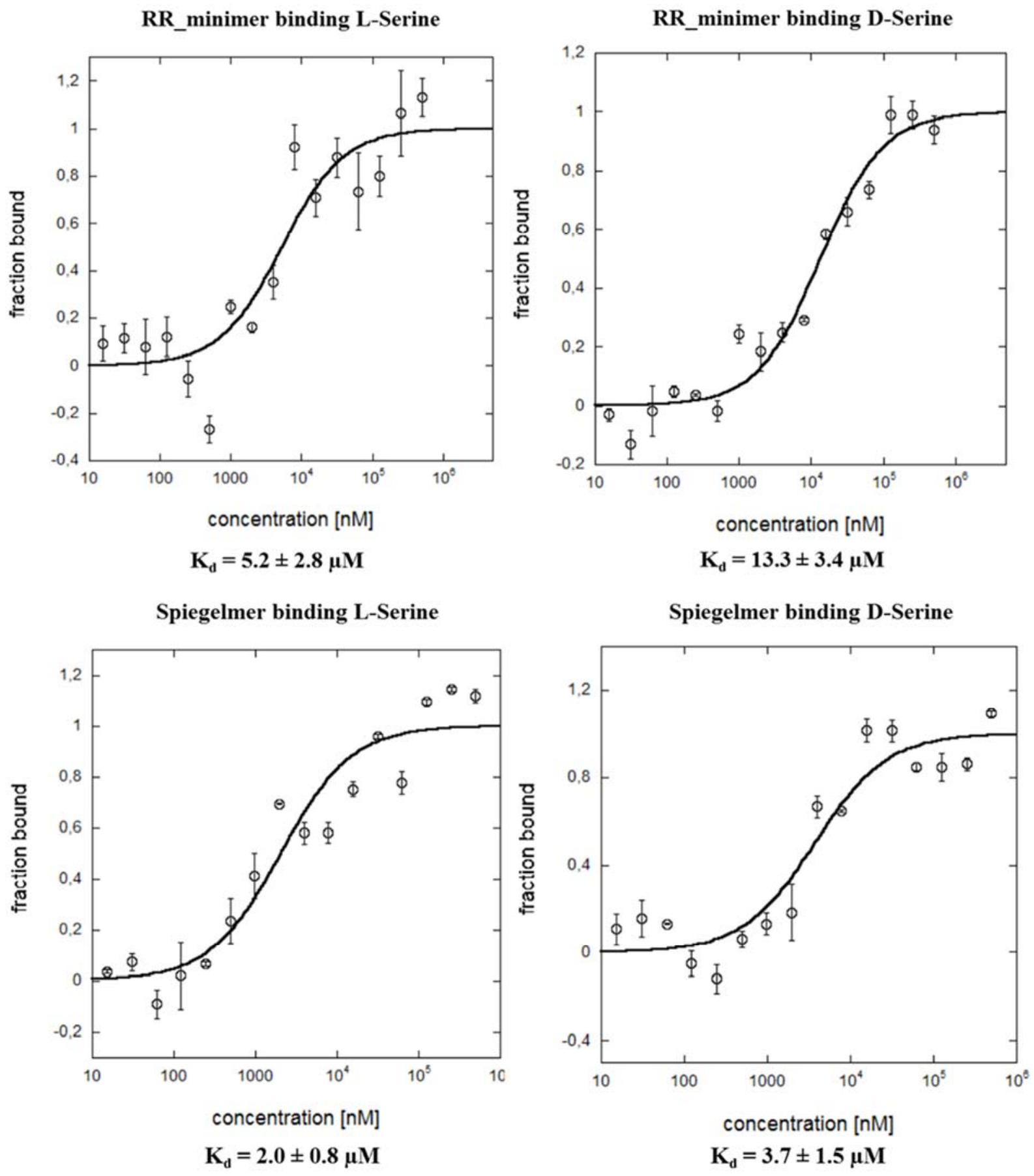

Figure 3.20: Microscale thermophoresis analysis of Random Region Minimer and its corresponding Spiegelmer binding $L$ and $D$-serine in solution. The minimer and Spiegelmer (20 nM) were 5' $\mathrm{Cy} 5$ labeled and monitored after incubation with varying concentrations of $L$ - and D-serine in PBS $(15 \mathrm{nM}-500 \mu \mathrm{M})$. The results were provided by 2bind, Germany. 
Table 3.6: Apparent $K_{d}$ s for the RR_Minimer and Spiegelmer binding L- and D-serine, Lglycine, and Tris obtained by MST.

\begin{tabular}{|c|c|c|c|c|}
\hline \multirow{2}{*}{ Minimer } & \multicolumn{3}{|c|}{ Apparent $\mathbf{K}_{\mathbf{d}}(\boldsymbol{\mu M})$} \\
\hline & L-Serine & D-Serine & L-Glycine & Tris \\
\hline RR_Minimer & $5.2 \pm 2.7$ & $13.3 \pm 3.4$ & $19.8 \pm 4.9$ & $0.2 \pm 0.06$ \\
\hline Spiegelmer & $2.0 \pm 0.8$ & $3.7 \pm 1.5$ & $13.7 \pm 8.6$ & $5.2 \pm 1.9$ \\
\hline
\end{tabular}

The Random Region minimer bound the target L-serine with an apparent $\mathrm{K}_{\mathrm{d}}$ of $5.2 \pm 2.7$ $\mu \mathrm{M}$ and bound D-serine more weakly with an apparent $\mathrm{K}_{\mathrm{d}}$ of $13.3 \pm 3.4 \mu \mathrm{M}$. The Spiegelmer bound the target L-serine most strongly, with an apparent $\mathrm{K}_{\mathrm{d}}$ of $2.0 \pm 0.8 \mu \mathrm{M}$, while binding Dserine only slightly more weakly with an apparent $\mathrm{K}_{\mathrm{d}}$ of $3.7 \pm 1.5 \mu \mathrm{M}$. Since the Spiegelmer was able to bind strongly to both serine enantiomers, it is likely that serine's chiral carbon was not part of the binding site, or that the binding pocket was loose enough to allow either serine enantiomer to bind. During the selections, serine's amino group was used to immobilize L-serine to the sepharose solid support and was therefore unlikely involved in the binding interaction. The remaining functional group was serine's alkyl hydroxyl side-chain which was most likely the binding site for the Random Region Minimer and Spiegelmer. The minimer and Spiegelmer had less affinity for L-glycine, with apparent $\mathrm{K}_{\mathrm{d}} \mathrm{S}$ of $19.8 \pm 4.9 \mu \mathrm{M}$, and $13.7 \pm 8.6 \mu \mathrm{M}$, respectively which could be attributed to L-glycine's lack of hydroxyl group. Both the minimer and Spiegelmer were able to tightly bind Tris, with apparent $\mathrm{K}_{\mathrm{d}} \mathrm{S}$ of $0.23 \pm 0.065 \mu \mathrm{M}$ and $5.2 \pm 1.9$ $\mu \mathrm{M}$, respectively. Tris offers three alkyl hydroxyl groups as binding sites, which could explain the affinity the minimer and Spiegelmer showed. Of all the aptamer candidates and minimers tested, the Spiegelmer showed the highest affinity for the target L-serine, with an apparent $\mathrm{K}_{\mathrm{d}}$ of 
$2.0 \pm 0.8 \mu \mathrm{M}$, while it could also provide the nuclease resistance needed to bridge aptamers into useful sensors and smart materials.

\subsubsection{Examining the nuclease resistance of an L-serine-binding Spiegelmer}

Incorporating aptamers into useful applications is often challenged by their rapid nuclease degradation. As they are biopolymers, naturally occurring nuclease enzymes can quickly degrade them. Aptamers circulating in the bloodstream have shown to be degraded within minutes, for example. ${ }^{302}$ This circulation time has been extended by using modified bases and PEG conjugation, however such modifications increase the cost, and could interfere with target-binding. Nucleases had to be considered when designing an aptamer for use in a smart fertilizer as well, as extracellular nucleases get released by soil bacteria and fungi. ${ }^{303}$ The Lserine binding Spiegelmer could provide nuclease resistance without negatively affecting targetbinding.

To examine the stability of the L-serine-binding Spiegelmer compared to the Random Region Minimer, each one was exposed to the nuclease DNase I at $37^{\circ} \mathrm{C}$ for a range of time points. DNase I is an endonuclease which non-specifically cleaves DNA, resulting in smaller oligonucleotide fragments. The results of this nuclease incubation with the Random Region Minimer and the corresponding Spiegelmer are displayed in Figure 3.21. 
Minimer

Spiegelmer

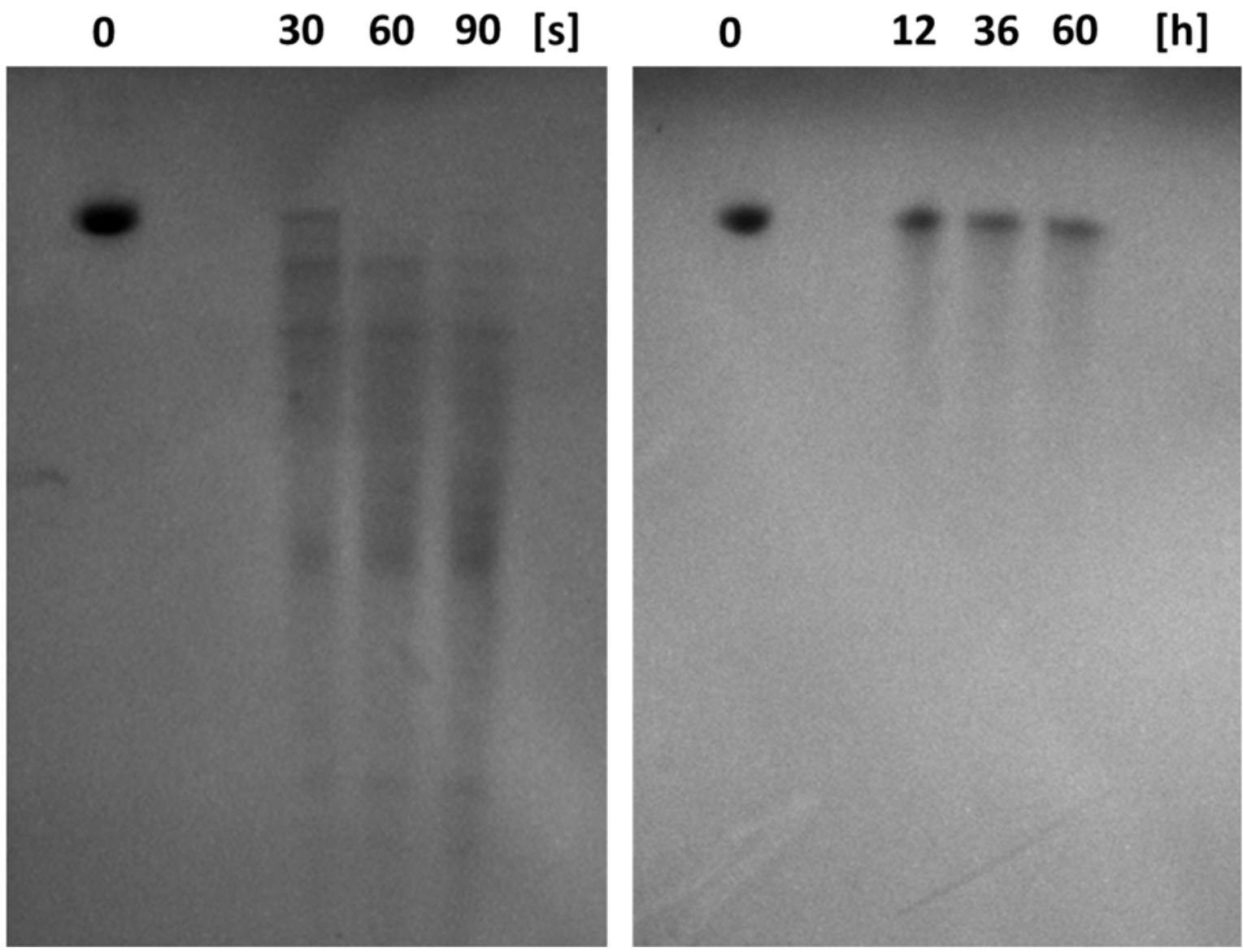

Figure 3.21: The stability of the L-serine-binding minimer (D-DNA) and Spiegelmer (LDNA) in the presence of DNase I at $37^{\circ} \mathrm{C}$. After the time period indicated (time scale for the minimer is in seconds, while the time scale for the Spiegelmer is in hours), EDTA was added, DNA was heated to $90^{\circ} \mathrm{C}$ to inactivate the enzyme, and was stored at $-20^{\circ} \mathrm{C}$ until $19 \%$ denaturing PAGE could be performed.

For the Random Region Minimer, which was composed of the D-DNA enantiomer found in nature, nuclease degradation proceeded quickly. After 30 seconds of DNase I exposure, the full length minimer could still be observed, but several degradation products were also visible. After only one minute of DNase I exposure, no full length minimer could be observed. The Spiegelmer, composed of L-DNA, was exposed to DNase I at $37^{\circ} \mathrm{C}$ for 60 hours, and even after this extended exposure, full length Spiegelmer was observed. The fainter bands observed over 
time suggested a low level of Spiegelmer degradation, however the full length Spiegelmer was still predominant. Naturally occurring nucleases show stereospecificity toward the degradation of D-DNA, allowing the L-DNA of Spiegelmers to escape degradation. ${ }^{304}$ Using the L-serine binding Spiegelmer for further applications would maintain the binding affinity while also providing the nuclease resistance needed for incorporation into smart fertilizer applications.

\subsection{Conclusions}

Binding affinity experiments showed that Ser.2, Ran.1, and Red.2 bound L-serine only when it was immobilized, while Ser.1, Ran.2, and Red.1 all bound immobilized and free Lserine. Each SELEX experiment generated an L-serine-binding aptamer, suggesting the use of an original selection library, randomized aptamer library, and a library redirected from another selection were all valid SELEX strategies. Red.1 aptamer had the highest affinity for L-serine in solution with an apparent $\mathrm{K}_{\mathrm{d}}$ of $7.9 \pm 3.6 \mu \mathrm{M}$. Removing the primer-binding regions from Red.1 improved the binding affinity to $5.2 \pm 2.7 \mu \mathrm{M}$, and one stem-loop structure containing three conserved sequence motifs was predicted to be important for L-serine-binding. A serine-binding Spiegelmer was generated which suggested serine's alkyl hydroxyl side-chain was important for aptamer binding. The Spiegelmer had the highest affinity for L-serine with an apparent $\mathrm{K}_{\mathrm{d}}$ of 2.0 $\pm 0.8 \mu \mathrm{M}$. This Spiegelmer was also shown to resist nuclease degradation for at least 60 hours.

This Spiegelmer has the affinity for L-serine and stability from nucleases which could allow it to be incorporated into aptamer-smart materials for fertilizer applications. 
4 Synthesis and characterization of aptamer-polymersomes 


\subsection{Chapter objectives}

This chapter describes the synthesis of amphiphilic aptamer-lipid conjugates and their incorporation into self-assembled polymersomes to be investigated as potential targeted-delivery vehicles. The morphology of aptamer-polymersomes was examined using TEM. The effects of the hydrophobic chain-length on polymersome morphology and stability were investigated. Finally, target-binding ability of the aptamer-lipid conjugates and aptamer-polymersomes was investigated using fluorescence enhancement, equilibrium dialysis and fluorescence anisotropy.

\subsection{Statement of contributions}

The aptamer-conjugate polymersome system was conceptualized by Y. Sultan and M.C. DeRosa. Aptamer conjugates and aptamer-liposomes were synthesized by E. Mastronardi for extrusion and target-binding studies, and by E. Mastronardi and I. Povel for stability studies. Fluorescence anisotropy measurements were performed by E. Mastronardi with help from E.M. McConnell. Samples for microscale thermophoresis were prepared by E. Mastronardi, and run by 2 bind in Germany. TEM imaging was performed by J. Wang at the Nano Imaging Facility at Carleton University. All image analysis was done by E. Mastronardi. 


\subsection{Introduction}

\subsubsection{Targeted-delivery using aptamers}

Aptamers can be used in targeted-delivery applications in many fields, but have predominantly been investigated for medical uses. Aptamers have been employed in targeted delivery strategies, bringing therapeutic compounds directly to specific pathogens or diseased tissue, for example. Aptamers have been used to improve the specificity of chemotherapeutics, toxins, and nanoparticles. Nanoparticles and liposomes can encapsulate therapeutic compounds, and can be directed to target tissues using aptamers. ${ }^{305-308}$ For example, an RNA aptamer specific for the prostate-specific membrane antigen (PSMA) was conjugated to the surface of polymer nanoparticles encapsulating the drug docetaxel, and the uptake of these targeted nanoparticles by prostate cancer cells was examined. ${ }^{305}$ These aptamer-targeted nanoparticles resulted in significantly higher cellular toxicity compared to non-targeted nanoparticles, suggesting the aptamer's recognition of the surface antigen facilitated uptake of these drug-loaded nanoparticles. These aptamer-targeted nanoparticles showed reduced toxicity to mice, and increased efficacy, as mice receiving the targeted nanoparticles showed a $100 \%$ survival rate compared to only $14 \%$ for mice receiving docetaxel only.

The Tan group used this aptamer-targeting strategy to develop the first aptamerconjugated multifunctional liposome nanostructure to be used for targeted delivery. ${ }^{308}$ To the liposome surface, the authors conjugated the sgc 8 aptamer, which had specificity for leukemia CEM-CCRF cells. The aptamer-conjugated liposomes were able to selectively bind the target leukemia cells, with no binding observed for non-target cells. In addition to binding, the aptamer-liposomes facilitated the delivery of a fluorophore cargo molecule that had been encapsulated within the liposome. Liposomes without the sgc8 aptamer were unable to deliver 
the fluorophore to the cells. The authors suggested that by using aptamers targeting a variety of cells, this aptamer-conjugated liposome system could be generalized to any target. These examples show how aptamers can successfully be used to bring cargo to specific tissues but this strategy could be generalized to a variety of targets. Incorporating aptamers within nanostructures could allow responsive materials to be designed.

\subsubsection{Responsive DNA-nanostructures}

The encapsulation of cargo molecules to improve their stability and prolong their release has been a strategy employed in medicine and agriculture. Encapsulating therapeutic molecules within nanostructures such as micelles, liposomes, and polymersomes can improve the biodistribution of therapeutics, while minimizing systemic toxicity. ${ }^{309,310}$ Similarly in agriculture, encapsulated fertilizer nutrients can be protected from the environment, limiting their release to the environment. ${ }^{83}$ Responsive materials can give greater control over cargo delivery, as discussed in section 1.2. Due to the inherent hydrophilicity and predictable hybridization of DNA, it has been incorporated into these self-assembling nanostructures. The conjugation of a hydrophobic tail to DNA molecules creates amphipathic molecules that can form micelles, liposomes, and polymersomes. The structure of DNA can be exploited to make these nanostructures respond to environmental stimuli, such as complement strands, or $\mathrm{pH}$ for example.

Responsive DNA-lipid conjugate polymersomes have been created that could release a cargo in response to their complement DNA strand. Dentinger et al attached a tetradecyl hydrophobic tail to a short piece of DNA, resulting in amphiphilic conjugates. ${ }^{311}$ These conjugates formed spherical vesicle structures which could take up hydrophobic dyes. Upon addition of the complement strand, these hydrophobic dyes were released. More recently, 
Pokholenko et al. synthesized similar lipid oligonucleotide conjugates (LONs) using two or three hydrophobic chains. ${ }^{312}$ These conjugates formed micellar aggregates in which they could load the hydrophobic drug, paclitaxel. With the addition of the complement strand, the drug was released, creating a stimuli-responsive drug delivery system.

Yan et al. investigated the design of a DNA-nanostructure in which the size and structure could be controlled by $\mathrm{pH}^{313}$ The authors created a simple amphiphilic conjugate by covalently linking an 11-mer DNA sequence to a hydrophobic 18-carbon chain. The DNA sequence contained two cytosine-rich regions, allowing two conjugates to come together to form a dimer imotif structure under slightly acidic conditions, and return to its monomer structure at slightly basic conditions. This DNA structure switching in response to $\mathrm{pH}$ was translated to their micelles. At $\mathrm{pH} 4.5$, the DNA-conjugates self-assembled into multi-layered aggregates $\sim 50 \mathrm{~nm}$. At $\mathrm{pH} 9$, the DNA-conjugates formed micelles, which aggregated over time into larger $\sim 100 \mathrm{~nm}$ structures. More recently, Albert et al. also created a pH-responsive nanostructure using the reversible i-motif structure to trigger the release of cargo. ${ }^{314}$ The authors synthesized a DNAphenyleneethynylene (DNA-PE) amphiphilic conjugate using a cytosine-rich DNA strand capable of forming i-motifs at low $\mathrm{pH}$. At $\mathrm{pH} 7.3$, the DNA-PE conjugates were shown to selfassemble into DNAsome structures. At $\mathrm{pH}$ 5, the DNA could form i-motifs which caused the DNAsomes to break down into an entangled network and this effect was found to be reversible. The authors propose this DNAsome as a potential drug delivery vehicle, as they successfully demonstrated the encapsulation of the hydrophobic dye Nile red at $\mathrm{pH} 7.3$, and its subsequent release upon acidification to $\mathrm{pH} 5$. These examples demonstrate the ability to exploit DNA structure in order to create responsive materials. The incorporation of an affinity ligand such as an aptamer within these systems could make these materials respond to biomolecules. 


\subsubsection{Incorporation of aptamers into nanostructures}

While the conjugation of aptamers to the surface of nanoparticles and liposomes can facilitate delivery to target sites, their incorporation into the nanomaterial could make these structures responsive to biomolecules. Aptamers have been incorporated into a variety of smart materials, discussed in section 1.2, creating materials that can not only bind to a target of interest, but also respond to it. Aptamers incorporated into nanostructures such as polymersomes could allow these structures to bind a target of interest, as well as facilitate the delivery of a cargo. ${ }^{310}$

The Tan group incorporated an aptamer into a micelle nanostructure to specifically detect and deliver cargo to cancer cells. ${ }^{315}$ The authors attached a lipid tail to the Ramos cell-binding aptamer (TDO5) through a PEG spacer. The aptamer-lipid conjugate self-assembled into spherical micelles with an average diameter of $68 \mathrm{~nm}$. Importantly, when the TDO5 aptamer was incorporated into the micelle nanostructures, an 80-fold increase in affinity was observed compared to the free aptamer. The aptamer's incorporation into the micelle also facilitated internalization by the cells, which could be exploited for cargo delivery. The aptamer-micelle was able to recognize target cells after only 30 seconds with a 45 -fold enhancement in fluorescence for target cells over control cells. The authors also demonstrated this aptamermicelle's ability to bind target cells within a flow channel system that mimicked the bloodstream.

The same group created a nanostructure containing an aptamer switch probe capable of detecting intracellular ATP, which they termed switchable aptamer micelle flare (SAMF). ${ }^{316}$ Conjugates were synthesized which contained an aptamer probe, a PEG linker to a short piece of DNA which was complementary to the aptamer, and a diacyllipid tail. A fluorophore was attached to the 3' end, while a quencher was attached between the 5' end and the lipid tail. 
Hybridization of the aptamer probe to the short complement created a stem-loop structure resulting in the quenching of the fluorophore. Self-assembly of these amphiphilic conjugates created micelles $28-34 \mathrm{~nm}$ in diameter. The SAMFs showed specific binding to ATP with an almost 4-fold increase in fluorescence seen in response to ATP in vitro, and were able to show changes in ATP levels in vivo in real time. The incorporation of aptamers within nanostructures could make these materials bioresponsive which could be useful for controlled delivery applications.

\subsection{Materials and Methods}

\subsubsection{Chemicals and Reagents}

For DNA synthesis all amidites, reagents, and the 5'-DMS(O)MT-Amino-Modifier C6 were obtained from Glen Research, $1000 \AA$ CPG $1 \mu \mathrm{mol}$ scale columns were obtained from BioAutomation, and synthesis grade acetonitrile was obtained from VWR. 28\% ammonium hydroxide solution was obtained from Sigma-Aldrich (Oakville, ON, Canada). For the conjugation reaction, decanoic anhydride, dodecanoic anhydride, hexadecanoic anhydride, dimethylformamide (DMF), and diethylamine (DEA) were obtained from Sigma-Aldrich.

PAGE reagents: acrylamide/bis-acrylamide $40 \%$ solution, Tris, boric acid, EDTA and N,N,N',N'-tetramethylethylenediamine (TEMED), were obtained from BioShop Canada (Burlington, ON, Canada), while formamide and $3 \mathrm{kDa}$ cutoff Amicon Ultra centrifugal filter columns were obtained from Sigma-Aldrich.

For polymersome synthesis, Whatman nucleopore track-etched membranes with $0.1 \mu \mathrm{m}$ pores were obtained from Sigma-Aldrich, and filter supports were obtained from Avanti Polar Lipids (Alabaster, Alabama). Carbon film on 300 mesh copper grids (CF300-Cu) were obtained 
from Electron Microscopy Sciences (Hatfield, USA). For the stability and target-binding experiments, Sulforhodamine B (SB) and as well as potassium chloride $(\mathrm{KCl})$ were obtained from Sigma-Aldrich, while sodium chloride $(\mathrm{NaCl})$ was obtained from BioShop Canada. Regenerated cellulose membranes with a molecular-weight cut-off of $1000 \mathrm{Da}$ were obtained from Harvard Apparatus (Holliston, USA).

\subsubsection{Synthesis of aptamer-lipid conjugates}

The sulforhodamine B aptamer (SA; 5'NCCGGCCTAGGGTGGGAGGGAGGGGGCCGG-3') and a random oligonucleotide (RO; 5'NGACCTATGATAGCATCAGTCGCATCAGTC-3'), where N represents the 5'-DMS(O)MTAmino-Modifier C6 were synthesized using a BioAutomation Corporation MerMade 6 oligonucleotide synthesizer using standard phosphoramidite chemistry, without cleaving the final protecting group. Each column was washed with $300 \mu \mathrm{L}$ of acetonitrile, followed by 3 washes with $300 \mu \mathrm{L}$ of $10 \%$ DEA in acetonitrile, and 2 washes with $200 \mu \mathrm{L}$ of acetonitrile. The 5'DMS(O)MT protecting group was then removed using three additions of $300 \mu \mathrm{L}$ of Deblocking Mix (Glen Research), followed by two washes with $300 \mu \mathrm{L}$ of acetonitrile. Saturated anhydride solutions were made by dissolving decanoic, dodecanoic, or hexadecanoic anhydrides in DMF and heating to $35^{\circ} \mathrm{C}$ until dissolution ceased. The column beads were transferred to $1.5 \mathrm{~mL}$ microfuge tubes, and $1 \mathrm{~mL}$ of the anhydride solution was added. The tubes were incubated at $30^{\circ} \mathrm{C}$ for 48 hours on a rotary incubator.

The beads were then centrifuged at $14000 \times \mathrm{g}$ for 5 minutes and excess anhydride solution was discarded. The beads were washed twice using $1 \mathrm{~mL}$ of acetonitrile, discarding the supernatant. The aptamer-lipid conjugates were cleaved from the beads using $1 \mathrm{~mL}$ of 
ammonium hydroxide, and the samples were incubated at $32^{\circ} \mathrm{C}$ for 48 hours. The beads were centrifuged at $14000 \times \mathrm{g}$ for 10 minutes, collecting the supernatant. The beads were washed with $1 \mathrm{~mL} \mathrm{diH} \mathrm{H}_{2} \mathrm{O}$, collecting the supernatant. The supernatant was dried down using a Savant AE2010 SpeedVac on cryopump. The aptamer-lipid conjugates were resuspended in $\mathrm{diH}_{2} \mathrm{O}$, quantified using UV-Vis spectrophotometry, and their synthesis was confirmed using mass spectrometry.

\subsubsection{Synthesis of aptamer-polymersomes}

In a $5 \mathrm{~mL}$ glass vial, $40 \mathrm{nmol}$ of the aptamer-lipid conjugate was dried down using argon. The glass vial was set up using a retort stand and clamps, such that the bottom of the glass vial was submerged under the water line in a Symphony bath sonicator (VWR). $500 \mu \mathrm{L}$ of $\mathrm{diH}_{2} \mathrm{O}$ were added to the glass vial, and the sample was sonicated for 2 hours at $69^{\circ} \mathrm{C}$. The aptamerlipid sample was then transferred to a $500 \mu \mathrm{L}$ glass Gas Tight Syringe (Avanti Polar Lipids Inc.) and passed through a Whatman nucleopore track-etched membrane with $0.1 \mu \mathrm{m}$ pore size, into another glass syringe, using a Mini-Extruder (Avanti Polar Lipids Inc.) This sample was referred to as $(<100 \mathrm{~nm}) .500 \mu \mathrm{L}$ of $\mathrm{diH}_{2} \mathrm{O}$ was passed back from the second glass syringe, through the membrane, and into the original glass syringe in order to collect any aptamer-lipid polymersomes that did not pass through the membrane. This sample was referred to as $(>100 \mathrm{~nm})$.

\subsubsection{Imaging aptamer-polymersomes using TEM}

To prepare TEM grids, $10 \mu \mathrm{L}$ of each sample was deposited on a TEM grid (carbon film on 300 mesh copper grid). The sample was left to sit at room temperature for 10 minutes. The drop was aspirated off the TEM grid, and the grids were allowed to dry at least 2 hours before imaging. TEM imaging was done using a FEI Tecnai G2 F20 Transmission Electron Microscope (Hillsboro, USA) equipped with a Gatan ORIUS TEM CCD Camera (Pleasanton, USA). Polymersome size was measured using AmScope Toupview Camera software, by measuring the 
diameter of each polymersome structure. For irregular shaped structures, the longest diameter was taken. Histograms were generated using Logger Pro software (Vernier).

\subsubsection{Studying effects of carbon chain length on polymersome stability}

Aptamer-lipid conjugates were synthesized as described in section 4.4.2 using decanoic anhydride, dodecanoic anhydride, and hexadecanoic anhydride to generate sulforhodamine B aptamer conjugates with a 10-carbon, 12-carbon, and 16-carbon tail, respectively. Each of these aptamer conjugates was used to synthesize polymersomes as described in section 4.4.3. Immediately after polymersome synthesis, TEM grids were prepared using the $<100 \mathrm{~nm}$ and $>$ $100 \mathrm{~nm}$ samples for each aptamer-conjugate, representing the "Day 1" time point. New grids were prepared after 48 hours ("Day 3" time point), and 9 days after initial polymersome synthesis ("Day 9" time point). The polymersome samples were stored at $4^{\circ} \mathrm{C}$ between grid preparations.

\subsubsection{Examining target-binding using fluorescence enhancement}

SA-conjugate and RO-conjugate were prepared to $20 \mu \mathrm{M}$ each in buffer (10 mM Tris$\mathrm{HCl}, 100 \mathrm{mM} \mathrm{KCl}, 50 \mathrm{mM} \mathrm{NaCl}, \mathrm{pH}$ 7.45), and a $10 \mu \mathrm{L}$ sample of each was sent to 2 bind. A 1 $\mathrm{mL}$ solution of $1 \mathrm{mM}$ sulforhodamine B was prepared in the same buffer, and sent to 2 bind for analysis by fluorescence enhancement. In these experiments, the target dye was monitored after incubation with a range of DNA-conjugate concentrations. The SA- and RO-conjugates were heated to $90^{\circ} \mathrm{C}$ and allowed to cool to room temperature prior to incubation with SB. 2bind examined a range of DNA concentrations, from $\sim 150 \mathrm{pM}$ to $5 \mu \mathrm{M}$ mixed with $100 \mathrm{nM}$ sulforhodamine B, at $650 \mathrm{~nm}$ using a Monolith NT.115 Pico at $25^{\circ} \mathrm{C}$, with $70 \%$ LED power and 0.8 Laser power. 


\subsubsection{Examining target-binding using equilibrium dialysis}

Micro-equilibrium dialysis was used to estimate dissociation constants of the aptamerconjugates and aptamer-polymersomes. The aptamer-polymersomes were synthesized as described in section 4.4.3, and quantified directly after extrusion. A $300 \mu \mathrm{L}$ solution of $10 \mu \mathrm{M}$ aptamer-polymersome, $17.5 \mu \mathrm{M}$ sulforhodamine $\mathrm{B}$, and $0.1 \mathrm{M} \mathrm{KCl}$ was placed in the loading chamber of the Fast Micro-Equilibrium Dialyzer (Harvard Apparatus, Holliston, USA). A 100 $\mu \mathrm{L}$ sample of this solution was set aside for UV-Vis analysis. The receiving chamber contained $300 \mu \mathrm{L}$ of $0.1 \mathrm{M} \mathrm{KCl}$, and a regenerated cellulose membrane with a molecular-weight cut-off of $1000 \mathrm{Da}$ (Harvard Apparatus) was used. The dialyzers were incubated on a shaker at room temperature for 48 hours. Following the incubation, the absorbance of sulforhodamine B in the receiving chamber was analyzed by UV-Vis (peak at $564 \mathrm{~nm}$ ), and compared to its absorbance in the original sample loaded. The fraction of bound sulforhodamine $\mathrm{B}(f)$ was calculated as $f=$ $\left(S B_{T O T}-S B_{R}\right) / S B_{T O T}$, where $S B_{T O T}$ represented the absorbance of SB originally loaded into the dialyzer, and $S B_{R}$ represented the absorbance of SB recovered from the receiving chamber of the dialyzer. The dissociation constant $\left(\mathrm{K}_{\mathrm{d}}\right)$ could then be estimated by $K_{d}=X /(\mathrm{f}-X)$, where $\mathrm{X}$ represented the aptamer concentration.

\subsubsection{Examining target-binding using fluorescence anisotropy}

The binding of SA-conjugates and polymersomes to sulforhorhodamine B was examined using fluorescence anisotropy. Solutions of SA- and RO-conjugates (20 $\mu \mathrm{M}$ in buffer: $10 \mathrm{mM}$ Tris- $\mathrm{HCl}, 100 \mathrm{mM} \mathrm{KCl}, 50 \mathrm{mM} \mathrm{NaCl}, \mathrm{pH} 7.45)$ were heated to $90^{\circ} \mathrm{C}$ for 5 minutes, and cooled to room temperature, before addition of SB to final concentration of $500 \mathrm{nM}$. The conjugates were incubated with target for one hour prior to taking anisotropy measurements using a FluoroLog 3 equipped with polarizers (Horiba Jobin Yvon: Edison, NJ, USA). Anisotropy 
measurements were taken using emission and excitation wavelengths of $556 \mathrm{~nm}$ and $582 \mathrm{~nm}$, respectively, with $5 \mathrm{~nm}$ slit widths. Six measurements were taken for each sample, and the average value was plotted.

To determine an apparent dissociation constant for SA-conjugates, a range of SAconjugate concentrations (from 0.1 to $20 \mu \mathrm{M}$ ) in buffer were mixed with SB to a final SB concentration of $500 \mathrm{nM}$, incubated for an hour at room temperature, and measured as previously described. The average of six anisotropy measurements was plotted against DNA concentration, and the standard curve analysis feature of SigmaPlot (Version 10.0: Systat Software, Inc.) was used to fit the anisotropy data, which used the equation:

$y=\min +(\max -\min ) /\left(1+(x / E C 50)^{\wedge}\right.$ Hillslope $)$ where $\mathrm{y}=$ anisotropy, $\mathrm{x}=\mathrm{SA}-$ conjugate concentration, $\min =$ minimum anisotropy value, $\max =$ maximum anisotropy value, EC50 is the point of inflection of the curve and was used as an estimation of the apparent $\mathrm{K}_{\mathrm{d}}$, and Hillslope corresponds to the steepness of the curve at its inflection point.

To examine the binding of SA-polymersomes to SB compared to control ROpolymersomes, the DNA-polymersomes were synthesized as previously described. Immediately following their synthesis and extrusion, a range of DNA-polymersome concentrations (from 0.1 to $28 \mu \mathrm{M}$ ) in buffer were mixed with SB to a final SB concentration of $500 \mathrm{nM}$, incubated for an hour at room temperature, and measured as previously described. The average of six anisotropy measurements was plotted against DNA concentration. 


\subsection{Results and Discussion}

\subsubsection{Design and synthesis of aptamer-lipid conjugates}

Many of the DNA-lipid conjugates described in section 4.3 involved the multi-step synthesis of lipid-conjugated phosphoramidites. ${ }^{312-315}$ In order to investigate the incorporation of aptamers within nanostructures, a simpler system was designed using an existing aptamer for the dye sulforhodamine B. This sulforhodamine B aptamer had been used as a model system to examine aptamer incorporation into polyelectrolyte films and microcapsules and was shown to maintain its target-binding ability in these applications. Before incorporating the exudate-binding aptamers selected in Chapter 2 into delivery vehicles, a novel aptamer-lipid polymersome was investigated using the sulforhodamine B aptamer as a model system.

Adding a hydrophobic tail to DNA molecules has been shown to create amphiphilic molecules that can self-assemble into polymersome structures, which could be useful for targeted delivery and controlled release applications. The sulforhodamine B aptamer was synthesized with a 5' amine modification, allowing it to react with dodecanoic anhydride. This reaction added a 12-carbon hydrophobic tail to the aptamer, which has been shown to facilitate the selfassembly of amphiphilic molecules into polymersome structures. ${ }^{317}$ The design of this aptamerlipid conjugate is shown in Figure 4.1, and its synthesis was confirmed using mass spectrometry, shown in Figure 4.2.

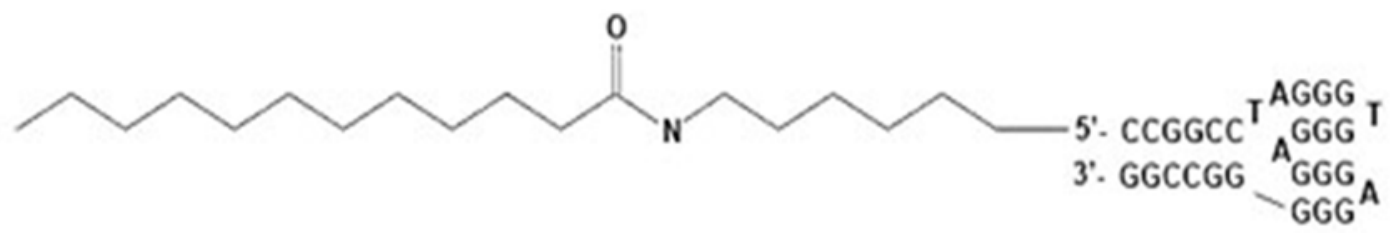

Figure 4.1: Amphiphilic aptamer-lipid conjugate showing the hydrophilic sulforhodamine $B$ aptamer head, and hydrophobic 12-carbon tail. ${ }^{318}$ 


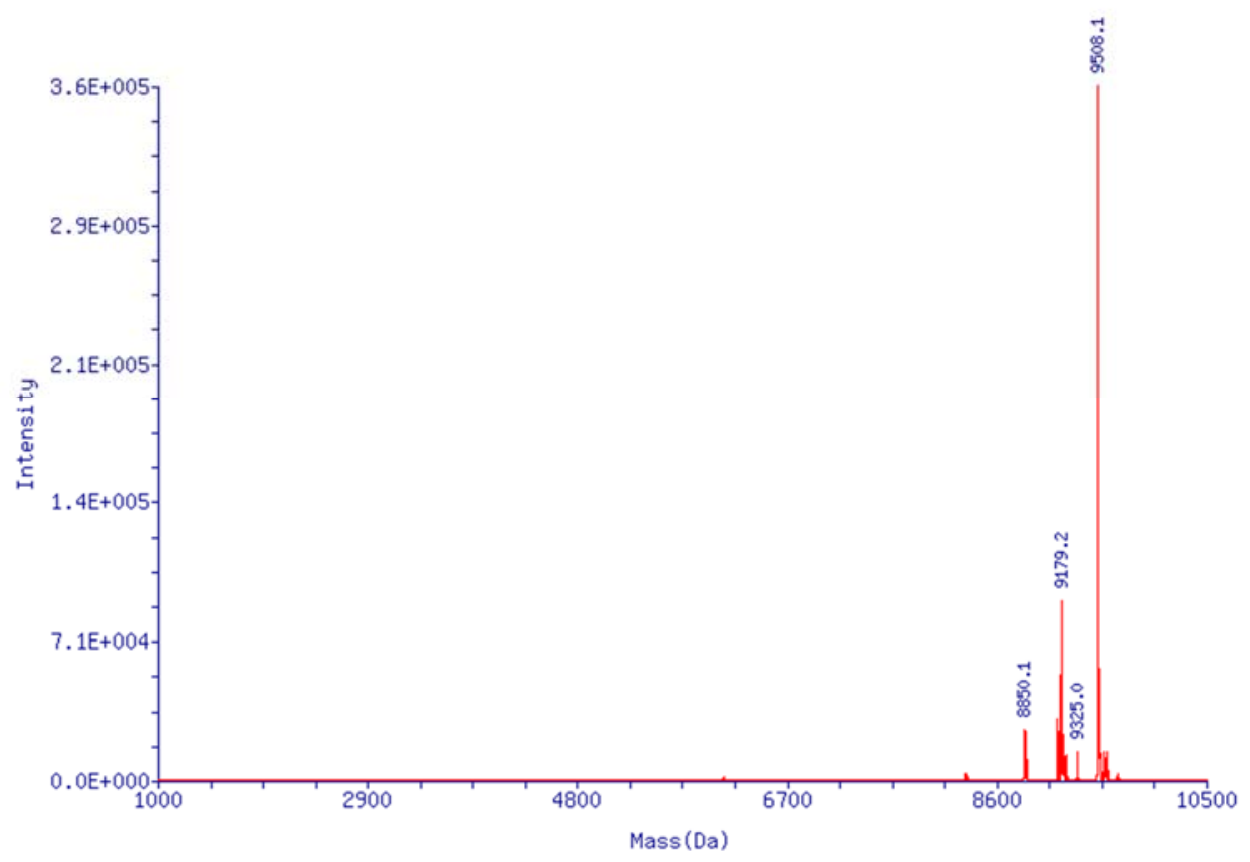

Figure 4.2: The synthesis of the sulforhodamine B aptamer-conjugate (expected mass 9509 Da) was confirmed by electrospray ionization liquid chromatography mass spectrometry (ESI/LC/MS) performed by Novatia (Newtown, USA).

The addition of the 12-carbon chain created an aptamer-lipid conjugate with a

hydrophilic aptamer head, and a hydrophobic lipid tail. To generate nanostructures using these aptamer-lipid conjugates, the thin-film hydration method was used, as it is one of the simplest and most common methods for liposome synthesis. ${ }^{319}$ This method is shown in Figure 4.3. The aptamer-conjugates were dried in a glass vial, and rehydrated with sonication to encourage the formation of polymersomes. 


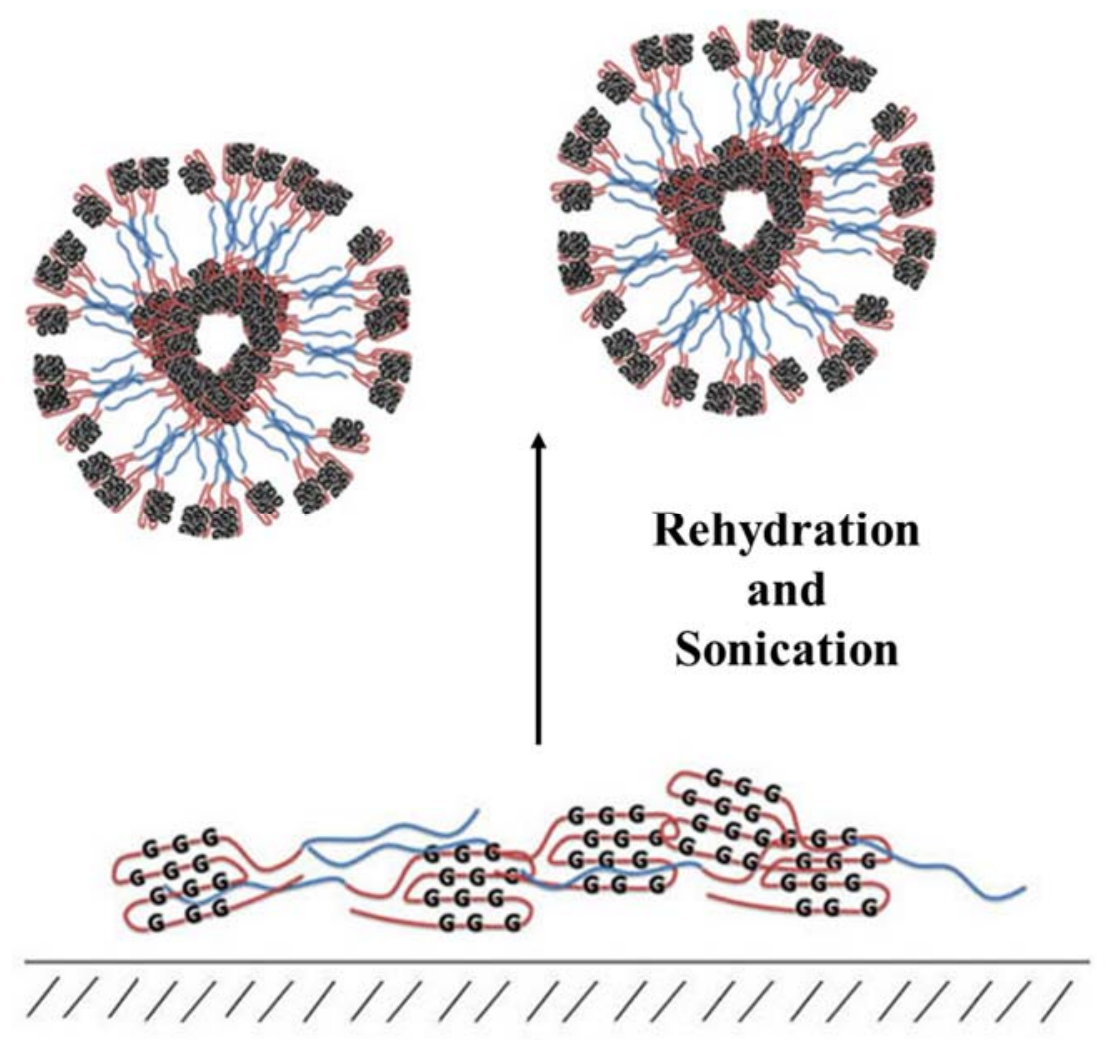

Figure 4.3: Aptamer-polymersomes were formed using the thin-film hydration method. Aptamer-conjugates were dried in a glass vial. This thin film was resuspended in water, sonicated at $69^{\circ} \mathrm{C}$, and extruded through $100 \mathrm{~nm}$ pores. ${ }^{318}$

This method could generate polymersomes of varying morphologies, and is therefore often paired with a sonication and an extrusion step to generate polymersomes of a desired size. As discussed in section 1.3.2, many aspects of plant biology are on the nanoscale, including pores and transport channels. ${ }^{72}$ Creating a fertilizer on the nanoscale could facilitate its uptake by plants. ${ }^{70,75}$ With this goal in mind, extrusion was performed using a filter containing $100 \mathrm{~nm}$ pores, and the resulting structures were examined.

\subsubsection{Effect of extrusion on aptamer-polymersome size}

The thin-film hydration method of producing polymersomes typically generates large, multilamellar vesicles, which can be made smaller and more uniform through sonication and extrusion techniques. ${ }^{320}$ The sonication of polymersome suspensions can produce small, 
unilamellar vesicles that are typically $15-50 \mathrm{~nm}$ in diameter. ${ }^{320}$ The sulforhodamine B aptamerlipid conjugates were dried in a glass vial, rehydrated in water, and sonicated for two hours. With the goal of creating polymersomes on the nanoscale, they were then subjected to extrusion through $100 \mathrm{~nm}$ pores. This extrusion process divided the polymersomes into two groups by size, those that passed through the $100 \mathrm{~nm}$ pores, and those that were too large to pass through the 100 $\mathrm{nm}$ pores. To examine these groups in greater detail, the polymersomes were deposited onto carbon-copper grids following extrusion, and imaged using TEM, shown in Figure 4.4. 

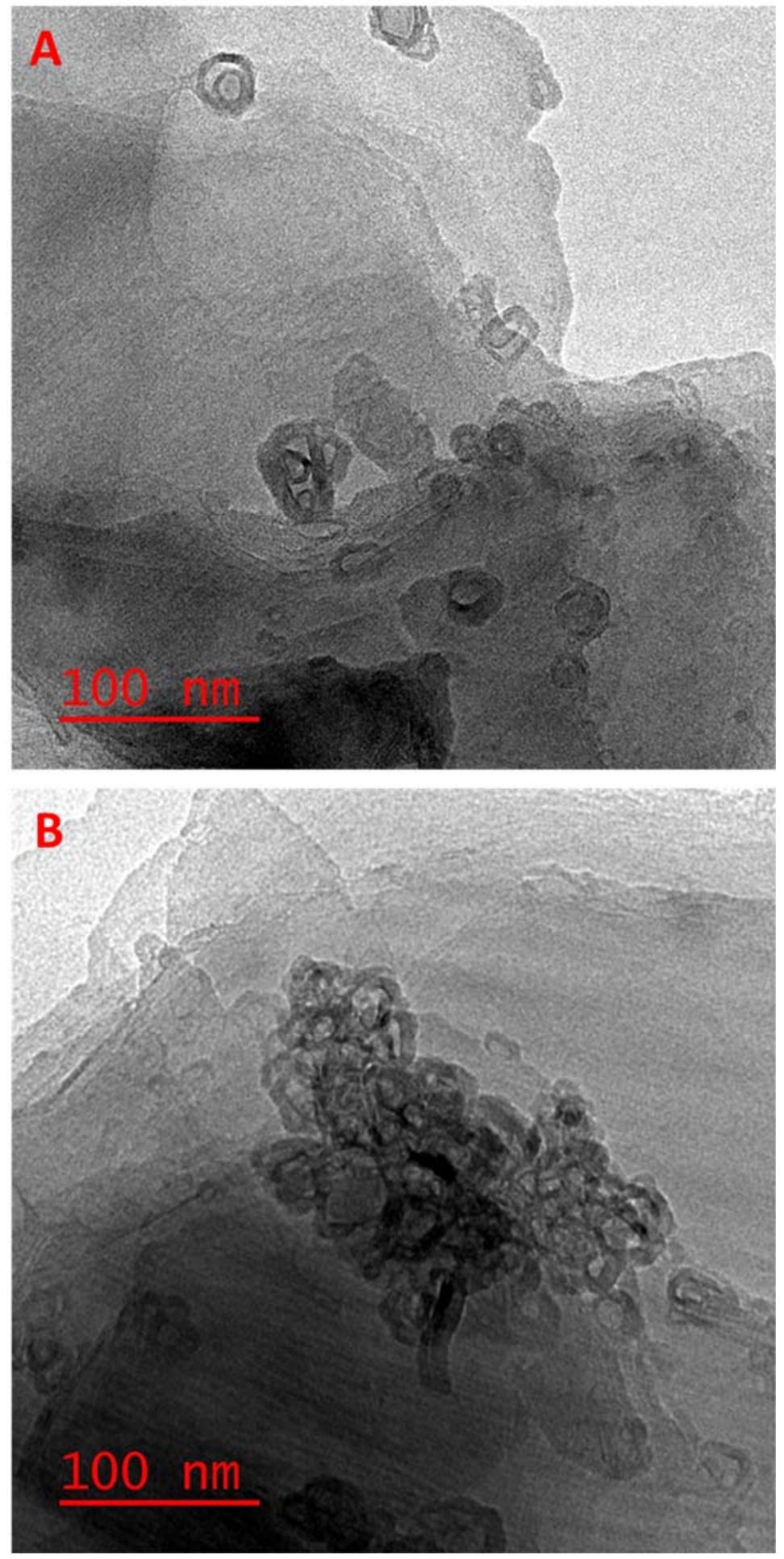

Figure 4.4: The morphology of sulforhodamine B aptamer (SA)-conjugates synthesized with 12-carbon chains, and extruded through a $100 \mathrm{~nm}$ filter was examined using transmission electron microscopy (TEM). (A) Aptamer-polymersomes which penetrated the $100 \mathrm{~nm}$ filter; (B). Aptamer-polymersomes which did not penetrate the $100 \mathrm{~nm}$ filter. 
It was originally hypothesized that multiple morphologies would be generated following the thin film hydration, allowing extrusion to separate larger nanostructures from smaller nanostructures. The aptamer-lipid conjugates were amphiphilic block-copolymers in which the hydrophilic aptamer head provided the bulk of the mass. Self-assembly in water of block copolymers such as these could have produced a compact micellar structure in which a small, hydrophobic core was surrounded by a thick, hydrophilic corona. ${ }^{321}$ Alternatively, a bilayer could have formed, with hydrophilic polymers facing both outward and inwards, creating a polymersome with a lipid-based membrane surrounding an aqueous core. ${ }^{321}$ In examining the TEM images shown in Figure 4.4, one predominant morphology was observed. Small polymersomes were observed in the sample that penetrated the $100 \mathrm{~nm}$ filter, as well as in the sample that did not pass through the filter during extrusion. Films can also be observed in the background, which are likely aptamer-lipid conjugates that did not dissolve and assemble into nanostructures during the thin-film hydration method, or which collapsed on the grid during the drying process. ${ }^{321}$ Rather than larger individual polymersomes, the sample which did not pass through the filter appeared to contain aggregates of small polymersomes. To examine whether there was a size difference of individual polymersomes as a result of extrusion, the polymersomes in each sample were measured, and the size distribution is shown in Figure 4.5. 

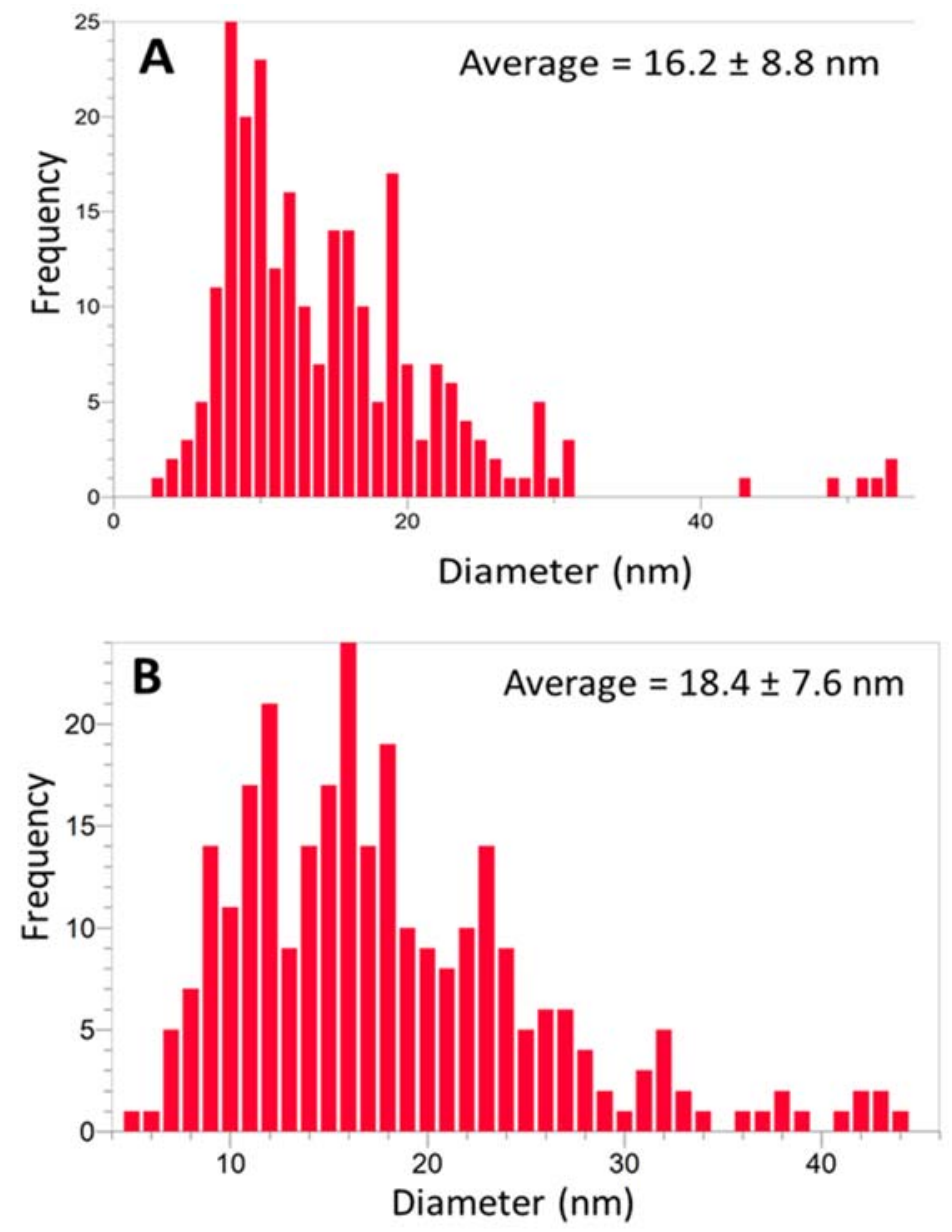

Figure 4.5: The size distribution of SA-polymersomes after extrusion through a filter with $100 \mathrm{~nm}$ pores was determined by measuring the TEM images using AmScope Toupview Camera software. (A) SA-polymersomes that penetrated the $100 \mathrm{~nm}$ filter; (B) SApolymersomes that did not pass through the $100 \mathrm{~nm}$ pores.

Both samples appeared to be polydisperse, containing polymersomes which ranged in size from 10-30 nm. The polymersomes that penetrated the $100 \mathrm{~nm}$ filter were found to be slightly smaller compared to those that did not pass through the filter, with an average diameter of $16.2 \pm 8.8 \mathrm{~nm}$ and $18.4 \pm 7.6 \mathrm{~nm}$, respectively. This could suggest a slight decrease in size was obtained by extruding through a $100 \mathrm{~nm}$ filter, however the presence of a few larger polymersomes which did not pass through the filter could be affecting the observed average. Figure 4.5 suggested that rather than separating larger polymersomes from smaller ones, the extrusion process separated aggregates of polymersomes into a more dispersed sample. The 
characterization of the size and target-binding ability of these polymersomes would be difficult using aggregated polymersomes, as the aggregate size could not be controlled. For all further characterization, the more dispersed aptamer-polymersome samples that penetrated the $100 \mathrm{~nm}$ pores during extrusion were used for analysis.

\subsubsection{Effect of carbon chain-length on aptamer-polymersome size}

The size of the aptamer-polymersomes could affect the stability, target-binding, and cargo loading. The ability to predictably tune the size of aptamer-polymersomes could be advantageous to control binding parameters and cargo-loading. Since the extrusion process seemed to disperse aggregated polymersomes rather than alter their individual size, the length of the hydrophobic tail was altered to investigate its effect on polymersome size. The reaction of dodecanoic anhydride with the amine-modified aptamer generated an aptamer-conjugate with a 12-carbon tail. Decanoic anhydride and hexadecanoic anhydride were reacted with the aminemodified aptamer to generate aptamer conjugates with a 10-carbon, and 16-carbon tail, respectively. Aptamer-polymersomes were prepared using the aptamer-conjugates with 10-, 12-, and 16-carbon hydrophobic tails to determine how tail length affected the polymersome size. TEM images and aptamer-polymersome diameter measurements are shown in Figure 4.6. 
10-Carbon chain
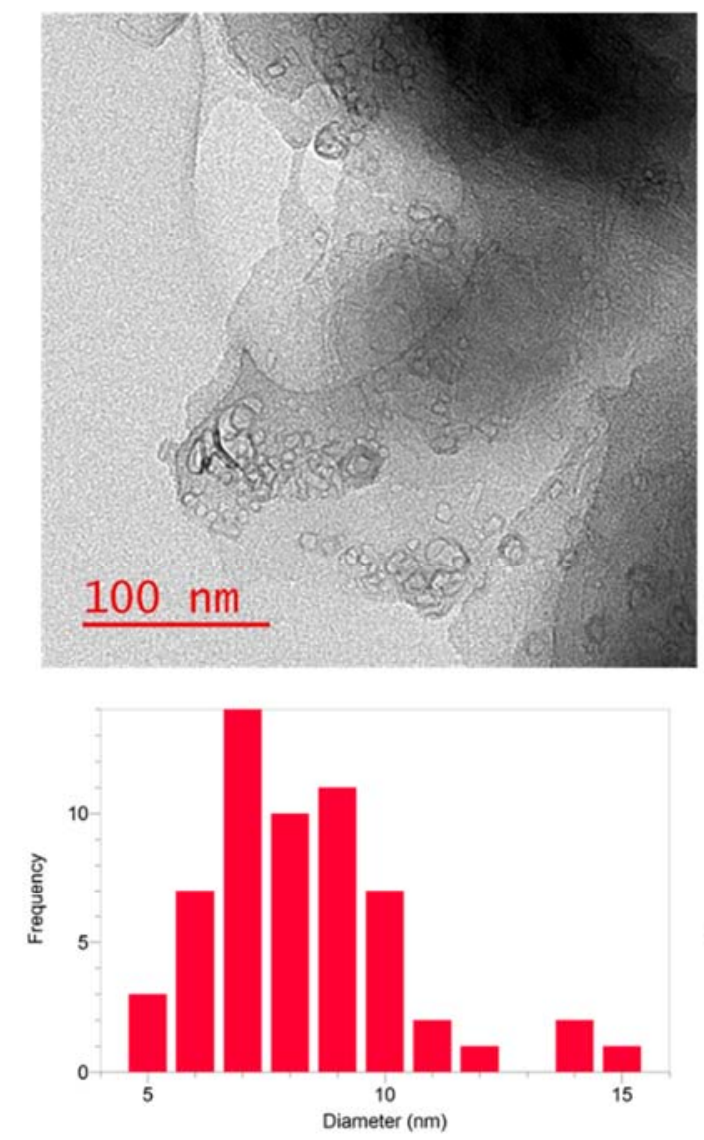

Average $=8.3 \pm 2.1 \mathrm{~nm}$
12-Carbon chain
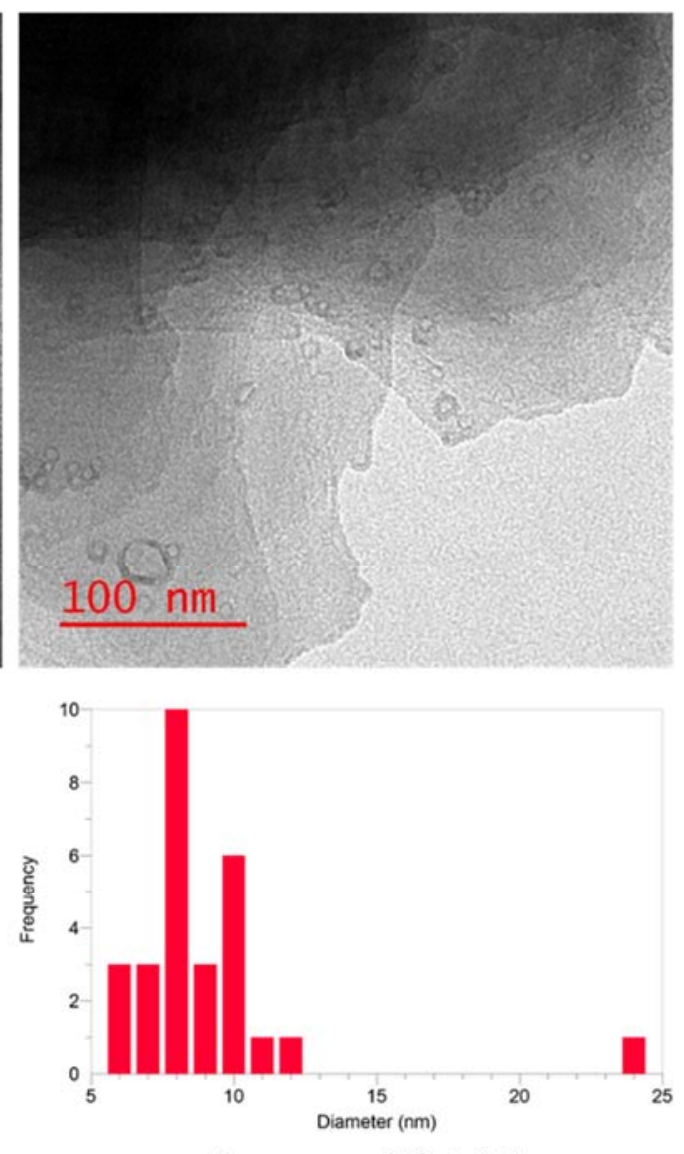

Average $=8.9 \pm 3.4 \mathrm{~nm}$
16-Carbon chain
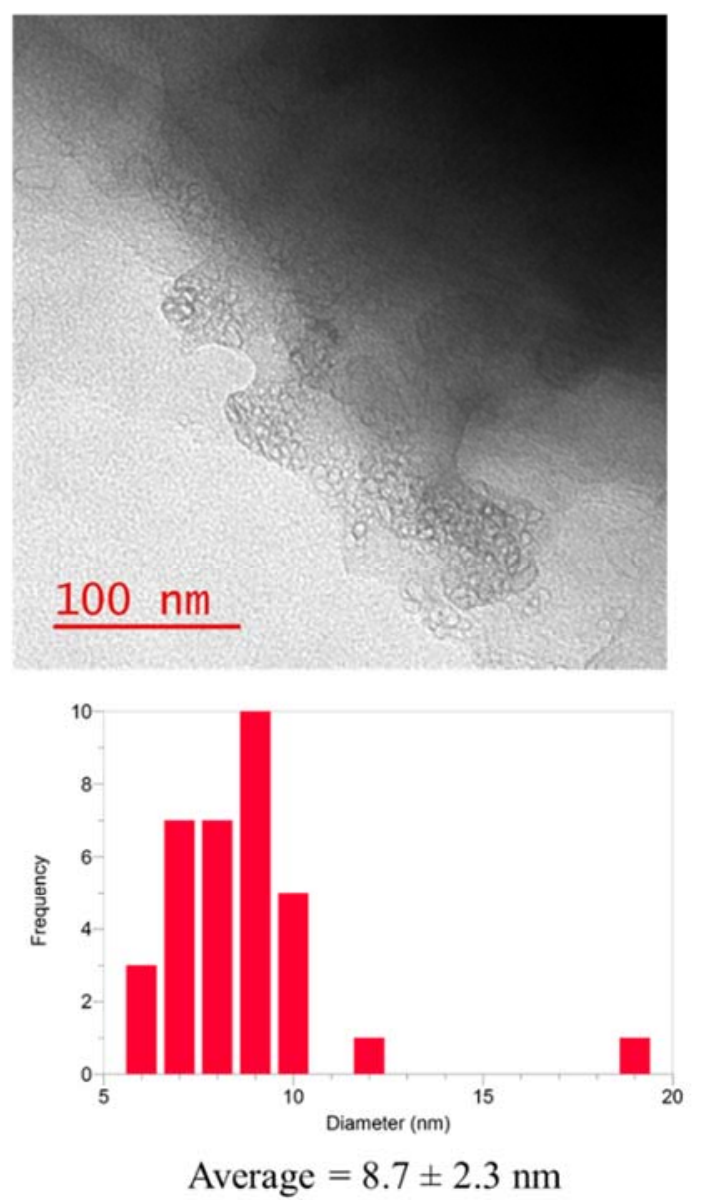

Figure 4.6: The effect of the aptamer-conjugate's hydrophobic tail length on polymersome size was examined using TEM. The top row shows the aptamer-polymersome morphology with 10-, 12-, and 16-carbon hydrophobic tails, while the bottom row shows the size distribution of the corresponding aptamer-polymersomes measured using AmScope Toupview Camera software. 
Synthesizing aptamer-polymersomes with 10-, 12-, and 16-carbon hydrophobic tails did not cause an observable change in the aptamer-polymersome morphology. The average diameters of the aptamer-polymersomes with 10-, 12-, and 16-carbon hydrophobic tails were 8.3 $\pm 2.1 \mathrm{~nm}, 8.9 \pm 3.4 \mathrm{~nm}$, and $8.7 \pm 2.3 \mathrm{~nm}$, respectively. These average diameters appear smaller than those observed in section 4.5.2, however this is likely due to a smaller sample size examined, and still fall within the standard deviation previously observed. The mass of the aptamer-conjugate was $9509 \mathrm{Da}$, with the sulforhodamine $\mathrm{B}$ aptamer alone having a mass of $9147 \mathrm{Da}$. Since the aptamer comprised such a large portion of the mass, it is likely that a small change from ten to sixteen carbon atoms in the hydrophobic tail was not enough to affect a change in polymersome morphology. If larger polymersomes were desired, longer lipid chains could be used. Aptamer-polymersomes synthesized with diacyl chains 18-carbons each have been used to generate polymersomes $28-34 \mathrm{~nm}$ in diameter for example. ${ }^{315,316}$ As our application aims to match a fertilizer to the nanoscale pores in plants, the small size of these aptamerpolymersomes was desirable.

\subsubsection{Effect of carbon-chain length on aptamer-polymersome stability}

In order to move aptamer-polymersomes into useful targeted-delivery applications, these structures would need to remain stable in solution. The leakage of encapsulated cargo molecules as well as the aggregation of polymersomes in solution are challenges that must be overcome for the widespread use of polymersome-based delivery tools. ${ }^{319}$ DNA-lipid conjugates synthesized with an 18-carbon lipid tail were shown to self-assemble into micelles $\sim 50 \mathrm{~nm}$ in size. The size of the DNA-micelles were examined over time and were found to more than double in size over 6 days. ${ }^{313}$ The aggregation of polymersomes over time would cause a non-uniform sample, and could cause the premature release of cargo molecules. The aptamer-polymersomes synthesized 
with 10-, 12-, and 16-carbon hydrophobic tails were kept at $4{ }^{\circ} \mathrm{C}$ in water, and sampled after 1,3 and 9 days to examine any changes in morphology over time. The resulting TEM images are shown in Figure 4.7 to Figure 4.9. 

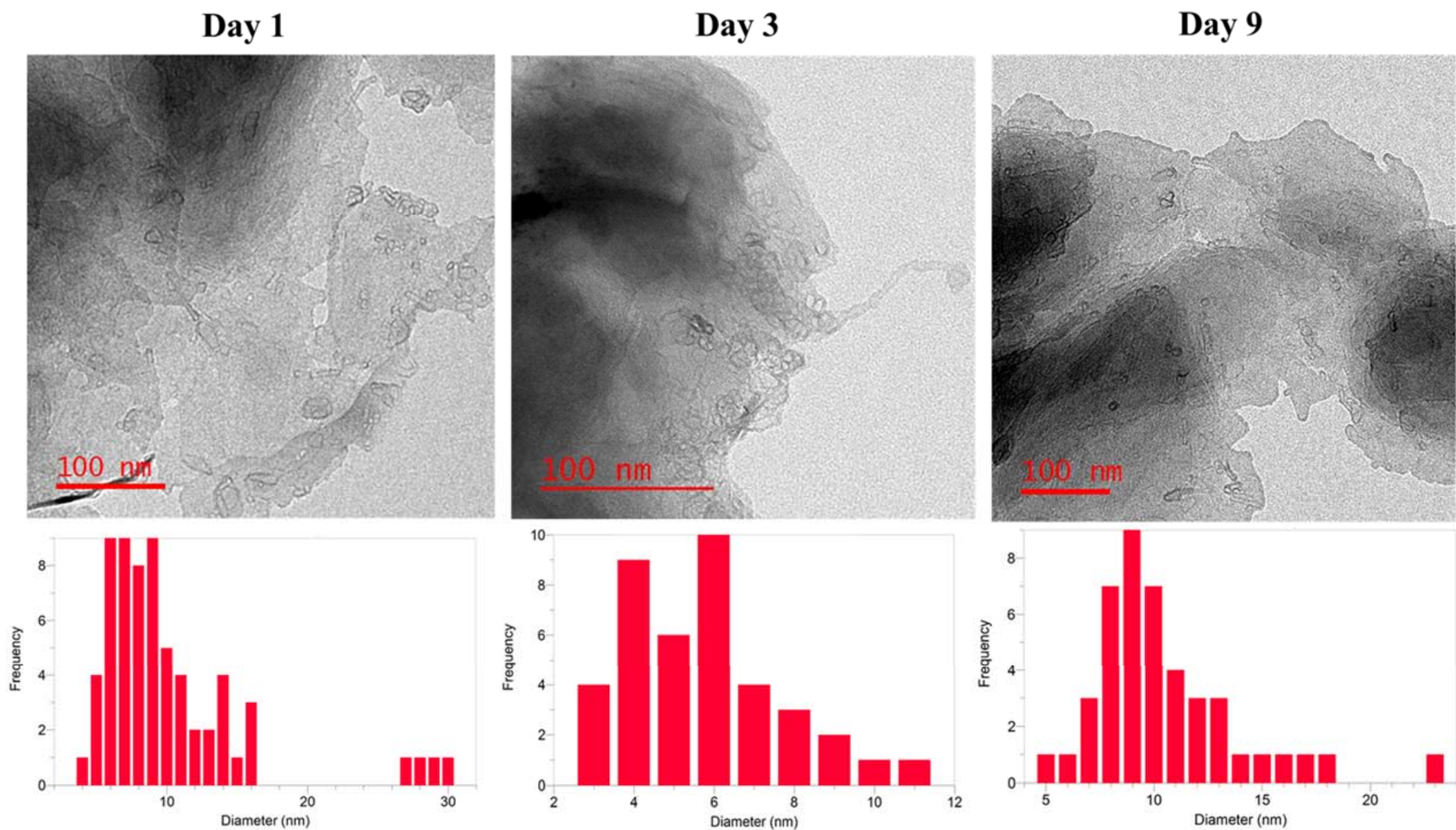

Average $=10.2 \pm 5.5 \mathrm{~nm}$

Average $=5.8 \pm 1.9 \mathrm{~nm}$

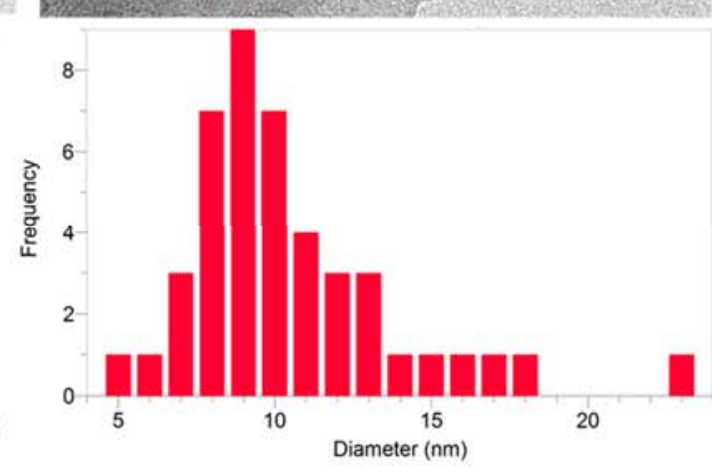

Average $=10.5 \pm 3.4 \mathrm{~nm}$

Figure 4.7: The stability of the aptamer-polymersomes synthesized with conjugates containing a 10-carbon hydrophobic tail was examined using TEM. The top row shows the aptamer-polymersome morphology after 1, 3, and 9 days of storage in water, while the bottom row shows the size distribution of the corresponding aptamer-polymersomes measured using AmScope Toupview Camera software. 

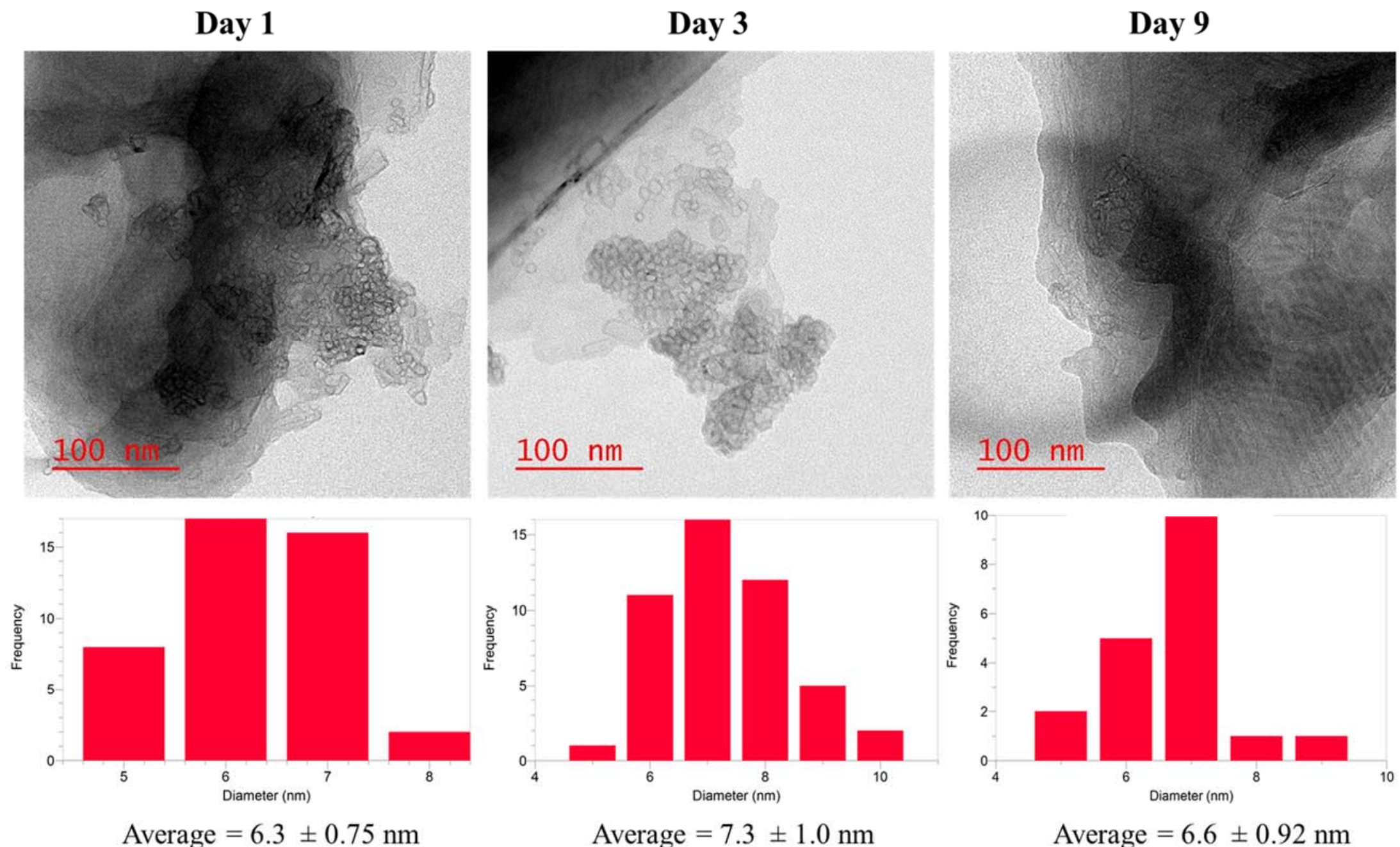

Average $=7.3 \pm 1.0 \mathrm{~nm}$

Average $=6.6 \pm 0.92 \mathrm{~nm}$

Figure 4.8: The stability of the aptamer-polymersomes synthesized with conjugates containing a 12-carbon hydrophobic tail was examined using TEM. The top row shows the aptamer-polymersome morphology after 1, 3, and 9 days of storage in water, while the bottom row shows the size distribution of the corresponding aptamer-polymersomes measured using AmScope Toupview Camera software. 

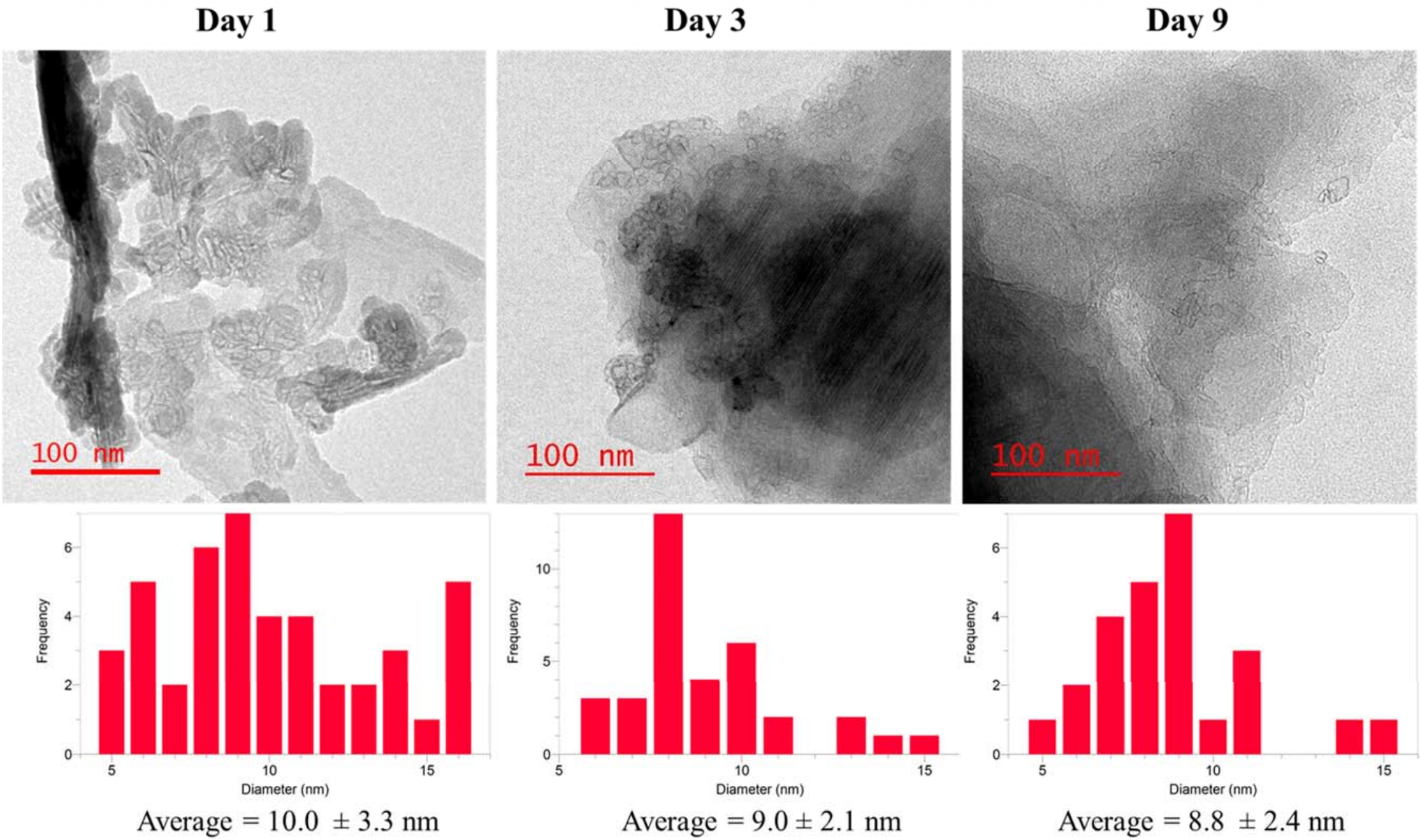

Figure 4.9: The stability of the aptamer-polymersomes synthesized with conjugates containing a 16-carbon hydrophobic tail was examined using TEM. The top row shows the aptamer-polymersome morphology after 1, 3, and 9 days of storage in water, while the bottom row shows the size distribution of the corresponding aptamer-polymersomes measured using AmScope Toupview Camera software. 
After the aptamer-polymersomes were stored for nine days at $4{ }^{\circ} \mathrm{C}$ in water, they could still be seen with TEM, shown in Figure 4.7 to Figure 4.9, which indicated that the polymersomes did not break down over their 9 day storage. The average diameters of the aptamer-polymersomes were measured from the TEM images, and no difference was found between the diameters measured on day 1 and on day 9 for any of the chain lengths (confirmed using one-way ANOVA). This finding suggested that the change in hydrophobic chain length from 10 to 16 carbon atoms did not affect the stability of the aptamer-polymersomes in solution. In addition, the morphology of these aptamer-polymersomes was consistent over at least 9 days. Since no difference in size or stability was observed by altering the hydrophobic chain length, the 12-carbon aptamer-conjugate was used to study target-binding.

\subsubsection{Target-binding interactions of aptamer-conjugates and aptamer-polymersomes}

\subsubsection{Synthesis of random-oligonucleotide-conjugates and polymersomes}

In order for the aptamer-conjugates and aptamer-polymersomes described herein to be utilized as targeted-delivery vehicles, it had to be confirmed that the aptamer was able to bind its target after its conjugation to a hydrophobic tail, and after its incorporation into polymersomes. To investigate the sulforhodamine B aptamer's ability to bind its target dye, a control conjugate had to be synthesized. A random oligonucleotide (RO) had been used in studies examining the sulforhodamine B aptamer's target-binding after incorporation into PEM films and microcapsules, and was found not to bind sulforhodamine B. ${ }^{247,250}$ This non-binding RO was used to synthesize a control DNA-lipid conjugate for target binding studies. The RO-conjugate was used to form polymersomes via the thin-film hydration method, followed by extrusion through $100 \mathrm{~nm}$ pores, as previously described. The morphology of the RO-polymersomes was examined by TEM, and is shown in Figure 4.10. 

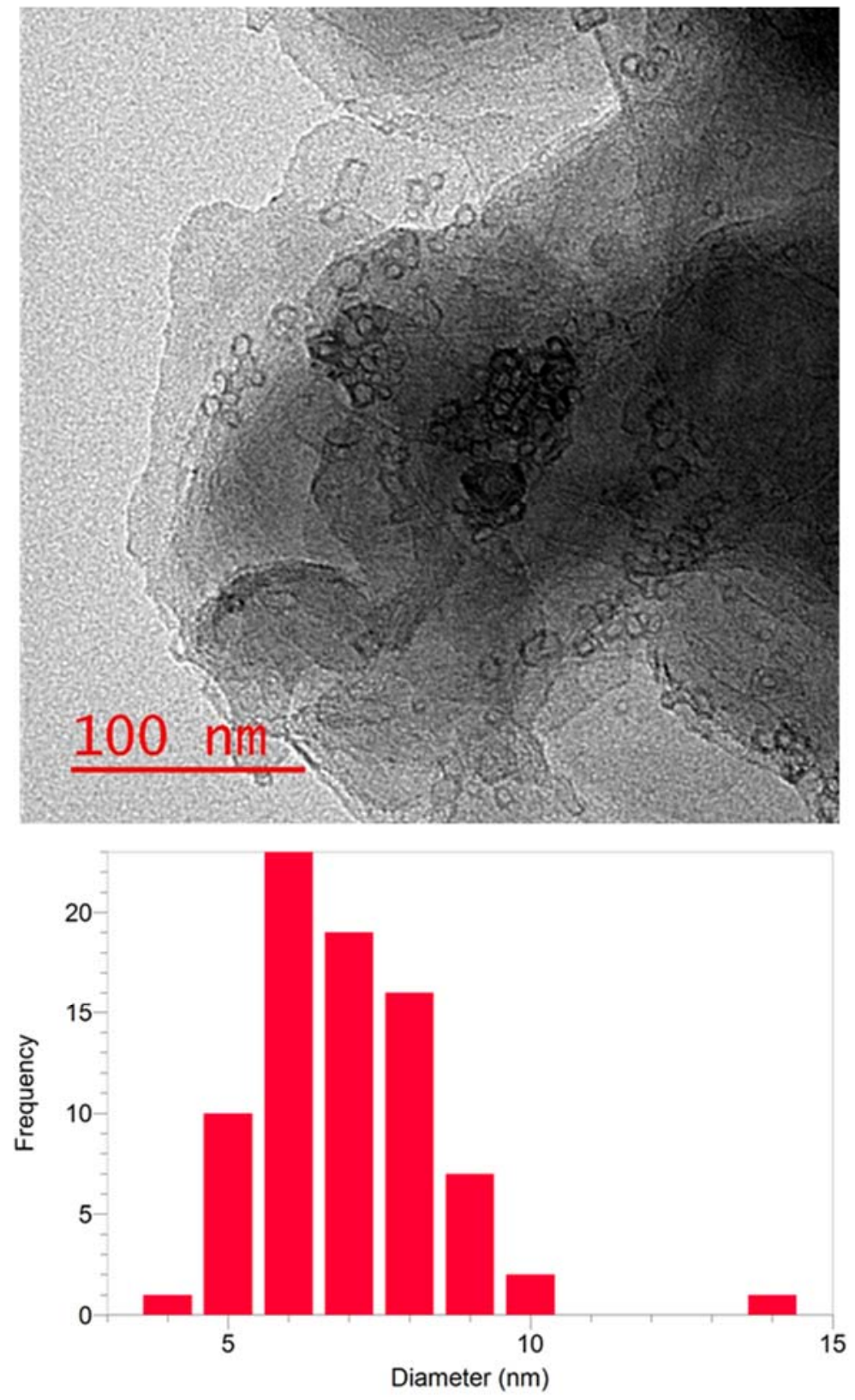

Average $=7.0 \pm 1.5 \mathrm{~nm}$

Figure 4.10: The morphology of polymersomes synthesized using a randomoligonucleotide-conjugate (RO-polymersomes) was examined using TEM (Top image). The RO-polymersomes were measured using AmScope Toupview Camera software (bottom image). 
The TEM image in Figure 4.10 shows the RO-polymersomes with the same morphology seen for the SA-polymersomes. The average diameter of the RO-polymersomes was $7.0 \pm 1.5$ $\mathrm{nm}$, which fell within the standard deviations seen for the diameters measured for SApolymersomes in Figure 4.6. These images indicated that the morphology observed for the SAand RO-polymersomes was unrelated to the specific DNA sequence (RO-conjugates were not predicted to form G-quadruplexes), and this system could be generalized to other aptamers. These RO-conjugates and RO-polymersomes were used during target-binding studies as a nonbinding control.

\subsubsection{Examining target-binding using fluorescence enhancement}

Microscale thermophoresis was used to determine the apparent dissociation constants for the L-serine-binding aptamers in solution (section 3.5.2). MST was investigated as an affinity test for the aptamer-conjugate as it was of interest to determine its affinity in solution as well. Since the SB target in this case was a dye, the fluorescence of a constant concentration of SB was monitored after incubation with a range of unmodified SA-conjugate concentrations. A couple of challenges arose in the attempt to use MST for this analysis. As the SA-conjugate DNA did not have a fluorescent modification, the target dye had to be measured instead. This was a challenge because the excitation and emission wavelengths for SB were $565 \mathrm{~nm}$ and 586 nm, respectively, which fell within a detection gap of the Monolith NT.115 Pico used by 2 bind. Using a high concentration of SB, the shoulder of its emission peak could be detected, and a DNA concentration-dependent fluorescence enhancement was observed. This fluorescence enhancement of SB with increasing SA-conjugate concentration was used to get an estimate of binding rather than thermophoresis, and is shown in Figure 4.11. 


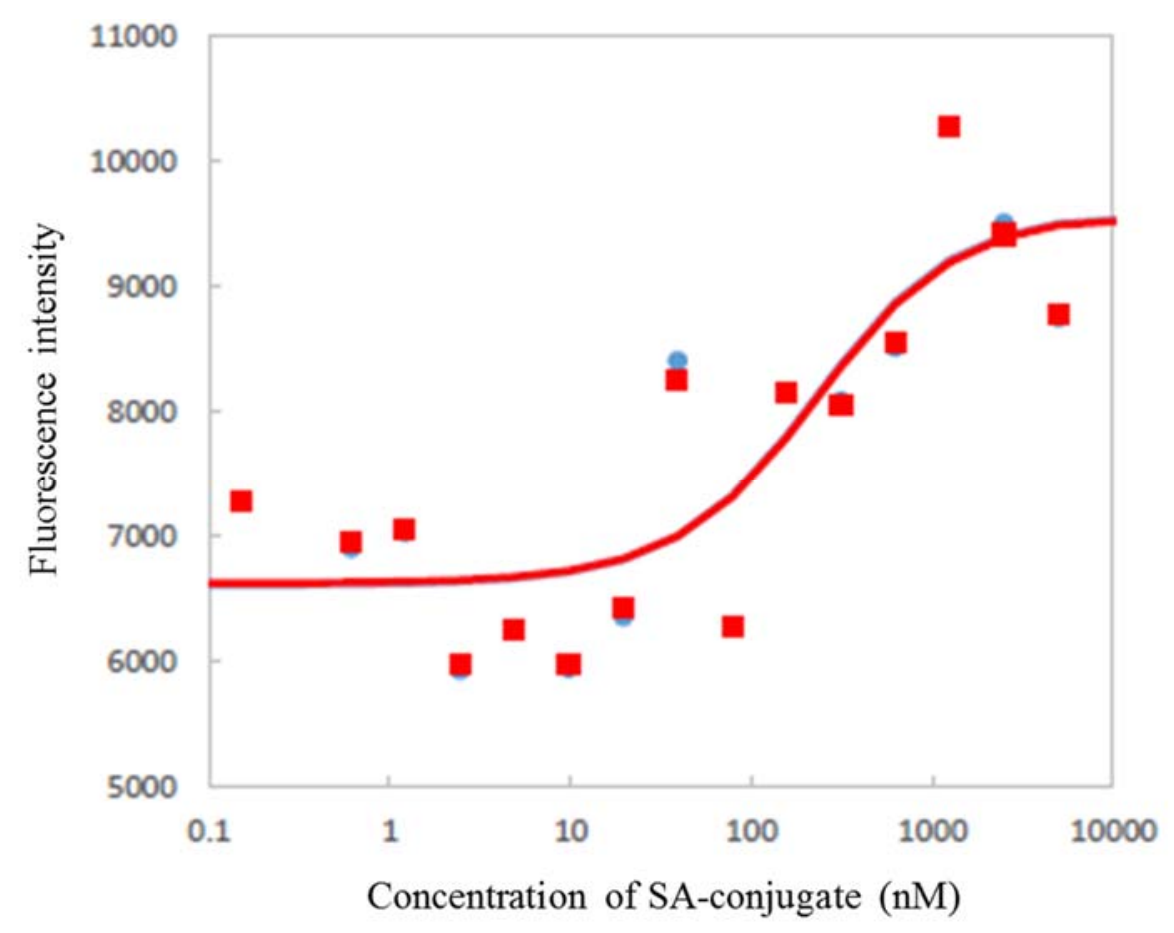

Figure 4.11: The interaction of the SA-conjugate with SB was examined using targetbinding specific fluorescence enhancement. The fluorescence intensity of SB (100 nM) was monitored at $650 \mathrm{~nm}$ over a range of $S A$-conjugate concentrations ( $150 \mathrm{pM}-5 \mu \mathrm{M})$.

Figure 4.11 shows a trend of increasing SB fluorescence with increasing SA-conjugate concentration. This same trend was not observed for the RO-conjugate. This trend suggested that the fluorescence of SB was enhanced upon binding to the SA-conjugate. The sulforhodamine B aptamer has been shown to form a G-quadruplex, and this structure has been shown to enhance the fluorescence of a variety of dye molecules upon binding. ${ }^{322,323}$ Using this fluorescence enhancement trend, a dissociation constant could be roughly estimated for the SA-conjugate to be between 100-1000 nM. No fluorescence enhancement was observed for the RO-conjugate, which was not predicted to form a G-quadruplex, but some non-specific interactions could be observed at high SB concentration. No dissociation constant could be inferred due to the high dye concentration required for measurement. This trend observed for the SA-conjugate provided 
some indication of target-binding, but other affinity methods were tested for a more quantitative $\mathrm{K}_{\mathrm{d}}$ determination.

\subsubsection{Examining target-binding using equilibrium dialysis}

In order to assess the binding of aptamers to their targets, several methods for quick and cost-effective screening have been proposed, such as equilibrium dialysis and affinity

chromatography. ${ }^{281}$ As sulforhodamine B is a visible dye and it was of interest to investigate the target-binding in solution, a one-point micro-equilibrium dialysis test was chosen to screen the aptamer-conjugates and polymersomes for target-binding. This dialysis screening method has been previously used with small molecules tyramine and ochratoxin A (OTA). ${ }^{267,281}$ For this screening, SA- and RO-conjugates and polymersomes (10 $\mu \mathrm{M}$ each) were mixed with $17.5 \mu \mathrm{M}$ $\mathrm{SB}$ in $100 \mathrm{mM} \mathrm{KCl}$, and dialyzed against $100 \mathrm{mM} \mathrm{KCl}$ for 48 hours before analyzing each chamber using UV-Vis spectroscopy. In a dialysis of SB on its own, after 48 hours there was an equal amount of SB on both sides of the dialysis membrane indicating that equilibrium had been reached. For target-binding to be observed, a higher proportion of SB would be expected to remain in the loading-chamber, bound to aptamer rather than penetrating the membrane. No binding could be observed for the DNA-conjugates or polymersomes using this screening method. When this test was used to screen aptamers binding both tyramine, and OTA, the quantification was performed using HPLC rather than UV-Vis spectroscopy. It is likely that with such a high concentration of SB, the UV-Vis quantification was not sensitive enough to detect small changes in SB concentration. McKeague et al. tested a series of OTA-binding aptamers using a variety of affinity tests. ${ }^{281}$ The authors found that no aptamer performed equally well in all tests. Only two of seven OTA-binding aptamers showed target-binding using equilibrium 
dialysis, leaving the authors to conclude that multiple screening methods should be performed to confirm aptamer-target binding.

\subsubsection{Examining target-binding using fluorescence anisotropy}

As discussed in section 3.3.2, the determination of apparent dissociation constants of aptamers for small molecule targets can be a challenge, and often requires that either the target, or the aptamer be fluorescently labeled. One such method is fluorescence anisotropy, which is commonly used to determine the apparent dissociation constants of aptamers for small-molecule targets, with high accuracy. ${ }^{281,282,294,324}$ As the model system described herein utilizes the dye sulforhodamine B as the target molecule, fluorescence anisotropy could be used. The anisotropy values obtained for a fluorescent molecule depend on its rotation in solution. A free molecule will rotate differently than an aptamer-bound molecule, resulting in a measurable change in anisotropy upon aptamer-target binding. Typically anisotropy values are small for a molecule

free in solution, and increase upon binding. ${ }^{324}$

To determine if the conjugation of a lipid tail to the sulforhodamine B aptamer affected its ability to bind its target, the binding of the SA-conjugates to SB was examined using fluorescence anisotropy, using RO-conjugates with SB and SB alone as controls. After the DNAconjugates were incubated with SB for one hour, anisotropy measurements were taken, and are shown in Figure 4.12. 


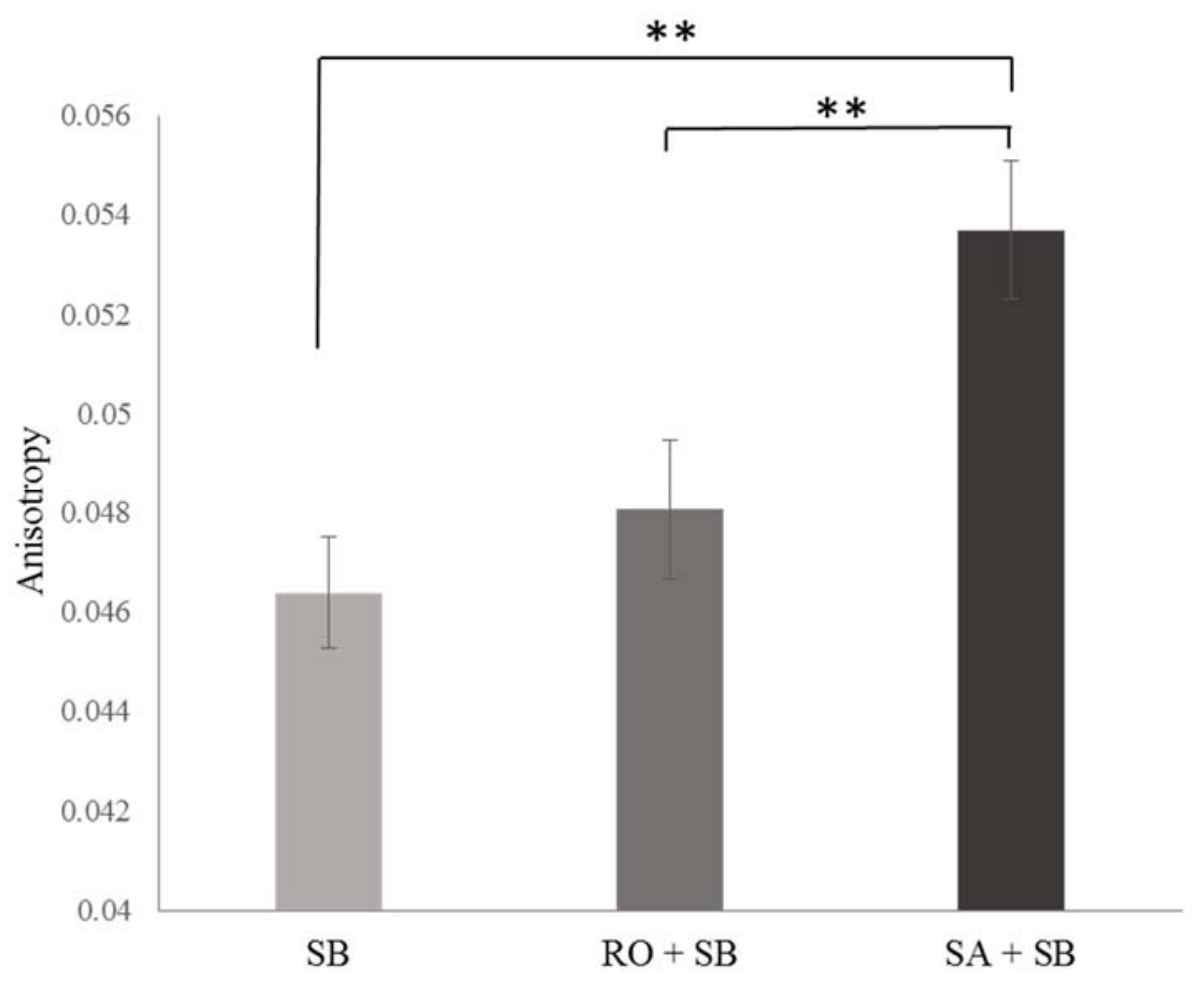

Figure 4.12: The binding of the sulforhodamine B aptamer-conjugate (SA) and of a random oligonucleotide conjugate (RO) to sulforhodamine $B$ (SB) was examined using fluorescence anisotropy. The DNA-conjugates $(20 \mu \mathrm{M})$ were incubated with SB (500 $\mathrm{nM})$ for one hour prior to taking measurements. Anisotropy values were measured with excitation and emission wavelengths of $556 \mathrm{~nm}$ and $582 \mathrm{~nm}$, respectively. Error bars represent the standard deviation of six replicates. A one-way ANOVA determined a statistical difference in anisotropy values for the SA-conjugate compared to the ROconjugate and the $S B(P<0.01)$.

Figure 4.12 shows that the anisotropy values increased when sulforhodamine B was incubated with the SA-conjugate, which suggested the SA-conjugate was able to bind SB in solution. Statistical analysis (one-way ANOVA) showed that there was no difference between the anisotropy values obtained for $\mathrm{SB}$ in solution and the RO-conjugate with $\mathrm{SB}$, but there was a significant difference between the anisotropy values of SA-conjugate with SB and both the ROconjugate with $\mathrm{SB}$ and $\mathrm{SB}$ alone $(\mathrm{P}<0.01)$. These results suggested that the $\mathrm{RO}$-conjugate did not bind SB, as the anisotropy values obtained when it was mixed with SB were not different from the dye alone. The anisotropy values obtained for the SA-conjugate with SB were 
significantly higher than both controls, suggesting that the aptamer was able to bind SB in solution even when it was conjugated to a 12-carbon hydrophobic tail. To get an estimate of the apparent dissociation constant of the aptamer-conjugate to $\mathrm{SB}$, a range of aptamer-conjugate concentrations were incubated with $500 \mathrm{nM} \mathrm{SB}$ for one hour, and their anisotropy values were measured. The average of six replicate anisotropy values for each aptamer-conjugate concentration were plotted, and the results are shown in Figure 4.13.

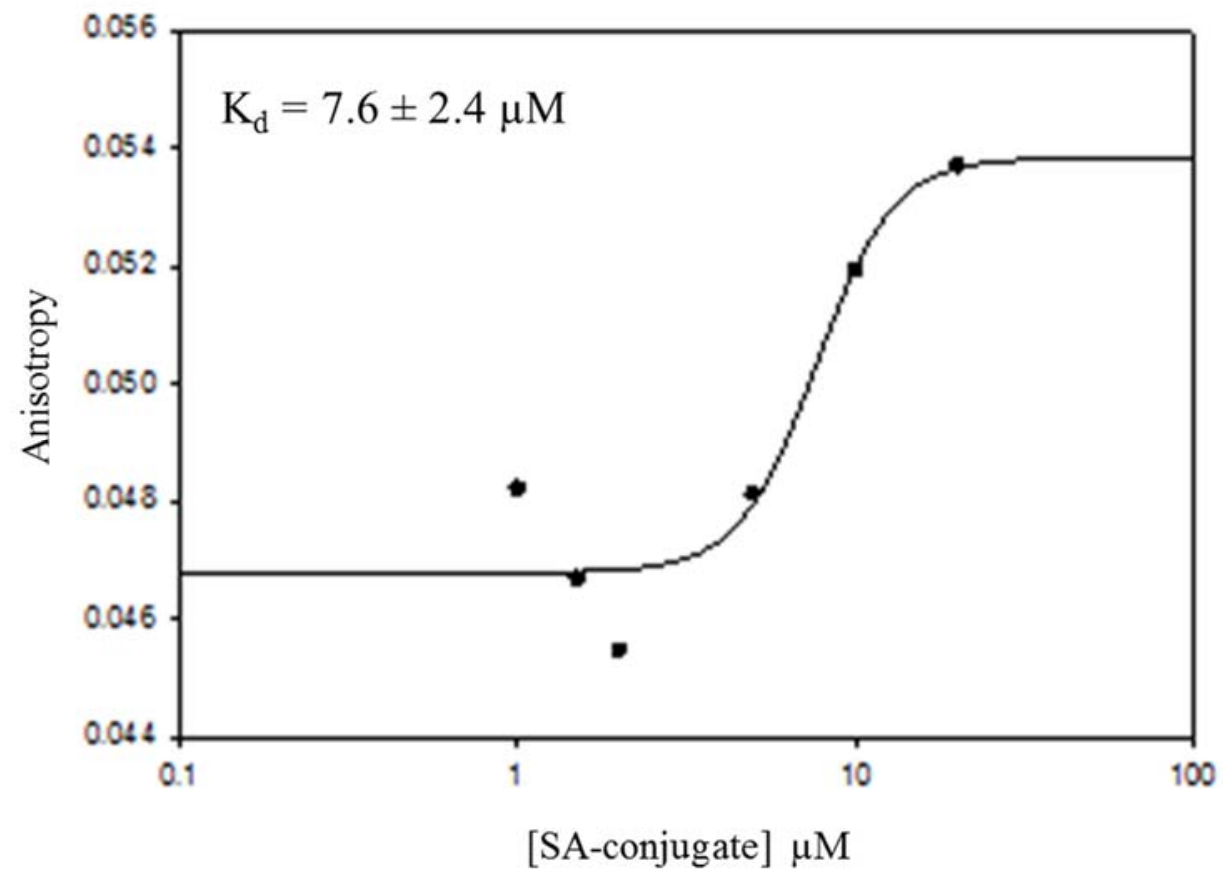

Figure 4.13: The apparent dissociation constant of SA-conjugates binding sulforhodamine $B$ was determined using fluorescence anisotropy. A range of SA-conjugate concentrations was incubated with $500 \mathrm{nM}$ SB for one hour prior to anisotropy measurements. Anisotropy values were measured with excitation and emission wavelengths of $556 \mathrm{~nm}$ and $582 \mathrm{~nm}$, respectively. The average of six replicates was plotted using SigmaPlot software, and fit using the Standard Curves Analysis feature. An apparent $K_{d}$ of $7.6 \pm 2.4 \mu \mathrm{M}$ was determined, with error from the curve fitting.

Using fluorescence anisotropy, an apparent dissociation constant of $7.6 \pm 2.4 \mu \mathrm{M}$ was obtained for the SA-conjugate binding SB in solution. The sulforhodamine B aptamer was reported to bind $\mathrm{SB}$ in solution with a $\mathrm{K}_{\mathrm{d}}$ around $660 \pm 60 \mathrm{nM}$, suggesting that while the aptamer-conjugate was still able to bind its target, the addition of the hydrophobic tail lowered 
the aptamer's affinity by about an order of magnitude. ${ }^{248}$ It has been shown that the sulforhodamine B aptamer forms a G-quadruplex structure that is necessary for target binding. ${ }^{248}$ It is possible that the addition of the 12-carbon hydrophobic chain to the aptamer could slightly hinder the aptamer's folding, causing a decrease in binding affinity. This change in affinity was also observed when the sulforhodamine B aptamer was incorporated into a PEM film, which could have also hindered the aptamer's folding. When embedded within the film, the aptamer was still able to bind $\mathrm{SB}$, however the apparent $\mathrm{K}_{\mathrm{d}}$ for the embedded aptamer was $16 \mu \mathrm{M}$ which was 20x higher than the $K_{d}$ for the free aptamer. ${ }^{247}$ The Chen group also reported an increased $K_{d}$ for SA embedded within PEM films, observing $\mathrm{K}_{\mathrm{dS}}$ around $6-9 \mu \mathrm{M}$, compared to the $660 \pm 60$ $\mathrm{nM}$ reported for SA in solution. ${ }^{248,251}$ Since the sulforhodamine B aptamer is known to form a Gquadruplex, it is also possible that bringing multiple aptamer strands in close proximity by incorporating them into nanostructures would allow these G-quadruplexes to interact. With these aptamers in close proximity, it is possible that intermolecular G-quadruplexes are formed that would have to be overcome in order to bind target molecules, which could hinder the affinity. To investigate whether the aptamer-conjugates could bind SB when they were incorporated into polymersomes, a range of SA- and RO-polymersomes were incubated with SB for an hour, and examined using fluorescence anisotropy, with the results shown in Figure 4.14. 


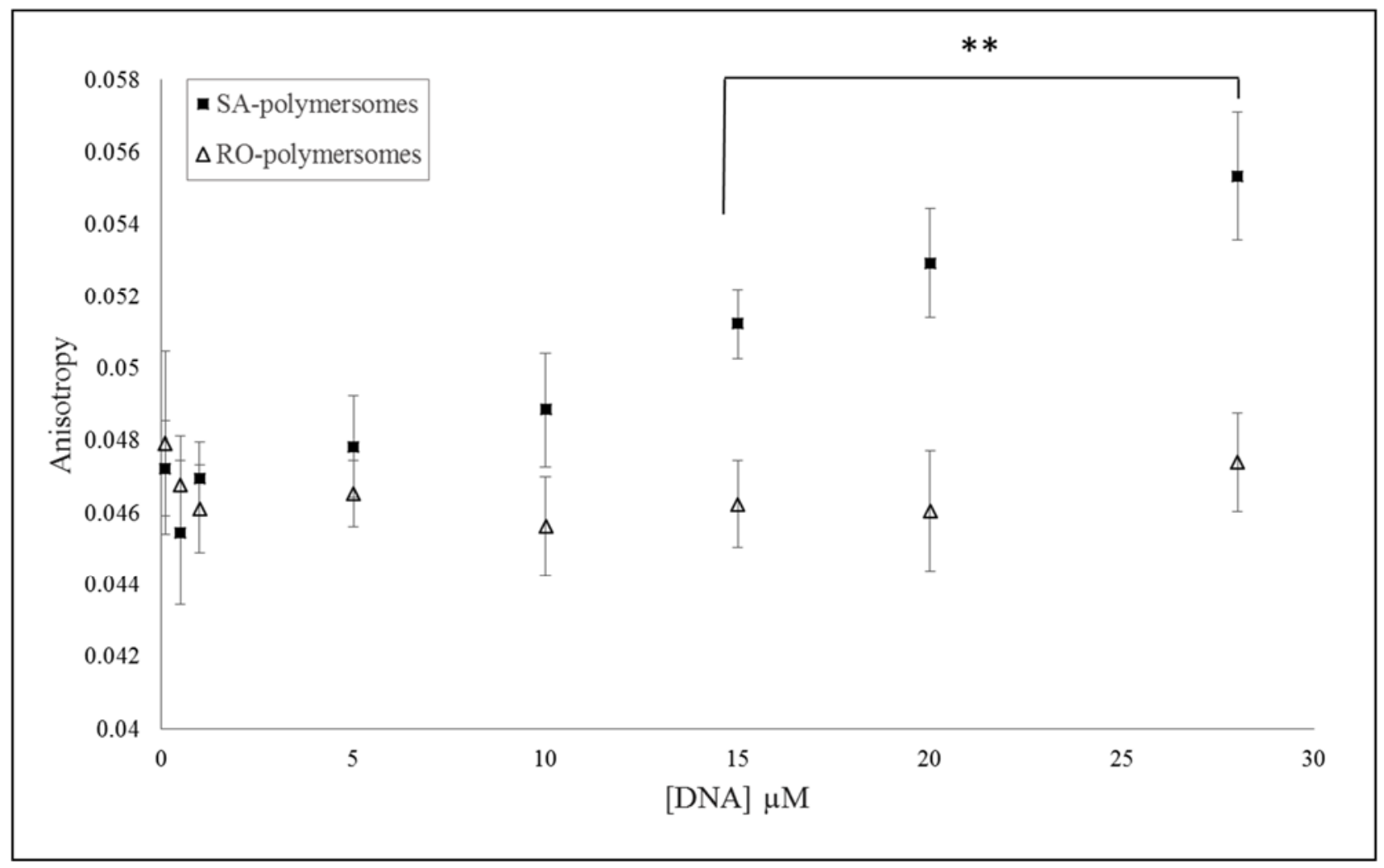

Figure 4.14: The binding of aptamer-polymersomes to sulforhodamine B was studied using fluorescence anisotropy. A range of aptamer-polymersome concentrations were incubated with $500 \mathrm{nM}$ SB for one hour. Anisotropy values were measured with excitation and emission wavelengths of $556 \mathrm{~nm}$ and $582 \mathrm{~nm}$, respectively. Error bars represent the standard deviation of six replicates. A one-way ANOVA analysis determined no statistical difference between the anisotropy values obtained for the RO-polymersomes at all concentrations. A significant difference was found for values obtained for SApolymersomes from 15-28 $\mu \mathrm{M}$, compared to the highest concentration of RO-polymersomes $(\mathbf{P}<\mathbf{0 . 0 1})$.

Figure 4.14 shows that an increase in anisotropy values was obtained as the concentration of SA-polymersomes incubated with SB was increased, which indicated more SB was being bound with increasing SA-polymersome concentration. Statistical analysis (one-way ANOVA) showed that there was no difference between the anisotropy values obtained for ROpolymersomes at all concentrations tested, showing that these RO-polymersomes did not bind SB in solution. A significant difference $(\mathrm{P}<0.01)$ was observed between the anisotropy values obtained for SA-polymersomes from 15-28 $\mu \mathrm{M}$ when compared to the highest concentration of RO-polymersome, which indicated that the SA-polymersomes were able to bind SB in solution. 
The ability of aptamers to bind their targets when they are incorporated into these polymersomes makes these structures promising vehicles for targeted-delivery applications.

\subsubsection{Effect of target-binding on aptamer-polymersome morphology}

Section 4.5.5.4 showed that the sulforhodamine B aptamer was able to bind its target dye when conjugated to a 12-carbon hydrophobic chain, as well as when it was incorporated into SApolymersomes. To investigate these aptamer-polymersomes as possible targeted-delivery vehicles, it was of interest to examine any effect target-binding had on the polymersome morphology. If target-binding destabilized the polymersome, any encapsulated cargo would be released in a one-time burst upon aptamer-target binding. If the polymersome was not destabilized by target-binding, a more prolonged release of cargo could be achieved. To examine the morphology of the SA-polymersomes after exposure to the target, the SA-polymersomes were incubated with SB for 30 minutes, deposited on a TEM grid, and dried for 2 hours before imaging. The resulting morphology is shown in Figure 4.15. 

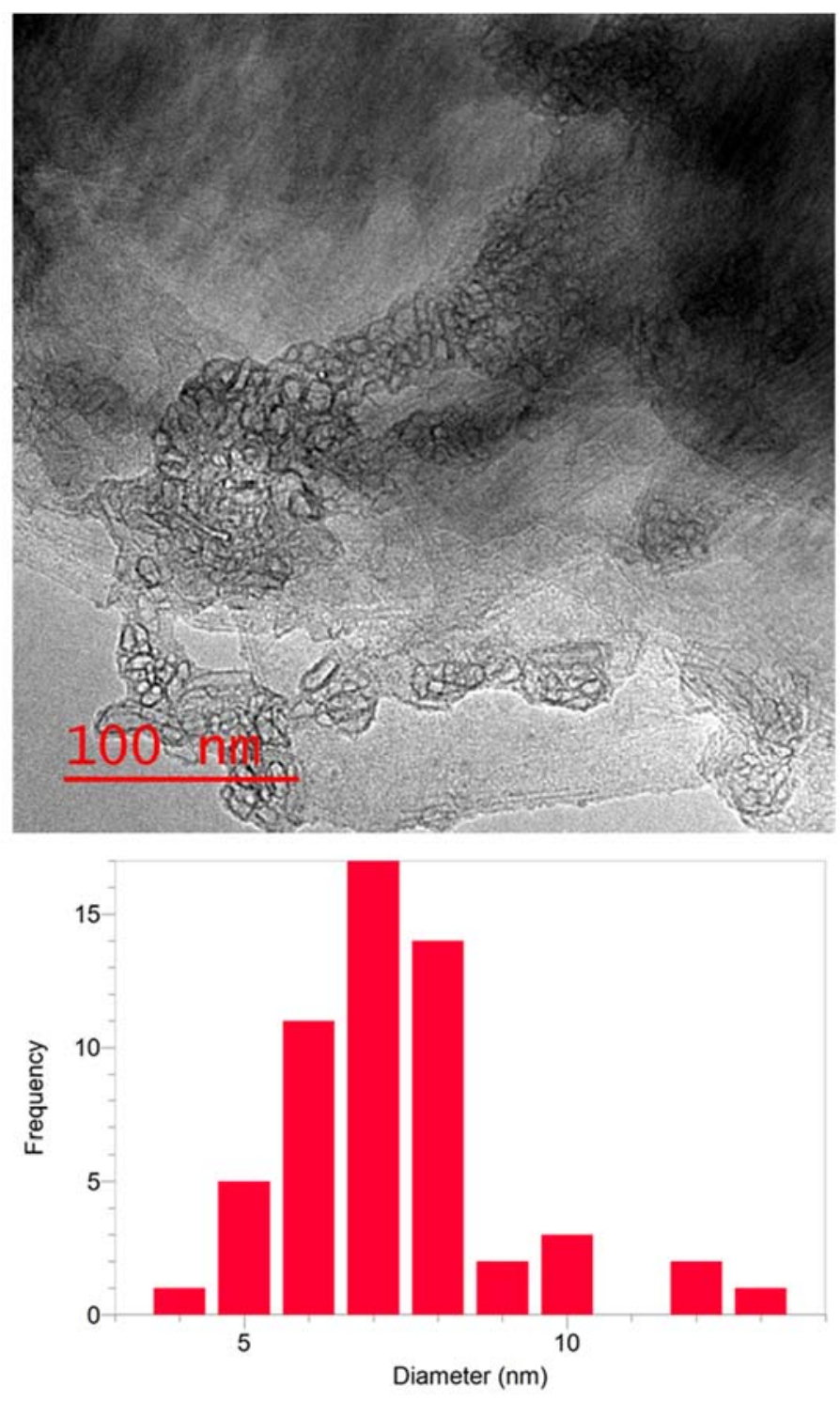

Average $=7.3 \pm 1.7 \mathrm{~nm}$

Figure 4.15: The effect of target-binding on aptamer-polymersome morphology was examined using TEM (Top image). SA-polymersomes $(40 \mu M)$ were incubated with SB (80 $\mu M)$ for 30 minutes prior to preparing the sample for TEM. The SA-polymersomes were measured using AmScope Toupview Camera software (bottom image).

Figure 4.15 shows that the small polymersome morphology of SA-polymersomes was maintained even after incubation with SB, with an average diameter of $7.3 \pm 1.7 \mathrm{~nm}$. This diameter falls within the standard deviation of the diameter observed for SA-polymersomes without target present (shown in Figure 4.6), indicating that incubation with the target did not 
cause immediate rupture of the SA-polymersomes. Rather than a burst-release of cargo, this

finding suggested that these aptamer-polymersomes could potentially be used for targeting and a more prolonged release of a cargo. Further studies are needed to investigate how the SApolymersome morphology changes over time with and without target molecules present, as well as with an encapsulated cargo molecule. Before the exudate-binding aptamers can be incorporated into this aptamer-polymersome system, the stability of the polymersomes in more complex media, such as soil solution should also be studied.

\subsubsection{Conclusions and Future Directions}

In this chapter, the sulforhodamine B aptamer was successfully incorporated into amphiphilic conjugates, which self-assembled into nanoscale polymersome structures. Altering the hydrophobic tail from 10 to 16 carbons in length did not affect the aptamer-polymersome morphology or stability. Aptamer-polymersomes synthesized from all chain-lengths remained stable in water over a nine day period. The aptamer-conjugates were able to bind their target with only a slight reduction in binding affinity and when aptamer-conjugates were incorporated into polymersomes, they maintained this target-binding ability. These aptamer-polymersomes have the stability and target-recognition abilities to make them promising targeted-delivery vehicles. Future work to investigate the stability of these aptamer-polymersomes in complex matrices, as well as cargo loading and release studies would help move these aptamer-polymersomes into useful applications. 
5 Future directions and contributions to knowledge 
The development of aptamer materials and delivery systems has often been focused on medically relevant targets, but in this work, challenges facing agriculture are discussed that aptamers could have a role in solving. The research described in this thesis shows the potential that aptamers have to bind to important exudate targets (Chapters 2 and 3), and to be incorporated into sensing platforms (Chapter 3 ) as well as targeted delivery vehicles and smart materials (Chapter 4). The selection of exudate-binding aptamers and the design of new materials could help alleviate the environmental impacts of fertilizer and pesticide use.

Chapter 2 of this work describes the selection of novel exudate-binding aptamers for the target L-serine. The most promising aptamer was optimized to reduce its cost of production, and to increase its resistance to nucleases (Chapter 3). This aptamer was also incorporated into a colorimetric AuNP test (Chapter 3), which could be optimized and incorporated into a lateral flow device, for example. ${ }^{325}$ The use of the selected L-serine-binding aptamers to create fast and inexpensive colorimetric tests for L-serine detection could provide farmers with information to improve the timing of fertilizer applications. The incorporation of the L-serine aptamer into biodegradable materials could allow for the design of smart fertilizer products, increasing the fertilizer use efficiency, and reducing the amount of nitrogen and phosphorus leaching into the environment. Aptamers that already exist for exudate targets (Chapter 1) could be used directly in simple, colorimetric sensors that could help inform on fertilizer or pesticide application. For example, the glutamate aptamer could be incorporated into a sensor that would indicate iron deficiency in maize crops. ${ }^{138,139}$ Many exudate targets were discussed in chapter 1 for which novel selections could produce useful aptamers that could be incorporated into sensor and delivery applications. 
Performing SELEX for small molecule targets has often been plagued with difficult characterization methods and lengthy selection protocols. This work investigated selection strategies to improve the process for small molecule aptamer development, using three SELEX strategies, which each produced a small molecule-binding aptamer. Chapter 2 and 3 discussed the utility of redirecting an existing selection pool for similar targets to produce selective aptamers with high affinity. Many selections have been performed for small molecule targets, and this work could encourage researchers to make use of their existing SELEX pools to expedite the selection of new aptamers. ${ }^{24,256}$ Creating a SELEX pool from a randomized aptamer also produced target-binding aptamers, which could allow researchers to make use of published aptamers to expedite their new selections. ${ }^{18}$ These findings will contribute to the faster selection and application of novel aptamers.

The development of nanostructures in which aptamers could easily be incorporated would encourage the creation of novel smart materials and targeted delivery devices. In this work, an existing aptamer was incorporated into aptamer-polymersomes by the simple addition of a hydrophobic chain (Chapter 4). This system was shown to create self-assembling nanostructures of consistent morphology that were able to bind their target. This system could be directly applied to existing aptamers to create targeted delivery vehicles for use in both medical and agricultural applications. Further work to investigate the effect of target-binding on aptamerpolymersome stability, as well as cargo encapsulation and release studies would be required. Examining the stability of these aptamer-polymersomes in complex matrices such as blood serum and soil could also expedite their use in novel applications.

The work described in this thesis contributes to the improvement of small molecule aptamer selections, and to the incorporation of aptamers into useful sensing and delivery 
applications. The development of exudate-binding aptamers and their incorporation into smart materials could provide novel solutions to agricultural problems 


\section{References}

(1) Alsaafin, A.; McKeague, M. Biosens. Bioelectron. 2017, 94 (December 2016), 94-106.

(2) Jenison, R. D.; Gill, S. C.; Pardi, A.; Polisky, B. Science (80-. ). 1994, 263 (5152), 14251429.

(3) Keefe, A. D.; Pai, S.; Ellington, A. Nat. Rev. Drug Discov. 2010, 9 (7), 537-550.

(4) Cruz-Toledo, J.; McKeague, M.; Zhang, X.; Giamberardino, A.; McConnell, E.; Francis, T.; DeRosa, M. C.; Dumontier, M. Database 2012, 2012, 1-8.

(5) Nguyen, V. T.; Kwon, Y. S.; Gu, M. B. Curr. Opin. Biotechnol. 2017, 45, 15-23.

(6) Zainol Abidin, A.; Rahim, R.; Md Arshad, M.; Fatin Nabilah, M.; Voon, C.; Tang, T.; Citartan, M. Sensors 2017, 17 (6), 1180.

(7) Poolsup, S.; Kim, C.-Y. Curr. Opin. Biotechnol. 2017, 48, 180-186.

(8) Chen, K.; Liu, B.; Yu, B.; Zhong, W.; Lu, Y.; Zhang, J.; Liao, J.; Liu, J.; Pu, Y.; Qiu, L.; Zhang, L.; Liu, H.; Tan, W. Wiley Interdiscip. Rev. Nanomedicine Nanobiotechnology 2017, $9(3), 1-15$.

(9) Mastronardi, E.; DeRosa, M. C. In Industrial Applications for Intelligent Polymers and Coatings; Springer International Publishing: Cham, 2016; pp 185-203.

(10) Mayer, G. Angew. Chemie Int. Ed. 2009, 48 (15), 2672-2689.

(11) Schmidt, K. S.; Borkowski, S.; Kurreck, J.; Stephens, A. W.; Bald, R.; Hecht, M.; Friebe, M.; Dinkelborg, L.; Erdmann, V. A. Nucleic Acids Res. 2004, 32 (19), 5757-5765.

(12) Tolle, F.; Mayer, G. Chem. Sci. 2013, 4 (1), 60-67.

(13) Bruno, J. G. Pharmaceuticals 2013, 6 (3), 340-357.

(14) Ellington, A. D.; Szostak, J. W. Nature 1990, 346, 818-822.

(15) Tuerk, C.; Gold, L. Science (80-. ). 1990, 249 (4968), 505-510.

(16) Robertson, D. L.; Joyce, G. F. Nature 1990, 344, 467-468.

(17) Stoltenburg, R.; Reinemann, C.; Strehlitz, B. Biomol. Eng. 2007, 24 (4), 381-403.

(18) McKeague, M.; McConnell, E. M.; Cruz-Toledo, J.; Bernard, E. D.; Pach, A.; Mastronardi, E.; Zhang, X.; Beking, M.; Francis, T.; Giamberardino, A.; Cabecinha, A.; Ruscito, A.; Aranda-Rodriguez, R.; Dumontier, M.; DeRosa, M. C. J. Mol. Evol. 2015, 81 (5-6), 150-161.

(19) Shangguan, D.; Li, Y.; Tang, Z.; Cao, Z. C.; Chen, H. W.; Mallikaratchy, P.; Sefah, K.; Yang, C. J.; Tan, W. Proc. Natl. Acad. Sci. U. S. A. 2006, 103 (32), 11838-11843. 
(20) Shangguan, D.; Bing, T.; Zhang, N. In Aptamers Selected by Cell-SELEX for Theranostics; Tan, W., Fang, X., Eds.; Springer, 2015; pp 13-33.

(21) Chen, F.; Zhou, J.; Luo, F.; Mohammed, A. B.; Zhang, X. L. Biochem. Biophys. Res. Commun. 2007, 357 (3), 743-748.

(22) Hamula, C.; Zhang, H.; Guan, L. L.; Li, X.-F.; Le, X. C. Anal. Chem. 2008, 80, 78127819.

(23) Tang, Z.; Parekh, P.; Turner, P.; Moyer, R. W.; Tan, W. Clin. Chem. 2009, 55 (4), 813822.

(24) McKeague, M.; DeRosa, M. C. J. Nucleic Acids 2012, 2012, 1-20.

(25) Ruff, K. M.; Snyder, T. M.; Liu, D. R. J. Am. Chem. Soc. 2010, 132 (27), 9453-9464.

(26) Luo, X.; McKeague, M.; Pitre, S.; Dumontier, M.; Green, J.; Golshani, A.; Derosa, M. C.; Dehne, F. RNA 2010, 16 (11), 2252-2262.

(27) Lipi, F.; Chen, S.; Chakravarthy, M.; Rakesh, S.; Veedu, R. N. RNA Biol. 2016, 13 (12), 1232-1245.

(28) Darmostuk, M.; Rimpelova, S.; Gbelcova, H.; Ruml, T. Biotechnol. Adv. 2014, 33 (6), 1141-1161.

(29) Quang, N. N.; Perret, G.; Ducongé, F. Pharmaceuticals 2016, 9 (4), 1-15.

(30) Ruigrok, V. J. B.; Levisson, M.; Eppink, M. H. M.; Smidt, H.; van der Oost, J. Biochem. J. 2011, $436(1), 1-13$.

(31) Menger, M.; Yarman, A.; Erdőssy, J.; Yildiz, H.; Gyurcsányi, R.; Scheller, F. Biosensors 2016, 6 (3), 35.

(32) Roy, I.; Gupta, M. N. Chem. Biol. 2003, 10, 1161-1171.

(33) Pardo, R.; Zayat, M.; Levy, D. Chem. Soc. Rev. 2011, 40 (2), 672-687.

(34) Seeboth, A.; Ruhmann, R.; Mühling, O. Materials (Basel). 2010, 3 (12), 5143-5168.

(35) Mortimer, R. J. Annu. Rev. Mater. Sci. 2011, 41, 241-268.

(36) Scherer, M. R. J.; Steiner, U. Nano Lett. 2013, 13 (7), 3005-3010.

(37) Seeboth, A.; Lötzsch, D.; Ruhmann, R.; Muehling, O. Chem. Rev. 2014, 114 (5), $3037-$ 3068 .

(38) Kline, W. M.; Lorenzini, G.; Sotzing, G. A. 2014, 73-80.

(39) Zhang, J.; Zou, Q.; Tian, H. Adv. Mater. 2013, 25 (3), 378-399.

(40) Sun, L.; Huang, W. M.; Ding, Z.; Zhao, Y.; Wang, C. C.; Purnawali, H.; Tang, C. Mater. Des. 2012, 33 (1), 577-640. 
(41) Yu, Z.; Zhang, Q.; Li, L.; Chen, Q.; Niu, X.; Liu, J.; Pei, Q. Adv. Mater. 2011, 23 (5), 664-668.

(42) Iliuk, A. B.; Hu, L.; Tao, W. A. Anal. Chem. 2011, 83 (12), 4440-4452.

(43) Cibiel, A.; Dupont, D. M.; Ducongé, F. Pharmaceuticals 2011, 4 (9), 1216-1235.

(44) Duan, N.; Wu, S.; Chen, X.; Huang, Y.; Xia, Y.; Ma, X.; Wang, Z. J. Agric. Food Chem. 2013, 61 (13), 3229-3234.

(45) Xiang, D.; Shigdar, S.; Qiao, G.; Wang, T.; Kouzani, A. Z.; Zhou, S. Theranostics 2015, 5 (1).

(46) Mastronardi, E.; Foster, A.; Zhang, X.; DeRosa, M. C. Sensors (Basel). 2014, 14 (2), 3156-3171.

(47) Yoshida, R.; Okano, T. Biomed. Appl. Hydrogels Handb. 2010, 19-43.

(48) Caló, E.; Khutoryanskiy, V. V. Eur. Polym. J. 2014, 65, 252-267.

(49) Yang, H.; Liu, H.; Kang, H.; Tan, W. J. Am. Chem. Soc. 2008, 130 (20), 6320-6321.

(50) Wei, B.; Cheng, I.; Luo, K. Q.; Mi, Y. Angew. Chemie - Int. Ed. 2008, 47 (2), 331-333.

(51) El-Hamed, F.; Dave, N.; Liu, J. Nanotechnology 2011, 22 (49), 494011.

(52) Soontornworajit, B.; Zhou, J.; Wang, Y. Soft Matter 2010, 6 (17), 4255.

(53) Battig, M. R.; Soontornworajit, B.; Wang, Y. J. Am. Chem. Soc. 2012, 134 (30), 1241012413.

(54) He, X.; Wei, B.; Mi, Y. Chem. Commun. (Camb). 2010, 46 (34), 6308-6310.

(55) Yoshida, W.; Yokobayashi, Y. Chem. Commun. (Camb). 2007, 9 (2), 195-197.

(56) Liu, Y.; Ren, J.; Qin, Y.; Li, J.; Liu, J.; Wang, E. Chem. Commun. 2012, 48 (6), 802.

(57) Xu, X.; Zhang, J.; Yang, F.; Yang, X. Chem. Commun. (Camb). 2011, 47 (33), 94359437.

(58) You, M.; Zhu, G.; Chen, T.; Donovan, M. J.; Tan, W. 2015.

(59) Zhu, C.-L.; Song, X.-Y.; Zhou, W.-H.; Yang, H.-H.; Wen, Y.-H.; Wang, X.-R. J. Mater. Chem. 2009, 19 (41), 7765.

(60) Wang, J.; Lu, J.; Su, S.; Gao, J.; Huang, Q.; Wang, L.; Huang, W.; Zuo, X. Biosens. Bioelectron. 2015, 65, 171-175.

(61) Yin, B.-C.; Ye, B.-C.; Wang, H.; Zhu, Z.; Tan, W. Chem. Commun. 2012, 48 (9), 1248.

(62) Jiang, Y.; Liu, N.; Guo, W.; Xia, F.; Jiang, L. J. Am. Chem. Soc. 2012, 134 (37), 1539515401. 
(63) Zhu, X.; Zhang, B.; Ye, Z.; Shi, H.; Shen, Y.; Li, G. Chem. Commun. 2015, 51 (4), 640643.

(64) Schäfer, T.; Özalp, V. C. Chem. Commun. (Camb). 2015, 51 (25), 5471-5474.

(65) Wang, R.; Xu, L.; Li, Y. Biosens. Bioelectron. 2015, 67, 400-407.

(66) Zhu, C. L.; Lu, C. H.; Song, X. Y.; Yang, H. H.; Wang, X. R. J. Am. Chem. Soc. 2011, $133(5), 1278-1281$.

(67) Douglas, S. M.; Bachelet, I.; Church, G. M. Science (80-. ). 2012, 335, 831-834.

(68) Bachelet, I.; Church, G.; Douglas, S. DNA origami devices. WO2012061719 A3, 2012.

(69) Amir, Y.; Ben-Ishay, E.; Levner, D.; Ittah, S.; Abu-Horowitz, A.; Bachelet, I. Nat. Nanotechnol. 2014, 9 (5), 353-357.

(70) Derosa, M. C.; Monreal, C.; Schnitzer, M.; Walsh, R.; Sultan, Y. Nat. Nanotechnol. 2010, $5(2), 91$.

(71) Raun, W. R.; Johnson, G. V. Agron. J. 1999, 91 (3), 357.

(72) Fleischer, A.; O’Neill, M. A.; Ehwald, R. Plant Physiol. 1999, 121 (3), 829-838.

(73) Carpita, N.; Sabularse, D.; Montezinos, D.; Delmer, D. P. Science (80-. ). 1979, 205 (4411), 1144-1147.

(74) Eichert, T.; Goldbach, H. E. Physiol. Plant. 2008, 132 (4), 491-502.

(75) Rico, C. M.; Majumdar, S.; Duarte-Gardea, M.; Peralta-Videa, J. R.; Gardea-Torresdey, J. L. J. Agric. Food Chem. 2011, 59 (8), 3485-3498.

(76) Eichert, T.; Kurtz, A.; Steiner, U.; Goldbach, H. E. Physiol. Plant. 2008, 134 (1), 151160.

(77) Zambryski, P. J. Cell Biol. 2004, 164 (2), 165-168.

(78) Liu, Q.; Chen, B.; Wang, Q.; Shi, X.; Xiao, Z.; Lin, J.; Fang, X. Nano Lett. 2009, 9 (3), 1007-1010.

(79) Torney, F.; Trewyn, B. G.; Lin, V. S.-Y.; Wang, K. Nat. Nanotechnol. 2007, 2 (5), 295300.

(80) Ruttkay-Nedecky, B.; Krystofova, O.; Nejdl, L.; Adam, V. J. Nanobiotechnology 2017, 15 (1), 33 .

(81) Bhabra, G.; Sood, A.; Fisher, B.; Cartwright, L.; Saunders, M.; Evans, W. H.; Surprenant, A.; Lopez-Castejon, G.; Mann, S.; Davis, S. A.; Hails, L. A.; Ingham, E.; Verkade, P.; Lane, J.; Heesom, K.; Newson, R.; Case, C. P. Nat. Nanotechnol. 2009, 4 (12), 876-883.

(82) Foster, A.; DeRosa, M. C. Polymers (Basel). 2014, 6 (5), 1631-1654. 
(83) Mastronardi, E.; Tsae, P.; Zhang, X.; Monreal, C.; DeRosa, M. C. In Nanotechnologies in Food and Agriculture; Springer International Publishing: Cham, 2015; pp 25-67.

(84) Prasad, T. N. V. K. V.; Sudhakar, P.; Sreenivasulu, Y.; Latha, P.; Munaswamy, V.; Reddy, K. R.; Sreeprasad, T. S.; Sajanlal, P. R.; Pradeep, T. J. Plant Nutr. 2012, 35 (6), 905-927.

(85) Wan, Z. Nanosized urea and its production process. CN 1485303, 2004.

(86) Zhao, L.; Sun, Y.; Hernandez-Viezcas, J. A.; Servin, A. D.; Hong, J.; Niu, G.; PeraltaVidea, J. R.; Duarte-Gardea, M.; Gardea-Torresdey, J. L. J. Agric. Food Chem. 2013, 61 (49), 11945-11951.

(87) El-Kereti, M. A.; El-feky, S. A.; Khater, M. S.; Osman, Y. A.; El-sherbini, E. A. Recent Pat. Food. Nutr. Agric. 2013, 5 (3), 169-181.

(88) Pandey, A. C.; S. Sanjay, S.; S. Yadav, R. J. Exp. Nanosci. 2010, 5 (6), 488-497.

(89) Wei, L.; Li, J.; Zhu, C. Nano-selenium amino acid foliar fertilizer and preparation method of the same. CN 102391053, 2012.

(90) Khodakovskaya, M.; Dervishi, E.; Mahmood, M.; Xu, Y.; Li, Z.; Watanabe, F.; Biris, A. S. ACS Nano 2009, 3 (10), 3221-3227.

(91) Biris, A. S.; Khodakovskaya, M. Method of using carbon nanotubes to affect seed germination and plant growth. WO 2011059507, 2011.

(92) Tripathi, S.; Sonkar, S. K.; Sarkar, S. Nanoscale 2011, 3 (3), 1176.

(93) Mondal, A.; Basu, R.; Das, S.; Nandy, P. J. Nanoparticle Res. 2011, 13 (10), 4519-4528.

(94) Park, M.; Kim, C. Y.; Lee, D. H.; Choi, C. L.; Choi, J.; Lee, S.-R.; Choy, J.-H. J. Phys. Chem. Solids 2004, 65 (2-3), 409-412.

(95) Yu, E. Q. Nano diatomite and zeolite ceramic crystal powder for use as a plant growth medium. US 6942714, 2005.

(96) Y., G. Long-acting added-acting additive for composite fertilizer. CN 1919804A, 2007.

(97) Wu, C.; Wu, Q. Nitrogen fertilizer-specific functional slow-release agent. CN 101628838, 2010 .

(98) Gai, G.; Zhao, F.; Liu, C.; Yang, Y. Granular fertilizer coated with mineral micro/nanopowder. CN 201883046, 2011.

(99) Cao, F.; Mou, S.; Li, M. Palygorskite material-based sustained-release nitrogen fertilizer. CN 1978396, 2007.

(100) Cao, F.; Mou, S.; Li, M. Palygorskite material-based sustained-release composite fertilizer. CN 1978399, 2007. 
(101) Cao, F.; Mou, S.; Li, M. Palygorskite material-based sustained-release potash fertilizer. CN 10127832, 2007.

(102) Cao, F.; Mou, S.; Li, M. Palygorskite material-based sustained-release phosphorus fertilizer. CN 1978397, 2007.

(103) Zhang, F.; Wang, Y.; Zhang, J.; Yao, Q.; Liu, X.; Xiao, Q. Preparation of nano-micro paraffin wax/kaolin mixture for film coating agent. CN 1635027, 2005.

(104) Price, R. R.; Wagner, A. A method for treating agricultural crops using materials associated with tubular carriers. US 2008194406, 2008.

(105) NaturalNano Corp. natural nano http://naturalnano.com/ (accessed Jul 19, 2017).

(106) Nagarsekar, K.; Ashtikar, M.; Steiniger, F.; Thamm, J.; Schacher, F.; Fahr, A. Soft Matter 2016, 12 (16), 3797-3809.

(107) Yavitz, E. Q. Plant protection, feeding, and watering by foliar application of nanoscale particles. US 2006014645, 2006.

(108) Wei, Z.; Wang, Y.; He, C.; Wei, W.; Cui, J. Phosphorus fertilizer comprising nanohydroxyapatite and its preparation process. CN 101973791, 2011.

(109) Kottegoda, N.; Munaweera, I.; Madusanka, A. N.; Karunaratne, V. Compositions for sustained release of agricultural macronutrients and process thereof. WO 2011151724 , 2011.

(110) Kottegoda N, Munaweera I, Samaranayake L, Gunasekara S, De Alwis A, K. V; AN, M. Cellulose based sustained release macronutrient composition for fertilizer application. US 20110296887, 2011.

(111) Kottegoda N, Priyadharshana G, Sandaruwan C, Dahanayake D, Gunasekara S, A. A.; V, K. Composition and method for sustained release of agricultural macronutrients. US 20130098125, 2013.

(112) Kottegoda N, Munaweera I, Madusanka N, K. V. Curr. Sci. 2011, 101, 73-78.

(113) Giroto, A. S.; Fidélis, S. C.; Ribeiro, C. RSC Adv. 2015, 5 (126), 104179-104186.

(114) Kottegoda, N.; Sandaruwan, C.; Priyadarshana, G.; Siriwardhana, A.; Rathnayake, U. A.; Berugoda Arachchige, D. M.; Kumarasinghe, A. R.; Dahanayake, D.; Karunaratne, V.; Amaratunga, G. A. J. ACS Nano 2017, 11 (2), 1214-1221.

(115) Zhang F, Shi C, Z. B. Production technique of coating cement for nanosulfonated lignin mixture fertilizer. CN 1417173A, 2003.

(116) $\mathrm{Du}, \mathrm{Y}$. Method for manufacturing fertilizer cementing agent by using paper-making black liquor. CN 101081795A, 2007.

(117) Zhang F, Shi C, Wang Y, Zhang J, W. R. Production of sustained/controlled release fertilizer used for greenhouse tomato. CN 1654445, 2005. 
(118) Zhang F, Yao Q, Wang Y, Z. J. Preparation method of cementing and film-forming agent for fertilizer from nanoparticle-submicron particles of mixed polymer of polyvinyl alcohol. CN 1609078, 2005.

(119) Zhang F, Yao C, Wang Y, Zhang J, Liu X, Xiao Q, F. Z. Preparation of polymeric mixture from waste polystyrene foam and useful for fertilizer adhesive or capsules. CN 1631952 , 2005.

(120) Zhang, F. Manufacture of nano olefin-starch blend as fertilizer packaging film or granulating binder. CN 1546543, 2004.

(121) Lin, C. Novel sustained-release nanosized fertilizer and production method therefor. CN $101225010,2008$.

(122) Li F, Pham H, A. D. Methods to produce polymer nanoparticles and formulations of agricultural active ingredients. WO 2010035118, 2010.

(123) Yuvaraj, M.; Subramanian, K. S. Soil Sci. Plant Nutr. 2014, 61 (2), 319-326.

(124) Barati, A. Nanocomposite superabsorbent containing fertilizer nutrients used in agriculture. US 20100139347, 2010.

(125) Cai, D. Controlled release of low-cost environment friendly nitrogenous fertilizer. CN 101041606, 2007.

(126) Zhang F, Zhao B, Zhang J, Shi C, He X, Zhang J, W. R. Technical process for producing clay nanoparticle-polyester blended polymer as binder for encapsulating fertilizer. $\mathrm{CN}$ 1414033, 2003.

(127) Dong J, He A, Han Z, W. L. Polyolefin/alkyltriphenylphosphonium halide-modified montmorillonite nanocomposite, its preparation and application. CN 1789318, 2006.

(128) Liu, X.; Feng, Z.; Zhang, F.; Zhang, S.; He, X. Agric. Sci. China 2006, 5 (9), 700-706.

(129) Zhang F, Liu X, Xiao Q, Wang Y, Z. J. Nanoscience 2006, No. 1, 90-95.

(130) Zhang F, Wang R, Xiao Q, Wang Y, Z. J. Nanoscience 2006, No. 11, 18-26.

(131) Badri, D. V.; Vivanco, J. M. Plant, Cell Environ. 2009, 32 (6), 666-681.

(132) Bais, H. P.; Weir, T. L.; Perry, L. G.; Gilroy, S.; Vivanco, J. M. Annu. Rev. Plant Biol. 2006, 57, 233-266.

(133) Akiyama, K.; Matsuzaki, K.; Hayashi, H. Nature 2005, 435 (7043), 824-827.

(134) Jose, S.; Gillespie, A. Plant Soil 1998, 203 (2), 191-197.

(135) Haichar, F. el Z.; Santaella, C.; Heulin, T.; Achouak, W. Soil Biol. Biochem. 2014, 77, 6980 .

(136) Rugova, A.; Puschenreiter, M.; Koellensperger, G.; Hann, S. Anal. Chim. Acta 2017, 956, 
$1-13$.

(137) van Dam, N. M.; Bouwmeester, H. J. Trends Plant Sci. 2016, 21 (3), 256-265.

(138) Carvalhais, L. C.; Dennis, P. G.; Fedoseyenko, D.; Hajirezaei, M.-R.; Borriss, R.; von Wirén, N. J. Plant Nutr. Soil Sci. 2011, 174 (1), 3-11.

(139) Ohsawa, K.; Kasamatsu, T.; Nagashima, J.-I.; Hanawa, K.; Kuwahara, M.; Ozaki, H.; Sawai, H. Anal. Sci. 2008, 24 (1), 167-172.

(140) Ouyang, R.; Lei, J.; Ju, H.; Xue, Y. Adv. Funct. Mater. 2007, 17 (16), 3223-3230.

(141) Yang, H. H.; Zhang, S. Q.; Tan, F.; Zhuang, Z. X.; Wang, X. R. J. Am. Chem. Soc. 2005, $127(5), 1378-1379$.

(142) Seong, H.; Lee, H.-B.; Park, K. J. Biomater. Sci. Polym. Ed. 2002, 13 (6), 637-649.

(143) Parmpi, P.; Kofinas, P. Biomaterials 2004, 25 (10), 1969-1973.

(144) Rostamizadeh, K.; Abdollahi, H.; Parsajoo, C. Int. Nano Lett. 2013, 3 (1), 20.

(145) Rajkumar, R.; Warsinke, A.; Möhwald, H.; Scheller, F. W.; Katterle, M. Talanta 2008, 76 (5), 1119-1123.

(146) Sreenath, K.; Prabhasankar, P.; Venkatesh, Y. P. Food Addit. Contam. 2006, 23 (9), 861869.

(147) Kraffczyk, I.; Trolldenier, G.; Beringer, H. Soil Biol. Biochem. 1984, 16 (4), 315-322.

(148) Fan, B.; Carvalhais, L. C.; Becker, A.; Fedoseyenko, D.; von Wirén, N.; Borriss, R. BMC Microbiol. 2012, 12, 116.

(149) Tarannum, N.; Singh, M. Am. J. Anal. Chem. 2011, 2 (8), 909-918.

(150) Prasad, B. B.; Srivastava, A.; Tiwari, M. P. Mater. Sci. Eng. C 2013, 33 (7), 4071-4080.

(151) Farhanah. Ab. Halim, N.; Noor. Ahmad, M.; Md. Shakaff, A. Y.; Deraman, N. Procedia Eng. 2013, 53, 64-70.

(152) Garcia, I. T. S.; Porto, F. G. D. S.; Do Amaral, Q. D. F.; Carreño, N. L. V; Martins, M. M.; Wallau, M. Surf. Interface Anal. 2008, 40 (5), 899-905.

(153) Ames, T. D.; Breaker, R. RNA Biol. 2011, 8 (1), 82-89.

(154) Chen, J.; Liang, R. P.; Wang, X. N.; Qiu, J. D. J. Chromatogr. A 2015, 1409, 268-276.

(155) Zhu, F.; Yan, X.; Liu, S. Anal. Methods 2015, 7 (20), 8740-8749.

(156) Zheng, X. F.; Lian, Q.; Wu, H. X.; Liu, H. M.; Song, S. T. Russ. J. Appl. Chem. 2015, 88 (1), 160-168.

(157) Zheng, X.-F.; Lian, Q.; Yang, H. RSC Adv. 2014, 4 (80), 42478-42485. 
(158) Mannironi, C.; Scerch, C.; Fruscoloni, P.; Tocchini-Valentini, G. P. RNA 2000, 6 (4), $520-527$.

(159) Legiewicz, M.; Yarus, M. J. Biol. Chem. 2005, 280 (20), 19815-19822.

(160) Majerfeld, I.; Yarus, M. RNA 1998, 4 (4), 471-478.

(161) Lozupone, C.; Changayil, S.; Majerfeld, I.; Yarus, M. RNA 2003, 9 (11), 1315-1322.

(162) A.N. Zyablov, A.V. Kalach, Y. Zhibrova, V.E. Selemenev, O. V. D. J. Anal. Chem. 2010, 65 (1), 2010.

(163) Hashemi-Moghaddam, H.; Toosi, M.; Toosi, M. Anal. Methods 2015, 0, 1-8.

(164) Mbukwa, E. A.; Msagati, T. A. M.; Mamba, B. B. Anal. Bioanal. Chem. 2013, 405 (12), 4253-4267.

(165) Famulok, M. J. Am. Chem. Soc. 1994, $116(5)$, 1698-1706.

(166) Harada, K.; Frankel, A. D. EMBO J. 1995, 14 (23), 5798-5811.

(167) Geiger, A.; Burgstaller, P.; Von der Eltz, H.; Roeder, A.; Famulok, M. Nucleic Acids Res. 1996, 24 (6), 1029-1036.

(168) Majerfeld, I.; Yarus, M. Nat. Struct. Biol. 1994, 1 (5), 287-292.

(169) Prasad, B. B.; Tiwari, K.; Singh, M.; Sharma, P. S.; Patel, A. K.; Srivastava, S. J. Sep. Sci. 2009, 32 (7), 1096-1105.

(170) Majerfeld, I.; Puthenvedu, D.; Yarus, M. J. Mol. Evol. 2005, 61 (2), 226-235.

(171) Najafizadeh, P.; Ebrahimi, S. A.; Panjehshahin, M. R.; Rezayat Sorkhabadi, S. M. Iran. J. Med. Sci. 2014, 39 (6), 552-558.

(172) Yang, L. F.; Hu, X. L.; Guan, P.; Li, J.; Wu, D. F.; Gao, B. J. Appl. Polym. Sci. 2015, 132 (36), 1-9.

(173) Illangasekare, M.; Yarus, M. J. Mol. Evol. 2002, 54 (3), 298-311.

(174) Zheng, X.; Lin, R.; Zhou, X.; Zhang, L.; Lin, W. Anal. Methods 2012, 4 (2), 482.

(175) Prasad, B. B.; Pandey, I. Sensors Actuators, B Chem. 2013, 181, 596-604.

(176) Park, H.-E.; Tian, M.; Row, K.-H. Sep. Sci. Technol. 2014, 49 (9), 1401-1406.

(177) Zhao, W.; Wei, C.; Xia, Y.; Tang, B.; Yuan, J. L. In 3rd International Conference on Bioinformatics and Biomedical Engineering; Beijing, 2009; pp 1-4.

(178) Jiang, Y.; Tong, A. J. J. Appl. Polym. Sci. 2004, 94 (2), 542-547.

(179) Booker, K.; Bowyer, M. C.; Holdsworth, C. I.; McCluskey, A. Chem. Commun. 2006, 1730-1732. 
(180) Long, Y.; Pfeiffer, F.; Mayer, G.; Schrøder, T. D.; Özalp, V. C.; Olsen, L. F. 2016; pp 319.

(181) Shekarchizadeh, H.; Ensafi, A. A.; Kadivar, M. Mater. Sci. Eng. C 2013, 33 (6), 35533561 .

(182) Scherrmann, M. C.; Boutboul, A.; Estramareix, B.; Hoffmann, A. S.; Lubineau, A. Carbohydr. Res. 2001, 334 (4), 295-307.

(183) Barbani, N.; Rosellini, E.; Donati, M.; Costantino, P.; Cristallini, C.; Ciardelli, G. Polym. Int. 2017, 66 (6), 900-907.

(184) Striegler, S. Bioseparation 2001, 10 (6), 307-314.

(185) Balieiro, A. L.; Santos, R. A.; Pereira, M. M.; Figueiredo, R. T.; Freitas, L. S.; Alsina, O. L. S. De; Lima, A. S.; Soares, C. M. F. Brazilian J. Chem. Eng. 2016, 33 (2), 361-372.

(186) Bird, G. W.; Roy, T. C. Vox Sang. 1980, 38 (3), 169-171.

(187) Shen, F.; Ren, X. Rsc Adv. 2014, 4 (25), 13123-13125.

(188) Sun, X.; Pan, Q.; Yuan, C.; Wang, Q.; Tang, X.-L.; Ding, K.; Zhou, X.; Zhang, X.-L. J. Am. Chem. Soc. 2016, 138 (36), 11680-11689.

(189) Pan, Q.; Wang, Q.; Sun, X.; Xia, X.; Wu, S.; Luo, F.; Zhang, X.-L. Mol. Ther. 2014, 22 (5), 940-951.

(190) Monreal, C. M.; Schnitzer, M. I. 2015, 173-194.

(191) Monreal, C. M.; Schnitzer, M. The chemistry and biochemistry of organic components in the soil solutions of wheat rhizospheres, 1st ed.; Copyright (C) 2013 Elsevier Inc., 2013; Vol. 121.

(192) Weiss, R.; Molinelli, A.; Jakusch, M.; Mizaikoff, B. Bioseparation 2001, 10 (6), 379-387.

(193) Gao, D.; Wang, D. D.; Zhang, Q.; Yang, F. Q.; Xia, Z. N.; Zhang, Q. H.; Yuan, C. S. J. Agric. Food Chem. 2017, 65 (6), 1158-1166.

(194) Zhu, H.; Wang, Y.; Yuan, Y.; Zeng, H. Anal. Methods 2011, 3 (2), 348-355.

(195) Song, X.; Li, J.; Wang, J.; Chen, L. Talanta 2009, 80 (2), 694-702.

(196) Yin, Y.; Yan, L.; Zhang, Z.; Wang, J.; Luo, N. J. Sep. Sci. 2016, 39 (8), 1480-1488.

(197) Liang, R.; Chen, L.; Qin, W. Sci. Rep. 2015, 5 (1), 12462.

(198) Ye, H.; Chen, X.; Feng, Z. Adsorpt. Sci. Technol. 2016, No. 58.

(199) Wu, X. L.; Yu, S. J.; Kang, K. R. Food Chem. 2015, 170, 354-359.

(200) Jie, Z.; Xiwen, H. Anal. Chim. Acta 1999, 381 (1), 85-91. 
(201) Mullett, W. M.; Dirie, M. F.; Lai, E. P. C.; Guo, H.; He, X. Anal. Chim. Acta 2000, 414 (1-2), 123-131.

(202) Liu, X.; Zhou, T.; Du, Z.; Wei, Z.; Zhang, J. Soft Matter 2011, 7 (5), 1986.

(203) Liu, L.; Cao, Y.; Ma, P.; Qiu, C.; Xu, W.; Liu, H.; Huang, W. Rsc Adv. 2014, 4 (2), 605616.

(204) Yang, X.; Bing, T.; Mei, H.; Fang, C.; Cao, Z.; Shangguan, D. Analyst 2011, 136 (3), $577-585$.

(205) Niu, D.; Zhou, Z.; Yang, W.; Li, Y.; Xia, L.; Jiang, B.; Xu, W.; Huang, W.; Zhu, T. J. Appl. Polym. Sci. 2013, 130 (4), 2859-2866.

(206) Spears, T. Ottawa Citizen. Ottawa October 20, 2015.

(207) Leivo, J.; Mäkelä, J.; Rosenberg, J.; Lamminmäki, U. Anal. Biochem. 2016, 492 (2016), 27-29.

(208) Takeda, K.; Uemura, K.; Kobayashi, T. Anal. Chim. Acta 2007, 591 (1), 40-48.

(209) Zhang, Y.; Li, Y.; Hu, Y.; Li, G.; Chen, Y. J. Chromatogr. A 2010, 1217 (47), 7337-7344.

(210) Kugimiya, A.; Takeuchi, T. Electroanalysis 1999, 11 (15), 1158-1160.

(211) Qi, C.; Bing, T.; Mei, H.; Yang, X.; Liu, X.; Shangguan, D. Biosens. Bioelectron. 2013, $41,157-162$.

(212) Kirsch, N.; Alexander, C.; Lübke, M.; Whitcombe, M. ; Vulfson, E. . Polymer (Guildf). 2000, 41 (15), 5583-5590.

(213) Slavkovic, S.; Altunisik, M.; Reinstein, O.; Johnson, P. E. Bioorganic Med. Chem. 2015, 23 (10), 2593-2597.

(214) Parisi, O. I.; Morelli, C.; Puoci, F.; Saturnino, C.; Caruso, a; Sisci, D.; Trombino, G. E.; Picci, N.; Sinicropi, M. S. J. Mater. Chem. B 2014, 20 (38), 6619-6625.

(215) Gryshchenko, A. O.; Bottaro, C. S. Int. J. Mol. Sci. 2014, 15 (1), 1338-1357.

(216) Ashrafian, S.; Ataei, S. A.; Jahanshahi, M. Polym. Adv. Technol. 2016, 27 (6), 789-804.

(217) Zhang, G.; Fang, L.; Li, F.; Gao, B. RSC Adv. 2016, 6 (62), 56936-56943.

(218) Hashemi-Moghaddam, H.; Hosseni, M.; Mohammadhosseini, M. Sep. Sci. Technol. 2017, 52 (11), 1826-1834.

(219) Herrero-Hernández, E.; Rodríguez-Gonzalo, E.; Rodríguez-Cruz, M. S.; CarabiasMartínez, R.; Sánchez-Martín, M. J. Int. J. Environ. Sci. Technol. 2015, 12 (10), 30793088 .

(220) Jo, M.; Ahn, J.-Y.; Lee, J.; Lee, S.; Hong, S. W.; Yoo, J.-W.; Kang, J.; Dua, P.; Lee, D.K.; Hong, S.; Kim, S. Oligonucleotides 2011, 21 (2), 85-91. 
(221) Qiu, H.; Luo, C.; Sun, M.; Lu, F.; Fan, L.; Li, X. Anal. Chim. Acta 2012, 744, 75-81.

(222) Qi, P.; Wang, J.; Jin, J.; Su, F.; Chen, J. Talanta 2010, 81 (4-5), 1630-1635.

(223) Tarley, C. R. T.; Segatelli, M. G.; Kubota, L. T. Talanta 2006, 69 (1), 259-266.

(224) Amira Othman, N.; Fadilah Yusof, N.; Daik, R.; Shimal Mehamod, F. Int. J. Technol. 2017, 8 (1), 37.

(225) Su, L.; Guo, X.; Han, S. Anal. Methods 2014, 6 (8), 2512.

(226) Peng, H.; Wang, S.; Zhang, Z.; Xiong, H.; Li, J.; Chen, L.; Li, Y. J. Agric. Food Chem. 2012, 60 (8), 1921-1928.

(227) Ning, F.; Peng, H.; Dong, L.; Zhang, Z.; Li, J.; Chen, L.; Xiong, H. J. Agric. Food Chem. 2014, 62 (46), 11138-11145.

(228) Saad, E. M.; Madbouly, A.; Ayoub, N.; Mohamed El Nashar, R. Anal. Chim. Acta 2015, $877,80-89$.

(229) Li, X.; Tong, Y.; Jia, L.; Guan, H. Monatshefte fur Chemie 2015, 146 (3), 423-430.

(230) Asanuma, H.; Kakazu, M.; Shibata, M.; Hishiya, T.; Komiyama, M. Chem. Commun. 1997, No. 20, 1971-1972.

(231) Kim, Y. S.; Jung, H. S.; Matsuura, T.; Lee, H. Y.; Kawai, T.; Gu, M. B. Biosens. Bioelectron. 2007, 22 (11), 2525-2531.

(232) Hashim, S. N. N. S.; Schwarz, L. J.; Danylec, B.; Mitri, K.; Yang, Y.; Boysen, R. I.; Hearn, M. T. W. J. Chromatogr. A 2016, 1468, 1-9.

(233) Hashim, S. N. N. S.; Boysen, R. I.; Schwarz, L. J.; Danylec, B.; Hearn, M. T. W. J. Chromatogr. A 2014, 1359, 35-43.

(234) Zhang, Z.; Tan, W.; Hu, Y.; Li, G. J. Chromatogr. A 2011, 1218 (28), 4275-4283.

(235) Zhu, T.; Yoon, C.; Row, K. Chinese J. Chem. 2011, 29 (6), 1246-1250.

(236) Soekamto, N. H.; St Fauziah; Taba, P.; Amran, M. B. IOP Conf. Ser. Mater. Sci. Eng. 2017, 188, 12048.

(237) Puoci, F.; Cirillo, G.; Curcio, M.; Iemma, F.; Parisi, O. I.; Castiglione, M.; Picci, N. Drug Deliv. 2008, 15 (4), 253-258.

(238) Faizal, C. K. M.; Kobayashi, T. Polym. Eng. Sci. 2008, 48 (6), 1085-1093.

(239) Feng, S.; Gao, F.; Chen, Z.; Grant, E.; Kitts, D. D.; Wang, S.; Lu, X. J. Agric. Food Chem. 2013, 61 (44), 10467-10475.

(240) Nakano, H.; Morita, S.; Shigemori, H.; Hasegawa, K. Plant Growth Regul. 2006, 48, 215219. 
(241) He, J.; Liu, Y.; Fan, M.; Liu, X. J. Agric. Food Chem. 2011, 59 (5), 1582-1586.

(242) Jiao, Y.; Jia, H.; Guo, Y.; Zhang, H.; Wang, Z.; Sun, X.; Zhao, J. RSC Adv. 2016, 6 (63), 58541-58548.

(243) Wang, L.; Liu, X.; Zhang, Q.; Zhang, C.; Liu, Y.; Tu, K.; Tu, J. Biotechnol. Lett. 2012, 34 (5), 869-874.

(244) Chen, K. J.; Zheng, Y. Q.; Kong, C. H.; Zhang, S. Z.; Li, J.; Liu, X. G. J. Agric. Food Chem. 2010, 58 (24), 12710-12716.

(245) Gastal, F. J. Exp. Bot. 2002, 53 (370), 789-799.

(246) Decher, G. Science (80-. ). 1997, 277 (5330), 1232-1237.

(247) Sultan, Y.; Walsh, R.; Monreal, C.; DeRosa, M. C. Biomacromolecules 2009, 10 (5), 1149-1154.

(248) Wilson, C.; Szostak, J. W. Chem. Biol. 1998, 5 (11), 609-617.

(249) Mastronardi, E.; Tsae, P. K.; Zhang, X.; Pach, A.; Sultan, Y.; DeRosa, M. C. Methods 2016, 97, 75-87.

(250) Sultan, Y.; Derosa, M. C. Small 2011, 7 (9), 1219-1226.

(251) Malile, B.; Chen, J. I. L. J. Am. Chem. Soc. 2013, 135 (43), 16042-16045.

(252) Qin, H.; Liu, J.; Chen, C.; Wang, J.; Wang, E. Anal. Chim. Acta 2012, 712, 127-131.

(253) Chen, L.; Zeng, X.; Ferhan, A. R.; Chi, Y.; Kim, D.-H.; Chen, G. Chem. Commun. 2015, 51 (6), 1035-1038.

(254) Zhang, X.; Chabot, D.; Sultan, Y.; Monreal, C.; Derosa, M. C. ACS Appl. Mater. Interfaces 2013, 5 (12), 5500-5507.

(255) Morris, K. N.; Jensen, K. B.; Julin, C. M.; Weil, M.; Gold, L. Rna 1998, 95 (March), 2902-2907.

(256) Ruscito, A.; DeRosa, M. C. Front. Chem. 2016, 4 (May), 1-14.

(257) Hong, K. L.; Sooter, L. J. Biomed Res. Int. 2015, 2015, 1-31.

(258) Mayer, G.; Ahmed, M.-S. L. M.; Dolf, A.; Endl, E.; Knolle, P. a; Famulok, M. Nat. Protoc. 2010, 5 (12), 1993-2004.

(259) Nutiu, R.; Li, Y. Angew. Chemie - Int. Ed. 2005, 44 (7), 1061-1065.

(260) Stoltenburg, R.; Nikolaus, N.; Strehlitz, B. J. Anal. Methods Chem. 2012, 1 (1).

(261) Spiga, F. M.; Maietta, P.; Guiducci, C. ACS Comb. Sci. 2015, 17 (5), 326-333.

(262) Reinemann, C.; Freiin von Fritsch, U.; Rudolph, S.; Strehlitz, B. Biosens. Bioelectron. 
2016, 77, 1039-1047.

(263) Gold, L.; Ayers, D.; Bertino, J.; Bock, C.; Bock, A.; Brody, E. N.; Carter, J.; Dalby, A. B.; Eaton, B. E.; Fitzwater, T.; Flather, D.; Forbes, A.; Foreman, T.; Fowler, C.; Gawande, B.; Goss, M.; Gunn, M.; Gupta, S.; Halladay, D.; Heil, J.; Heilig, J.; Hicke, B.; Husar, G.; Janjic, N.; Jarvis, T.; Jennings, S.; Katilius, E.; Keeney, T. R.; Kim, N.; Koch, T. H.; Kraemer, S.; Kroiss, L.; Le, N.; Levine, D.; Lindsey, W.; Lollo, B.; Mayfield, W.; Mehan, M.; Mehler, R.; Nelson, S. K.; Nelson, M.; Nieuwlandt, D.; Nikrad, M.; Ochsner, U.; Ostroff, R. M.; Otis, M.; Parker, T.; Pietrasiewicz, S.; Resnicow, D. I.; Rohloff, J.; Sanders, G.; Sattin, S.; Schneider, D.; Singer, B.; Stanton, M.; Sterkel, A.; Stewart, A.; Stratford, S.; Vaught, J. D.; Vrkljan, M.; Walker, J. J.; Watrobka, M.; Waugh, S.; Weiss, A.; Wilcox, S. K.; Wolfson, A.; Wolk, S. K.; Zhang, C.; Zichi, D. PLoS One 2010, 5 (12).

(264) Margulies, M.; Egholm, M.; Altman, W. E.; Attiya, S.; Bader, J. S.; Bemben, L. a; Berka, J.; Braverman, M. S.; Chen, Y.-J.; Chen, Z.; Dewell, S. B.; Du, L.; Fierro, J. M.; Gomes, X. V; Godwin, B. C.; He, W.; Helgesen, S.; Ho, C. H.; Ho, C. H.; Irzyk, G. P.; Jando, S. C.; Alenquer, M. L. I.; Jarvie, T. P.; Jirage, K. B.; Kim, J.-B.; Knight, J. R.; Lanza, J. R.; Leamon, J. H.; Lefkowitz, S. M.; Lei, M.; Li, J.; Lohman, K. L.; Lu, H.; Makhijani, V. B.; McDade, K. E.; McKenna, M. P.; Myers, E. W.; Nickerson, E.; Nobile, J. R.; Plant, R.; Puc, B. P.; Ronan, M. T.; Roth, G. T.; Sarkis, G. J.; Simons, J. F.; Simpson, J. W.; Srinivasan, M.; Tartaro, K. R.; Tomasz, A.; Vogt, K. a; Volkmer, G. a; Wang, S. H.; Wang, Y.; Weiner, M. P.; Yu, P.; Begley, R. F.; Rothberg, J. M. Nature 2005, 437 (7057), $376-380$.

(265) Kupakuwana, G. V.; Crill, J. E.; McPike, M. P.; Borer, P. N. PLoS One 2011, 6 (5).

(266) Hoinka, J.; Berezhnoy, A.; Dao, P.; Sauna, Z. E.; Gilboa, E.; Przytycka, T. M. Nucleic Acids Res. 2015, 43 (12), 5699-5707.

(267) Valenzano, S.; De Girolamo, A.; DeRosa, M. C.; McKeague, M.; Schena, R.; Catucci, L.; Pascale, M. ACS Comb. Sci. 2016, 18 (6), 302-313.

(268) Illumina Inc. Illumina Sequencing Technology; San Diego, 2010.

(269) Hoinka, J.; Berezhnoy, A.; Sauna, Z. E.; Gilboa, E.; Przytycka, T. M. Res. Comput. Mol. Biol. 2014, 8394, 115-128.

(270) Alam, K. K.; Chang, J. L.; Burke, D. H. Mol. Ther. Acids 2015, 4 (August 2014), e230.

(271) Famulok, M.; Szostak, J. J. Am. Chem. Soc. 1992, 114 (c), 3990-3991.

(272) McKeague, M.; Foster, A.; Miguel, Y.; Giamberardino, A.; Verdin, C.; Chan, J. Y. S.; DeRosa, M. C. RSC $A d v$. 2013, 3 (46), 24415.

(273) Stoltenburg, R.; Reinemann, C.; Strehlitz, B. Anal. Bioanal. Chem. 2005, 383 (1), 83-91.

(274) Friedman, M. J. Agric. Food Chem. 2004, 52 (3), 385-406.

(275) Li, Y.; Geyer, C. R.; Sen, D. Biochemistry 1996, 35 (21), 6911-6922. 
(276) Velez, T. E.; Singh, J.; Xiao, Y.; Allen, E. C.; Wong, O. Y.; Chandra, M.; Kwon, S. C.; Silverman, S. K. ACS Comb. Sci. 2012, 14 (12), 680-687.

(277) Mehta, J.; Van Dorst, B.; Rouah-Martin, E.; Herrebout, W.; Scippo, M. L.; Blust, R.; Robbens, J. J. Biotechnol. 2011, 155 (4), 361-369.

(278) Yarus, M.; Widmann, J. J.; Knight, R. J. Mol. Evol. 2009, 69 (5), 406-429.

(279) Yarus, M. Life 2017, 7 (2), 13.

(280) Le, T. T.; Chumphukam, O.; Cass, A. E. G. RSC Adv. 2014, 4 (88), 47227-47233.

(281) McKeague, M.; De Girolamo, A.; Valenzano, S.; Pascale, M.; Ruscito, A.; Velu, R.; Frost, N. R.; Hill, K.; Smith, M. K.; McConnell, E. M.; DeRosa, M. C. Anal. Chem. 2015, 87 (17), 8608-8612.

(282) Jing, M.; Bowser, M. T. Anal. Chim. Acta 2011, 686 (1-2), 9-18.

(283) Niazi, J. H.; Lee, S. J.; Kim, Y. S.; Gu, M. B. Bioorg. Med. Chem. 2008, 16 (3), 12541261.

(284) Ma, X.; Wang, W.; Chen, X.; Xia, Y.; Duan, N.; Wu, S.; Wang, Z. Food Control 2015, $47,545-551$.

(285) Ma, X.; Wang, W.; Chen, X.; Xia, Y.; Wu, S.; Duan, N.; Wang, Z. Eur. Food Res. Technol. 2014, 238 (6), 919-925.

(286) Zhou, J.; Battig, M. R.; Wang, Y. Anal. Bioanal. Chem. 2010, 398 (6), 2471-2480.

(287) Frost, N. R.; McKeague, M.; Falcioni, D.; DeRosa, M. C. Analyst 2015, 140 (19), 66436651.

(288) Katilius, E.; Flores, C.; Woodbury, N. W. Nucleic Acids Res. 2007, 35 (22), 7626-7635.

(289) Cruz-Aguado, J. A.; Penner, G. J. Agric. Food Chem. 2008, 56 (22), 10456-10461.

(290) Keefe, A. D.; Cload, S. T. Curr. Opin. Chem. Biol. 2008, 12 (4), 448-456.

(291) Vater, A.; Klussmann, S. Drug Discov. Today 2015, 20 (1), 147-155.

(292) Lengfelder, W. 2bind: The new Service Provider to study Molecular Interactions molecular; 2013.

(293) Reuter, J. S.; Mathews, D. H. BMC Bioinformatics 2010, 11 (1), 129.

(294) Walsh, R.; DeRosa, M. C. Biochem. Biophys. Res. Commun. 2009, 388 (4), 732-735.

(295) Guédin, A.; Lacroix, L.; Mergny, J.-L. In Drug-DNA Interaction Protocols, Methods in Molecular Biology; 2010; Vol. 613, pp 25-35.

(296) Dieckmann, T.; Suzuki, E.; Nakamura, G. K.; Feigon, J. RNA 1996, 2 (7), 628-640. 
(297) Stoltenburg, R.; Schubert, T.; Strehlitz, B. PLoS One 2015, 10 (7), 1-23.

(298) Zheng, Y.; Wang, Y.; Yang, X. Sensors Actuators, B Chem. 2011, 156 (1), 95-99.

(299) Wei, H.; Li, B.; Li, J.; Wang, E.; Dong, S. Chem. Commun. 2007, No. 36, 3735.

(300) Tian, Y.; Wang, Y.; Sheng, Z.; Li, T.; Li, X. Anal. Biochem. 2016, 513, 87-92.

(301) Zhang, X.; Servos, M. R.; Liu, J. Langmuir 2012, 28 (8), 3896-3902.

(302) Griffin, L. C.; Tidmarsh, G. F.; Bock, L. C.; Toole, J. J.; Leung, L. L. Blood 1993, 81 (12), 3271-3276.

(303) Pietramellara, G.; Ascher, J.; Borgogni, F.; Ceccherini, M. T.; Guerri, G.; Nannipieri, P. Biol. Fertil. Soils 2009, 45 (3), 219-235.

(304) Eulberg, D.; Klussmann, S. ChemBioChem 2003, 4 (10), 979-983.

(305) Farokhzad, O. C.; Cheng, J.; Teply, B. a; Sherifi, I.; Jon, S.; Kantoff, P. W.; Richie, J. P.; Langer, R. Proc. Natl. Acad. Sci. U. S. A. 2006, 103 (16), 6315-6320.

(306) Gu, F.; Zhang, L.; Teply, B. a; Mann, N.; Wang, A.; Radovic-Moreno, A. F.; Langer, R.; Farokhzad, O. C. Proc. Natl. Acad. Sci. U. S. A. 2008, 105 (7), 2586-2591.

(307) Dhar, S.; Gu, F. X.; Langer, R.; Farokhzad, O. C.; Lippard, S. J. Proc. Natl. Acad. Sci. U. S. A. 2008, 105 (45), 17356-17361.

(308) Kang, H.; O’Donoghue, M. B.; Liu, H.; Tan, W. Chem. Commun. 2010, 46 (2), 249-251.

(309) Sercombe, L.; Veerati, T.; Moheimani, F.; Wu, S. Y.; Sood, A. K.; Hua, S. Front. Pharmacol. 2015, 6 .

(310) Anajafi, T.; Mallik, S. Ther. Deliv. 2015, 6 (4), 521-534.

(311) Dentinger, P. M.; Simmons, B. A.; Cruz, E.; Sprague, M. Langmuir 2006, 22 (7), 29352937.

(312) Pokholenko, O.; Gissot, A.; Vialet, B.; Bathany, K.; Thiéry, A.; Barthélémy, P. J. Mater. Chem. B 2013, 1 (39), 5329.

(313) Yan, Y.; Sun, Y.; Yu, H.; Xu, H.; Lu, J. R. Soft Matter 2015, 11 (9), 1748-1754.

(314) Albert, S. K.; Golla, M.; Thelu, H. V. P.; Krishnan, N.; Varghese, R. Chem. - A Eur. J. 2017, 8348-8352.

(315) Wu, Y.; Sefah, K.; Liu, H.; Wang, R.; Tan, W. Proc. Natl. Acad. Sci. 2010, 107 (1), 5-10.

(316) Wu, C.; Chen, T.; Han, D.; You, M.; Peng, L.; Cansiz, S.; Zhu, G.; Li, C.; Xiong, X.; Jimenez, E.; Yang, C. J.; Tan, W. ACS Nano 2013, 7 (7), 5724-5731.

(317) Gore, T.; Dori, Y.; Talmon, Y.; Tirrell, M.; Bianco-Peled, H. Langmuir 2001, 17 (17), $5352-5360$. 
(318) Sultan, Y. Development of Smart Materials Using Aptamer Based Bionanotechnologies, Carleton University, 2011.

(319) Sharma, A.; Sharma, U. S. Int. J. Pharm. 1997, 154 (2), 123-140.

(320) Dua, J. S.; Rana, A. C.; Bhandari, A. K. Int. J. Pharm. Stud. Res. 2012, 3 (II), 14-20.

(321) Karayianni, M.; Pispas, S. In Fluorescence Studies of Polymer Containing Systems; 2016; Vol. 16, pp 27-63.

(322) Shivalingam, A.; Izquierdo, M. A.; Marois, A. Le; Vyšniauskas, A.; Suhling, K.; Kuimova, M. K.; Vilar, R. Nat. Commun. 2015, 6, 8178.

(323) Bhasikuttan, A. C.; Mohanty, J. Chem. Commun. 2015, 51 (36), 7581-7597.

(324) Ruta, J.; Perrier, S.; Ravelet, C.; Fize, J.; Peyrin, E. Anal. Chem. 2009, 81 (17), 74687473.

(325) Mao, X.; Ma, Y.; Zhang, A.; Zhang, L.; Zeng, L.; Liu, G. Anal. Chem. 2009, 81 (4), $1660-1668$. 


\section{Appendix}

Table 7.1: Primer sequences used for Illumina high-throughput sequencing.

\begin{tabular}{|c|c|}
\hline Primer & Sequence \\
\hline F1 & $\begin{array}{l}\text { AATGATACGGCGACCACCGAGATCTACACTCTTTCCCTACACGACGCTCT } \\
\text { TCCGATCTATACCAGCTTATTCAATT }\end{array}$ \\
\hline $\mathrm{F} 2$ & $\begin{array}{l}\text { AATGATACGGCGACCACCGAGATCTACACTCTTTCCCTACACGACGCTCT } \\
\text { TCCGATCTNATACCAGCTTATTCAATT }\end{array}$ \\
\hline F3 & $\begin{array}{l}\text { AATGATACGGCGACCACCGAGATCTACACTCTTTCCCTACACGACGCTCT } \\
\text { TCCGATCTNNAGCAGCACAGAGGTCAGATG }\end{array}$ \\
\hline F4 & $\begin{array}{l}\text { AATGATACGGCGACCACCGAGATCTACACTCTTTCCCTACACGACGCTCT } \\
\text { TCCGATCTNNNAGCAGCACAGAGGTCAGATG }\end{array}$ \\
\hline R1 & $\begin{array}{l}\text { CAAGCAGAAGACGGCATACGAGATCGTGATGTGACTGGAGTTCAGACGT } \\
\text { GTGCTCTTCCGATCAGATTGCACTTACTATCT }\end{array}$ \\
\hline R2 & $\begin{array}{l}\text { CAAGCAGAAGACGGCATACGAGATACATCGGTGACTGGAGTTCAGACGT } \\
\text { GTGCTCTTCCGATCAGATTGCACTTACTATCT }\end{array}$ \\
\hline R3 & $\begin{array}{l}\text { CAAGCAGAAGACGGCATACGAGATGCCTAAGTGACTGGAGTTCAGACGT } \\
\text { GTGCTCTTCCGATCNAGATTGCACTTACTATCT }\end{array}$ \\
\hline R4 & $\begin{array}{l}\text { CAAGCAGAAGACGGCATACGAGATTGGTCAGTGACTGGAGTTCAGACGT } \\
\text { GTGCTCTTCCGATCNAGATTGCACTTACTATCT }\end{array}$ \\
\hline R5 & $\begin{array}{l}\text { CAAGCAGAAGACGGCATACGAGATCACTGTGTGACTGGAGTTCAGACGT } \\
\text { GTGCTCTTCCGATCNNTTCACGGTAGCACGCATAGG }\end{array}$ \\
\hline R6 & $\begin{array}{l}\text { CAAGCAGAAGACGGCATACGAGATATTGGCGTGACTGGAGTTCAGACGT } \\
\text { GTGCTCTTCCGATCNNTTCACGGTAGCACGCATAGG }\end{array}$ \\
\hline R7 & $\begin{array}{l}\text { CAAGCAGAAGACGGCATACGAGATGATCTGGTGACTGGAGTTCAGACGT } \\
\text { GTGCTCTTCCGATCNNNTTCACGGTAGCACGCATAGG }\end{array}$ \\
\hline R8 & $\begin{array}{l}\text { CAAGCAGAAGACGGCATACGAGATTCAAGTGTGACTGGAGTTCAGACGT } \\
\text { GTGCTCTTCCGATCNNNTTCACGGTAGCACGCATAGG }\end{array}$ \\
\hline
\end{tabular}


Table 7.2: Prevalence of DNA codons in aptamer candidate sequences.

Amino Acid

Alanine

Arginine

Asparagine

Aspartic Acid

Cysteine

Glutamic Acid

Glutamine

Glycine

Histidine

Isoleucine

Leucine

Lysine

Methionine

Phenylalanine

Proline

Serine

Threonine

Tryptophan

Tyrosine

Valine
DNA Codons

Rate of codons in aptamer

candidates

36

51

20

26

24

26

30

20

13

50

CTT, CTC, CTA, CTG, TTA, TTG 44

AAA, AAG 17

ATG 8

TTT, TTC 7

CCT, CCC, CCA, CCG 22

TCT, TCC, TCA, TCG, AGT, AGC 75

ACT, ACC, ACA, ACG 33

TGG 7

TAT, TAC 23

GTT, GTC, GTA, GTG 47 